\section{$\widehat{\overline{\Pi \| I}}$ \\ UNIVERSITEIT GENT}

Faculteit Wetenschappen

Vakgroep Vaste-stofwetenschappen

\title{
Biaxial alignment in sputter deposited thin films
}

\author{
Stijn Mahieu
}

Proefschrift voorgelegd tot het behalen van de graad Doctor in de Wetenschappen: Natuurkunde.

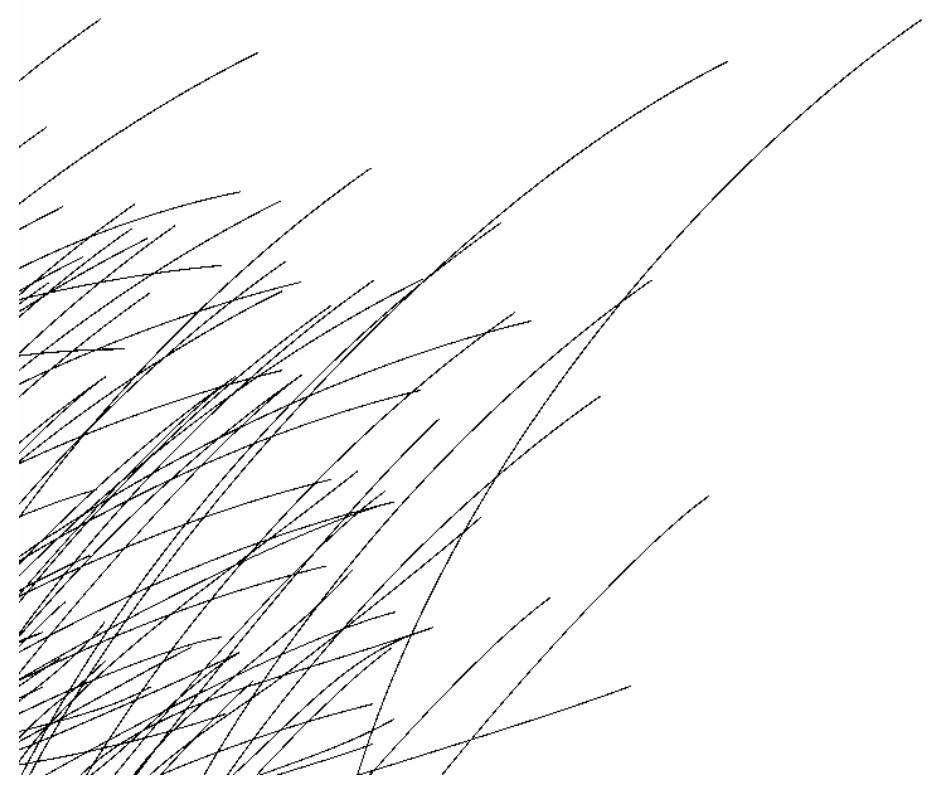
2006

Promotor: Prof. Dr. Ir. R. De Gryse Copromotor: Prof. Dr. D. Depla Voorzitter: Prof. Dr. J. Ryckebusch Juryleden:

Dr. P. Barna

Prof. Dr. C. Eisenmenger-Sittner

Dr. G. Huyberechts

Ir. A. Segers

Dr. G. Silversmit

Prof. Dr. I. Van Driessche

Prof. Dr. G. Van Tendeloo 



\section{Biaxial alignment in sputter deposited thin films: contents}

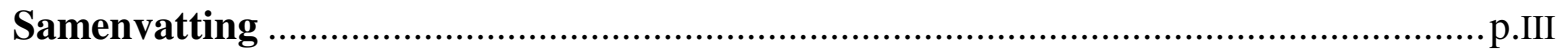

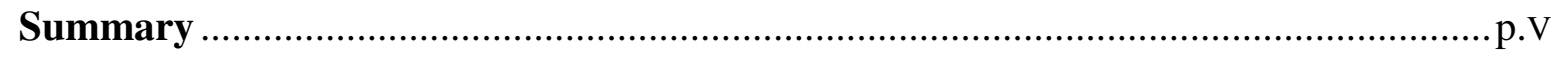

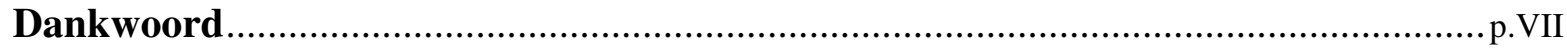

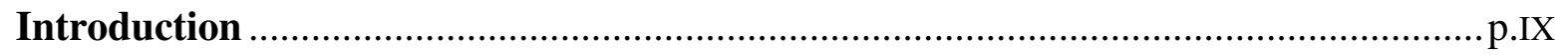

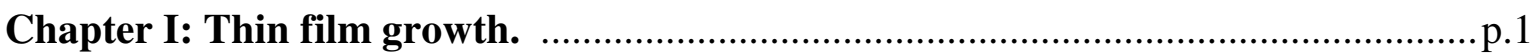

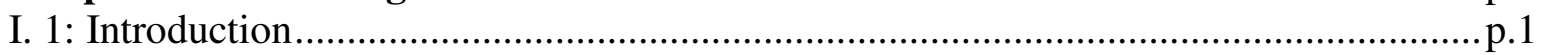

I. 2: Adsorption, desorption and diffusion ..................................................................p. 1

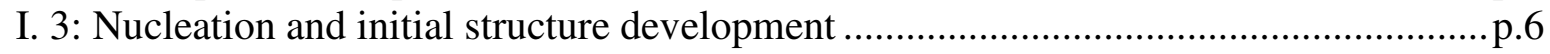

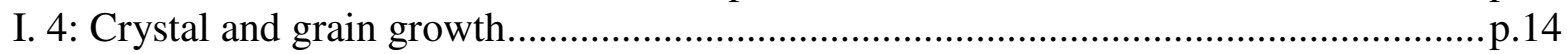

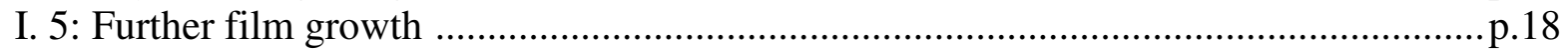

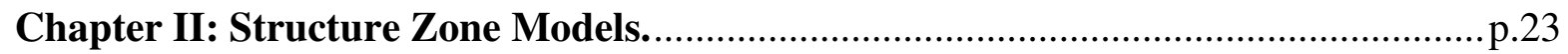

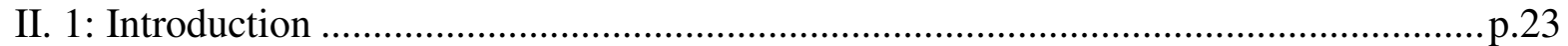

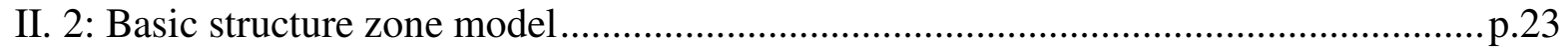

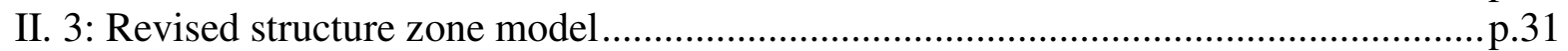

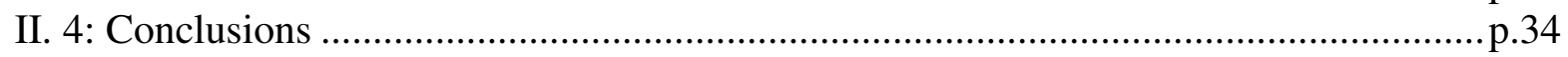

Chapter III: Reactive unbalanced magnetron sputter deposition............................p.39

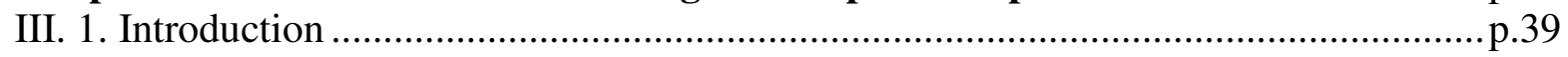

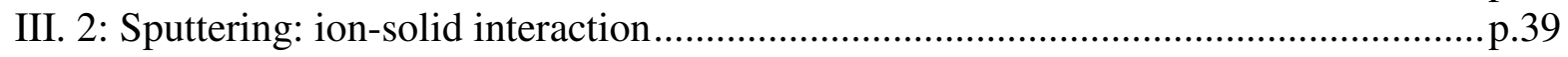

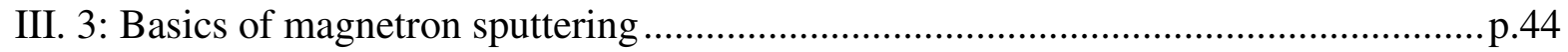

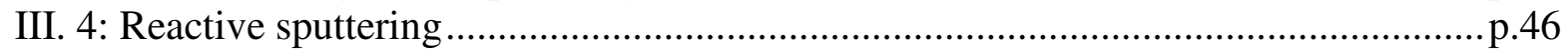

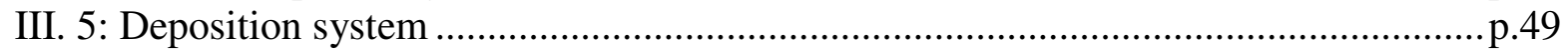

Chapter IV: Biaxially aligned films and their characterization methods.................p.53

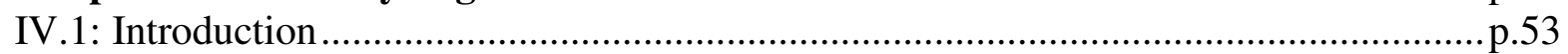

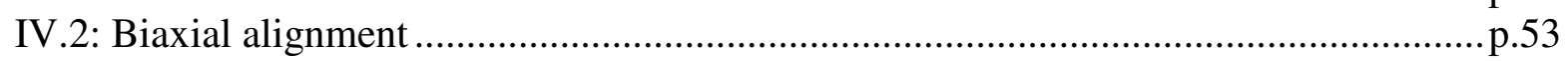

IV.3: X-ray Diffraction ................................................................................... .54

IV.4: Transmission Electron Microscopy and Diffraction ........................................... p.58

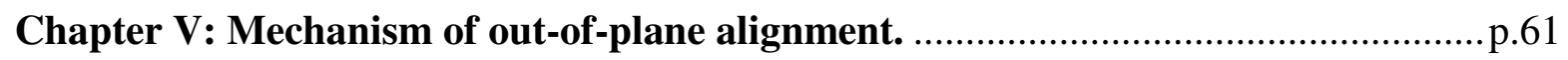

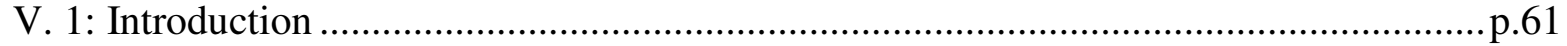

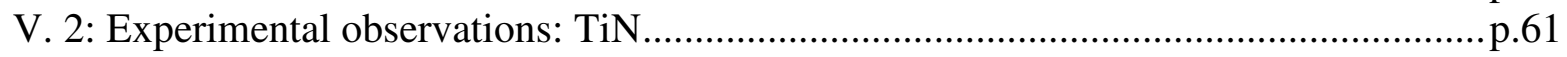

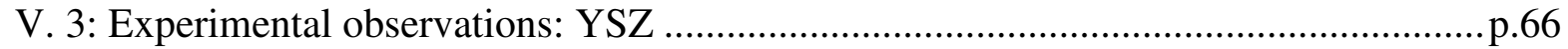

V. 4: Influence of an increasing reactive gas flow: comparison between TiN and YSZ ....p.72

V. 5: Published models for out-of-plane alignment .......................................................p. 73

V.5.1: Overall energy minimization ............................................................p. 73

V.5.2: Energetic bombardment effect............................................................ 75

V.5.3: Kinetics versus thermodynamics .......................................................p. 79

V. 6: Proposed model for out-of-plane alignment in zone T ......................................p. 84

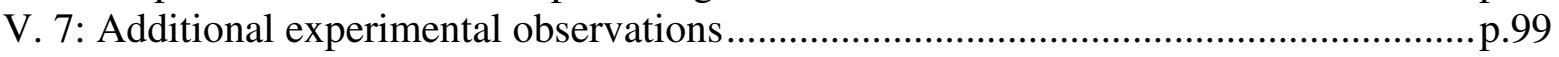

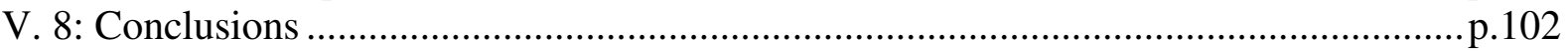




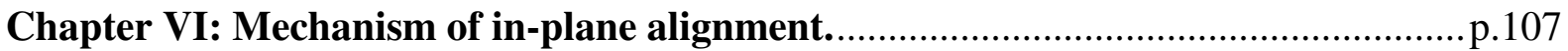

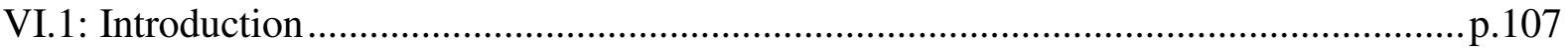

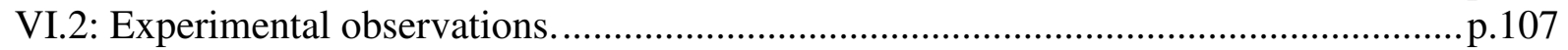

VI.3: Proposed model for the in-plane alignment .............................................................p. 112

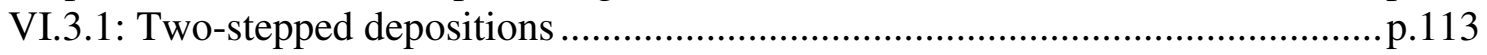

VI.3.2: In-plane growth rate anisotropy.............................................................p.118

VI.3.3: Considerations on two-stepped depositions B and D ................................p.123

VI.4: Models in literature for the in-plane alignment ..................................................

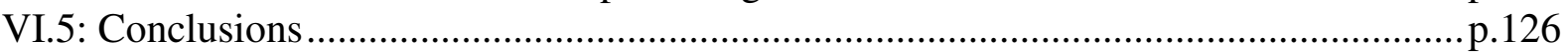

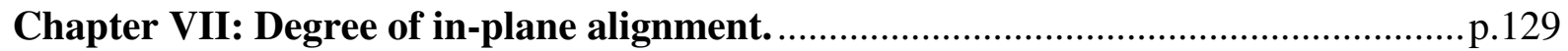

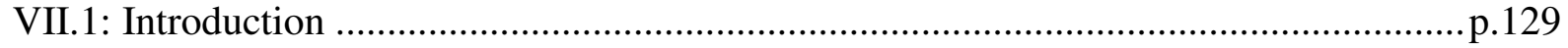

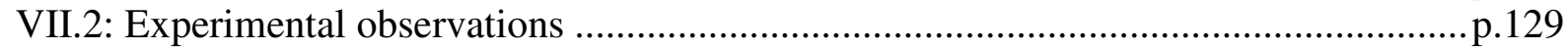

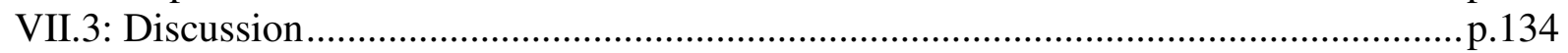

VII.4: Description of the Monte Carlo model ................................................................p.136

VII.4.1: Nascent sputter flux...........................................................................p. 136

VII.4.2: Transport through the gas phase ...........................................................p. 138

VII.4.3: Analysis of incoming material flux ....................................................... 142

VII.5: Validation of simulation code........................................................................p. 142

VII.5.1: Basic results and comparison of experimental and simulated deposition

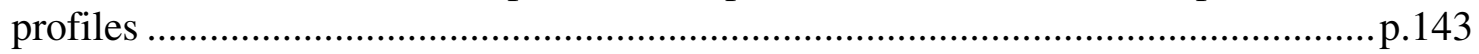

VII.5.2: Deposition profile as a function of reactive gas flow ...............................p.145

VII.6. Correlation between in-plane alignment and the deposition conditions...................p.146

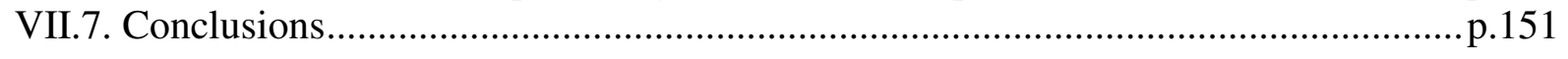

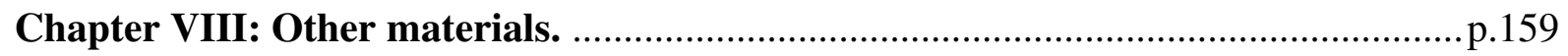

VIII.1: Introduction ............................................................................................. 159

VIII.2: Correlation between presented models and experimental observations for other

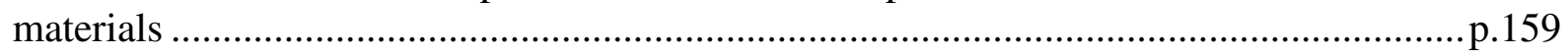

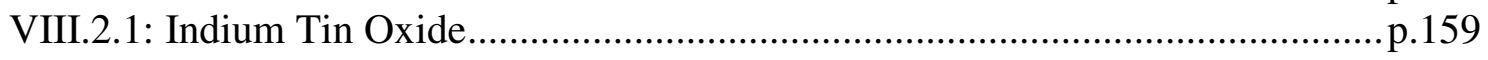

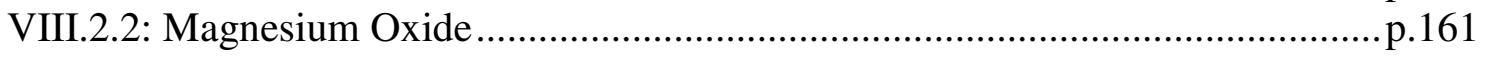

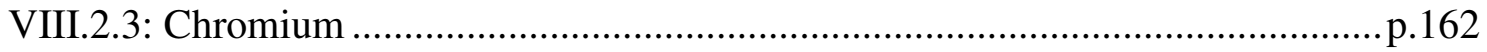

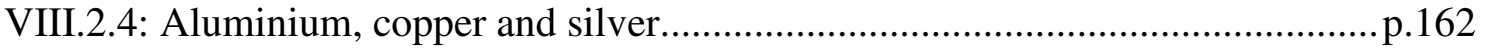

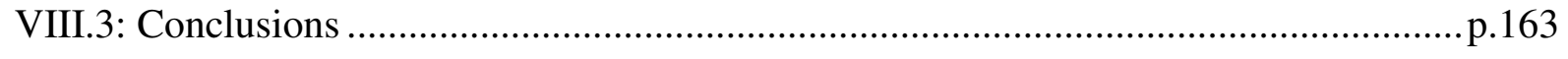

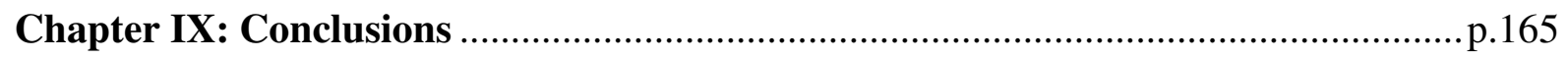

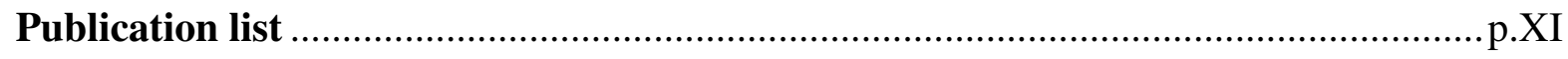




\section{Samenvatting:}

Bij industriële toepassingen worden dunne filmen steeds meer gebruikt aangezien zij op een gunstige wijze de eigenschappen van de drager, i.e. het substraat, kunnen beïnvloeden. De eigenschappen van deze deklagen zijn niet alleen materiaalafhankelijk maar worden ook mede bepaald door de omstandigheden waaronder ze werden afgezet. Immers, eigenschappen zoals hardheid, geleidbaarheid, verwerings-en corrosiebestendigheid, katalytische activiteit, optische eigenschappen of barrière-eigenschappen worden in sterke mate beïnvloed door de stochiometrie, microstructuur en de kristallografische oriëntatie van de deklaag.

Een veel gebruikte techniek om zo'n deklagen af te zetten is reactief magnetron sputteren. Dit is een plasma geassisteerde techniek waarbij op de kathode, i.e. de target, een negatieve spanning wordt aangelegd. Hierdoor worden positieve ionen naar deze kathode versneld, waar deze dan na impact op de target een botsingscascade veroorzaken. Deze botsingscascade heeft als gevolg dat sommige targetatomen zich van het oppervlak kunnen losmaken en zo na doorgang doorheen het plasma op het substraat of op de kamerwanden kunnen condenseren. Door het toevoegen van een reactief gas kan men vanaf een metallische target toch een verbinding op het substraat afzetten.

Hoewel reeds veel commerciële toepassingen gebruik maken van deklagen afgezet aan de hand van reactief magnetron sputteren, is de effectieve groei van deze deklagen nog een relatief onbekend proces. Het algemeen groeimechanisme van dunne filmen afgezet op een polykristallijn of amorf substraat aan de hand van reactief magnetron sputteren is bijgevolg een eerste onderdeel van dit onderzoek. Daarnaast werd meer specifiek onderzoek verricht naar het optreden van een biaxiale alignatie in de polykristallijne deklagen wanneer deze worden afgezet op een gekanteld substraat. Zo'n polykristallijne, biaxiaal gealigneerde deklagen zijn opgebouwd uit verschillende kristallijne korrels welke allemaal zo georiënteerd zijn dat ze niet alleen nagenoeg dezelfde kristallografische oriëntatie loodrecht op het substraatoppervlak hebben, maar eveneens een kristallografische ordening volgens een referentierichting evenwijdig aan het substraatoppervlak vertonen. Om dit algemeen groeimechanisme en het optreden van een biaxiale alignatie door substraatkanteling te onderzoeken werden zowel yttria gestabiliseerd zirconia (YSZ) als titaan nitride (TiN) deklagen afgezet op polykristallijn stainless steel en amorf glas aan de hand van reactief magnetron sputteren.

\section{Algemeen groeimechanisme:}

Gewoonlijk worden afgezette deklagen onderverdeeld in een zogenaamd structuurzone-model (SZM). Zo'n standaard SZM deelt de deklagen op in verschillende zones, i.e. zone I, T, II en III, op basis van de experimenteel vastgestelde microstructuur. In het uitgevoerde onderzoek werd aangetoond dat zo'n SZM niet alleen een nuttige onderverdeling van de afgezette deklagen weergeeft, maar onmiddellijk aantoont welke elementaire processen zoals diffusie en fundamentele groeiprocessen, i.e. nucleatie, kristalgroei en herstructurerende korrelgroei al dan niet optraden tijdens de groei. Dit alles resulteerde in het opstellen van een SZM dat de afgezette deklagen onderverdeelt in zes zones, i.e. zone Ia, Ib, Ic, T, II en III. Er werd aangetoond dat enkel in deklagen afgezet onder zone T en zone II omstandigheden zich een preferentiële uit-het-vlak alignatie ontwikkelt, i.e. dat alle korrels nagenoeg dezelfde kristallografische oriëntatie loodrecht op het substraatoppervlak hebben. Onder zone T omstandigheden ontwikkelt deze uit-het-vlak alignatie zich ten gevolge van een kinetisch beperkte groei, waarbij de korrels met de snelst groeiende oriëntatie loodrecht op het substraatoppervlak de andere korrels zullen overgroeien. Tijdens het onderzoek werd dan ook aangetoond dat de relatieve kristallografische groeisnelheid wordt bepaald door het aantal dichtste naburen aangeboden tussen de ad-deeltjes en het groeiend vlak. Het vlak dat het 
kleinste aantal dichtste naburen aanbiedt zal dan de laagste groeisnelheid hebben, en resulteren in de specifieke kristal habitus. Aangezien dit aangeboden aantal dichtste naburen afhankelijk is van de toestand van de ad-deeltjes en van de specifieke terminatie van het groeiend vlak, welke op zijn beurt dan weer afhangt van de toestand van het reactief gas, moet de exacte plasmasamenstelling gekend zijn. Deze laatste werd opgemeten aan de hand van energie geresolveerd massa-spectrometrie.

Voor zone II deposities werd beredeneerd dat de resulterende uit-het-vlak alignatie thermodynamisch bepaald is. Bijgevolg komt deze uit-het-vlak oriëntatie overeen met het vlak met de laagste oppervlakte-energie.

\section{Biaxiale alignatie:}

Experimenteel werd vastgesteld dat het uitvoeren van een hierboven beschreven zone $\mathrm{T}$ depositie op een gekanteld substraat, resulteert in een biaxiaal gealigneerde deklaag. M.a.w. werd er niet alleen een preferentiële uit-het-vlak alignatie, maar eveneens een in-het-vlak alignatie vastgesteld. Door het uitvoeren van specifieke deposities kon worden aangetoond dat deze in-het-vlak alignatie hoofdzakelijk wordt veroorzaakt door de 2D groei van de korrels, i.e. het aangroeien van de korrels door het incorporeren van ad-deeltjes die initieel op een andere plaats van het substraat of op een andere korrel landden, en dan na diffusie de groeiende korrel bereiken. Door de substraatkanteling en door het behoud van impulsmoment kan worden aangenomen dat de diffusie van deze ad-deeltjes gericht is volgens de orthogonale projectie van de invalsrichting op het substraat. Na berekening van het aantal diffunderende ad-deeltjes dat een groeiende korrel invangt, kon worden aangetoond worden dat korrels met één specifieke in-het-vlak oriëntatie de grootste groeisnelheid hebben. Bijgevolg veroorzaakt deze directionele diffusie een groeianisotropie en dus het ontwikkelen van een in-het-vlak alignatie door een overgroeiproces. Bovendien werd beredeneerd en aangetoond dat de graad van in-het-vlak alignatie een sterke correlatie vertoont met de angulaire spreiding op de invalsrichting van de materiaalflux. Door het simuleren van het sputterproces aan de target en het transport van de gesputterde deeltjes doorheen de gasfase aan de hand van een Monte Carlo methode, kon deze angulaire spreiding als functie van de depositieomstandigheden worden berekend. Bijgevolg kon een optimale in-het-vlak alignatie voor een specifieke set aan depositieomstandigheden bepaald worden.

Uiteindelijk kan besloten worden dat de resulterende microstructuur van de afgezette deklaag bepaald wordt door de totale energieflux naar het substraat tijdens de groei, aangezien deze bepaalt in welke zone de depositie wordt uitgevoerd. Indien de depositiecondities zo waren dat de afgezette deklaag werd afgezet onder zone T omstandigheden, moet ook de plasmasamenstelling gekend zijn om de uiteindelijke preferentiële uit-het-vlak alignatie te begrijpen. Wanneer dan weer een zone T deklaag wordt afgezet op een gekanteld substraat, zal naast een preferentiële uit-het-vlak alignatie ook een in-het-vlak alignatie ontstaan. Er werd aangetoond dat deze in-het-vlak alignatie een gevolg is van de anisotropie in groeisnelheid van de verschillend in-het-vlak georiënteerde korrels door enerzijds het optreden van een directionele diffusie en anderzijds het groeien van de korrels volgens hun specifieke kristal habitus. De graad van biaxiale alignatie wordt dan weer in sterke mate bepaald door de angulaire spreiding op de inkomende materiaalflux, welke berekend kon worden aan de hand van Monte Carlo simulaties.

Om de groei van een biaxiaal gealigneerde deklaag volledig te begrijpen moet men bijgevolg de totale energieflux berekenen, de plasmasamenstelling opmeten en de angulaire spreiding op de materiaalflux simuleren. De vooropgestelde groeimodellen blijken eveneens geldig te zijn voor $\mathrm{Cr}, \mathrm{MgO}$ en Indium Tin Oxide. 


\section{Summary:}

Thin films still gain interest for industrial applications since they influence the substrate properties in a positive way. The properties of these coatings are not only material dependent, but are also influenced by the deposition process itself. Indeed, properties like hardness, conductivity, corrosion resistance, catalytic activity, optical or barrier properties strongly depend on the thin film stochiometry, microstructure and crystallographic orientation. An often used technique to deposit such thin films is reactive magnetron sputtering. This is a plasma assisted technique in which a negative bias is applied to the cathode, i.e. the target. This causes the acceleration of positive ions towards the cathode or target, where these ions after impact- give rise to a collision cascade. This collision cascade results in the ejection of some surface atoms of the target, which will condense on the substrate or the chamber walls after transfer through the gas phase. By adding a reactive gas to this discharge, a compound can be deposited on the substrate using a pure metallic target.

Although already a lot of commercial applications make use of reactive sputter deposited thin films, the exact growth process of these films is still relative unknown. Therefore, the general growth mechanism of reactive sputter deposited thin films is a first topic of this research.

Next to it, more specific research was carried out to the development of a biaxial alignment in polycrystalline thin films deposited on a tilted substrate. Such polycrystalline, biaxial aligned thin films consist of different crystalline grains which are all oriented in that way that they not only have nearly the same crystallographic orientation perpendicular to the substrate, but also exhibit a crystallographic alignment along a reference direction parallel to the substrate plane. Yttria stabilized zirconia (YSZ) and titanium nitride (TiN) thin films were deposited on polycrystalline stainless steel and amorphous glass by reactive magnetron sputtering to investigate this general growth mechanism and the development of the biaxial alignment.

General growth mechanism:

Deposited thin films are usually catalogued into a so-called structure zone model (SZM). Such a standard SZM subdivides the thin films in different zones, i.e. zone I, T, II and III, based on the experimentally observed microstructure. It has been shown in the performed research that such a SZM is not only a useful subdivision of the deposited films, but also immediately gives information about which elementary processes like diffusion and fundamental phenomena of structure formation, i.e. nucleation, island growth and restructurative grain growth occurred during the growth. This resulted in the development of a new SZM subdivided into six different zones, i.e. zone Ia, Ib, Ic, T, II and III.

It was shown that only the thin films deposited in zone $\mathrm{T}$ and zone II conditions result in a deposition process related preferential out-of-plane orientation, i.e. that all grains share a crystallographic orientation perpendicular to the substrate.

In zone T conditions, this out-of-plane alignment is a result of a kinetically limited growth in which the grains oriented with the fastest growing direction perpendicular to the substrate overgrow all other grains. During the research, it has been shown that the relative crystallographic growth rate depends on the number of nearest neighbours offered between the incoming ad-particles and the growing plane. The plane that offers the smallest amount of nearest neighbours will have the smallest perpendicular growth rate, leading to the specific crystal habit. Since that number of nearest neighbours depends on the state of the ad-particles and on the exact termination of the growing plane, which depends on the state of the reactive gas, the plasma composition should be known. This composition has been measured by energy-resolved mass spectrometry. 
In zone II conditions, the out-of-plane alignment was reasoned to be thermodynamically driven. This means that the resulting out-of-plane orientation corresponds to the crystallographic plane of lowest surface energy.

\section{Biaxial alignment:}

Performing a deposition in the earlier described zone $\mathrm{T}$ conditions on a tilted substrate, a biaxial aligned thin film was observed. Hence, not only a preferential out-of-plane orientation, but also a crystallographic in-plane alignment resulted. By carrying out specific depositions, it could be shown that this in-plane alignment is mainly caused by the $2 \mathrm{D}$ growth of the grains, i.e. the growth due to the incorporation of ad-particles which initially stroke on the bare substrate or another grain and then diffused towards the expanding grain. Due to the substrate tilt and the conservation of momentum, this diffusion of the ad-particles will be directed along the orthogonal projection of the incoming flux on the substrate plane. By calculating the amount of diffusing ad-particles captured by the grains, it could be shown the grains with a specific in-plane orientation will have the largest growth rate. Hence, this directional diffusion causes an in-plane growth rate anisotropy and thus the development of an in-plane alignment by an overgrowth mechanism. Moreover, it has been shown that the degree of in-plane alignment is strongly correlated to the angular spread of the incoming material flux. By simulating the nascent sputter flux and the collisional transport through the gas phase of these sputtered particles by a binary collision Monte Carlo method, that angular spread was calculated as a function of the deposition conditions. Consequently, an optimal in-plane alignment for a specific set of deposition conditions could be determined.

Finally, it can be concluded that the resulting microstructure of the deposited films is determined by the total energy flux towards the substrate during growth, since that energy flux determines in which zone the deposition is carried out. If the deposition is carried out in zone T conditions, also the plasma composition should be known to fully understand the resulting out-of-plane alignment. Carrying out such a zone $\mathrm{T}$ deposition on a tilted substrate not only results in a preferential out-of-plane oriented film, but also in a crystallographic inplane alignment. This in-plane alignment was shown to be a result of an anisotropic growth rate of the different in-plane oriented grains due to the directional diffusion on the one hand and due to the growth of the grains according their specific crystal habit on the other hand. The degree of in-plane alignment is mainly determined by the angular spread of the incoming material flux which could be calculated by Monte Carlo simulations.

As a conclusion, a complete knowledge of the growth of biaxial aligned thin films needs the calculation of the energy flux towards the substrate, the characterization of the plasma composition and the simulation of the angular spread of the material flux. The proposed growth models are also in agreement with the experimental results of sputtered $\mathrm{Cr}, \mathrm{MgO}$ and Indium Tin Oxide thin films. 


\section{Dankwoord:}

Vooraleer de resultaten van mijn onderzoek te bespreken zou ik willen benadrukken dat deze er niet zouden gekomen zijn zonder de steun van vele anderen.

Vooreerst wil ik het Bijzonder Onderzoeksfond UGent (BOF) en het Instituut voor de aanmoediging van Innovatie door Wetenschap en Technologie in Vlaanderen (IWT-

Vlaanderen) danken voor de financiële steun! De firma Bekaert bewees me een grote dienst door het uitlenen van hun massa-spectrometer. Daarnaast wil ik ook in het bijzonder mijn promotoren, Roger en Diederik, bedanken om mij in de sputtergilde te introduceren en dus kennis te laten maken met deze mooie techniek! Pieter en Sarah wil ik bedanken om de vele discussies die we als collega's voerden omtrent biaxiale alignatie of ander sputter gerelateerde zaken... Ook Olivier hielp me steeds vol enthousiasme bij de SEM en XRD metingen. Dank aan Gilbert voor de vele "inox-stukken" die hij de afgelopen jaren voor me maakte en natuurlijk ook aan alle andere S1 collega's wie ik ooit hulp vroeg. Zonder Oleg Lebedev en Staf Van Tendeloo zouden dan weer de mooie TEM resultaten er niet gekomen zijn. Speciaal zou ik nogmaals Diederik en Nadine willen bedanken voor de meer dan noodzakelijke ondersteuning die ik van hen kreeg tijdens de eerder moeilijke inburgerperiode... Natuurlijk ook nog een speciaal woordje van dank aan Jo, op wie ik steevast van vroeg in de ochtend kon rekenen... Also thanks to many authors who send their preprints to me, in special to Peter Barna who delivered me the most unfindable articles. Also thanks for the nice and living discussions, Peter.

I also gratefully acknowledge all members of the jury for reading my thesis.

Naast al dat sputter gedoe -hoe mooi het ook kan zijn- is er natuurlijk nog een veel mooiere wereld: die van familie en vrienden! Daarom wil in de eerste plaats mijn ouders en zussen bedanken voor de steun (en het begrip) bij mijn keuze om wis-en natuurkunde te studeren. Hierbij wil ik dan ook mijn vroegere wiskundelerares Doris bedanken om me te overtuigen natuurkunde te studeren, in plaats van het oorspronkelijk idee: wiskunde.

Natuurlijk wil ik ook mijn vrouw, Katleen, bedanken voor alle steun en hulp gedurende de afgelopen jaren! Zeker tijdens de laatste maanden, waarin ik waarschijnlijk meer aan dat sputteren dacht dan aan jou: ik beloof beterschap!

Natuurlijk wens ik ook al die $\mathrm{Zr}, \mathrm{Y}, \mathrm{Ti}, \mathrm{N}$ en $\mathrm{O}$ atomen te danken die deelnamen aan de groei zoals ik het hen vroeg... Anders kwamen die resultaten nooit zo mooi overeen met de vooropgestelde modellen! 


\section{Introduction:}

Although thin films are already frequently used in commercial applications since they influence the substrate properties in a positive way, fundamental research to that thin film deposition should go on. Often, these films are deposited by physical vapour deposition (PVD) techniques, of which reactive magnetron sputtering is an important one.

In this research, the growth of biaxially aligned thin films, i.e. of about $1 \mu \mathrm{m}$, deposited by reactive magnetron sputtering has been investigated. Such biaxially aligned thin films are polycrystalline films in which the different grains not only share a crystallographic orientation perpendicular to the substrate, but also have an alignment along a reference direction parallel to the substrate. Hence, optimizing that biaxial alignment drives the film to be "monocrystalline"-like.

During earlier research in our group, a reactive unbalanced magnetron sputtering based technique has been developed to deposit such biaxially aligned thin films. This technique was developed to deposit appropriate buffer layers for high temperature superconducting top layers. Such buffer layers not only need the right chemical and thermal properties but should also be biaxially aligned to solve the weak-link behaviour of superconducting layers, i.e. a correlation between the maximum current and the biaxial alignment. Hence, these superconducting top layers has to be grown epitaxially on already as good as possible biaxially aligned buffer layers. Since improving the degree of biaxial alignment by a trial and error method seemed inefficient, the mechanism responsible of the alignment should be investigated.

Beside to possible use as buffer layers for superconducting top layers, such biaxially aligned thin films were also shown to improve the properties of (hard) magnetic metallic films, piezoelectric materials and highly oriented diamond where the highly oriented nature of the crystallites was shown to enhance its thermal transport characteristics over that of randomly oriented diamond.

However, before focussing on that biaxial alignment, the thin film growth in general should be understood. An introduction to that general growth mechanism has been described in chapter I, in which it becomes clear that the resulting characteristics of the thin film, i.e. the microstructure and crystallographic orientation, strongly depend on the elementary processes and fundamental phenomena of structure formation and thus on the used deposition conditions. These elementary processes are e.g. substrate diffusion, self-surface diffusion and bulk diffusion while the fundamental phenomena of structure formation are nucleation, crystal growth and re-structurative grain growth. Chapter II summarizes that influence of these processes and phenomena on the resulting microstructure and crystallographic orientation, giving rise to a structure zone model (SZM).

Since reactive magnetron sputtering is an already well known technique in our research groups and since its is also an industrial up scalable technique and it was used as deposition method for all investigated thin films, the basics of this technique has been described in chapter III. A next chapter discusses in more detail the specific characteristics of a biaxial alignment.

Because a biaxial alignment can't be obtained without having a preferential out-of-plane orientation, i.e. the share of a crystallographic orientation perpendicular to the substrate of all grains, chapter $\mathrm{V}$ describes the development of that out-of-plane orientation. Beside a description of the experimental observations, a model for the out-of-plane alignment is proposed. That model is in close correlation with the SZM discussed in chapter II. Once the preferential out-of-plane orientation was obtained, also an in-plane alignment could be introduced by tilting the substrate with respect to the material flux, at least at specific 
deposition conditions. The general mechanism responsible of that in-plane alignment is described in chapter VI, resulting in a model for the development of in-plane alignment. The observed influence of several deposition parameters on the degree of in-plane alignment will be described in chapter VII. Making use of the proposed model and the simulation of the metal flux towards the growing film by a binary collision Monte Carlo method, the influence of most of these deposition parameters on the degree of in-plane alignment was understood. Although the experimental work and the proposed models are focused on yttria stabilized zirconia (YSZ) and titanium nitride (TiN), also the growth and biaxial alignment of other materials, e.g. $\mathrm{MgO}, \mathrm{Cr}$ and Indium Tin Oxide seems to be understood, as described in chapter VIII.

Although not all questions are answered (on the contrary), I hope that this thesis will bring some more insight into the growth mechanism of biaxially aligned thin films.

Enjoy the reading!

Stijn 


\section{Chapter I: Thin film growth.}

\section{1: Introduction}

Although it is not the main goal of this work to investigate all atomistic processes during the growth of a thin film, one should have enough knowledge about these processes allowing to investigate the mechanism of biaxial alignment in thin films.

Therefore, the processes of film growth and the underlying principles which determine the structure and morphology of the film are described in more detail.

This film growth may be considered as a sequence of substeps which are schematically given in figure I.1.1. and which will be described one by one in this chapter.

Depositing a film by physical vapor deposition (PVD) implies that particles will hit a bare substrate, after which these particles can impinge, adsorb, diffuse or desorb on it (§ I.2).

These condensed particles may eventually stick together and form a stable nucleus (§I.3), after which these nuclei will further expand (crystal growth) and a film will develop.

Depending on the used conditions, three different growth modes are possible: Volmer-Weber, Stranski-Krastanov and Frank-van der Merwe growth. In case of Volmer-Weber growth, the nuclei become islands, which will grow and finally touch each other. This touching or contact between neighbouring islands is from now on called impingement. Before, during and after impingement these islands possibly coalesce, restructure or consume each other. These processes are called re-structurative grain growth (§I. 4). Continuing the deposition invokes a further film growth and the determination of its final structure and morphology (§ I.5).

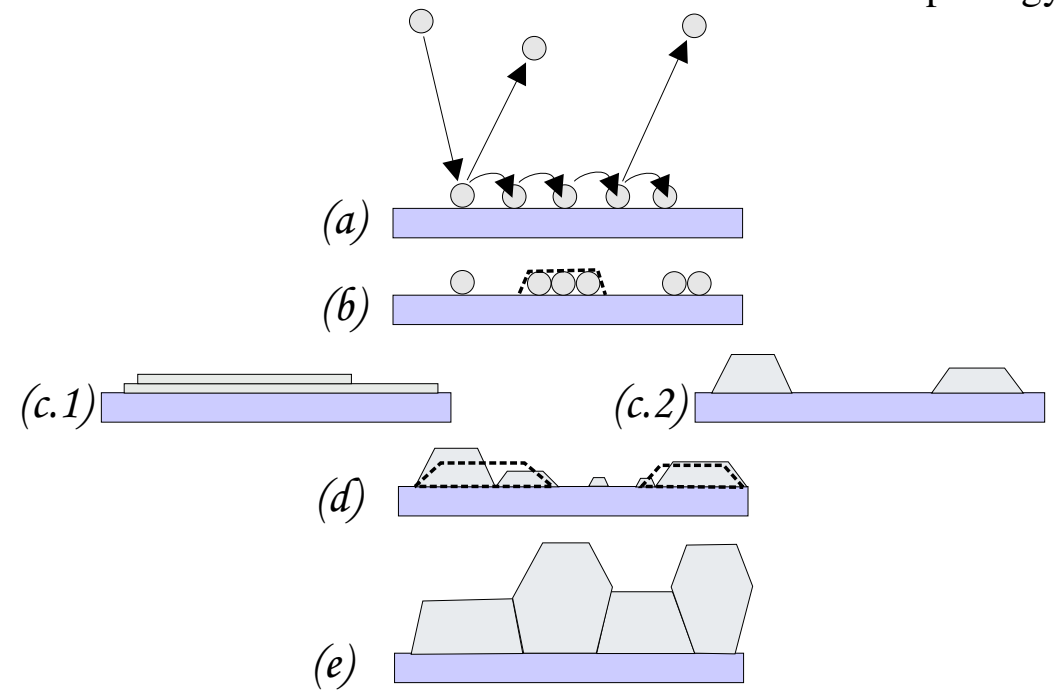

Figure I.1.1: Scheme of several stages during the thin film growth: adsorption, diffusion and desorption (a); nucleation (b); crystal growth and initial film formation (c); re-structurative grain growth (d) and further film growth (e).

\section{2: Adsorption, desorption and diffusion}

During the deposition, the bare substrate is exposed to the vapor phase and several particles will impinge on it, after which they may immediately re-evaporate or adsorb and diffuse along the surface. Gaining energy in the tail of the thermal energy distribution, they may eventually obtain enough energy to desorb again. These processes are schematically represented in figure I.2.1. In all these processes, characteristic energy barriers have to be overcome. It means that the number of particles $v$ ' being able to participate in a particular process is given by an 
Arrhenius-type exponential law: $v^{\prime} \propto \exp \left(-E_{a} / k T\right)$, with $E_{a}$ the activation energy for that particular process.

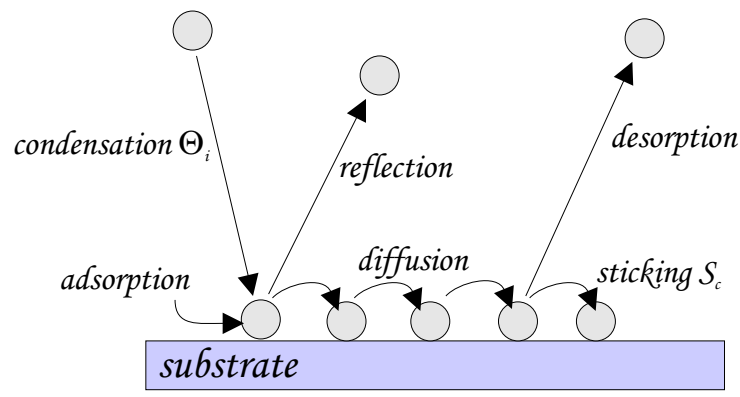

Figure I.2.1: Schematic diagram of processes such as adsorption, diffusion and desorption on the surface during the initial growth stadium.

The flux of impinging particles on the substrate is expressed by $\Theta_{i}$. When the flux is originating from an ideal gas, this flux is expressed by $\Theta_{i}=p /(2 \pi M k T)^{1 / 2}$ where $p$ is the partial pressure, $M$ the molecular weight of the particles, $k$ the Boltzmann constant and $T$ the temperature of the gas. The sticking coefficient $S_{c}$ is defined as the fraction of the impinging particles which remains adsorbed and becomes incorporated in the final film.

\section{Adsorption:}

A particle from the vapor phase approaching a surface within a few atomic distances will feel an attraction due to the interaction with the surface molecules. This interaction is known as the van der Waals force and is discussed extensively in [1,2]. This adsorption process is called physisorption and is characterized by stretching or bending the particle, but retaining its chemical identity. The particle is attracted into a potential well as shown in figure I.2.2. When moving towards the surface (lowering the potential energy), it is passing a minimum and is then repelled by a steeply increasing potential energy. The depth of the potential well is the binding energy of a physisorbed particle $E_{\text {phys }}$ and is in the order of 0.01 à $0.25 \mathrm{eV}[2,3]$. The equilibrium spacing between the physisorbed particle and the surface is in the order of $3-10 \AA$ [2].

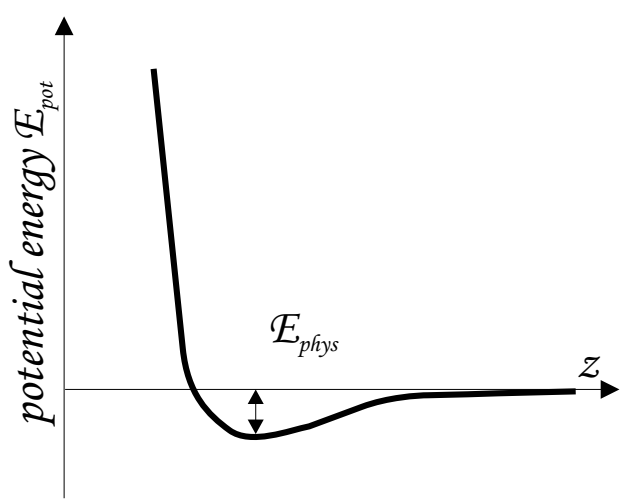

Figure I.2.2: Potential energy curve as a function of the distance to the surface $\mathrm{z}$ of the physisorbed particle.

Due to the low binding energy $E_{\text {phys }}$, the physisorbed particle is mobile and will be able to diffuse around. Sometimes, the particles are able to become stronger bonded, i.e. chemisorption. This implies the formation of a chemical bond or the sharing of electrons in new orbitals $[1,2,4,5]$. In case of chemisorption, the shape of the potential energy curve will depend on whether the incoming particle (molecule or atom) is adsorbed intact, without breaking any intermolecular bonds, or whether the molecule must dissociate to reach the chemisorbed state. These different potential curves are shown in figure I.2.3. 


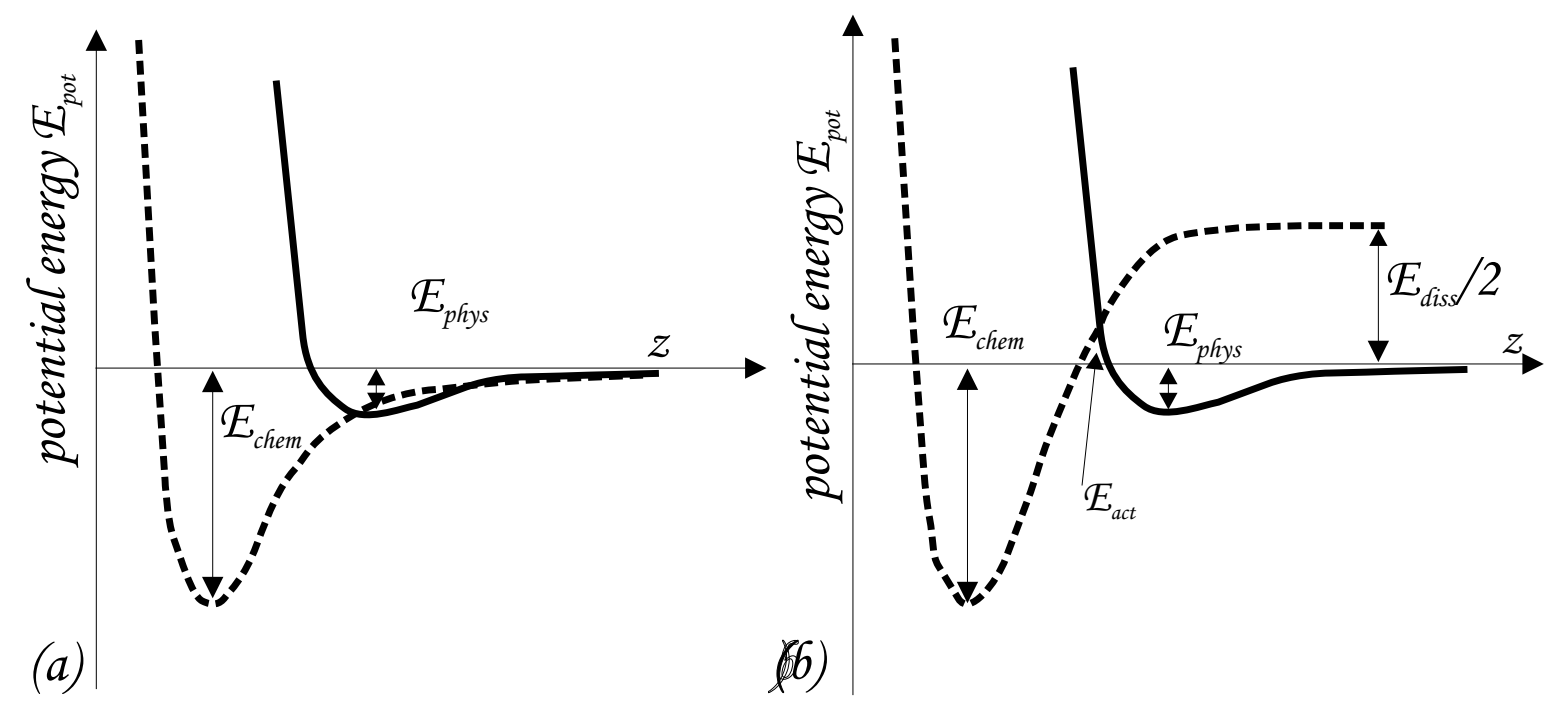

Figure I.2.3: Potential energy curves as a function of the distance $\mathrm{z}$ to the surface of the chemisorbed particle in case of non-dissociative chemisorption (a) and activated dissociative chemisorption of a homonuclear diatomic molecule (b). The full lines represent the physisorption and the dashed lines the chemisorption process.

On the one hand, in case of non-dissociative chemisorption, a particle lowers its potential energy by performing a chemical bond with the surface. Non-dissociative chemisorption happens for an impinging atomic particle, but e.g. also for a $\mathrm{C} \equiv \mathrm{O}$ molecule which gets bond at the surface without breaking its $\mathrm{C} \equiv \mathrm{O}$ bond.

On the other hand, a molecule can also chemisorb on the surface while breaking its intermolecular bonds, which is called dissociative chemisorption. In this case, mostly a crossover between the curves for the physisorbed and chemisorbed states occurs at an energy larger than zero (see fig. I.2.3.b). The chemisorption process is called to be activated by the physisorption, with a finite activation energy $E_{a c t}$ being required to surmount the energy barrier leading to the chemisorbed state. In the vapor phase, the difference in potential energy between a homonuclear diatomic molecule and a dissociated atomic part of it, is half of the dissociation energy $E_{\text {diss }}$.

The chemisorbed particle is bond with an energy $E_{\text {chem }}$, which is typically in the order of 1-10 $\mathrm{eV}$ [3], while the distance between the chemisorbed particle and the substrate is in the order of 1 à $3 \AA[0]$.

From figure I.2.3.b it is evident that chemisorption of a homonuclear diatomic molecule into its atomic adsorption state requires a minimum kinetic energy $E_{a c t}$. Since this activation barrier is lower than the dissociation energy $E_{d i s s}$ in the vapor phase, dissociation is favored by adsorption on the surface. The decrease of the activation barrier by the presence of a solid surface for dissociation is a feature of catalytic decomposition. Some values of this activation barrier are given in table I.2.4.

There are two important ways in which particles can arrive at the surface having an energy larger than zero, and hence enhancing the dissociative chemisorption. On the one hand, molecules can already be dissociated in the vapor phase (e.g. due to plasma interaction). This means that these dissociated particles arrive along the dashed curve in fig. I.2.3.b, above the activation barrier $E_{a c t}$, and hence chemisorb directly as atoms. On the other hand, sputtered particles have an energy of several eV (see chapter III and VII) and can also easily overcome the activation barrier. 
As outlined in the introduction, adsorbed particles possibly desorb from the surface if they obtain enough energy to overcome the desorption barrier $E_{d e s}$. In case of physisorption, the desorption barrier $E_{d e s}$ is equal to $E_{\text {phys }}$ and thus rather low. However, in case of chemisorption, it can be visualized in figure I.2.3.b. that the desorption barrier $E_{d e s}$ is equal to $E_{c h e m}+E_{a c t}$. As such, if the deposition is carried out on a surface at room temperature, it can be assumed that the desorption rate from the chemisorbed state is nearly zero.

Table I.2.4: Some values from literature of the activation energy $E_{\text {act }}$ for dissociative chemisorption of an $\mathrm{O}_{2}$ molecule on several metallic surfaces. The dissociation energy of an $\mathrm{O}_{2}$ molecule is $5.16 \mathrm{eV}$.

\begin{tabular}{|l|l|l|}
\hline Metal surface & $\mathrm{E}_{\text {act }}(\mathrm{eV})$ & reference \\
\hline $\mathrm{Pt}(100)$ & 0.7 & 6 \\
\hline $\mathrm{Pt}(111)$ & 0.35 à 0.4 & 7 and 8 \\
\hline $\mathrm{W}(110)$ & 0.3 & 9 \\
\hline $\mathrm{Al}(111)$ & 0.3 & 10 \\
\hline $\mathrm{Ag}(110)$ & 0.26 à 0.28 & 11 \\
\hline $\mathrm{Ag}(111)$ & 0.73 & 12 \\
\hline $\mathrm{Cu}(110)$ & 0.35 & 13 and 14 \\
\hline
\end{tabular}

\section{Diffusion:}

Physisorption or chemisorption both implies that the particle is bond to the surface. It has been discussed that the particle resides into a potential well with depth of $E_{\text {phys }}$ or $E_{\text {chem }}$. However, the former discussion ignores that this well depth $E_{\text {phys }}$ or $E_{\text {chem }}$ can vary with the position, $x$, along the surface. This variation along a pristine surface is shown in figure I.2.5.
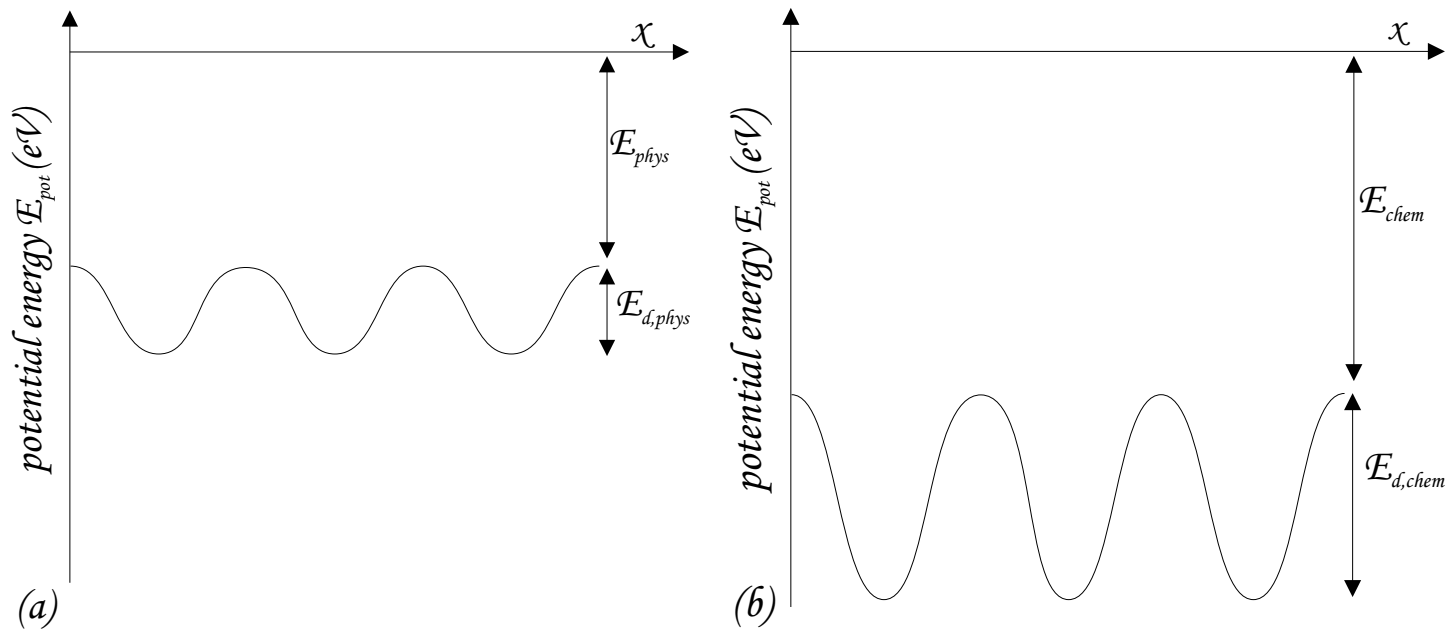

Figure I.2.5: Variation of the potential energy along the surface for a physisorbed (a) and chemisorbed (b) particle.

When an adsorbed particle attempts to change its position along the surface (diffusion), it has not to overcome the binding energy $E_{\text {phys }}$ or $E_{\text {chem }}$, but only the variation in potential energy along the surface $\left(E_{d, p h y s}\right.$ or $\left.E_{d, c h e m}\right)$. This diffusion barrier for a physisorbed particle $E_{d, \text { phys }}$ is typically in the order of $0.1 \mathrm{eV}$ [5] while the diffusion barrier for a chemisorbed particle $E_{d, \text { chem }}$ is in the order of $0.3 \mathrm{à} 2 \mathrm{eV}$ [5]. These diffusion barriers are smaller than the binding energy, because diffusion only requires rotation and elongation of bonds and no breaking of them (see figure I.2.6). This difference between the binding energy $E_{c h e m}$ and the diffusion barrier $E_{d, c h e m}$ for a chemisorbed Ti adatom on a TiN[100] and TiN[111] surface has been calculated by Gall et al.[15] and is given in table I.2.7. 


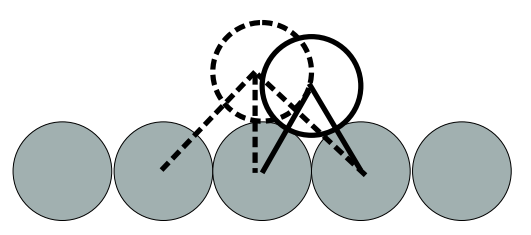

Figure I.2.6: Schematic drawing of the diffusion process of an adatom on a pristine surface. No bonds have to be broken, only elongated and rotated. The dashed lines represent an adatom in its transition state between two potential wells, while the full lines represent an adatom in the potential well.

Table I.2.7: Binding energy and diffusion barrier of a chemisorbed Ti adatom on a TiN surface as calculated by Gall et al. [15].

\begin{tabular}{|l|l|l|}
\hline surface & $E_{\text {chem }}(\mathrm{eV})$ & $E_{d, \text { chem }}(\mathrm{eV})$ \\
\hline TiN[100] & 3.3 & 0.35 \\
\hline TiN[111] & 10.09 & 1.74 \\
\hline
\end{tabular}

The diffusion rate $D$ can be expressed by $D=D_{0} e^{-E_{d} / k T}$ in which $E_{d}$ is the diffusion barrier ( $E_{d, p h y s}$ or $\left.E_{d, c h e m}\right), k$ the Boltzmann constant and $T$ the surface temperature. $D_{0}$ is the preexponential factor and typical values of it are in the order of $10^{-3} \mathrm{~cm}^{2} / \mathrm{s}[16]$. Assuming that the diffusion process can be approximated by a random walk (no net motion in any direction), the diffusing particles will be dispersed in a Gaussian distribution whose median is the starting point. Consequently, the diffusion length $\Lambda$ can be approximated by the standard deviation of this Gaussian distribution $\sigma$, which is the square root of the diffusion rate $D$ multiplied by the diffusion time $t: \Lambda=\sigma=\sqrt{D t}$.

According to Liu et al. [16], the pre-exponential factor $D_{0}$ can be written as:

$$
D_{0}=\frac{n v l^{2}}{2 \alpha} \text { (eq. I.2.1) }
$$

in which $v$ is the attempt frequency to cross the diffusion barrier, $l$ the jump length to an adjacent site (potential well), $\alpha$ the dimensionality of the space ( $\alpha=2$ for surface diffusion) and $n$ is the number of jump directions available to the adatom (e.g. 4 in case of diffusion on a fcc(100) plane). It was also discussed by Liu et al. that the attempt frequency $v$ can be computed from

$$
v=\frac{1}{2 \pi} \sqrt{\frac{c}{m}} \text { (eq.I.2.2) }
$$

in which $c$ is a force constant related to the curve of the potential energy and $m$ is the mass of the diffusing particle.

Assuming typical values $\left(\nu=10^{13} \mathrm{~s}^{-1}[5,15], E_{d, \text { chem }}=1.4 \mathrm{eV}, E_{d, p h y s}=0.1 \mathrm{eV}, T=500 \mathrm{~K}, n=\right.$ $4, l=0.3 \mathrm{~nm})$, the diffusion length of an adatom on a fcc(100) plane during 1 second can be calculated. A physisorbed adatom has a diffusion length of $285 \mu \mathrm{m}$, while a chemisorbed adatom has a diffusion length of only $0.05 \mathrm{~nm}$. Hence, it can be assumed that the final location of an adatom on a surface is determined as soon as it gets chemisorbed, unless the surface temperature is very high.

\section{Thin film growth:}

Above, it has been discussed which processes may happen when a particle impinges on a surface. It may be reflected or adsorbed. When it is physisorbed, the particle can have a rather large diffusion rate due to the low diffusion barrier $E_{d, p h y s}$. Such a diffusing physisorbed particle may also desorb again, by gaining energy in the tail of the energy distribution. The desorption rate from this physisorbed state can be high, especially at higher surface 
temperatures, due to the low desorption barrier $E_{\text {des }}\left(=E_{\text {phys }}\right)$. However, the diffusing physisorbed particle may also get chemisorbed. At moderate surface temperatures, it can be assumed that such a chemisorbed particle will stick at this position to the surface due to the rather high diffusion barrier $\left(E_{d, c h e m}\right)$ and the high desorption barrier $E_{d e s}\left(=E_{c h e m}+E_{a c t}\right)$. However, when carrying out depositions on a high temperature surface, the desorption rate from the chemisorbed state should also be taken into account.

Also the influence of the condensing flux should be taken into account to have an idea of the diffusion length $\Lambda$ and sticking coefficient $S_{c}$ (which is the fraction of the impinging particles which remains adsorbed and becomes incorporated in the final film). This because a diffusing particle may be buried by the next deposited particles before it can desorb. Hence, the sticking coefficient $S_{c}$ and the diffusion length $\Lambda$ as a function of the surface temperature should be regarded in two regimes.

In the first regime, at low surface temperatures, the sticking coefficient $S_{c}$ is high and rather independent of the surface temperature, due to a low desorption rate from the adsorbed state. However, the diffusion length $\Lambda$ is determined by both the condensing rate and the temperature. The diffusion length increases with increasing surface temperature ( $\Lambda \propto e^{\left(-E_{d} / k T\right)}$ ), while the diffusion length $\Lambda$ decreases at higher condensing flux $\Theta_{I}$ due to a higher burial rate.

In the second regime, i.e. high surface temperatures, the sticking coefficient $S_{c}$ and the diffusion length $\Lambda$ are determined by the desorption rate from the physisorbed and chemisorbed state. The higher the temperature, the lower the sticking coefficient and the lower the diffusion length, due to a higher desorption rate.

\section{3: Nucleation and initial structure development}

Having now developed some ideas about the mechanism of adsorption and diffusion, one can proceed to discuss the thin film nucleation and its initial structure development. This section describes the odyssey of nucleation events that start with the clustering of isolated adatoms on a substrate, followed with the incorporation of additionally deposited atoms and ends after the first several layers of film formation. Since surface and interface energies $\gamma$, i.e. the characteristic free energy (per unit area) needed to create an additional piece of surface or interface, play a key role in these processes, they will be discussed first.

\section{Surface energy:}

Atoms at free surfaces or at interfaces are more energetic than atoms in the bulk, because they make fewer bonds and are thus less constrained. Consequently, the creation of a free surface involves the removal of neighbouring atoms (bond-breaking) and thus involves energy input. The surface free energy $\gamma$ may be seen as the energy $\left(\mathrm{J} / \mathrm{m}^{2}\right)$ needed to create an additional free surface per unit area. Alternatively, there will be a thermodynamic driving force for nuclei, islands, grains,... (see later for definition of these terms) to reduce their total surface energy $\gamma A$, with $A$ the surface area of the solid. This reduction in surface energy should happen by adapting the shape to the energetically most stable configuration. This can happen by means of surface diffusion or re-structuring (see later).

During the thin film growth, both $\gamma$ and $A$ are changing. The area $A$ depends on the size and shape of the nuclei, islands or grains and on the surface topography. The surface free energy $\gamma$, depends on the chemical composition, the crystallographic orientation, atomic reconstruction,... [5]. Due to this dependency, $\gamma$ is anisotropic for most crystalline solids. 
Here, the dependency of the surface free energy on the crystallographic orientation is discussed in more detail. Since the surface free energy results from the absence of neighbouring atoms, the surface free energy will be larger the more the coordination number was decreased, i.e. the more neighbouring atoms were removed [2,4,5]. A closed-packed plane in a crystalline solid maximizes the number of bonds within that plane and minimizes the number of bonds perpendicular to the plane. Hence, a closed-packed plane has less dangling bonds than any other plane and it therefore has a lower $\gamma$ than other planes. However, in solids having ionic or polar bonds, $\gamma$ also tends to be lower for planes containing equal numbers of cations and anions, since this results in charge neutrality of the surface. This bond-breaking model gives a phenomenological insight into the dependency of the surface free energy on the crystallographic orientation. The actual value of the surface free energy is often calculated by simulating the electronic structure of surfaces [15-20]. Table I.3.1. lists the planes of lowest surface energy for several crystal structures.

Table I.3.1: Planes of lowest surface energy for several crystal structures.

\begin{tabular}{|l|l|}
\hline Crystal structure & Lowest surface energy plane \\
\hline Body centered cubic (bcc) & $\{110\}$ \\
\hline Face centered cubic (fcc) & $\{111\}$ \\
\hline Hexagonal closed packed (hcp) & $\{0001\}$ \\
\hline Fluorite & $\{111\}$ \\
\hline Rocksalt & $\{100\}$ \\
\hline
\end{tabular}

Due to this dependency of $\gamma$ on the crystallographic orientation, a crystalline solid will try to adjust its shape to minimize the total surface energy:

$$
\sum_{k} \gamma_{k} A_{k}=\text { minimal }
$$

in which $k$ is the summation over all free surfaces. All surfaces are crystallographic planes and the solid shape adjustment is called faceting, because the solid will be terminated with crystallographic planes (facets).

The Wulff theorem states that when the surface energy of a crystalline solid is minimized, it will hold that $\gamma_{\mathrm{k}} / \mathrm{r}_{\mathrm{k}}$ is constant, in which $r_{k}$ denotes the perpendicular distance from the centre of the solid to the $k^{\text {th }}$ facet. This theorem is visualized in figure I.3.2. in the two dimensional case. It has been assumed that $\gamma_{100} / \gamma_{111}$ is 0.7 , so $r_{1} / r_{2}$ is 0.7 .

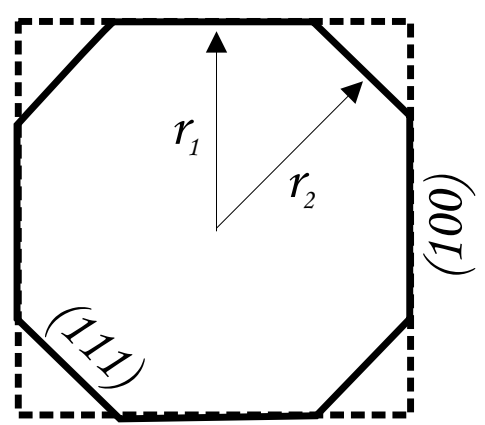

Figure I.3.2: Two dimensional example of an equilibrium solid shape (full lines) according to Wulff's theorem. The dashed lines represent the kinetically determined growth shape.

However, attention should be called to the distinction between the thermodynamic equilibrium shape of a crystalline solid according to Wulff's theorem, as discussed here, and the shape that the solid would have being grown from the vapor at a finite growth rate. In this latter case, the condensation rate is often higher on facets of high $\gamma$ because bonding is stronger. This causes a growth rate anisotropy, giving rise to a solid faceted by planes of slowest growing planes $[1,4,5]$. The fact that solids retain their kinetically determined or 
thermodynamic most stable crystal shape compels that the atom diffusion along the edges is relatively fast and that the diffusing particles are able to cross the corners. Without effective corner crossing, growth must lead to the formation of fractal like solids [21]. The equilibrium Wulff shape as well as the kinetically determined growth shape has been observed experimentally [22-25]. It has also been observed that this growth according to the Wulff equilibrium shape or the kinetically determined growth shape is already established for crystallites of 2- $5 \mathrm{~nm}$ or larger [26].

\section{Nucleation:}

To get feeling with the correlation between the surface energy and the nucleation phenomenon, a basic model for homogenous nucleation from a supersaturated vapor will be discussed first.

Assume a spherical nucleus with radius $r$, which consists of several separate atoms. It will cost energy to create a new surface: $4 \pi r^{2} \gamma$. On the other hand, the nucleus will gain energy because of the formation of a stable phase: $4 / 3 \pi r^{3} \Delta G_{v}$, in which $\Delta G_{v}$ expresses the free energy of the nucleus per unit volume and which is of course material dependent. The total energy is then the summation of the energy cost and the energy gain. The energy cost, energy gain and total change in free energy $\Delta G_{t o t}$ as a function of the nucleus radius are shown in figure I.3.3.

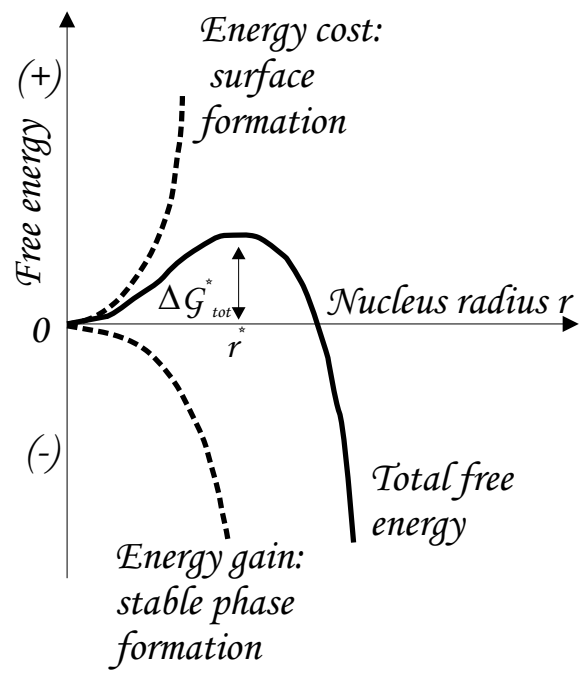

Figure I.3.3: Variation of the total free energy $\Delta G_{t o t}$ of a nucleus as a function of its radius $r$.

In this example, the free energy of the nucleus first increases, till $\Delta G_{t o t}$ goes through a maximum and then decrease with increasing radius $r$. This means that a nucleus having a radius $r$ less than that at which $\Delta G_{\text {tot }}$ goes through the maximum will spontaneously decompose. Larger nuclei are stable and will spontaneously incorporate other atoms. The critical nucleus radius $r^{*}$ at which $\Delta G_{t o t}$ is at maximum is found by $d\left(\Delta G_{t o t}\right) / d r=0$. In this way, also $\Delta G^{*}{ }_{\text {tot }}$ is found, which is the energy barrier for nucleation.

The heterogeneous nucleation of a condensed film on a substrate can be briefly discussed here in an analogous way. More detailed discussions about the nucleation phenomenon may be found in literature $[27,28]$. Since the following approach uses only the thermodynamically defined interface and surface energies, it is called the capillarity theory of nucleation $[2,3]$. A schematic drawing of the heterogeneous nucleation process of a film on a substrate is shown in figure I.3.4. 


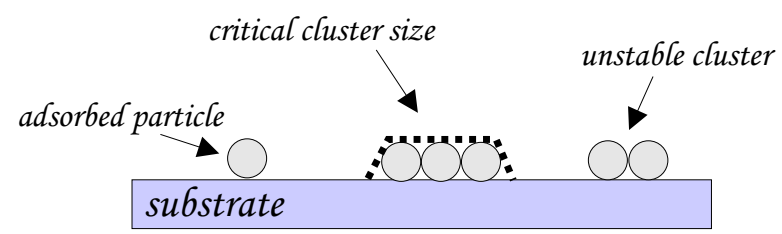

Figure I.3.4: Atomistic view of the nucleation process.

Adsorbed particles may diffuse on a bare substrate and will finally encounter other diffusing particles. These adparticles may form a cluster which will be stable if it reaches its critical nucleus size $r^{*}$. Although this nucleation process mainly relies on atomistic processes, most nucleation theories use thermodynamics.

In the following model, the shape of the nuclei is approximated by a spherical cap, as shown in figure I.3.5.

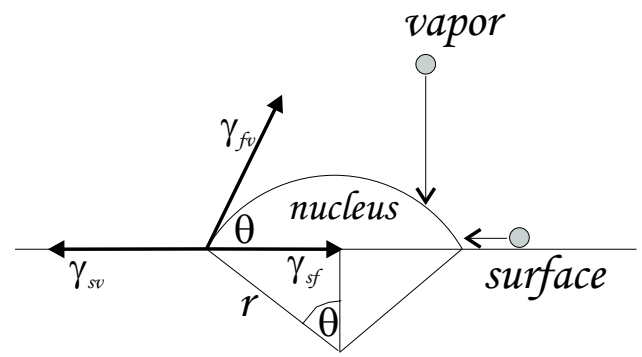

Figure I.3.5: Scheme of the nucleation process and the notations used for the capillarity nucleation theory.

The capillarity theory neglects the anisotropy of the interface and surface energies, $\gamma_{s f}, \gamma_{s v}$ and $\gamma_{f v}$ which denote the interfacial energies, identified by the subscripts $f, s$ and $v$ representing film, surface and vapor respectively. A pair of subscripts refers to the interface between the indicated phases. As seen in figure I.3.5, the vapor may arrive at the nucleus either by surface diffusion or directly from the gas phase. The latter is the dominant mode when the vapor has a very low trapping probability elsewhere on the substrate or when the desorption rate of the adsorbed particles is so high that most of them never reach a nucleus. However, it is more common for nuclei to accumulate by incorporation of diffusing adparticles.

Assuming impinging atoms on the substrate, creating a nucleus with dimension $r$, the change in total free energy of the nucleus is now expressed by:

$$
\Delta G_{t o t}=a_{3} r^{3} \Delta G_{v}+a_{1} r^{2} \gamma_{f v}+a_{2} r^{2} \gamma_{s f}-a_{2} r^{2} \gamma_{s v} \text { (eq.I.3.1) }
$$

For a spherical cap nucleus as shown in figure I.3.5, the geometric constants are

$$
a_{1}=2 \pi(1-\cos \theta), a_{2}=\pi \sin ^{2} \theta, a_{3}=\pi / 3\left(2-3 \cos \theta+\cos ^{3} \theta\right) \text { (eq.I.3.2) }
$$

in which $a_{1}$ is a measure for the surface area of the cap, $a_{2}$ for the contact area between cap and substrate and $a_{3}$ for the cap volume.

Balancing the interfacial energies for an equilibrium wetting angle $\theta$ results in Young's equation: $\gamma_{s v}=\gamma_{s f}+\gamma_{f v} \cos \theta$. $\theta$ is called the wetting angle because for $\theta=0^{\circ}$, the film wets the surface while for $\theta=180^{\circ}$, the film is completely dewetting the surface. Substituting the geometric constants and taking into account Young's equation, again an expression for the critical nucleus dimension $r^{*}$ could be derived from $d\left(\Delta G_{t o t}\right) / d r=0$. Substituting $r^{*}$ in $\Delta G_{t o t}$ would provide an expression for the nucleation energy barrier $\Delta G^{*}{ }_{t o t}$.

Before carrying out the mathematical derivation, it should be pointed out that a lot of other parameters are able to influence the nucleation phenomenon. This nucleation phenomenon will be influenced by e.g. impurities [21,29-31], flux of low-energetic ions [32, 33], preferential nucleation sites at kinks or steps at a non-perfect surface [28], stress (and strain) evolution, anisotropy in surface and interface energies, ... In order to take into account the effect of these parameters on the nucleation process, an extra energy term $\Delta E_{v}$ should be 
considered. Unfortunately, for a lot of these influences $\Delta E_{v}$ can't be expressed mathematically.

Taking into account the additional areal energy term $\Delta E_{v}$, the total energy of the nucleus is described by:

$$
\Delta G_{t o t}=a_{3} r^{3} \Delta G_{v}+a_{1} r^{2} \gamma_{f v}+a_{2} r^{2} \gamma_{s f}-a_{2} r^{2} \gamma_{s v}+a r^{2} \Delta E_{v} \text { (eq.I.3.3) }
$$

in which $a$ is $a_{1}$ or $a_{2}$ depending on the influencing parameter.

Calculation of $d\left(\Delta G_{t o t}\right) / d r=0$ provide us an expression for the critical nucleus size $r^{*}$ and the nucleation energy barrier $\Delta G^{*}$ tot:

$$
\begin{gathered}
r^{*}=\frac{2\left(a_{2} \gamma_{s v}-a_{1} \gamma_{f v}-a_{2} \gamma_{s f}-\Delta E_{v} a\right)}{3 a_{3} \Delta G_{v}}(\text { eq.I.3.4) } \\
\Delta G_{t o t}^{*}=\left(r^{*}\right)^{2}\left(a_{1} \gamma_{f v}+a_{2} \gamma_{s f}-a_{2} \gamma_{s v}+\Delta E_{v} a+a_{3} r^{*} \Delta G_{v}\right)(\text { eq.I.3.5) }
\end{gathered}
$$

In the above capillarity model for heterogeneous nucleation, it has been tacitly assumed that the self-surface diffusion of adsorbed particles is high enough to ensure a minimization of the surface free energy. However, carrying out depositions on a surface at very low temperature limits the diffusion of adsorbed particles. Hence, in this regime, the surface free energy can't be minimized by surface diffusion and the capillarity theory for nucleation is no longer valid. The impinging particles will be quenched to the surface and film growth will happen in a 'hit and stick' mode. This growth mode develops its own characteristic film structure as will be discussed in section I.5 and chapter II. Carrying out depositions at a very high deposition rate has a similar influence on the nucleation phenomenon but now due to burying the adsorbed particles instead of quenching them. A similar film structure will develop.

\section{$\underline{\text { Initial structure development: }}$}

Coming back to the equations for the critical nucleus size $r^{*}$ and the nucleation energy barrier $\Delta G^{*}{ }_{t o t}$ as derived above:

$$
\begin{gathered}
r^{*}=\frac{2\left(a_{2} \gamma_{s v}-a_{1} \gamma_{f v}-a_{2} \gamma_{s f}-\Delta E_{v} a\right)}{3 a_{3} \Delta G_{v}}(\text { eq.I.3.6) } \\
\Delta G_{t o t}^{*}=\left(r^{*}\right)^{2}\left(a_{1} \gamma_{f v}+a_{2} \gamma_{s f}-a_{2} \gamma_{s v}+\Delta E_{v} a+a_{3} r^{*} \Delta G_{v}\right)(\text { eq.I.3.7) }
\end{gathered}
$$

substituting $r^{*}$ in $\Delta G^{*}$ tot gives

$$
\Delta G_{t o t}^{*}=\left(r^{*}\right)^{2} \frac{1}{3}\left(a_{1} \gamma_{f v}+a_{2} \gamma_{s f}-a_{2} \gamma_{s v}+\Delta E_{v} a\right)=\frac{-a_{3} \Delta G_{v}\left(r^{*}\right)^{3}}{2} \text { (eq.I.3.8) }
$$

Remember that $\Delta G_{v}$ expresses the energy gain due to a stable phase formation. Hence, the sign of $\Delta G_{v}$ is always negative. This means that when $r^{*}>0$ also $\Delta G^{*}{ }_{t o t}>0$.

Substituting the geometric constants (eq.I.3.2) and using Young's equation, simplifies the expression for $r^{*}$ and $\Delta G^{*}$ tot to:

$$
\begin{gathered}
r^{*}=\frac{2\left(-\Delta E_{v} a / \pi-\left(2-2 \cos \theta-\sin ^{2} \theta \cos \theta\right) \gamma_{f v}\right)}{\left(2-3 \cos \theta+\cos ^{3} \theta\right) \Delta G_{v}}(\text { eq.I.3.9) } \\
\Delta G_{t o t}^{*}=\frac{\left(r^{*}\right)^{2} \pi}{3}\left(\left(2-2 \cos \theta-\sin ^{2} \theta \cos \theta\right) \gamma_{f v}+\Delta E_{v} a / \pi\right)(\text { eq.I.3.10) }
\end{gathered}
$$

In case of $0^{\circ}<\theta \leq 180^{\circ}$, equations I.3.9 and I.3.10 lead to positive values for $r^{*}$ and $\Delta G^{*}$ tot. A wetting angle $\theta$ larger than $0^{\circ}$ means that the film is dewetting the surface. This means that adparticles gluing together during the formation of a stable nucleus will always pile up, i.e three dimensional islands will develop [35]. Evidently, this growth mode is called the island growth or the Volmer-Weber growth and is shown schematically in figure I.3.6. 


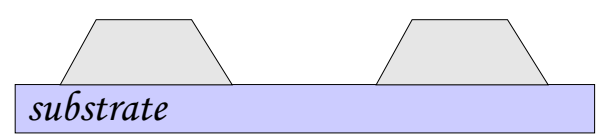

Figure I.3.6: Schematic representation of the initial growth stages for an island or Volmer-Weber growth mode.

The existence of a nucleation barrier and a critical nucleus size for a growth mode with wetting angle $>0^{\circ}$ (Volmer-Weber growth) is also conceptually in accordance with the driving force to minimize the total surface energy. A positive value for $\Delta G^{*}$ tot, together with eq.I.3.8 gives the condition

$$
\left(a_{1} \gamma_{f v}+a_{2} \gamma_{s f}-a_{2} \gamma_{s v}+\Delta E_{v} a\right)>0 \text { (eq.I.3.11) }
$$

and in the limiting case that no additional energy term is needed $\left(\Delta E_{v}=0\right)$ :

$$
\left(a_{1} \gamma_{f v}+a_{2} \gamma_{s f}\right)>a_{2} \gamma_{s v} \text { (eq.I.3.12) }
$$

Eq. I.3.12 expresses that depositing a nucleus (or a film) on a surface tends to increase the total surface energy. Hence, it is thermodynamically more stable to pile up the nucleus and to strive for an as large as possible bare surface (dewetting).

However, the wetting angle $\theta$ can also reach the limiting value of $0^{\circ}$. Calculating $r^{*}$ and $\Delta G^{*}$ tot from eq.I.3.9 and eq.I.3.10 with $\theta$ approaching $0^{\circ}$ lead to $r^{*}=0$ and $\Delta G^{*}{ }_{\text {tot }}=0$. This means that in the limiting case of $\theta=0^{\circ}$, neither a nucleation barrier nor a critical nucleation size exist. Adparticles will always nucleate on the surface and will form a single monolayer over the whole surface before starting a second monolayer since $\theta=0^{\circ}$. This film growth mode is called a layer-by-layer growth or a Frank-van der Merwe growth and is represented in figure I.3.7.

\section{substrate}

Figure I.3.7: Schematic representation of the initial growth stages for a layer-by-layer or Frank-van der Merwe growth mode.

Verification of the thermodynamic condition for the minimization of the total surface free energy, eq.I.3.8 gives for $\Delta G^{*}$ tot $\leq 0$

$$
\left(a_{1} \gamma_{f v}+a_{2} \gamma_{s f}-a_{2} \gamma_{s v}+\Delta E_{v} a\right) \leq 0(\text { eq.I.3.13) }
$$

In case that $\Delta E_{v}=0$, eq.I.3.13 leads to

$$
\left(a_{1} \gamma_{f v}+a_{2} \gamma_{s f}\right) \leq a_{2} \gamma_{s v} \text { (eq.I.3.14). }
$$

This indicates again that layer-by-layer growth is in accordance with the driving force to minimize the total surface energy, since adding a film to the substrate lowers the surface energy in case that eq.I.3.14 is fulfilled.

Up to now, it was tacitly assumed that the additional energy term $\Delta E_{v}$ is constant during the growth of the whole film. However, this is not always the case because the additional energy can change during the film growth, e.g. due to increasing stress, additional ion bombardment, increasing surface temperature,...

This brings us immediately to a third growth mode: the layer plus island or the StranksiKrastanov growth mode.

This means that the film will grow in the layer-by-layer mode as long as eq.I.3.13 is fulfilled. However, when the additional energy $\Delta E_{v}$ increased that much, it can be thermodynamically more favorable to grow further in the island mode. This layer plus island or StranskiKrastanov growth mode is schematically represented in figure I.3.8. 


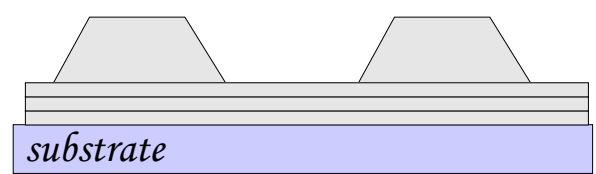

Figure I.3.8: Schematic representation of the initial growth stages for a layer plus island or Stranski-Krastanov growth mode.

So far, the additional energy term $\Delta E_{v}$ was treated in general since in most cases no mathematical expression exists for this term. However, the influence of stress (and strain) evolution on the nucleation process can be expressed mathematically and will be worked out in the next discussion.

According to Wessels et al. [34] the additional areal energy term $\Delta E_{v}$ is given by

$$
\Delta E_{v}=\left(\varepsilon^{2}-\delta^{2}\right) \mu h \text { (eq.I.3.15). }
$$

In this equation, $h$ stands for the height of the film grown in the layer-by-layer mode, $\mu$ is the elastic modulus of the film, $\varepsilon$ is the strain and $\delta$ is the lattice mismatch between the film and the substrate. The misfit $\delta$ is defined as $\left[a_{0}(s)-a_{0}(f)\right] / a_{0}(s)$ where $a_{0}(s)$ and $a_{0}(f)$ are the lattice parameters of the surface and the film respectively. Since eq.I.3.15 expresses the additional energy due to stress (and strain) between the nucleus and the underlying substrate, this areal energy term should be multiplied by $a_{2}\left(a=a_{2}\right)$.

Substituting eq.I.3.15 in eq.I.3.11 and eq.I.3.13 gives the conditions for the transition between an island and a layer-by-layer growth:

island growth if $\left(a_{1} \gamma_{f v}+a_{2} \gamma_{s f}-a_{2} \gamma_{s v}+\left(\varepsilon^{2}-\delta^{2}\right) \mu h a_{2}\right)>0$ (eq.I.3.16)

and layer-by-layer growth if $\left(a_{1} \gamma_{f v}+a_{2} \gamma_{s f}-a_{2} \gamma_{s v}+\left(\varepsilon^{2}-\delta^{2}\right) \mu h a_{2}\right) \leq 0$ (eq.I.3.17).

As already discussed, Stranski-Krastanov growth may occur when the additional energy $\Delta E_{v}$ is not constant during the film growth. Since the additional energy $\Delta E_{v}$ is linear with the film thickness $h$ (see eq.I.3.15), stress will promote the existence of the Stranski-Krastanov growth mode.

Assume that the films starts growing in the layer-by-layer mode and hence according to eq.I.3.13 that $\left(a_{1} \gamma_{f v}+a_{2} \gamma_{s f}-a_{2} \gamma_{s v}+\left(\varepsilon^{2}-\delta^{2}\right) \mu h a_{2}\right) \leq 0$.

However, with increasing film thickness the additional energy will increase and at some thickness $h$ it will be thermodynamically more favorable to nucleate islands instead of proceeding the film growth in the layer-by-layer mode (see figure I.3.9). This moment is characterized by the condition that the critical nucleus size $r^{*}$ is larger than 0 , because the film growth proceeds by the layer-by-layer growth as longs as there is no barrier for nucleation (see earlier).

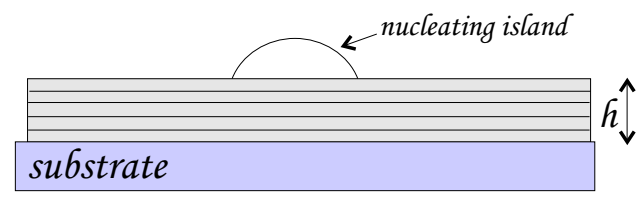

Figure I.3.9: Schematic drawing of the transition from layer-by-layer growth to island growth in the StranskiKrastanov growth mode.

Analogous to eq.I.3.6 an equation for the critical nucleus size can be given. However, it should be kept in mind that the substrate is already covered by some layers. Hence, the contribution of $\gamma_{s v}$ disappears. On the other hand, the interface energy between the film and the surface $\gamma_{s f}$ should be replaced by the interface energy between the already deposited layer and the nucleating island $\gamma_{i i}$. According to Wessels et al. [34] it can be assumed that the surface energy of the strained layer and the surface energy of the relaxed island are the same 
and hence $\gamma_{l i}=0$. This gives the following equation for the critical size of an island nucleating on an already developed layer:

$$
r^{*}=\frac{2\left(-a_{1} \gamma_{f v}-\left(\varepsilon^{2}-\delta^{2}\right) \mu h a_{2}\right)}{3 a_{3} \Delta G_{v}} \text { (eq.I.3.18) }
$$

As long as that critical nucleus size is $\leq 0$, the film will continue to grow by the layer-by-layer growth mode. However, as soon as this critical nucleus size becomes larger than zero, the growth mode will change to the island growth mode. This critical value of $r^{*}=0$ leads to the critical value of the height $h_{\text {crit }}$ :

$$
h_{c r i t}=\frac{a_{1} \gamma_{f v}}{\left(\delta^{2}-\varepsilon^{2}\right) \mu a_{2}}(\text { eq.I.3.19) }
$$

Hence, in the Stranski-Krastanov growth mode, the film will grow in the layer-by-layer growth mode until the film thickness reaches the critical height $h_{c r i t}$ and then will proceed to grow in the island growth mode.

Combination of the three growth modes leads to three stable regimes in function of the surface free energy difference and the stress development, as shown in figure I.3.10.

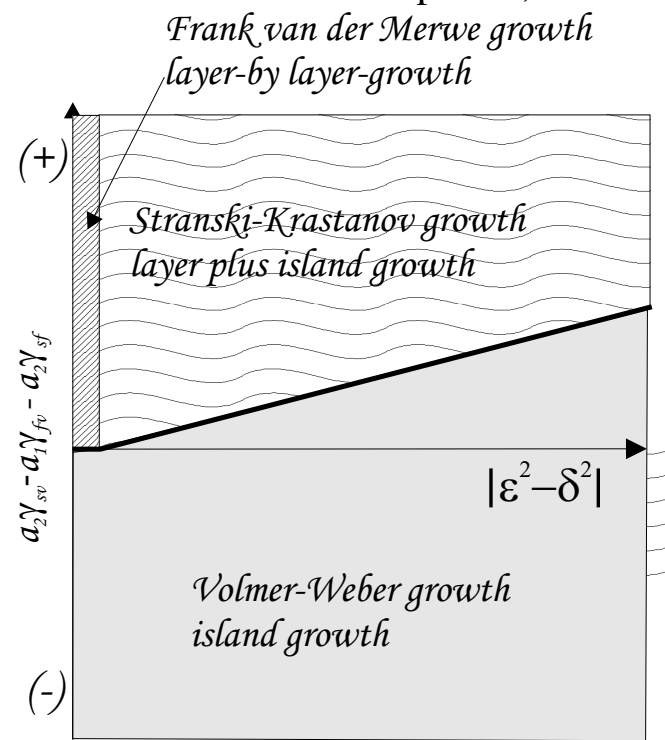

Figure I.3.10: Stability regions of the three growth modes in function of the surface energy difference and $\left|\varepsilon^{2}-\delta^{2}\right|$ which is a measure for the stress.

In case of a surface-film combination with a negligible stress $\left(\varepsilon^{2} \approx \delta^{2}\right)$ a layer-by-layer growth will develop if there is no nucleation barrier (covering the surface with adparticles lowers the surface energy). However, when there is a nucleation barrier, an island growth will develop. As seen in figure I.3.10, the range of the island growth expands when there is an additional misfit between the surface and the film, since the transition from layer-by-layer to island growth is related to $\left(\varepsilon^{2}-\delta^{2}\right)$ (eq.I.3.16 and I.3.17).

In between the layer-by-layer and the island growth, is the layer plus island growth.

As already mentioned in one of the previous sections, a lot of other parameters such as impurities, flux of low-energetic ions, preferential nucleation sites at kinks or steps at a nonperfect surface, ... will influence the nucleation process. However, these are not easy to describe mathematically, but a similar influence on the competition between the island, layerby-layer and layer plus island growth can be expected.

The actual growth mode will also depend on the competition between the thermodynamic and kinetic factors (e.g. surface temperature and condensing rate) [30, 31]. 


\section{4: Crystal and grain growth}

Film growth after nucleation implies that the islands will expand (Volmer-Weber mode) or that the film thickness will increase by adding layers (Frank-van der Merwe mode). The scope of this thesis involves the deposition of Yttria Stabilized Zirconia and Titanium Nitride on polycrystalline stainless steel or on amorphous glass, meaning a large misfit (or even no fit at all) between surface and film. Hence, all the deposited films will exhibit an island or Volmer-Weber growth mode, as discussed in the previous chapter. Therefore, only the growth evolution of the Volmer-Weber growth mode will be discussed.

The use of amorphous or polycrystalline substrates allows to isolate the effects of individual deposition variables on the thin film growth and more specific on the development of the crystallographic alignment. This because low temperature deposition on amorphous or polycrystalline substrates leads to the nucleation of islands with random orientation (i.e. without correlation to the substrate) [36], in contrast to epitaxial growth.

Distinction should be made between the crystal growth and re-structurative grain growth $[29,30]$. Islands grow as discrete islands dispersed on the substrate when they are growing independently of each other, disregarding the fact that their adparticle collection areas may overlap. This growth of discrete islands is called crystal growth, since these islands will mostly exhibit a crystalline structure. The islands which are already parts of the polycrystalline film may endure an interaction of neighbouring grains. This interaction between the different islands leads to the evolution or re-structuring of several islands into one grain. Therefore, the growth of interacting islands is called re-structurative grain growth. A more detailed discussion about re-structurative grain growth in the three growth modes can be found in [35].

\section{Crystal growth:}

The different processes during crystal growth are discussed in figure I.4.1.

During the crystal growth, it is assumed that particles are located at their final location as soon as they are chemisorbed and incorporated in the nucleus. Hence, once adparticles are chemisorbed and incorporated into the growing film, they will not change their position and the formed grain will not change its properties (e.g. crystallographic orientation) anymore.
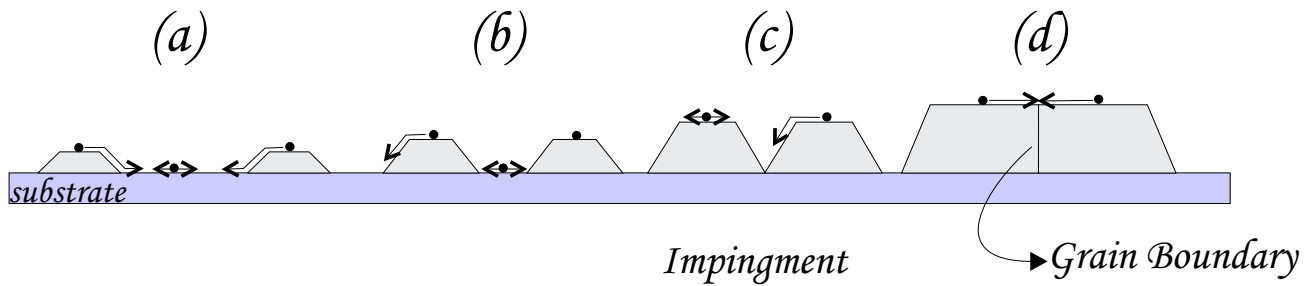

Figure I.4.1: Several processes occurring during the crystal growth.

Particles impinge and adsorb on the bare surface and on already formed nuclei. Particles initially condensing on the bare substrate will diffuse towards an existing nucleus where they possibly get chemisorbed or they diffuse along the bare substrate until they meet other diffusing adparticles to eventually form a new stable nucleus.

Particles condensing initially on an existing nucleus may diffuse around on the existing nucleus and eventually get chemisorbed on that nucleus itself. However, these particles are also able to diffuse away of the nucleus downwards to the bare substrate and even towards nearby nuclei until they are chemisorbed. These processes are shown in figure I.4.1. (phase a). 
In this way, the respective nuclei will expand to larger islands (phase b) by incorporating adparticles condensing directly from the vapor and by the flux of adparticles adsorbed on the bare substrate and diffusing to the nuclei.

This expansion of the islands proceeds until several islands are that large that they touch. The moment that the different islands touch each other is called to moment of impingement (phase c). From this moment of impingement, the surface is fully covered by the film and incoming particles are only able to condense on already existing islands (which are called grains from now on). However, a particle condensing on one grain is still able to diffuse of that grain towards the groove between both grains. The particle can chemisorb on the initial grain, in the groove or even on the neighbouring grain.

Further growth results in further expansion of the grains and filling up of the grooves between them. From now on, the different grains are separated by a grain boundary. Despite the existence of that grain boundary, the diffusion of adparticles between neighbouring grains does not cease (phase d).

As will be discussed in the next section ( $§ I .5)$, a competitive growth of neighbouring grains is only possible in an evolutionary way.

\section{$\underline{\text { Re-structurative grain growth: }}$}

With increasing temperature or mobility, atoms that were stable at lower temperatures can become active. Examples include detachment of an atom from a step onto a terrace, ejection of an atom from a kink site, removal of a corner atom of an island,...[21]. These events lead to a new phenomenon: re-structurative grain growth and may happen before, during and after the impingement. It should be mentioned that re-structurative grain growth happens in the already deposited structure.

This re-structurative grain growth phenomenon is driven by the differences in the average energy per atom for islands or grains of different sizes.

Assuming an isotropic surface and interface energy, the energy per atom will scale with the island surface to volume ratio, so that atoms in small islands will have higher energies as compared to those in larger islands [3, 5, 37, 38]. Therefore, the re-structurative grain growth leads to the shrinkage of small islands in favor of the large islands. It is expected that the average grain size increases but that the shape of the grain size distribution will be time independent. Re-structurative grain growth with these characteristics is called normal restructurative grain growth $[37,38]$.

However, the surface and the interface energies are not isotropic but a function of the crystallographic orientations, as mentioned in section I.3. Therefore, the re-structurative grain growth not only drives to the minimization of the surface curvature but also to the lowest surface and interface energy. It means that a small island can be favored over a large island despite the surface curvature effect if the surface energy of the small island is much lower. This kind of re-structurative grain growth process leads to an evolution of the distributions of island orientations as well as island sizes, favoring islands with orientation of minimum surface and interface energy. This effect is called abnormal re-structurative grain growth and often dominates the normal grain growth. Hence, re-structurative grain growth can be an initial driving force for a preferential out-of-plane oriented polycrystalline film (i.e. a nonrandom crystallographic orientation perpendicular to the substrate, but with a random crystallographic orientation parallel to the substrate) $[39,40]$.

Special attention should be paid to the possible influence of impurity drag on the development of the re-structurative grain growth. Impurities (or intentional additives) with low solubility 
will segregate to surfaces and grain boundaries. This selective segregation can limit the surface mobility and the grain boundary mobility, and therefore influences the re-structurative grain growth process $[29,30]$.

For clarity, the several re-structurative grain growth processes will be discussed one by one.

\section{$\underline{\text { Re-structurative grain growth processes: }}$}

- An interaction between neighbouring islands in which one island grows or "ripens" at the expense of another island by exchanging atoms may already happen prior to impingement of the islands. This exchange implies the adparticle detachment from an island, diffusion across the surface and attachment to another island. This process is schematically shown in figure I.4.2. in which the different atoms forming the nuclei are represented by circles. The black circles represent the atoms that detach from the left nucleus, diffuse towards the right nucleus and attach there. Since atoms are detached from one island and get attached to another island, these atoms will also change their bonding, what justifies the term "re-structurative grain growth".

(a)

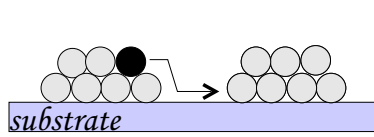

(6)

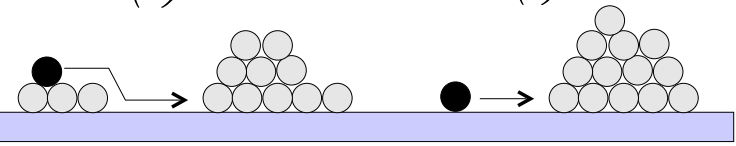

Figure I.4.2.: Atomistic view of the ripening process. The dark atoms diffuse from the left to the right island.

Depending on the limiting process (adatom detachment or diffusion) the potential energy barrier for the ripening process $E_{\text {rip }}$ is given by: $E_{\text {rip }}=E_{f}+E_{\mathrm{det}}+E_{d}$ or $E_{\text {rip }}=E_{f}+E_{d}$ respectively. $E_{f}$ is the adatom formation energy, $E_{d}$ is the surface diffusion barrier, $E_{d e t}$ is the attachment or detachment energy and $E_{s c h}$ represents the Ehrlich-Schwoebel barrier. This Ehrlich-Schwoebel barrier $E_{s c h}$ is the energy barrier that an adatom has to overcome when descending a step, because of the temporary lower number of nearest neighbours. Figure I.4.3. shows these potential energy barriers involved in the adatom detachment and diffusion process.

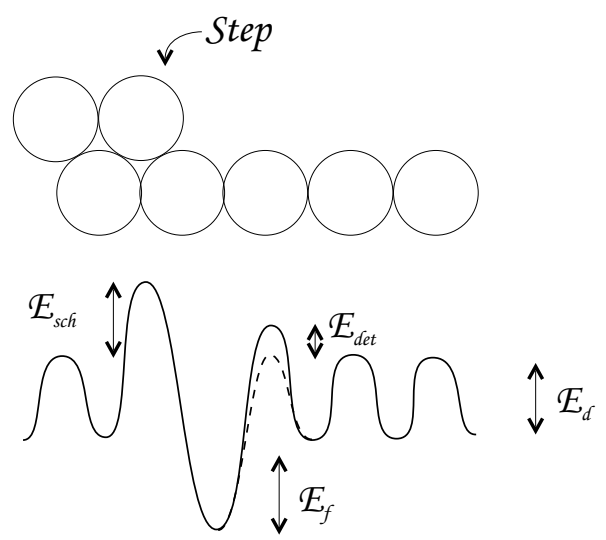

Figure I.4.3.: Schematic diagram of the potential energy barriers for the adatom formation and diffusion process near an island step edge.

This ripening process has been observed experimentally [41-44] and it has been measured by Kodambaka et al. [42] that the energy barrier $E_{\text {rip }}$ on TiN[111] is $3.5 \mathrm{eV}$ and on TiN[100] 3.4eV. 
- Re-structurative grain growth may also happen due to cluster migration. This means that the grain growth does not happen by exchanging the atoms one by one, but that the cluster as a whole is mobile and diffuses towards the other island [45]. The coalescence occurs as a result of collisions between the cluster and the island [3]. This coalescence is a liquid-like process and is schematically shown in figure I.4.4. It seems evident that the smaller the clusters the faster the cluster migration.

(a)

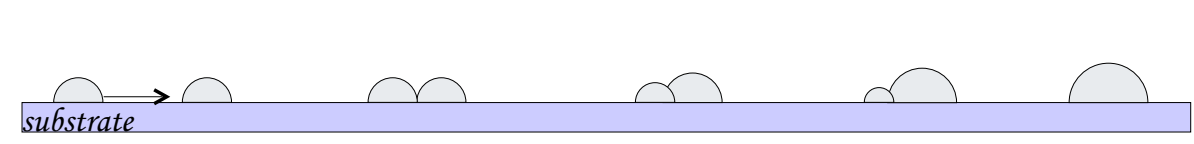

Figure I.4.4.: Schematic diagram of the coalescence after cluster migration.

- Grain boundary migration is the last possible mechanism for re-structurative grain growth. When no re-structurative grain growth occurred before island impingement (or when it was not finished), two grains will be separated by a grain boundary as also seen in figure I.4.1. (phase d). However, when the mobility is high enough, some atoms will be able to detach from the original grain and get incorporated in the other grain. They don't have to diffuse but they only have to change their bonding to become a part of the other grain. This is shown in figure I.4.5. where two grains are separated by a grain boundary and in which the different properties of the grains are represented by the different colors of the atoms.
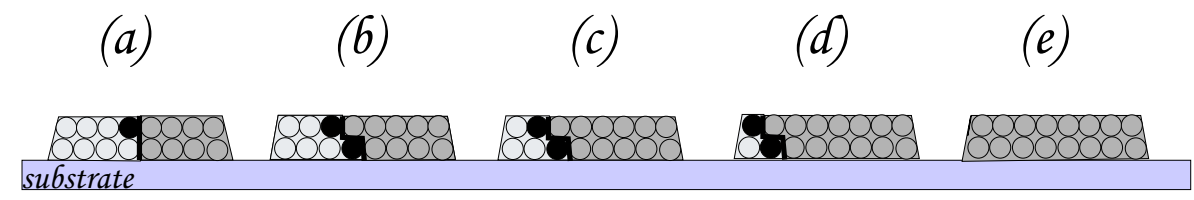

Figure I.4.5: Atomistic view of the grain boundary migration process. The black atoms are migrating "through" the grain boundary and hence are moving it to the left.

By changing its properties, it looks like one grain is growing in favor of another grain, while in fact only the grain boundary is shifting. The energy barrier for this grain boundary diffusion is in the order of the energy barrier for ripening since the atoms also have to detach and attach again.

If the re-structurative process occurs, the fraction of the surface that is covered by islands and their surrounding collection areas possibly decreases. This can lead to a situation in which relatively late in the film growth process new areas of the surface are exposed to the vapor and in which nucleation can occur again. This phenomenon is known as secondary nucleation [38].

At still higher surface temperature or mobility, diffusion and re-structurative growth can also occur within the bulk of the thin film. Then, this process is called bulk diffusion and gives rise to a specific thin film microstructure as will be discussed in the next section and in chapter II. Generally, the activation energies for bulk diffusion are higher than those for surface diffusion (see table I.4.6) [46].

Table I.4.6: Bulk diffusion and surface diffusion activation energies for several materials [46].

\begin{tabular}{|l|l|l|}
\hline Material & $\begin{array}{l}\text { Bulk diffusion } \\
\text { activation energy }(\mathrm{eV})\end{array}$ & $\begin{array}{l}\text { Surface diffusion } \\
\text { activation energy }(\mathrm{eV})\end{array}$ \\
\hline $\mathrm{Cu}$ & 2.16 & 0.57 \\
\hline $\mathrm{Ni}$ & 2.96 & 0.91 \\
\hline $\mathrm{Pd}$ & 2.77 & 0.91 \\
\hline $\mathrm{Mo}$ & 4.01 & 0.3 \\
\hline
\end{tabular}




\section{5: Further film growth}

The final structure and morphology of a film strongly depends on the presence or absence of the re-structurative grain growth since this is connected with the mobility or immobility of the grain boundaries, as discussed in the previous section.

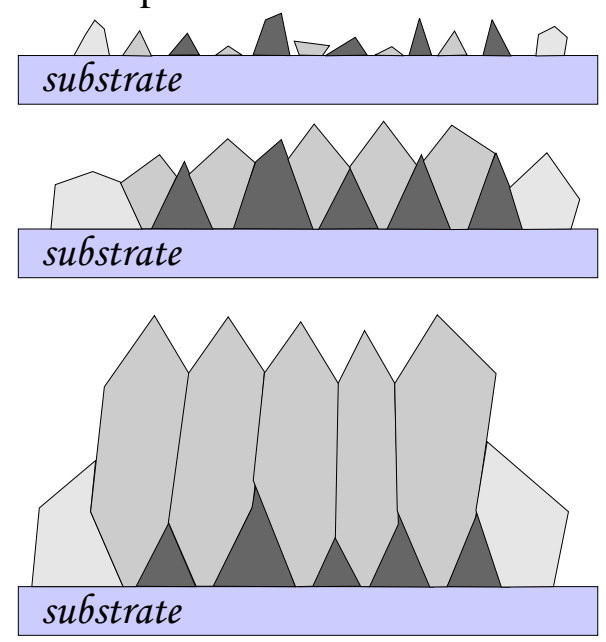

Figure I.5.1.: Scheme of the evolution of the structure and morphology of a film grown at conditions where the grain boundaries are immobile (i.e. no re-structurative grain growth happens). The different colors represent grains with a different crystallographic orientation perpendicular to the substrate.

If the grain boundaries formed through island impingement are immobile (i.e. if only crystal and no re-structurative grain growth occurs) the grain structure resulting from the nucleation, growth and impingement is retained at the base of the film. This is represented in figure I.5.1. Obtaining a polycrystalline structure and a Volmer-Weber growth mode usually implies that subsequent thickening of the grains occurs through local epitaxial growth on these grains and a columnar structure develops. Since all grain boundaries are immobile, a growth competition between neighboring grains or columns can only happen in an evolutionary way. The driving force for this evolutionary growth competition is usually an anisotropy in growth rate perpendicular to substrate. This anisotropy in growth rate can be a consequence of a different sticking probability, adparticle surface mobility, adparticle potential energy, re-sputter rate, shadowing effect,... [39,47]. The influence of several deposition conditions on the anisotropy in growth rate will be discussed in more detail in chapter II and V.

If the presence of the grain boundaries not limits the diffusion of an adparticle, then the mobility of the adparticles is high enough to aim for the kinetically determined growth shape (§1.3). Growing according to the kinetically determined growth shape invokes that the islands and grains will be terminated by planes of slowest perpendicular growth rate. This implies that the grains or the top of the columns will be faceted. These faceted column tops result in a surface roughness which increases with film thickness.

However, when the grain boundaries are mobile (i.e. re-structurative grain growth may occur) several islands or grains will disappear while others will enlarge. Hence, the grain structure evolves during the initial growth and continues to evolve during further growth. This often results in a structure in which the grain boundaries penetrate through the thickness of the film, so that the average in-plane size of the columns is uniform through the thickness of the film, as shown in figure I.5.2. As already mentioned, due to the abnormal grain growth, grains with the lowest energy plane parallel to the substrate will be favored invoking a polycrystalline film with a preferential out-of-plane orientation.

Here, faceting causes the column tops to be finished with a plane of lowest surface energy. Therefore, if perfect faceting would occur, all column tops would be atomically flat and parallel to the substrate. However, these column tops usually seems not to be terminated by a 
perfect horizontal plane but seems to end with a smoothly curved surface or domes.

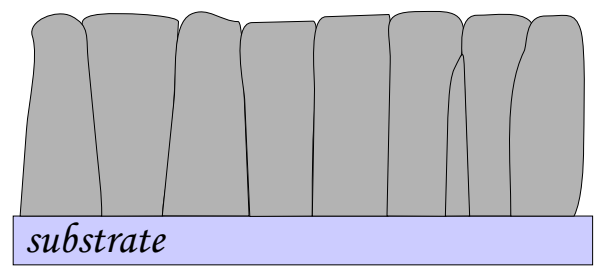

Figure I.5.2: Schematic drawing of the morphology and structure of a film grown in conditions where the grain boundaries are mobile (i.e. re-structurative grain growth can occur). No evolutionary overgrowth is visible, but all columns transverse the whole thickness of the film.

Because bonding at grain boundaries is always weaker than in the bulk of a crystalline grain, there will always be an interfacial energy $\gamma_{i}$ at the boundary between the columns (see figure I.5.3). Therefore, the columns do not completely flatten as a result of the surface diffusion, but instead approach an equilibrium shape such that the vertical components of $\gamma_{1}$ and $\gamma_{2}$ (the surface energies of grain 1 and 2 respectively) balance $\gamma_{i}$. Hence, a groove develops between two columns. Due to a high surface mobility and the mobility of the grain boundaries, these grain boundaries will not widen and a dense columnar structure develops.

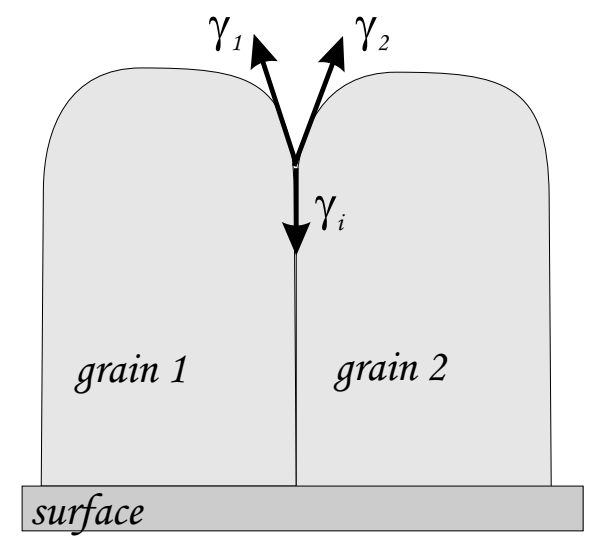

Figure I.5.3: Geometry of the top of dome shaped columns, with the corresponding interface and surface energies.

It should be mentioned that the mechanism as shown in figure I.5.3, i.e. balancing the grain boundary energy $\gamma_{i}$ by the formation of a groove between two neighbouring columns is often not strong enough to clarify the doomed or curved shape of the column tops. That doomed or curved shape of the columns is rather expected to evolve from a preferential nucleation at the middle of the column, due to e.g. the presence of a defect or dislocation.

A more detailed discussion about the evolution of the final structure and morphology of the film in function of several parameters is found in chapter II. 


\section{References}

1: A. Zangwill in "Physics at surfaces" published by Cambridge University Press ISBN: 0521347521

2: H.Lüth in "Solid surfaces, interfaces and thin films" fourth edition published by Springer ISBN: 3540423311

3: M. Ohring in "Materials science of thin films: deposition and structure" second edition published by Academic Press ISBN: 0125249756

4: J.B. Hudson in "Surface Science: an introduction" published by John Wiley \& Sons ISBN: 0471252395

5: D.L. Smith in "Thin film deposition: principles \& practice" published by McGraw-Hill ISBN: 0070585024

6: X.-C. Guo, J.M. Bradley, A. Hopkinson, D.A. King: Surf. Sci. 310 (1994) p.163-182

7: A.C. Luntz, M.D. Williams, D.S. Bethune: J. Chem. Phys. 89 (7) (1988) p.4381-4395

8: M.D. Williams, D.S. Bethune, A.C. Luntz: J. Chem. Phys. 88 (4) (1988) p.2843 -2845

9: G.R. Darling, S. Holloway: Surf. Sci. 304 (1994) L461-467

10: L. Osterlund, I. Zoric, B. Kasemo: Phys. Rev. B 55 (23) (1997) p.15 452-15 455

11: L. Vatuone, C. Boragno, M. Pupo, P. restelli, M. Rocca, U. Valbusa: Phys. Rev. Lett. 72 (4) (1994) p.510-513

12: A. Raukema, D. A. Butler, F.M.A. Box, A. W. Kleyn: Surf. Sci. 347 (1996) p.151-168

13: A. Hodson, A.K. Lewin, A. Nesbitt : Surf. Sci. 293 (3) (1993) p.211-226

14: A. Nesbitt, A.K. Lewin, A. Hodson: J. Phys.:Cond. Matter 3 (1991) S71-S76

15: D. Gall, S. Kodambaka, M.A. Wall, I. Petrov, J.E. Greene: J. Appl. Phys. 93 (11) (2003) p.9086-9094

16: C.L. Liu, J.M. Cohen, J.B. Adams, A.F. Voter: Surf. Sci. 253 (1991) p.334-344

17: J. Zhang, F. Ma, K. Xu: Appl. Surf. Sci. 229 (2004) p.34-42

18: Z. Xiong, S. Shi, C. Ouyang, M. Lei, L. Hu, Y. Ji, Z. Wang, L. Chen: Phys. Lett. A 337 (2005) p.247-255

19: C.V. Ciobanu, D. T. Tambe, V. B. Shenoy: Surf. Sci. 582 (2005) p.145-150

20: N. Ooi, J. B. Adams : Surf. Sci. 574 (2005) p.269-286

21: Z. Zhang, M.G. Lagally: Science 276 (5311) (1997) p.377-383

22: J.C. Heyraud, J.J. Metois: Surf. Sci. 128 (1983) p.334-342

23: C.H. Choi, L. Hultman, R. Ai, S.A. Barnett : Appl. Phys. lett. 57 (1990) p.2931-2933

24: S.N. Jacobsen, U. Helmersson, R. Erlandsson, B. Skarman, L.T. Wallenberg: Surf. Sci. 429 (1999) p.22-33

25: M.R. Castell: Phys. Rev. B 68 (2003) p.235411.1 -235411.8

26: L.D. Marks: Rep. Prog. Phys. 57 (1994) p.603-649

27: J.A. Venables, G.D.T. Spiller, M. Hanbücken : Rep. Prog. Phys. 47 (1984) p. 399-459

28: C. Ratsch, J. Venables: J. Vac. Sci. Technol. A 21(5) (2003) s96-s109

29: P.B. Barna, M. Adamik: Thin Solid Films 317 (1998) p.27-33

30: P.B. Barna, M. Adamik in "Science and Technology of thin films" published by World scientific ISBN: 9810221932

31: R.Q. Hwang, C. Günther, J. Schröder, S. Günther, E. Kopatzki, R.J. Behm: J. Vac. Sci.

Technol. A10(4) (1992) p.1970-1980

32: J.E. Greene in "Handbook of crystal growth vol.1" published by Elsevier ISBN: 0444 889086

33: J.E. Greene, S.A. Barnett, J.E. Sundgren, A. Rockett in "Plasma-Surface Interactions and Processing of Materials" Nato Series, Series E: Applied Sciences Vol(176) published by Kluwer Academic ISBN: 0792305841

34: B.W. Wessels: J. Vac. Sci. Technol. B 15(4) (1997) p.1056-1058 
35: M. Zinke-Allmang: Thin Solid Films 346 (1999) p.1-68

36: C.V. Thompson, R. Carel: Mater. Sci. Eng. B32 (1995) p.211-219

37: C.V. Thompson, R. Carel: J. Mech. Phys. Solids. 44 (5) (1996) p.657-673

38: C.V. Thompson: Annu. Rev. Mater. Sci. 30 (2000) p.159-190

39: Y.Kajikawa, S. Noda, H. Komiyama: J. Vac. Sci. Technol. A 21(6) (2003) p.1943-1954

40: I.Petrov, P.B. Barna, L. Hultman, J.E. Greene: J. Vac. Sci. Technol. A 21(5) (2003) S117S128

41: B.K. Chakraverty: J. Phys. Chem. Solids. 28 (1967) p.2401-2412

42: S. Kodambaka, V. Petrova, A. Vailionis, P. Desjardins, D. G. Cahill, I. Petrov, J.E. Greene: Thin Solid Films 392 (2001) p.164-168

43: J.B. Hannon, C. Klünker, M. Giesen, H. Ibach: Phys. Rev. Lett. 79 (13) (1997) p.25062509

44: W. Theis, N.C. Bartelt, R.M. Thromp: Phys. Rev. Lett. 75 (18) (1995) p.3328-3331

45: B. Voigtländer, T. Weber: Phys. Rev. Lett. 77 (18) (1996) p.3861-3864

46: M.Prutton in "Introduction to surface physics" published by Oxford science publications ISBN 0198534760

47: G.H. Gilmer, H. Huang, T. Diaz De La Rubia, J. Dalla Torre, F. Baumann: Thin Solid Films 365 (2000) p.189-200 


\section{Chapter II: Structure Zone Models.}

\section{1: Introduction}

The sequential substeps during thin film growth and their underlying principles which determine the structure and morphology of a film were described in chapter I. It was concluded that sputter deposition of YSZ and TiN on a polycrystalline or amorphous substrate will result in a film with an island or Volmer-Weber growth mode. Depending on the mobility during the growth, several diffusion processes or fundamental phenomena of structure formation such as nucleation, crystal growth or even re-structurative grain growth may determine the final film structure. These are quantified by the characteristic diffusion and activation energies whose magnitudes scale directly with the melting point of the deposited material, at least for materials of a given class (e.g. fcc metals, transition metals, semiconductors, oxides and nitrides,...) [1]. Beside the mobility also other parameters such as the deposition rate, impurities, energetic bombardment,... were mentioned to influence the film growth and its final structure.

The influence of all these parameters on the structural features that develop in the deposited films were depicted in terms of structure zone models (SZM's) by many authors [2-18]. At first, the basic structure zone model (BSZM) will be described. This BSZM interprets the film structure at various temperatures, taking into account the influence of the elementary process of diffusion and the fundamental phenomena of structure formation (nucleation, crystal growth and re-structurative grain growth), however without considering the effects of impurities.

Since the co-deposition of impurities is hardly avoidable in real films, a revised structure zone model (RSZM) was proposed by Barna et al. [13-15] and will be described secondly.

\section{2: Basic structure zone model}

Although nearly all published SZM's use the terminology introduced by Movchan and Demchishin [2] (zone I, II and III) and by Thornton [7] (zone T), this terminology has not been used consistently throughout these publications. Also here, the widely accepted terminology (zone I, T, II, III) will be used. However, the following discussion of these four zones should eliminate doubts about the microstructural and underlying physical characteristics of each zone. At the end of this chapter a diagram is summarizing the here described BSZM, the main characteristics of each zone and the terminology of these zones used by other authors. Further in this thesis, many experimental results and discussions will refer to this diagram.

\section{Zone I:}

As mentioned in the introduction, the BSZM interprets the film structure mainly in function of the temperature. Since the elementary processes depend on the corresponding activation energies -whose magnitudes scale directly with the melting point of the deposited materialthe homologous temperature $T$ will be used. This homologous temperature is the ratio of the substrate temperature $T_{s}$ to the melting temperature of the deposited material $T_{m}$ (i.e. $T=$ $\left.T_{s} / T_{m}\right)$.

\section{Zone Ia:}

At very low temperature $T$, the adparticles will have no mobility since they can't overcome the diffusion barriers $E_{d, p h y s}$ or $E_{d, c h e m . ~}$ (see chapter I, §I.2). This means that the adparticles will stick at the growing film at the same place where they hit it. This is known as hit-and-stick 
growth or also as "ballistic" deposition since the only motion of the particles which affects the growth is the incoming direction of the material flux [19]. As already mentioned in chapter I (§I.3 section nucleation), the adparticles will not be able to nucleate into crystalline islands when there is no mobility or diffusion at all. Hence, an amorphous-like structure will develop. Even with a uniformly and perpendicularly arriving material flux on a perfect smooth substrate, this ballistic or hit-and-stick growth gives no rise to a smooth nor to a homogenous film. On the contrary, a columnar and rather rough film develops mainly due to statistical roughening and self-shadowing [20]. Because of the finite size of the adparticles, film growth can result in overhang structures as shown in figure II.2.1.a, which shadow the lower areas from deposition. Hence, even in case of a perpendicular deposition, a structure of columns develops with shadowed voids between them (see figure II.2.1.b). This columnar structure, separated by voids has been simulated by Müller et al. [21-23], Leamy et al. [24] and Gilmer [25]. Due to the voided structure, the density of the deposited film is much smaller than the density of the bulk material.

When the material flux is arriving obliquely at an angle $\alpha$, it causes the columns to be tilted towards the incoming material flux by an angle $\beta$ (see figure II.2.1.c). Due to the absence of diffusion and the finite size of the adparticles, $\alpha$ and $\beta$ are often related by the tangent-rule: $\tan \beta=1 / 2 \tan \alpha[24-26]$.
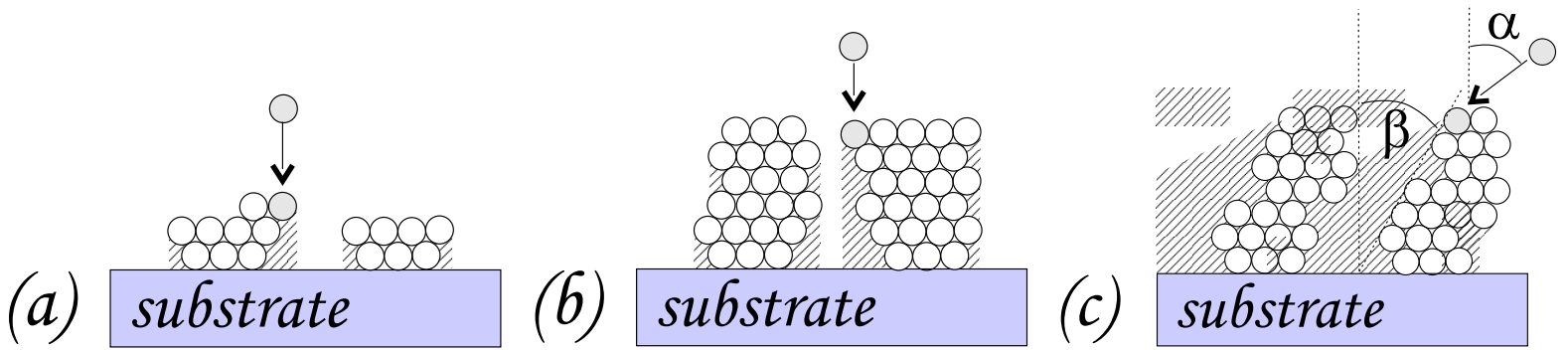

Figure II.2.1: Atomistic view of the growth on a substrate at very low temperature (zone Ia). (a) finite size effect on shadowing, (b) columnar growth with voids between them, (c) tilt angle $\beta$ of the columns when the material flux is tilted by an angle $\alpha$. The shaded area is the shadowed region.

Up to now, it was tacitly assumed that the incoming material flux is arriving along one single direction. However, for many deposition techniques such as magnetron sputtering, this material flux usually has a large angular spread (see chapter VII). Evidently, the larger the angular spread on the incoming material flux, the larger the influence of shadowing on the voided structure.

Finally, it should be mentioned that as soon as the adparticles have a small probability to diffuse, the film will not be completely amorphous-like anymore but some small crystallites can nucleate. Since diffusion and nucleation are statistical processes and due to the very small mobility, the crystals will not infinitely increase in size but the columns will exist of small equiaxed grains. Crystalline grains are separated by amorphous-like regions.

Figure II.2.2 shows a schematic drawing of a film grown at a low temperature, resulting in a structure as described above. Such a structure is called to be zone Ia.

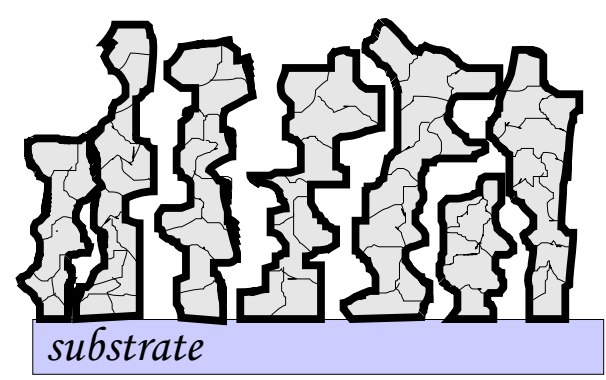

Figure II.2.2: Schematic drawing of the resulting structure of a film grown at zone Ia conditions. A voided structure, with the columns amorphous-like or consisting of small grains. 
Since the films deposited at zone Ia conditions are amorphous-like, the films will not tend to have one specific crystallographic direction perpendicular to the substrate. This means that no preferential out-of-plane orientation will develop in these films.

It should be noticed that a zone Ia structure can also be obtained at higher temperatures. Therefore, the deposition rate should be that high that -although surface diffusion is allowedadparticles are buried by the next deposited adparticles before they can diffuse to a more stable place. Although this is not a hit-and-stick growth, but rather a "burial" growth, also a zone Ia structure is obtained.

\section{Zone Ib:}

A deposition carried out in zone Ia regime, but continuously bombarded by energetic species will exhibit another structure. More precisely, due to the energetic bombardment, knock-on events may occur on the overhang structures. Such a knock-on event is shown in figure II.2.3 a and has been simulated by Müller et al. [21,23,27] and Gilmer [25]. This means that the voids will be filled up by particles and a more dense columnar structure will evolve, i.e. the zone Ib structure. When the different columns are no longer separated by voids, the term grain boundary should be used. A schematic drawing of the resulting structure is shown in figure II.2.3.b.

(a)

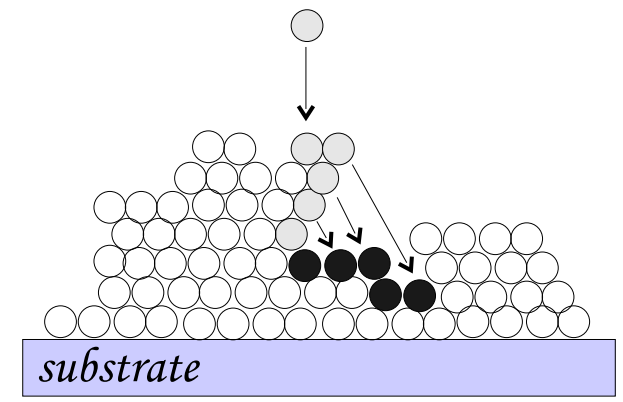

(b)

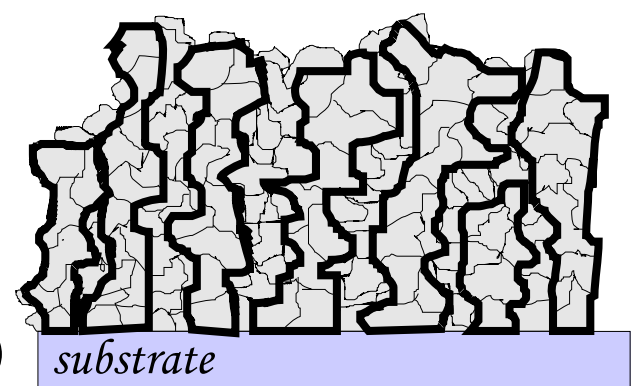

Figure II.2.3: A knock-on event and its consequence for the film structure (a) and a schematic drawing of the resulting structure for a zone Ib deposition (b).

The energetic bombarding species can be accelerated ions, fast neutrals or in case of sputtering also sputtered particles and reflected and neutralized ions.

The only difference between zone Ia and zone $\mathrm{Ib}$ is that the film is bombarded by energetic particles and hence kinetic energy is delivered to the film. It must be noticed that it is impossible to pass from zone Ia into zone Ib by increasing the temperature (i.e. increasing the thermal-induced mobility). This because increasing the thermal-induced mobility not only results in filling-up the voids but also leads to other changes (see zone Ic).

Messier ${ }^{*}$ et al. [6] worked out a SZM in which the transition from zone Ia to zone Ib in function of the delivered kinetic energy is described in more detail.

Since the thermal-induced mobility is not increased, the film is still amorphous-like or consists of small equiaxed crystalline grains. Hence, still no preferential out-of-plane orientation will develop.

\section{Zone Ic:}

When the deposition is carried out at higher temperature $T$, the thermal-induced mobility increases and the adparticles are able to nucleate into crystalline islands. Due to the increased temperature, the adparticles will be able to overcome the diffusion barriers $E_{d, p h y s}$ or $E_{d, c h e m}$, what means that the adparticles are able to diffuse around on the underlying crystals or grains. Hence, each island or grain will be able to grow according to its kinetically determined

\footnotetext{
* The here mentioned zone Ia and zone Ib are in the terminology of Messier zone I and zone T (see table II.5 at the end of this chapter).
} 
growth shape (see chapter I, §I.3). Therefore, in contrast with zone Ia and zone Ib, where the mobility was too low to aim for that crystal habit, all grains in zone Ic will be faceted. Due to an anisotropy in perpendicular growth rate of the different crystallographic planes, this faceting during the film growth occurs in such a way that the grains are terminated by the planes of lowest perpendicular growth rate. This because only the planes with lowest growth rate survive, since the faster growing planes extinguish themselves, as shown in figure II.2.4.

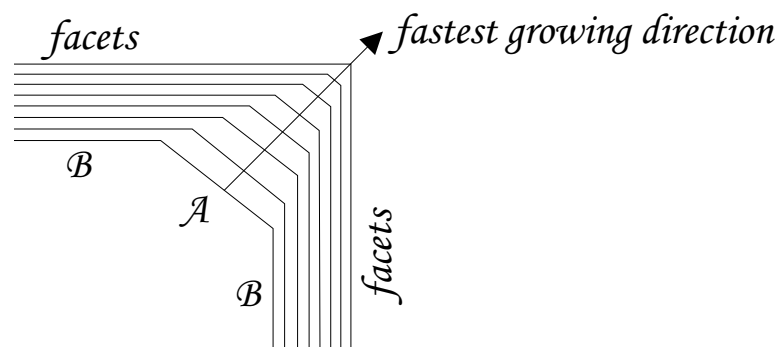

Figure II.2.4: Schematic drawing of the evolution to the kinetically determined growth shape in a two dimensional representation. In this example, plane A has a larger perpendicular growth rate than plane B.

The perpendicular growth rate of a crystallographic plane is influenced by many parameters such as re-sputter rate, impurity contribution, sticking coefficient, adparticle mobility, .... The influence of the re-sputter rate can be neglected in deposition conditions where an energetic bombardment is absent. Also the influence of the impurities on the perpendicular growth rate can be neglected, when carrying out the deposition in pure conditions. The influence of the two remaining parameters, i.e. sticking coefficient $S_{c}$ and adparticle mobility, on the perpendicular growth rate $R_{d} \perp$ of a plane is shown in figures II.2.5 and II.2.6 respectively.

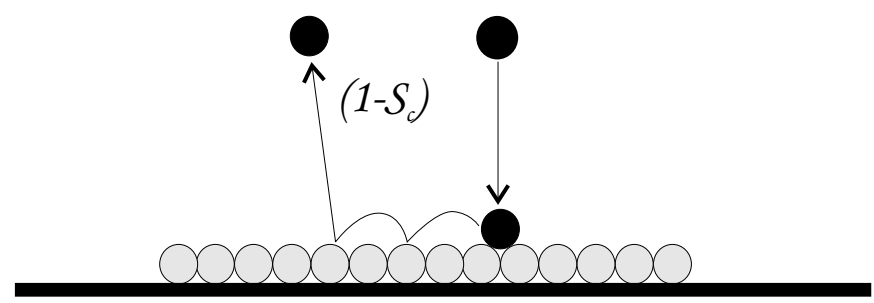

Figure II.2.5: Influence of the sticking coefficient $S_{c}$ on the perpendicular growth rate $R_{d} \perp$ of a crystallographic plane. The black particles represent adatoms. The larger the sticking coefficient, the larger the perpendicular growth rate.

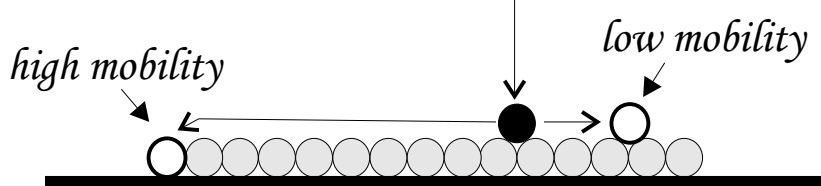

Figure II.2.6: Influence of the adparticle mobility on the perpendicular growth rate $R_{d} \perp$ of a crystallographic plane. The black particles represent adatoms. The larger the mobility, the lower the perpendicular growth rate.

-Evidently, the larger the sticking coefficient, the larger the perpendicular growth rate of a crystallographic plane [17]. According to the Hartman-Perdok theory [28], the sticking coefficient $S_{c}$ depends on the number of dangling bonds or nearest neighbours offered to the adparticle. The larger the number of nearest neighbours offered to the adparticles, the larger the sticking coefficient $S_{c}$. Hence, to know which crystallographic plane will have the lowest growth rate and will become the resulting facet, it should be known which plane offers the lowest number of nearest neighbours to the incoming adparticles. 
- Also the adparticle mobility influences the perpendicular growth rate of a plane. As shown in figure II.2.6, an adparticle can reach the edge of the crystal and can descend the step, at least if the mobility of the adparticle is high enough. Hence, if the mobility is high enough, these adparticles will contribute to the lateral growth rate instead of the perpendicular growth rate. When the mobility of the adparticles is low, nearly no adparticles will descend the step and all adparticles will contribute to the perpendicular growth rate. Hence, the higher the mobility of the adparticles, the larger the lateral and the lower the perpendicular growth rate of the crystal.

Huang et al. [29] pointed out that also the mobility of the adparticles linearly depends on the number of nearest neighbours offered to these adparticles. The larger the number of possible nearest neighbours, the deeper the potential well, the smaller the mobility of the adparticles.

It can be concluded that the sticking coefficient as well as the mobility depend in such a way on the number of offered nearest neighbours that the perpendicular growth rate increases when the number of nearest neighbours offered to the adparticles increases.

As such, the difference in the number of nearest neighbours that these planes offer to the incoming adparticles causes an anisotropy in the perpendicular growth rate of the crystallographic planes. The plane that offers the lowest number of nearest neighbours to the incoming adparticles, will have the lowest perpendicular growth rate and thus forms also the resulting facets.

Beside the clearly faceted grains, it is also characteristic for zone Ic depositions that adparticles may overcome $E_{d, p h y s}$ or $E_{d, c h e m}$ but cannot diffuse off the grain or from one grain to another. Hence, the adparticles will not be able to overcome the barrier existing at the boundary of a grain or between two grains (see figure II.2.7.a). Therefore, no real interaction or competition between neighbouring grains sets in and straight, clearly faceted columns develop. Even in absence of an energetic bombardment, the voids between the columns will be filled up due to the increased adparticle mobility. As such, the columns are not separated by voids but only by grain boundaries. The resulting structure of a zone Ic deposition is shown in figure II.2.7.b.

Since the adparticles are not able to diffuse from one grain (column) to another, these grains (or columns) can only compete with each other due to shadowing. More precisely, a growth rate anisotropy only develops when there is an anisotropy in the flux of adparticles that a grain or column captures. This means that the tallest columns can be able to overgrow (or shadow) smaller columns. Since this growth competition is only based on geometry and shadowing but not on crystallographic reasons, no preferential out-of-plane orientation will develop in zone Ic structures.

(a)

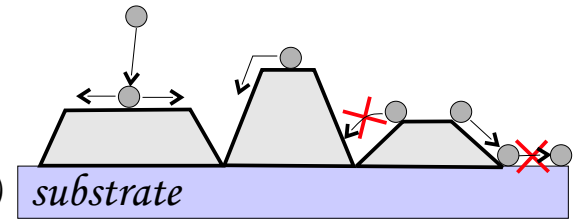

(b)

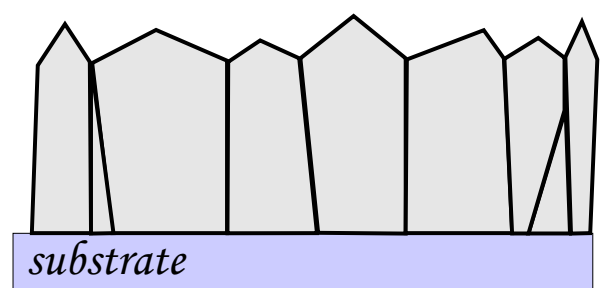

Figure II.2.7: Characteristic for zone Ic is that adparticles are able to diffuse around on a grain but are not able to diffuse from one grain to another (a), this gives rise to a faceted columnar structure (b).

Carrying out depositions in zone Ic conditions on a tilted substrate (tilt angle $\alpha$ ) may again lead to tilted columns (angle $\beta$ ). However, due to the increased mobility of the adparticles, the tangent-rule $(\tan \beta=1 / 2 \tan \alpha)$ is not anymore applicable. 


\section{Zone T:}

A logical sequence in further increasing the temperature $T$, is that the mobility of the adparticles is not anymore limited to one grain but that these adparticles are able to diffuse from one grain to another. This corresponds with the completion of the crystal growth but the prohibition of a re-structurative grain growth (both discussed in chapter I, §I.4). These different processes are shown in figure II.2.8.

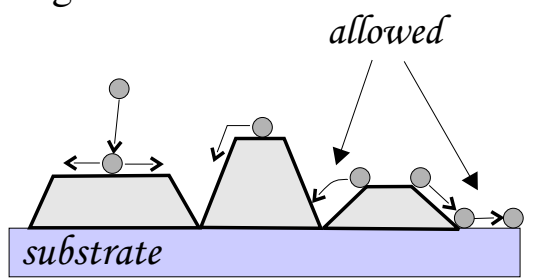

Figure II.2.8: Diffusion processes occurring in zone T conditions. Characteristic for zone T is that adparticles are now able to diffuse from one grain to another.

Since the mobility is high enough, the grains will again grow according to their most stable habit and again faceting will be observed, in which again the planes of lowest perpendicular growth rate form the resulting facets. Since no re-structurative grain growth can happen, a competitive growth of neighbouring grains should happen in an evolutionary way. However, next to geometric shadowing a second mechanism will enhance this overgrowth mechanism.

Assume initially random out-of-plane oriented and well faceted nuclei on a substrate, at conditions where the mobility of the adparticles is so high that they can diffuse from one nucleus to another. All these nuclei -and later on crystals and grains- are faceted by the same crystallographic planes of lowest perpendicular growth rate. Depositing during a specific time step causes these planes to increase with a layer of the same thickness, since all these planes have the same perpendicular growth rate. This proceeds as long as the several crystals don't touch or impinge each other, as is schematically shown in figure II.2.9.

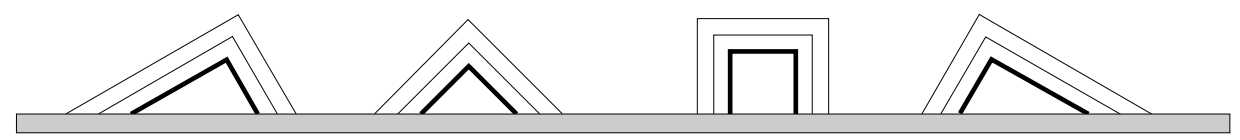

Figure II.2.9: Expansion of the facetted nuclei or crystals before impingement represented in a two dimensional way.

As soon as the impingement of the different crystals happens, a grain boundary is created. Further enlargement of these grains should take into account the immobility of that grain boundary as re-structurative grain growth is prohibited due to the low mobility. As schematically drawn in figure II.2.10, the immobility of that grain boundary and the identical growth rate of the facets, causes the overgrowth of one grain by another grain, since the adparticles are able to diffuse from one grain to another.

It is clearly seen in figure II.2.10 that the grain with the most tilted facet with respect to the substrate slowly envelops and overgrows the other grain.

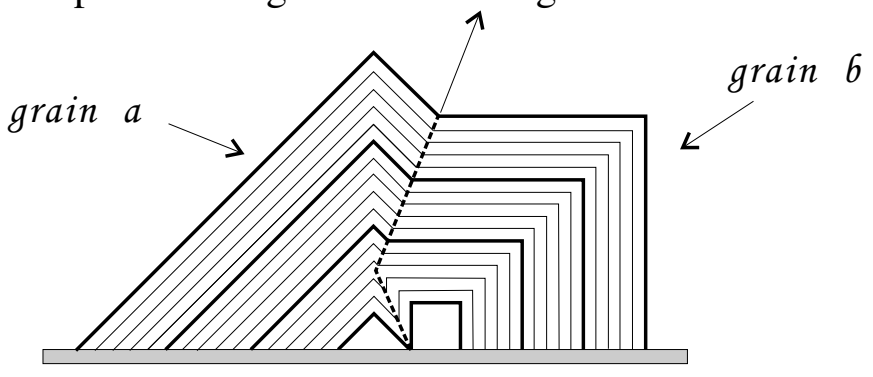

Figure II.2.10: Enlargement of the overgrowth process of faceted grains in which the grain with the most tilted facets (grain a) with respect to the substrate surface slowly envelops the other grain (grain b). 
Similar to van der Drift [30], representation of the further film growth according to the proposed growth shows the evolution to $\mathrm{V}$-shaped columns with a faceted surface, as shown in figure II.2.11.

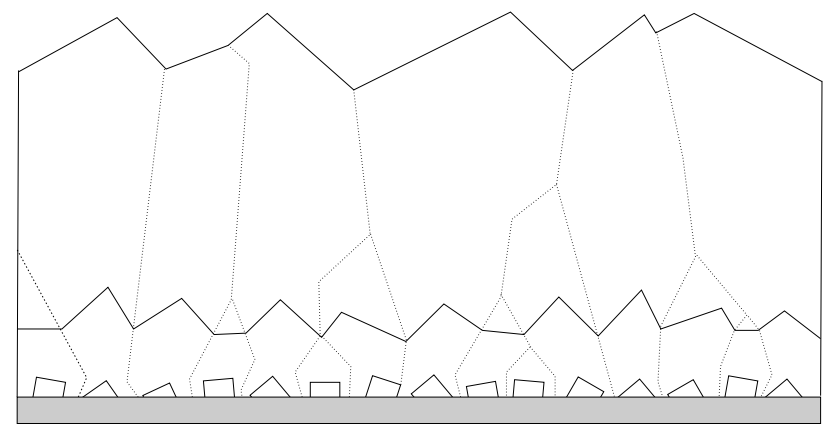

Figure II.2.11: Representation of a film grown in zone T conditions, resulting in faceted V-shaped columns according to van der Drift [30].

It can be concluded that the grains with the most tilted facets with respect to the substrate plane will envelop and overgrow the other grains. Comparing figure II.2.10 and figure II.2.4, it is clearly seen that these grains correspond to the grains with the geometric fastest growing direction perpendicular to the substrate. Hence, a mechanism in which the grains with geometric the fastest growing direction perpendicular to the substrate will overgrow all other grains is proposed. However, it is important to note that according to the presented model, an evolutionary overgrowth and hence the development of a clear out-of-plane alignment in zone $\mathrm{T}$ can only be obtained when the grains are well and unequivocally faceted.

In the above discussion, it was assumed that the adparticle mobility is high enough to allow the diffusion among several grains, i.e. zone T conditions. However, in case of a zone Ic deposition, i.e. when this diffusion from one grain to another grain is not possible, no evolutionary overgrowth will be observed. Since there is no interaction between the different grains, straight and perpendicular columns will establish in case of a deposition on a nontilted substrate. According to van der Drift ${ }^{\dagger}$, this results in a structure as shown in figure II.2.12. It is clearly seen that this structure corresponds with to the above discussed zone Ic structure since straight columns, without overgrowth but with faceted tops are observed. It is also observed that a random out-of-plane orientation is established since the initial orientation of the nuclei is conserved and because the facets point in all directions with respect to the substrate plane.

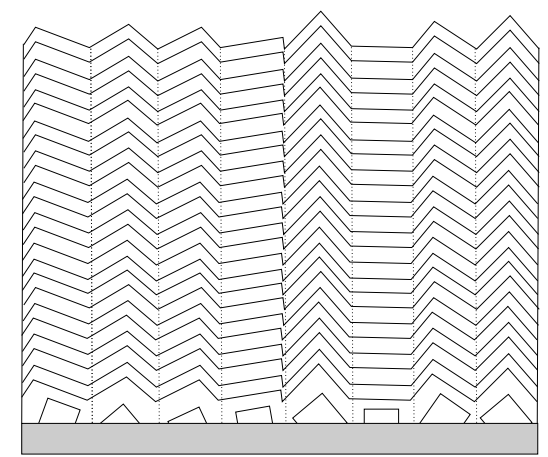

Figure II.2.12: Representation of the film growth in zone Ic conditions, resulting in faceted straight columns with random out-of-plane orientation.

\footnotetext{
${ }^{\dagger}$ It should be remarked that van der Drift obtained these results with the assumption of the total absence of adparticle mobility. However, this can't be right, since the total absence of adparticle mobility prohibits the nucleation of crystalline and faceted nuclei.
} 


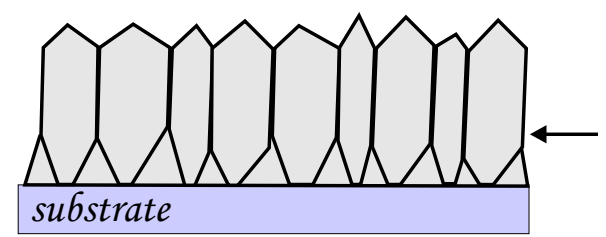

Figure II.2.13: Schematic drawing of a zone T structure, characterized by the V-shaped and faceted columns. The arrow indicates the film thickness at which the out-of-plane alignment is completed.

It can be concluded that in zone $\mathrm{T}$ depositions, different grains with random out-of-plane orientation will nucleate. Due to an anisotropy in growth rate -which depends on the crystallographic orientation- some grains will overgrow other grains. This results in an evolutionary selection of one crystallographic out-of-plane orientation. The out-of-plane alignment is completed at a certain film thickness which corresponds to the completion of the observed overgrowth, as marked in figure II.2.13. At higher film thickness, a columnar structure with faceted tops develops. It is important to note that the resulting preferential outof-plane orientation corresponds to the geometric fastest growing direction. This geometric fastest growing direction depends on the crystal habit which is formed by the crystallographic slowest growing planes.

Gilmer et al. [31,32] carried out atomistic Monte Carlo simulations in which zone T structures have been modelled.

\section{Zone II:}

Up to zone T temperatures, atoms are immobile as soon as they were incorporated in the growing film. However, when increasing the temperature $T$, already chemisorbed and thus stable atoms can become active. As discussed in chapter I ( $\$$ I.4 section re-structurative grain growth), this means that re-structurative grain growth will happen. More exactly, former stable nuclei or islands can become unstable and get incorporated into other islands by ripening, cluster diffusion or grain boundary migration. Since the unstable islands are dissolved in more stable islands, no evolutionary V-shaped overgrowth will be observed in the resulting film. Thus a columnar structure with straight columns penetrating through the whole film thickness will be formed, as shown in figure II.2.14.

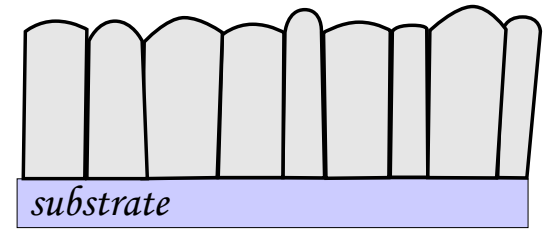

Figure II.2.14: Typical microstructure of a zone II film.

It was discussed that the re-structurative grain growth is not only driven by the minimization of the island or grain surface curvature but also by the minimization of the surface and interface energy (see chapter I, §I.4). Therefore, not each grain will aim for its own most stable habit but the whole film surface will do. Hence, the column tops will often not be perfectly faceted but only slightly curved (see chapter I, §I.5).

A second important consequence of the re-structurative grain growth and the corresponding minimization of the surface and interface energy is the development of a preferential out-ofplane orientation. As discussed in chapter I ( $\$$ I.3 section surface energy), the surface energy depends on the crystallographic plane. Hence, if the re-structurative grain growth drives the film to minimize the total surface energy, grains with a high energy plane parallel to the substrate will be consumed by grains with a low energy plane parallel to the substrate. Therefore, the resulting preferential out-of-plane orientation is the crystallographic orientation 
perpendicular to the plane of lowest surface energy. In case of TiN and YSZ, this corresponds with a preferential [100] and [111] out-of-plane orientation respectively [33,34].

Due to the nearly infinite surface diffusion and the appearance of the re-structurative growth, shadowing will have a minor influence on the growth. Hence, tilting the substrate with respect to the incoming material flux will not cause an inclination of the columns $\left(\beta=0^{\circ}\right)$.

Further increasing the temperature increases the effect of the re-structurative grain growth. Hence larger islands (grains) can be consumed by other grains, causing wider columns in the final film.

\section{Zone III:}

Further increasing the temperature $T$ not only allows surface diffusion and re-structurative grain growth, but also bulk diffusion. This implies that the resulting structure is not anymore related to the deposition process itself, because during -and even after- deposition grains in the internal structure of the film can nucleate, grow, shrink or even disappear. This grain growth in the bulk of the film has been extensively discussed by Thompson et al. [11,35,36]. Due to this bulk diffusion, the columnar structure is replaced by large crystalline grains. In case of thin films (up to several $\mu \mathrm{m}$ ) the grains can be much wider than the layer thickness. A schematic representation of such a zone III structure is shown in figure II.2.15.

It should be mentioned that this zone III can only exist if there is a mechanism which drives the grain boundaries to migrate and thus to form equiaxed grains in the internal film structure. Also something has to stop or block that grain boundary migration, since otherwise the film would again aim for its most stable situation, and thus become again a zone II structure. For sure, the presence of impurities can be a driving force for the grain boundary migration and the blocking of them. However, up to now it is not known if there exists still another mechanism -beside the impurities- making that the existence of zone III structure in as deposited pure films is not proved yet.

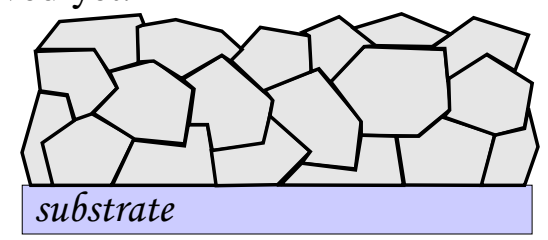

Figure II.2.15: Schematic representation of a zone III structure.

In case of a zone III growth, a preferential out-of-plane orientation can develop due to e.g. stress and strain minimization [37]. However, in most cases there is more than one mechanism responsible for the bulk diffusion and grain growth. Hence, there does not always develop a preferential orientation in zone III structures. As already mentioned, the resulting structure and crystallographic orientation are no longer related to the deposition process itself.

It can be noticed that no transition temperatures from one zone to another zone are mentioned in the above discussion of the BSZM. Although it can be indicative, it is not done since these transition temperatures will depend on the used class of materials [11]. Moreover, it should be obvious after the discussion of the BSZM that the transition from one zone to another merely depends on the offered mobility to the adparticles and to the growing film. Since this offered mobility not only results from the substrate heating, but also from e.g. a plasma-substrate interaction, it is not straightforward to mention exact transition temperatures.

\section{3: Revised structure zone model}

The former discussed BSZM describes the development of the microstructure and the corresponding crystallographic alignment in function of the overall mobility during the 
growth of pure materials. However, depositing pure materials without contamination of the growing film is in many cases not so evident. This contamination of the growing film can originate from a number of sources: (1) adsorption of impurities form the ambient (e.g. hydrocarbons, water vapours, $\left.\mathrm{CO}_{2}, \ldots\right)$ since the depositions are always carried out in a vacuum chamber with a finite base pressure, (2) bad cleaning of the substrate before starting the deposition, (3) impurities in the used gasses, (4) impurities in the material source. Therefore, the influence of these impurities and their possible influences on the elementary processes and phenomena of structure formation should be taken into account when describing a SZM. This has been extensively studied by Barna et al. [13-15, 18, 38-42] and will be described below as the revised structure zone model (RSZM).

It should be mentioned that the RSZM not only takes into account the influence of the above mentioned unintended impurities, but also the influence of intended co-deposited additives and dopants beyond their solubility limit and the influence of component(s) of multicomponent films at concentrations higher than the required stoichiometry [14]. Therefore, the expression 'impurity' will be used in a general meaning in the RSZM.

Impurities affect the surface processes of metallic adparticles directly in the following ways: - the impurity species can be adsorbed in various states and they can block the surface sites being active in binding metallic atoms or they can affect their surface diffusion.

- impurity adspecies can form complexes with the migrating or already bounded metallic adparticles. These complexes can have activation energies different from that of the metallic adparticles and can promote or hinder the basic structure forming phenomena.

- the impurities can accumulate and by this way they can develop their own two or three dimensional phase or those of their compounds. In such a case, impurity phases cause new local surface conditions both for the condensation of metallic adparticles and impurity adspecies.

- impurities can change the grain boundary mobility and the interface and surface energies. Summarizing, impurity species striking the surface can influence the nucleation, crystal growth or the re-structurative grain growth and thus the final film structure.

According to their effect on the structure evolution and the basic phenomena of structure formation, two types of impurities can be distinguished [38]. An impurity is called to be an inhibitor if it forms a two or three dimensional foreign phase on the free surface of the film or on the grain boundaries, thereby limiting the surface diffusion, the crystal growth and the restructurative grain growth. Its presence generally results in a smaller grain size. An impurity is called a promoter if its presence promotes the operation of diffusion or one of the basic structure forming phenomena, resulting in generally larger grains. One specific process of the incorporation of impurities during the film growth is their accumulation due to the monolayer growth on the surface of the film or on the grain boundaries. This process is called process induced segregation [39] and gives rise to a surface covering layer.

By carrying out vapour depositions of $\mathrm{Al}$ on amorphous carbon layers supported on a TEM microgrid, Barna et al. $[14,18]$ has shown that Sn and In can act as a promoter for the Al growth. This because it was observed that the grain size increased and that the grain shape rounded off when increasing the co-deposition flux of Sn or In. On the other hand, it was experimentally proved that $\mathrm{Pt}$ and $\mathrm{O}_{2}$ act as an inhibitor during the structure development of $\mathrm{Al}[15,40,41]$. 
The general influence of impurities as an inhibitor on the developing structure and the corresponding crystallographic alignment will be discussed on the basis of figure II.3.1 and has been described e.g. in [41].

\begin{tabular}{|c|c|c|c|c|}
\hline$I a+I b$ & \$ & \multicolumn{3}{|c|}{$I I I$} \\
\hline$I a+I b$ & Ic & $\tau$ & & $I I I$ \\
\hline$I a+I b$ & $I c$ & $\tau$ & $I I$ & $I I I$ \\
\hline$I a+I b$ & $I c$ & $\tau$ & $I I$ & III? \\
\hline
\end{tabular}

Figure II.3.1: Influence of the impurities on the different zones Ia, Ib, Ic, T, II and III of the SZM. (a) represents the BSZM, (b), (c), (d) the RSZM in case of a low, medium and high impurity concentration respectively.

As discussed in $\S I I .2$, the structure and the corresponding crystallographic alignment are described by the BSZM, at least in case that no impurities are involved during the thin film growth (see fig. II.3.1.a).

Therefore, at very low substrate temperatures (no thermal-induced mobility) a zone Ia or zone Ib structure will develop in the absence of impurities. Because the incoming metallic adparticles have no or nearly no mobility, the film growth proceeds in a hit-and-stick growth. Due to the absence of surface diffusion, the presence of impurities will hardly influence the film growth, since these impurities will also take part to the growth in a hit-and-stick way. Increasing the temperature, the surface mobility will increase. First, a zone Ic structure will develop in the absence of impurities. However, in case that the impurities are inhibitors, increasing the flux of these impurities will decrease the surface mobility and will thus retard the development of a zone Ic structure. Therefore, at low impurity flux, the transition from zone Ia or Ib to zone Ic will only occur at higher temperatures. At a still higher impurity concentration, it is possible that nearly all surface mobility is hindered and that no zone Ic structure will develop, but zone Ia or Ib.

According to the BSZM, further increasing the temperature gives rise to a zone T structure. Again, in the presence of an impurity flux, the surface mobility can be hindered. Special attention should be paid to the possible influence of the impurities on the development of the preferential crystallographic orientation. It was discussed in $\S$ II. 2 that in case of zone T depositions, the orientation with the largest geometric growth rate will finally become the preferential out-of-plane orientation due to an overgrowth mechanism. If there is an anisotropy in the surface chemical interaction of impurities with different crystallographic planes, the growth of certain crystallographic planes can be blocked at low or medium contamination level while other crystallographic planes can continue to grow. Therefore, the presence of impurities can lead to the development of special preferential out-of-plane orientations. Barna and Adamik [14, 15, 40] extensively investigated the example of $\mathrm{Al}+\mathrm{O}_{2}$. On the $\{111\}$ planes of $\mathrm{Al}$ the oxygen is segregated while on the $\{100\}$ and $\{110\}$ planes the oxygen is incorporated into the growing film. In this way, the $\{111\} \mathrm{Al}$ crystal planes become covered by an oxide impurity phase and their growth is blocked. In contrast, the $\{100\}$ and $\{110\}$ crystal faces grow further. This gives rise to a growth rate anisotropy and hence to an evolutionary overgrowth governed by the anisotropy of the oxygen segregation on the different crystallographic planes. At still higher impurity flux, the surface diffusion from one grain to another grain can be hindered, causing a transition from zone T to zone Ic. When the 
flux of impurities is too high, the growth of all crystals can be blocked and consequently even a zone Ia or Ib can appear since repeated nucleation may occur.

At temperatures where re-structurative grain growth sets in, a zone II structure will develop. As discussed in chapter I ( $\$$ I.4 section re-structurative grain growth), one of the driving forces for the re-structurative grain growth is the minimization of the surface- and interface energy. Hence, an increasing impurity flux can have again its influence on the resulting preferential orientation. At least if the presence of impurities causes another crystallographic plane to be the plane of lowest surface energy. Moreover, the presence of the impurities will again hinder the basic phenomena of structure formation. Hence, the developed zone II columnar structure can have a bimodal grain size distribution at a low impurity flux, due to the sometimes hindered re-structurative grain growth. Also the transition from zone T to zone II will be shifted to higher temperatures. At high impurity concentrations, zone II will even disappear from the SZM.

At very high temperatures, possibly a zone III structure could develop in the BSZM due to bulk diffusion and recrystallization. However, a driving force for that bulk diffusion is the presence of stress, irregularities in the film,... Hence impurities will enhance the appearance of bulk diffusion. Therefore, the zone III structure may occur at lower temperatures in the presence of impurities. At high impurity concentrations, all crystals will be covered by an impurity phase and a structure consisting globular or equiaxed grains will develop. Therefore, the difference between a zone Ia or Ib and a zone III structure will become less clear (see fig. II.3.1.d).

Adamik [15,40] and Barna [14] gave experimental evidence for the RSZM, by depositing thermal evaporated $\mathrm{Al}$ in the presence of an oxygen flow. As mentioned earlier, oxygen acts as an inhibitor for the growth development of Al. By increasing the oxygen flow, they could change the resulting structure from zone II to zone T towards finally to zone I (or zone III).

\section{4: Conclusions}

The resulting structure and the corresponding crystallographic out-of-plane alignment has been described in function of the increasing mobility offered to the growing film. In case that pure materials are deposited, the BSZM is valid. However, as soon as impurities are involved during the deposition, the RSZM has to be considered.

It could be concluded that the resulting structure is mainly determined by elementary processes such as substrate diffusion, self-surface diffusion, bulk diffusion and the fundamental phenomena of structure formation, i.e. nucleation, crystal growth and restructurative grain growth at the one hand and by the flux of impurities on the other hand. Since the resulting structure could be correlated by the elementary processes and phenomena of structure formation, also the resulting crystallographic out-of-plane alignment could be understood. Important to note here is that only in zone T and zone II depositions, the resulting preferential out-of-plane alignment is directly related to the used deposition conditions. In zone $\mathrm{T}$, the preferential orientation is a result of an evolutionary overgrowth due to a growth rate anisotropy of the different oriented grains, while in zone II the preferential orientation is a result of the aim for the lowest surface and interface energy of the total film.

As was experimentally demonstrated and extensively described by Barna et al., the influence of the impurities on the resulting structure and crystallographic orientation should always be kept in mind. However, it should be remarked that in some special cases some gasses should not be treated as an impurity source but as a real component of the resulting film. This is e.g. the case in reactive sputtering, where it is very often the intention to grow a metal oxide or 
metal nitride. If the reactive gas flow is high enough to ensure a completely stoichiometric oxide or nitride, all crystallographic grains are continuously covered by an oxide or nitride during the thin film growth. Hence, all depositions are carried out in the "high impurity flux" regime of the RSZM, if the reactive gas would be treated as an impurity. However, since the oxygen or nitrogen is a real component of the film and if the deposited film is a stoichiometric oxide or nitride, then the deposited material could be considered to be pure. Hence the BSZM is valid, at least when the deposition is not influenced by other impurities such as water vapour, hydrocarbons, $\mathrm{CO}_{2}, \ldots$

It should be mentioned that when applying the BSZM the homologous temperature $T$ should be calculated from the substrate temperature $T_{s}$ and the melting temperature $T_{m}$ of the metal oxide or nitride (and not the melting point of the pure metal).

\section{References}

1: A.M. Brown, M.F. Ashby: Acta Metall. 28 (1980) p.1085-1101

2: B.A. Movchan, A.V. Demchishin: Phys. Met. Metallogr. 28 (1969) p.83-89

3: J.V. Sanders chapter I "Structure of Evaporated Metal Films" in "Chemisorption and

Reactions on Metallic Films" published by Academic Press ISBN: 0120580012

4: C.R.M. Grovenor, H.T.G. Hentzell, D.A. Smith: Acta Metall. 32 (1984) p.773-781

5: H.T.G. Hentzell, C.R.M. Grovenor, D.A. Smith: J. Vac. Sci. Technol. A2(2) (1984) p.218219

6: R. Messier, A.P. Giri, R.A. Roy: J.Vac. Sci. Technol. A2(2) (1984) p.500-503

7: J.A. Thornton: J. Vac. Sci. Technol. 11(4) (1974) p.666-670

8: J.A. Thornton: J. Vac. Sci. Technol. 12(4) (1975) p.830-835

9: J.A. Thornton: Ann. Rev. Mater. Sci. 7 (1977) p.239-260

10: J.A. Thornton: J. Vac. Sci. Technol. A4(6) (1986) p.3059-3065

11: C.V. Thompson: Ann. Rev. Mater. Sci. 30 (2000) p.159-190

12: E. Mirica, G. Kowach, H.Du: Cryst. Growth \& Des. 4(1) (2004) p.157-159

13: P.B. Barna, M. Adamik in "Science and Technology of thin films" published by World scientific ISBN: 9810221932

14: P.B. Barna, M. Adamik: Thin Solid Films 317 (1998) p.27-33

15: M. Adamik, P.B. Barna, I. Tomov: Thin Solid Films 317 (1998) p.64-68

16: P.J. Kelly, R.D. Arnell: J. Vac. Sci. Technol. A16 (5) (1998) p.2858-2869

17: Y. Kajikawa, S. Noda, H.Komiyama: J. Vac. Sci. Technol. A21 (6) (2003) p.1943-1954

18: I. Petrov, P.B.Barna, L. Hultman, J.E. Greene: J. Vac. Sci. Technol. A21 (5) (2003) S117S128

19: A.L. Barabási, H.E. Stanley in "Fractal concepts in surface growth" published by

Cambridge University Press ISBN:0 521483182

20: D.L. Smith in "Thin film deposition: principles \& practice" published by McGraw-Hill ISBN: 0070585024

21: K.H. Müller: J. Appl. Phys. 62 (5) (1987) p.1796-1799

22: K.H. Müller: J. Appl. Phys. 58 (7) (1985) p.2573-2576

23: K.H. Müller: J. Vac. Sci. Technol. A6 (3) (1988) p.1690

24: H.J. Leamy, A.G. Dirks: J. Appl. Phys. 49(6) (1978) p.3430-3438

25: G.H. Gilmer in "Handbook of crystal growth" Vol. 1, part a. Chapter 8, published by Elsevier Science ISBN: 044889086

26: L. Abelmann, C. Lodder: Thin Solid Films 305 (1997) p.1-21

27: K.H. Müller: Phys. Rev. B 35 (1987) p.7906-7913

28: P. Hartman, W.G. Perdok: Acta Cryst. 8 (1955) p.49-52 and p.521-529

29: H. Huang, G.H. Gilmer: J. Appl. Phys. 84 (1998) p.3636-3649 
30: A. van der Drift: Philips Res. Repts 22 (1967) p.267-288

31: G.H. Gilmer, H. Huang, T. Diaz de la Rubia, J. Dalla Torre, F. Baumann: Thin Solid Films 365 (2000) p.189-200

32: J. Emiliano Rubio, M. Jaraiz, I. Martin-Bragado, J.M. Hernandeze-Mangas, J. Barbolla, G.H. Gilmer : J. Appl. Phys. 94(1) (2003) p.163-168

33: J.P. Zhao, X. Wang, Z.Y. Chen, S.Q. Yang, T.S. Shi, X.H. Liu: J. Phys. D: Appl. Phys. 30 (1997) p.5-12

34:A. Christensen, E.A. Carter: Phys. Rev. B 58(12) (1998) p.8050-8064

35: C.V. Thompson, R. Carel: Mater. Sci. Eng. B32(1995) p.211-219

36: C.V. Thompson, R. Carel: J. Mech. Phys. Solids. 44(5) (1996) p.657-673

37: J.-M. Zhang, K.-W. Xu, V.Ji: Appl. Surf. Sci. 185 (2002) p.177-182

38: M. Adamik: Ph.D. "Texture evolution in polycrystalline thin films" (2000) ISBN 963 7371168

39: P.B. Barna in "Crystal growth and recrystallisation during structure evolution of thin films" p.295-309 in "Diagnostics and application of thin films" published by Inst. Phys. Publ. (1992) ISBN: 0750301651

40: M. Adamik, P.B. Barna, I. Tomov, D. Biro: Phys. Stat. Sol. (a) 145 (1994) p.275-281

41: P.B. Barna: Proc. IX IVC-V ICSS, Madrid (1983) p.382-396

42: P.B. Barna, M. Adamik in "Protective coatings and thin films" published by Kluwer Academic, Nato Series (1997) ISBN 0792343808 


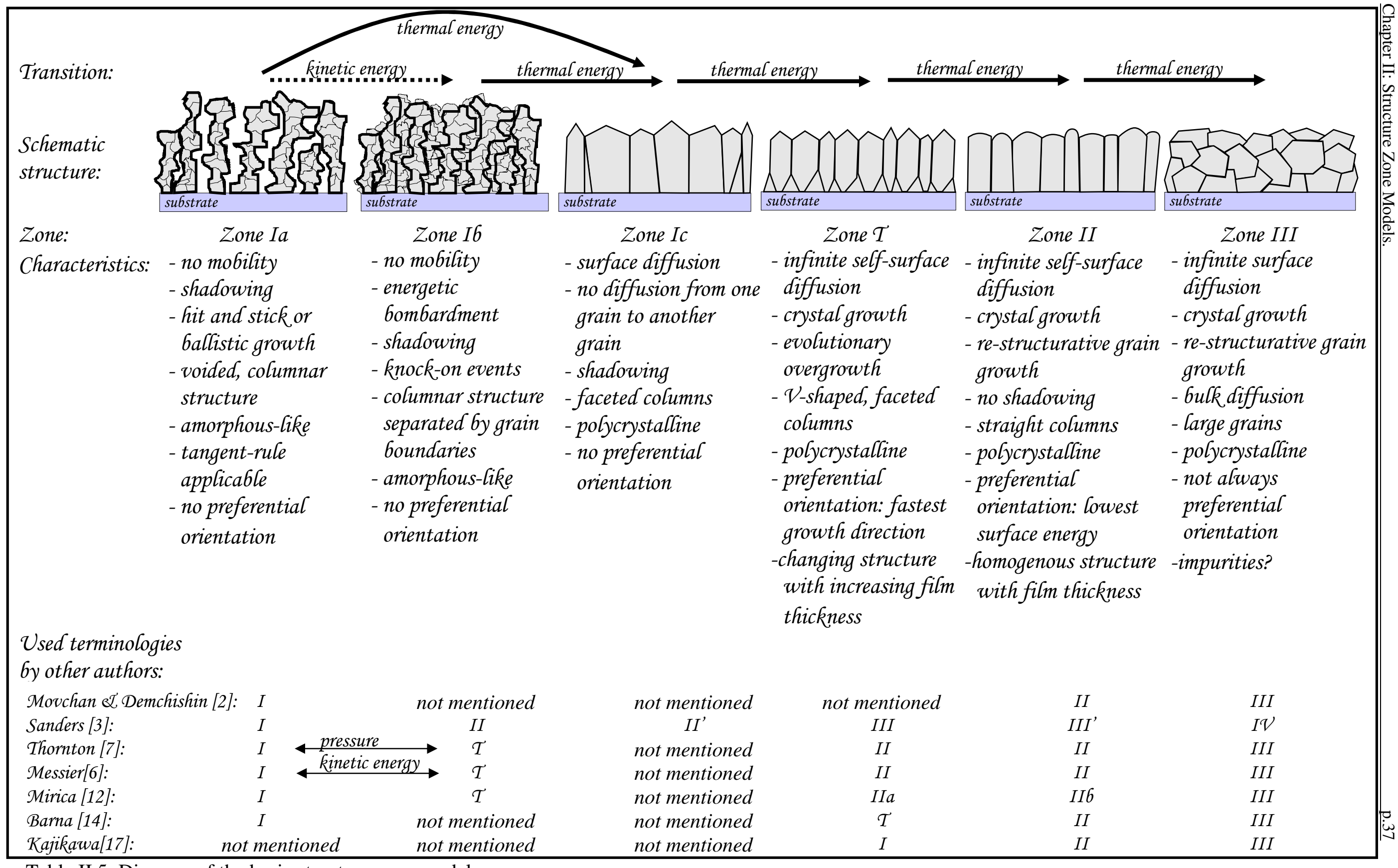

Table II.5: Diagram of the basic structure zone model. 


\section{Chapter III: Reactive unbalanced magnetron sputter deposition.}

\section{1: Introduction}

Evidently, to investigate the growth mechanism of biaxially aligned thin films, these thin films have to be grown using a certain deposition technique. Hence, also the processes occurring at the deposition source and during the transport from the source to the substrate have to be understood. Although the number of existing deposition techniques is enormous, all thin films in this thesis were deposited by reactive unbalanced magnetron sputtering. This chapter is an attempt to introduce the reader into the "sputter guild" as my promoter introduced me into it some years ago.

\section{2: Sputtering: ion-solid interaction}

When an energetic particle (atom or ion) hits a solid surface, atoms, molecules or even clusters may be ejected. This phenomenon is known as sputtering. Hence, solid surfaces are eroded under particle bombardment. This erosion is usually characterized by the sputter yield $Y$ which is defined as the mean number of emitted atoms per incident particle. The bombarding particle dissipates its energy by nuclear collisions with target atoms. If these recoiled target atoms are energetic enough, they collide with other atoms; etc. If target atoms in the vicinity of the surface receive enough momentum and move in the appropriate direction to surpass the surface barrier, they may leave the target and are said to be sputtered [1-5]. Sigmund [1] and Thompson [2] provided a theoretical description of the total sputter yield, and the energy and angular distribution of the sputtered atoms respectively. This theoretical description will be briefly discussed in the following section. This because the sputter process, and more specific the energy and angular distribution of the sputtered particles, and the transport of these sputtered particles from the target to the substrate are needed in chapter VII, in order to find a correlation between some deposition parameters and the experimentally obtained crystallographic alignment.

According to Sigmund, it is convenient to distinguish the collision cascade into three regimes: the single-knockon regime, the linear cascade regime and the spike regime [1].

In the single-knockon regime, the bombarding particle transfers energy to target atoms which, possibly after having undergone a small number of one-to-one collisions, are ejected from the surface. In the linear and the spike regime, the recoiled target atoms are energetic enough to generate secondary and higher-generation recoils. In this way, a cascade of recoils is generated, and again some atoms at the surface may by ejected from the solid. In the linear regime, the density of recoils is sufficiently low to ensure that most collisions involve one moving and one stationary particle. This regime is called linear since the sputter yield is found to be proportional to the first power of the projectile energy. In the spike regime, the density of recoils is so high that collisions between two moving atoms are frequently occurring. A schematic drawing of the occurring collisions in these three regimes is shown in figure III.2.1. Quantitatively, the single-knockon regime falls into the lower and medium incident energy region, except for light ions where, because of inefficient energy transfer, it extends up to the lower keV region. The linear cascade regime is characteristic for bombarding particles with an energy from several hundreds $\mathrm{eV}$ to some $\mathrm{keV}$ and $\mathrm{MeV}$, except for the heavier ions, which tend to cause the spike regime. As will be discussed in section III.3, in case of magnetron sputtering the bombarding particles have a energy in the range of several hundreds $\mathrm{eV}$. Therefore, the linear regime is of main interest. 

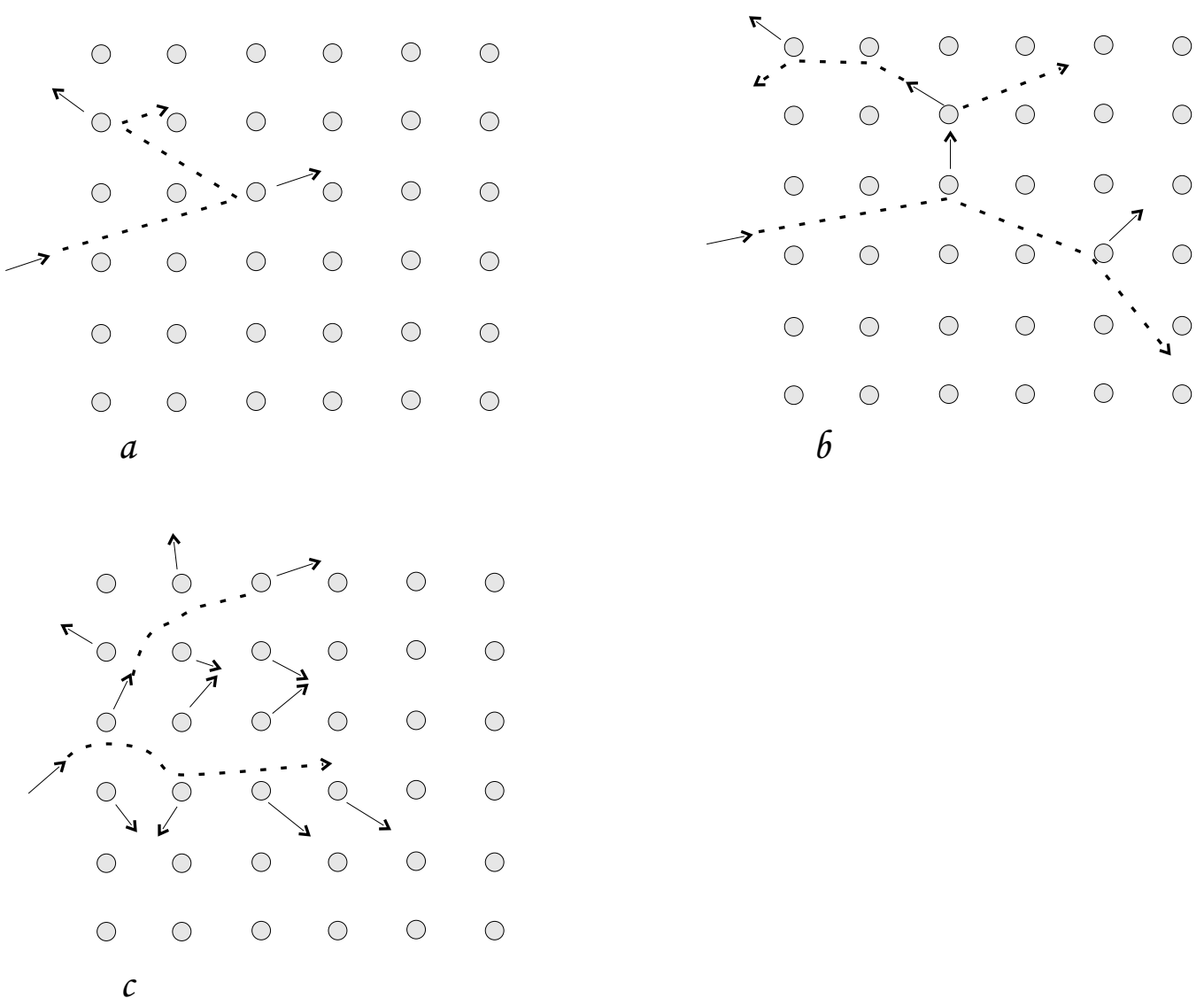

Figure III.2.1: Three regimes of collision cascades: the single-knockon regime, in which recoiled atoms are only able to recoil one other atom (a), the linear cascade regime in which recoiled atoms are able to generate a cascade of collisions and recoiled atoms. The density of recoils is low enough to ensure that all collisions are between one moving and one stationary atom (b), and spike regime in which collisions between two moving atoms are allowed (c).

\section{Sputter yield:}

As already stated, the sputter yield $Y$ is defined as the number of sputtered atoms per bombarding particle. To calculate the sputter yield in function of the energy of the bombarding particle, it should be calculated how much atoms suffer a recoil and how many of these recoiled atoms are able to escape from the surface.

Let $n\left(E, E_{0}\right)$ be the mean number of atoms set in motion with an initial energy larger than some value $E_{0}$ in a cascade initiated by a primary particle of initial energy $E\left(>E_{0}\right)$. Allowing for elastic collisions only, and ignoring all binding forces between target atoms, one finds the expression [6]:

$$
n\left(E, E_{0}\right)=\Gamma_{m} \frac{E}{E_{0}} \text { for large E (eq.III.2.1). }
$$

With $\Gamma_{m}$ a parameter only depending on the index $m$, which is a parameter depending on the energy $\mathrm{E}$ and which characterizes the scattering cross section. Values for $\Gamma_{m}$ and $m$ are given in table III.2.2.

Table III.2.2: some values for $m$ and $\Gamma_{m}$.

\begin{tabular}{|l|l|l|l|l|}
\hline$m$ & 0.500 & 0.333 & 0.250 & 0.000 \\
\hline$\Gamma_{m}$ & 0.361 & 0.452 & 0.491 & 0.608 \\
\hline
\end{tabular}


However, a particle bombarding a solid will not only dissipate its energy by elastic nuclear collisions, but also by exciting the electrons. This energy delivered to the electrons will not provide a chance to the atoms to recoil, since this energy will be immediately shared with all other electrons. Therefore, the total number of atoms set in motion after the impact of a primary particle with energy $E$, will not be $n\left(E, E_{0}\right)$ but

$$
n\left(E, E_{0}\right) \frac{F_{D}(E, \boldsymbol{\Omega}, \boldsymbol{r})}{E} \text { (eq.III.2.2) }
$$

in which $F_{D}(E, \boldsymbol{\Omega}, \boldsymbol{r})$ is the density of deposited energy for a particle of energy $E$ impinging under direction $\boldsymbol{\Omega}$ at the solid surface at position $\boldsymbol{r}$, slowed down due to the continuous drain of energy to the electrons.

Therefore, the mean number of atoms moving at any time with an energy $\left(E_{0}, d E_{0}\right)$ is given by

$$
n\left(E, E_{0}\right) \frac{F_{D}(E, \boldsymbol{\Omega}, \boldsymbol{r})}{E} d t_{0}=n\left(E, E_{0}\right) \frac{F_{D}(E, \boldsymbol{\Omega}, \boldsymbol{r})}{E} \frac{d E_{0}}{v_{0}\left|d E_{0} / d x\right|} \text { (eq. III.2.3) }
$$

in which $d t_{0}$ is the mean time needed by a recoil atom to slow down from $E_{0}+d E_{0}$ to $E_{0}, v_{o}$ is the velocity of a target atom with energy $E_{0}$.

Hence, the impact of an energetic particle generates a stationary distribution of moving target atoms. Assuming that the direction in which these target atoms move is isotropic, it can be calculated how much of these target atoms are able to reach the surface under a specific direction $\boldsymbol{\Omega}_{0}$. This, together with eq. III.2.1 gives an expression for the mean number of atoms moving with an energy $\left(E_{0}, d E_{0}\right)$ in the direction $\left(\boldsymbol{\Omega}_{0}, d^{2} \Omega_{0}\right)$ in the volume $\left(\boldsymbol{r}, d^{3} r\right)$ :

$$
\Gamma_{m} \frac{F_{D}(E, \boldsymbol{\Omega}, \boldsymbol{r})}{E_{0}} \frac{d E_{0}}{v_{0}\left|d E_{0} / d x\right|} \boldsymbol{\Omega}_{\boldsymbol{o}} \frac{d^{2} \Omega_{0}}{4 \pi} \text { (eq.III.2.4) }
$$

Multiplication with $v_{0}$ finally yields the current density of atoms reaching the target surface. If these particles can overcome the potential barrier existing at the surface, they are considered as sputtered. The simplest model for surface binding is based on a planar surface barrier $U_{s}$. In that case, the probability $\mathrm{P}\left(E_{0}, \theta_{0}\right)$ to be sputtered is given by:

$$
\begin{aligned}
& \mathrm{P}\left(E_{0}, \theta_{0}\right)=1 \text { if } E_{0, x}>U_{\mathrm{s}} \\
& \mathrm{P}\left(E_{0}, \theta_{0}\right)=0 \text { if } E_{0, x}<U_{\mathrm{s}}
\end{aligned}
$$

In which $E_{0, x}$ expresses the energy of the recoiled target atom in the direction perpendicular to the target surface.

Finally, an expression for the sputter yield of a particle impinging with an energy $E$ and angle $\theta$ at the surface in function of the energy $E_{0}$ of the sputtered target atoms $(\mathrm{r}=0)$ and their angular direction $\theta_{0}$ is given:

$$
d^{3} Y=\Gamma_{m} \frac{F_{D}(E, \theta, 0)}{E_{0}} \frac{d E_{0}}{\left|d E_{0} / d x\right|} \cos \theta_{0} \frac{d^{2} \Omega_{0}}{4 \pi} \text { (eq.III.2.5) }
$$

Sigmund simplified this expression in case for low energetic $(E<1 \mathrm{keV})$ and perpendicular $\left(\theta=0^{\circ}\right)$ bombardment to a total yield:

$$
Y(E)=\frac{3}{4 \pi^{2}} \alpha \frac{\gamma E}{U_{s}} \text { (eq.III.2.6) }
$$

in which $\gamma$ is the energy transfer mass factor, $U_{s}$ is the surface binding energy and $\alpha$ is dimensionless function of the angle of incidence $\theta$, the mass ratio between the incoming particle and the target atom and the primary energy $E[6]$.

It is observed that the sputter yield $Y$ is indeed linear with the first power of the energy $E$.

Beside the analytical theory developed by Sigmund, a lot of computer simulations are developed in order to follow up the trajectory of the incident projectile, the evolution of the 
cascades in the target and hence the sputter mechanism. There exist Monte Carlo- binary collision codes (e.g. SRIM [7], TRIM [8], MARLOWE [9]) and molecular dynamics codes (e.g. the work of Harrison [10]).

It should be remarked that the above discussed analytical theory is only valid in case that the target is amorphous. Large differences can be obtained when the target is monocrystalline $[11,12]$.

\section{Energy and angular distribution:}

Nice about the analytical theory is that the energy and angular distribution of the sputtered atoms can be immediately deduced from eq.III.2.5.

Assuming a planar surface barrier $U_{s}$ that the recoiled target atoms have to overcome before being sputtered and taking into account the conservation of momentum, the following equations must be fulfilled:

$$
\begin{aligned}
& E_{1} \cos ^{2} \theta_{1}=E_{0} \cos ^{2} \theta_{0}-U_{s} \text { (eq. III.2.7.a) } \\
& E_{1} \sin ^{2} \theta_{1}=E_{0} \sin ^{2} \theta_{0} \quad \text { (eq. III.2.7.b) }
\end{aligned}
$$

in which the subscript 0 indicates the situation before the target atom reaches the surface and the subscript 1 the situation after the atom leaves the target, as also shown in figure III.2.3. $E$ indicates the energy, $v$ indicates the velocity and $\theta$ the direction with respect to the surface normal.

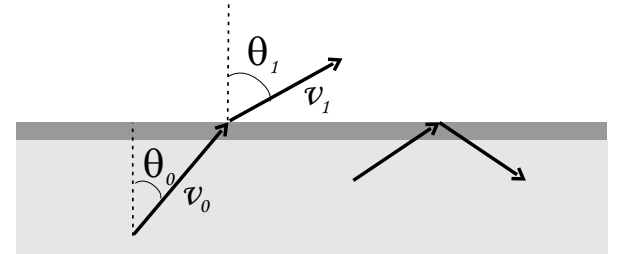

Figure III.2.3: Effect when an atom passes the planar surface barrier $U_{s}$ (left) or is reflected at the target surface (right). The indices 0 and 1 indicate the situation just before and just after the ejection respectively.

It results from eq. III.2.7.a that $E_{1} \cos \theta_{1} d^{2} \Omega_{1}=E_{0} \cos \theta_{0} d^{2} \Omega_{0}$ and hence, eq.III.2.5 yields:

$$
d^{3} Y=\Gamma_{m} \frac{F_{D}(E, \theta, 0)}{\left(E_{1}+U_{s}\right)^{2}} \frac{E_{1} d E_{1}}{\left|d E_{0} / d x\right|_{E_{0}=E_{1}+U_{s}}} \cos \theta_{1} \frac{d^{2} \Omega_{1}}{4 \pi} \text { (eq.III.2.8) }
$$

The factor $d E_{0} / d x$ expresses the loss in energy when a target atom moves through a medium. Hence $d E_{0} / d x$ is given by $N S_{n}(E)$ in which $N$ is the density of the medium and $S_{n}(E)$ is the nuclear stopping cross section. According to Sigmund [6] the nuclear stopping cross section is proportional to $E^{1-2 m}$, with $\mathrm{m}$ the same parameter as in table III.2.2. This parameter $m$ depends on the energy $E$ and is 0 for $E=U_{s}, 0.2$ à 0.3 for $E=1 \mathrm{keV}$ and reaches unity when $E$ is very high. Hence eq.III.2.8. gives an expression for the energy and angular distribution of the sputtered atoms:

$$
\frac{d^{3} Y}{d E_{1} d^{2} \Omega_{1}} \approx \frac{E_{1}}{\left(E_{1}+U_{s}\right)^{3-2 m}} \cos \theta_{1} \text { (eq.III.2.9) }
$$

The energy distribution $N(E) \approx \frac{E_{1}}{\left(E_{1}+U_{s}\right)^{3-2 m}}$ has its maximum at $N_{\max }=\frac{U_{s}}{2(1-m)}$. The angular distribution of the sputtered particles seems to have a cosinus-like behavior. Thompson [2] derived this expression in case for very low energetic bombardment $(m=0)$, leading to the well known energy distribution function:

$$
N(E) \approx \frac{E_{1}}{\left(E_{1}+U_{s}\right)^{3}} \text { and } N_{\max }=\frac{U_{s}}{2} \text { (eq.III.2.10). }
$$


It should be remarked that eq.III.2.7 only takes into account that an atom is refracted upon passing through the planar surface barrier. However, it was not taken into account that some atoms (those with $E_{0, x}<U_{s}$ ) are really hindered to be sputtered from the target due to the existence of the surface barrier. Secondly, it is noticed in eq.III.2.9 that this analytical model suggests that the energy distribution and the angular distribution are independent of each other.

As already mentioned, the effect of the particle bombardment, the collision cascade and the sputtering process has been simulated by several models. Figure III.2.4 shows the energy distribution of $\mathrm{Cu}$ sputtered by $400 \mathrm{eV}$ Ar as calculated by the analytical model and as simulated by SRIM [7]. It is noticed that the high energy tails are corresponding very well, while the distribution shows a shift of $3.52 \mathrm{eV}$ in the low energy region, which is exactly the height of the surface barrier for $\mathrm{Cu}$. This shift results from the omission of the hindering effect of the surface barrier in the analytical model.

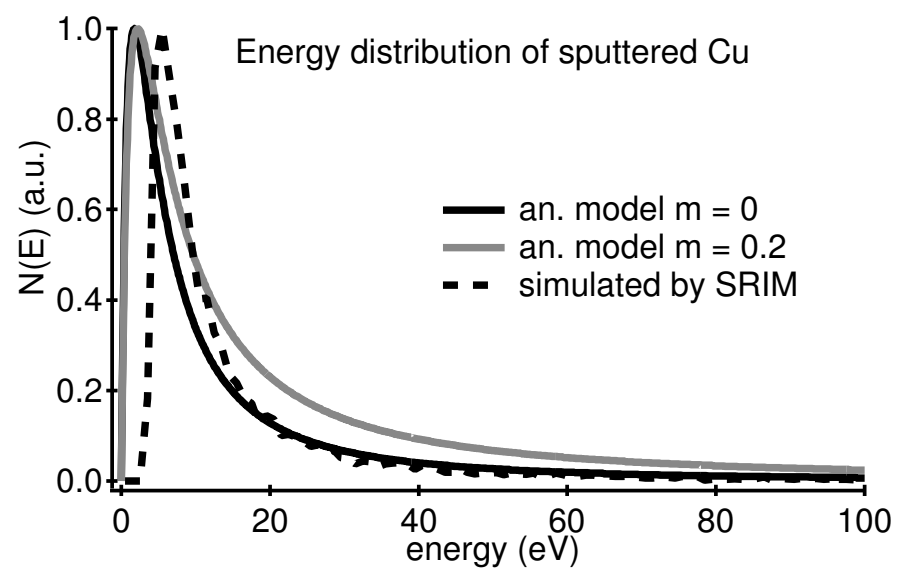

Figure III.2.4: The energy distribution of $\mathrm{Cu}$ sputtered by $400 \mathrm{eV}$ Ar. The surface barrier $U_{s}$ for $\mathrm{Cu}$ was set at $3.52 \mathrm{eV}$. The full lines are the distributions as calculated by the analytical model, while the dashed line is the distribution as simulated by SRIM.

Figure III.2.5. shows the angular distribution of $\mathrm{Cu}$ sputtered by $400 \mathrm{eV}$ Ar as calculated by the analytical model (cosinus behaviour) and as simulated by TRIM [8]. Although these distributions seem to correspond rather well, it will be discussed in chapter VII that the angular distribution may not be treated as energy independent.

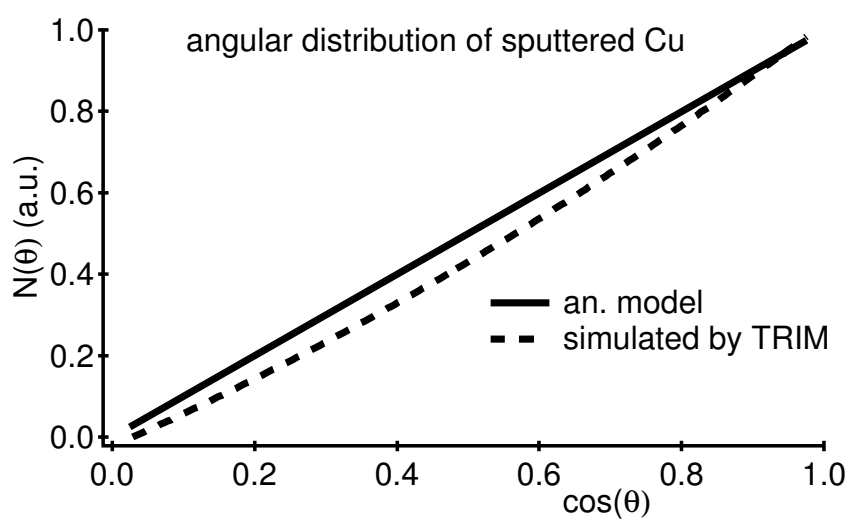

Figure III.2.5: The angular distribution of $\mathrm{Cu}$ sputtered by $400 \mathrm{eV}$ Ar. The surface barrier $U_{s}$ for $\mathrm{Cu}$ was set at $3.52 \mathrm{eV}$. The full line is the distribution as calculated by the analytical model, while the dashed line is the distribution as simulated by TRIM. 


\section{3: Basics of magnetron sputtering}

In the above section, the erosion of a solid by particle bombardment was discussed. If this effect is to be used as a deposition mechanism, a controllable flux of bombarding particles on the target should be provided.

A possible way to obtain a continuous bombardment, is by applying a negative potential to a target (with respect to some anode) and by igniting a glow discharge which creates a plasma in front of the target.

A plasma is a partially ionized gas consisting of more or less equal numbers of positively and negatively charged particles, and a different number of neutral molecules. It can be assumed that the charged particles are electrons and singly charged positive ions. Therefore, the ionization degree or plasma density is defined as the number of electrons $n_{e}$, divided by the sum of electrons and neutrals $\left(n_{e}+n\right)$. In the type of plasmas used in magnetron sputtering, the ionization degree is in the order of $10^{-3}$ à $10^{-4}[13,14]$. Although the Coulomb interaction between charged particles is strong and long-range, it is possible to assume for an undisturbed plasma that the charged particles move around as free particles, since the sum of all interactions tends to cancel out. Due to their much lower mass, the electrons have an enormous velocity compared to the ions and neutrals. If a spherical plasma were created, both types of particles (positive and negative) would diffuse outward, but the electrons would diffuse faster because of their much higher velocity. As the electrons moves away from the centre of the sphere, the net charge within the sphere becomes positive, exerting an attractive force on the electrons. Hence, a stable plasma has a small electric field at the boundary so that electrons and positive ions diffuse at the same rate. This potential difference is approximately equal to the average electron energy, usually a few $\mathrm{eV}$. Thus, a plasma is a region of equipotential with a small potential gradient at its boundary, such that the bulk of the plasma is at a small, more positive potential. This potential is called the plasma potential $V_{p}$. The enormous velocity of the electrons not only forces the plasma to reach the plasma potential $V_{p}$, but also forces an electrically floating object located in the plasma to reach a negative potential. If a floating object is inserted into the plasma, the initial flux of electrons to this object will be much higher than the flux of positive ions due to the difference in mass. Therefore, this object will get negatively biased, repelling the electrons. The potential of the object is in equilibrium when it is high enough to repel that much electrons to equal the ion and electron flux. This equilibrium potential is called the floating potential $V_{f}$ and is usually 5 à $15 \mathrm{eV}$ lower than the plasma potential, depending on the average energy of the electrons. The disturbance of the plasma by the insertion of a floating or biased object causes a local inhomogeneity of the plasma density. This because the electrons are repelled or attracted by the object, and hence the electron density is locally lower or higher. The region where the electron density is inhomogeneous is called the sheath.

Finally, when an object at large negative potential is inserted into the plasma, the potential varies as shown in figure III.3.1.

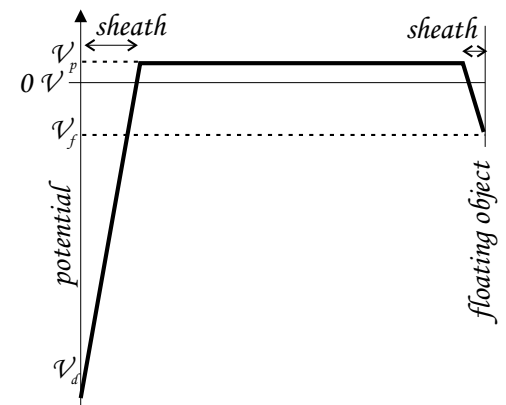

Figure III.3.1: Variation of the potential in a plasma when a negatively biased and floating object are inserted. The sheath is the region where the plasma density and the potential are not constant in space. 
In fact, this is the setup used to create a glow discharge [15-18]. When a glow discharge is used as a deposition method, the target acts as the cathode. If the potential difference between the negatively biased object (cathode) and plasma potential is large enough, some electrons will be accelerated that much that they receive enough energy to ionize neutral gas atoms. If this ionization takes place in the sheath, the ions are accelerated back towards the cathode and when energetic enough they may sputter the cathode, as described in section III.2. However, the energetic particle bombardment will not only generate sputtered cathode atoms, but also secondary electrons. The number of secondary electrons emitted by the cathode per incident ion is called the ion induced secondary electron (ISEE) coefficient $\gamma_{i}$, and depends on the cathode material and the energy and kind of the bombarding particle. These generated secondary electrons are again accelerated away from the cathode and may reach again enough energy to ionize neutrals.

The discharge current $I_{d}$ is the summation of the ion current $I_{i}$ towards the cathode and the electron current $I_{e}$ away from the cathode: $I_{d}=I_{i}+I_{e}=I_{i}\left(1+\gamma_{i}\right)$. In case of the bombardment of $\mathrm{Ar}^{+}$ions of a few hundred $\mathrm{eV}$ on a metallic cathode, the ISEE coefficient $\gamma_{i}$ is in the order of $0.1[15,17]$. The discharge current is self-sustaining when one impinging ion is energetic enough to create enough secondary electrons to ensure again one ionisation in the sheath. In order to optimize the bombardment flux on the target, and hence the ionisation just in front of it, magnets are often placed just behind the target. These magnets causes the secondary electrons to be captured just in front of the target, and hence to locally enlarge the ionisation degree and the bombardment flux. This idea is used in a magnetron sputter source, of which an example is shown in figure III.3.2.

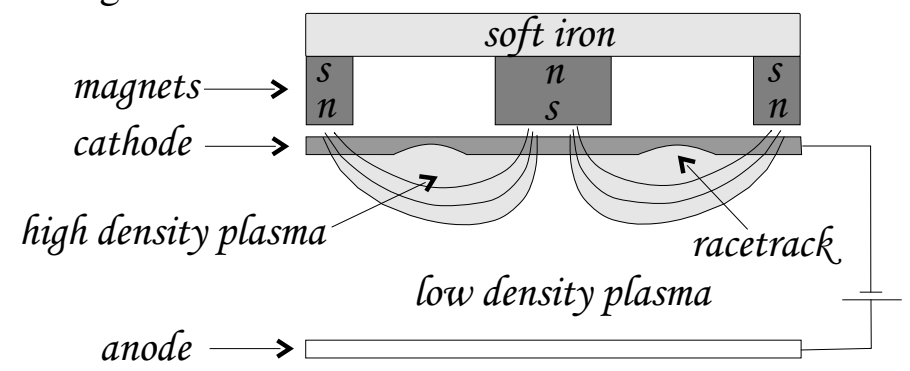

Figure III.3.2: Sketch of a planar circular magnetron. These magnets are placed just behind the target. The magnetic field causes the electrons to move in orbits just above the target, increasing the ionisation degree locally, giving rise to a racetrack. Further away from the target, a plasma of lower density is present.

In case of a planar circular magnetron, two magnet rings (with different diameter) are placed behind the target. This causes the secondary electrons to move in orbits, as shown in figure III.3.3. The secondary electrons are accelerated away from the target by the electric field. If a secondary electron does not collide with another particle, it conserves its energy and may reach again the target surface where it is possibly re-captured. Otherwise, it is reflected at the target surface and starts again another orbit, causing electrons running around on the target as shown in figure III.3.3. If an electron collides and ionizes a neutral, it looses some energy. When an electron lost nearly all its energy, it may diffuse away from the target causing a lower plasma density up to the chamber walls. Due to the specific magnetic field shape, the ionisation degree is locally increased and hence the erosion rate from the target will not be uniform. Therefore, there develops a local increased erosion, which is often called a racetrack since it originates from the electrons running above the target in a rotary motion. 


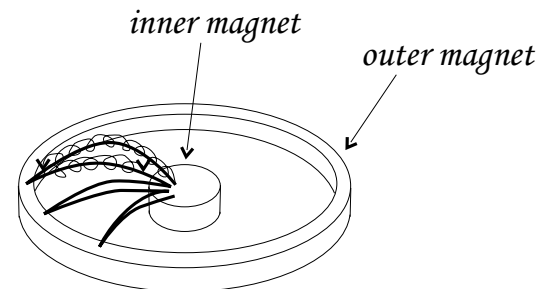

Figure III.3.3: Schematic drawing of the magnet array and electron motion in a planar circular magnetron.

It was described by Buyle et al.[19] how the discharge potential $V_{d}$ in case of planar circular magnetron sputtering can be calculated. As already mentioned, the number of electrons escaping from the target after an ion impact is given by $\gamma_{i}$. However, some of these electrons may be recaptured in the target before ionizing gas atoms. The fraction of non-recaptured electrons is expressed by $f$. Hence $\gamma_{i} f$ electrons really escape from the target and are accelerated in the sheath, gaining enough energy to ionize gas atoms. The mean energy lost when ionizing a gas atom is given by $W$. However, it should be taken into account that an electron may ionize a gas atom, i.e. creating an electron-ion pair at a height $z$ with $z$ eventually smaller than the sheath thickness. Then, the new created electron can also gain energy in the sheath and may eventually also create electron-ion pairs. Therefore, the number of ionizing electrons should be multiplied by a factor $m$. Finally the discharge potential $V_{d}$ is expressed by $V_{d}=W / m \gamma_{i}$.

Kadlec and Musil [20] pointed out that there exists an optimized magnetic field shape for trapping as much as possible secondary electrons in the neighbourhood of the target. They found, in case of a planar circular magnetron, that the total magnet strength of the outer magnet should be 1.8 times stronger than the inner magnet strength. In case that the outer magnet strength is weaker, the magnetron is called to be unbalanced type I, while the magnetron is called unbalanced type II when the outer magnet strength is stronger [21]. In case of an unbalanced magnetron type II, not all secondary electrons are efficiently captured in the neighbourhood of the target. Hence, also a higher plasma density far away from the cathode may arise, as seen in figure III.3.4 [22,23]. This higher density plasma away from the cathode is often called a plasma plume. Usually this means that the substrate, and hence the growing film interacts with a higher density plasma.

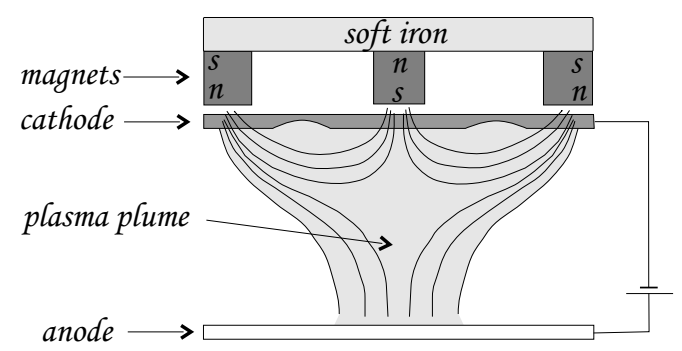

Figure III.3.4: Schematic drawing of an unbalanced type II magnetron. The unbalanced magnet array causes a higher density plasma away from the cathode.

A magnetron discharge has been simulated on a self-consistent way by several groups $[19,24-$ 27].

\section{4: Reactive sputtering}

Sputter deposition of yttria stabilized zirconia (YSZ) and titanium nitride (TiN) films from pure metallic $\mathrm{Zr} / \mathrm{Y}$ or Ti targets is the main subject of this thesis. This means that oxygen or nitrogen gas should be introduced during the deposition in order to get stoichiometric YSZ or 
TiN films respectively. Sputtering with a reactive gas in the plasma is called reactive sputtering.

Although it is counteracted by the sputter erosion itself, the reactive gas will not only react at the substrate and the chamber walls, but also at the target. Essentially no reactions occur in the gas phase, i.e. as the sputtered atoms travel from the target to the substrate, because it is impossible to conserve both energy and momentum in a two-body reaction. This modification of the target by a chemical reaction has its influence on the sputter process since the sputter yield $Y$ and the ISEE coefficient $\gamma_{i}$ are both material dependent (see III.2 and III.3).

Since the reactive gas initially reacts with the metallic adparticles on the chamber walls and the substrate, the gas is "getter pumped" into the film. This means that when increasing the reactive gas flow, the total pressure will not increase significantly until a critical flow is reached. This critical flow is reached when the metallic adparticles can't getter all the reactive gas, and an excess of the reactive gas will remain in the gas phase. When further increasing the reactive gas flow, the total pressure will increase linearly. The slope of that linear increase depends inversely on the pumping speed. At the same time, the excess of reactive gas in the gas phase may react with the target. Since the ISEE coefficient $\gamma_{i}$ depends on the target material, a change in the discharge voltage $V_{d}$ will be observed. The experimentally measured influence of an increasing oxygen flow on the total pressure $P$ and the discharge voltage $V_{d}$ during the sputtering from a $\mathrm{Zr} / \mathrm{Y}$ target is shown in figure III.4.1. In this case, the critical reactive gas flow was 2.5 à $3 \mathrm{sccm}$.

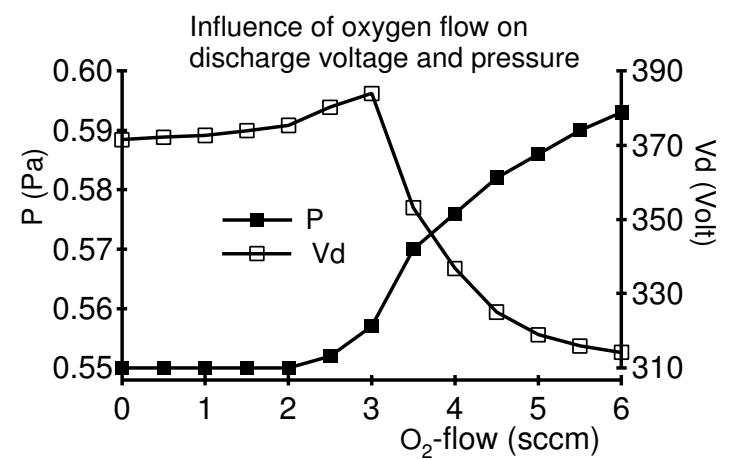

Figure III.4.1: Influence of an increasing $\mathrm{O}_{2}$-flow on the discharge voltage $V_{d}$ and the total pressure $P$ during the sputtering of a $\mathrm{Zr} / \mathrm{Y}(85 / 15 \mathrm{Wt} \%)$ target. The discharge current was kept at $0.4 \mathrm{~A}$.

Many authors have reviewed the benefits and the problems of reactive magnetron sputtering [28-31]. Berg et al. was the first to model the reactive sputtering of a compound from a metallic target, taking into account the modification of the target by the reactive gas [32]. Berg divided the incoming reactive gas flow $q_{o}$ into three flows: the flow to the target $q_{t}$, the flow to the chamber walls and the substrate $q_{c}$ and the flow of reactive gas that is not gettered at any surface in the sputtering chamber $q_{p}$. This flow $q_{p}$ passes out the chamber through the pump. The relation between the reactive gas partial pressure $P_{R}$, the external pumping speed $S$ and the flow $q_{p}$ is given by $q_{p}=P_{R} S$.

The equations describing the kinetics of the consumption of the reactive gas during reactive sputtering can be solved if the values of $q_{t}$ and $q_{c}$ can be found. Neglecting second-order effects, Berg calculated these mass flows by considering the balance of supply and removal of the reactive gas at the chamber walls (+ substrate) and the target. 


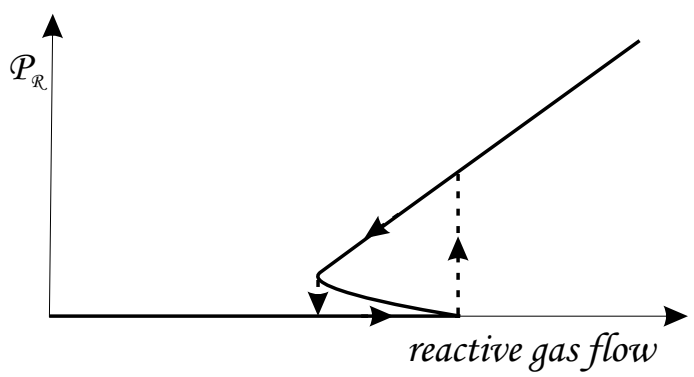

Figure III.4.2: Schematic drawing of a typical influence of the reactive gas flow on the reactive gas partial pressure $P_{R}$ as experimentally observed (dashed line) and as calculated by Berg et al. (full curve).

As already mentioned, the partial pressure $P_{R}$ will initially remain close to zero when increasing the reactive gas flow since all the reactive gas is chemisorbed by the metallic adparticles at the chamber walls and the substrate. As soon as the film on the chamber walls is stoichiometric, an excess of the reactive gas will develop and the partial pressure will increase. Due to this excess of reactive gas, also the target may react. Generally, the sputter yield of a reacted target is lower than the metallic target. Therefore, the reaction of the target with the reactive gas is often called poisoning. The discharge is called to be in metallic mode as long as the reactive gas flow didn't exceed the critical gas flow. At higher gas flow, the discharge is called to be in poisoned mode. This poisoning will decrease the flux of metallic particles towards the walls, decreasing the gettering effect and hence given rise to a larger excess of reactive gas. Therefore, an avalanche effect sets in and the reactive gas partial pressure increases abruptly. As soon as the target is fully reacted, the partial pressure $P_{R}$ will increase linearly with the gas flow. Due to the lower sputter yield of a reacted target, decreasing the reactive gas flow below the critical gas flow will not restore the target in its metallic mode. However, a hysteresis in the reactive gas partial pressure will be observed. This behaviour of the reactive gas partial pressure in function of the reactive gas flow is shown in figure III.4.2. The dashed line is the experimentally observed $P_{R}$, while the full curve represents the $P_{R}$ calculated by Berg. Assuming a lower ISEE coefficient for a metallic than for a reacted target, the discharge voltage should behave as shown in figure III.4.3.

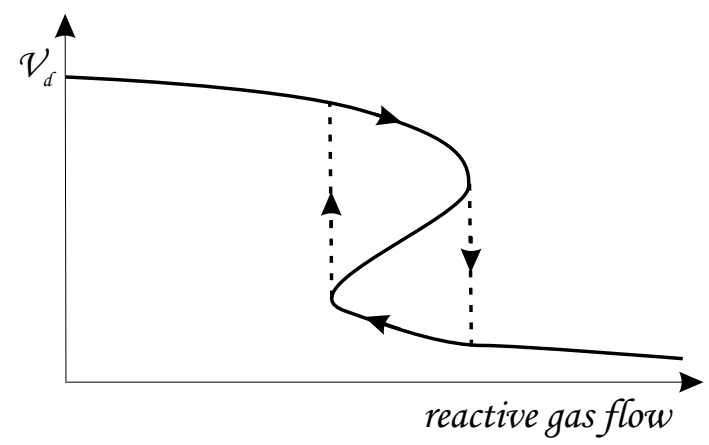

Figure III.4.3: Schematic drawing of a typical influence of the reactive gas flow on the discharge voltage $V_{d}$ as observed experimentally (dashed line) and as calculated by Berg et al. (full curve).

With this simple model, Berg nicely reproduced the influence of several deposition parameters such as ion current density, target area, chamber area, total pressure, pumping speed,... on the critical reactive gas flow [28]. Later on, Li [33] and Pekker [34] adapted the "Berg model" to take into account the influence of the plasma chemistry. Pflug [35] and Matsuda [36], then took into account the influence of a changing ISEE coefficient $\gamma_{i}$ on the ion current density bombarding the target. Finally, Ofner [37] rewrote the Berg model taking into account the energy dependence of the sputter yield $Y$. 
However, there are some drawbacks in the Berg model. Since Berg only takes into account one single effect of the reactive gas on the target, i.e. chemisorption, the Berg model can never explain the observed behavior of the discharge voltage $V_{d}$ in figure III.4.1, i.e. first increasing and then decreasing with increasing $\mathrm{O}_{2}$-flow. Such a $V_{d}$ behavior has been observed by many authors for many materials $[15,38]$. Secondly, the Berg model is also unable to explain the thickness of the reacted layer at the target surface. Berg assumes in his model that the target surface reacts with the reactive gas by chemisorption, hence the reacted layer at the target surface is only one monolayer. However, often a reacted layer of 20 à $30 \AA$ is observed [39]. These two observations indicate that next to chemisorption, another reaction between the reactive gas and the target surface should be taken into account. It has been measured by Depla et al. [40] that the reactive gas will not only chemisorb on the target, but will also get implanted. These implanted reactive gas atoms may react with the target atoms and form a compound, or they may recombine and diffuse outwards or they can stay in the target as non-reacted reactive gas atoms [41]. Again the condition of the target (metallic, compound or chemisorbed by reactive gas) and the substrate (metallic or compound) can be calculated in function of the reactive gas flow [41]. Similar influences of the deposition parameters on the critical gas flow are obtained. Moreover, due to the presence of both chemisorption and implantation, an increase followed by a decrease of the discharge voltage $V_{d}$ with increasing reactive gas flow can be understood. This because it has been measured that chemisorbed reactive gas causes a decrease of the ISEE coefficient $\gamma_{i}$ and hence an increase of the discharge voltage $V_{d}[17,42]$. On the other hand, it is shown that compound formation causes an increase or decrease of the ISEE coefficient $\gamma_{i}$ and hence a decrease or increase of the discharge voltage $V_{d}[15,43]$, depending on the material. Secondly, also the thickness of the reacted target surface of 20 à $30 \AA$ can be understood, since this is in the same range as the depth of the implanted reactive gas atoms.

According to the models of Berg and Depla, only a stochiometric film can be deposited on the substrate if the reactive gas flow is at least the critical reactive gas flow. However, in our deposition system, a local reactive gas inlet is used. This local gas inlet is positioned just in front of the substrate. Therefore, a local higher partial pressure of the reactive gas is created, offering the possibility to deposit stoichiometric films on the substrate, without having a reaction between the target and the reactive gas.

\section{5: Deposition system}

The previous sections described briefly how reactive magnetron sputtering can be used to deposited stoichiometric compounds. As already mentioned, all YSZ and TiN films in this thesis are deposited with this technique. Before describing the depositions and the obtained results (chapter V to VIII), an overview of the used setup is given (see fig. III.5.1).

Since magnetron sputtering is a vacuum technique, the deposition chamber is pumped down to a base pressure of about $5 \times 10^{-6}$ mbar before starting the deposition. A rotary $\left(30 \mathrm{~m}^{3} / \mathrm{h}\right)$ and roots pump $\left(270 \mathrm{~m}^{3} / \mathrm{h}\right)$ are used to pump down to $2 \times 10^{-3}$ mbar, after which a turbo molecular $(1500 \mathrm{l} / \mathrm{s})$ and a diaphragm pump $\left(6.5 \mathrm{~m}^{3} / \mathrm{h}\right)$ are taking over. Between the turbo pump and the chamber, a butterfly valve allows to adjust the pumping speed.

The gas inlet of the Ar and the reactive gas is controlled by mass flow controllers.

The base pressure was measured by a dual (pirani + penning) gauge, while the pressure during the deposition was measured by a capacitance gauge. 


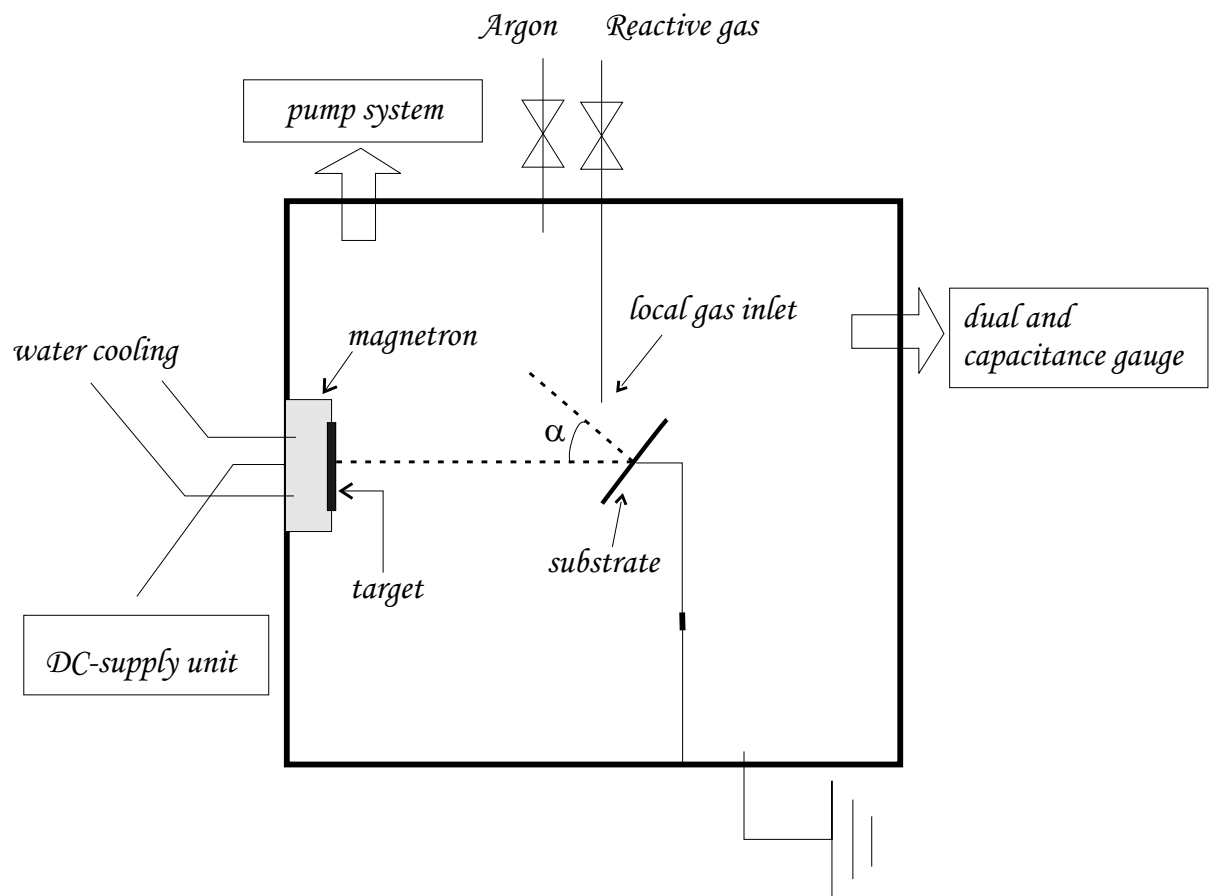

Figure III.5.1: Setup of the used deposition system.

An unbalanced planar, circular home made magnetron was used. The total field of the outer magnet is nine times stronger than the total field of the inner magnet, as such the magnetron is called a 1/9 magnetron. A $5 \mathrm{~cm}$ diameter, 3 or $4 \mathrm{~mm}$ thick metallic target, with a purity of $99.999 \%$ and $99.995 \%$ for $\mathrm{Ti}$ and $\mathrm{Zr} / \mathrm{Y}$ respectively was clamped on the cathode. A stainless steel $\left(5 \times 7 \mathrm{~cm}^{2}\right)$ substrate holder was used. The substrate holder could be biased, heated and also tilted with respect to the material flux. The target-substrate angle $\alpha$ is defined as the angle between the substrate normal and the target normal.

The substrate itself was a polycrystalline stainless steel strip $\left(2 \times 5 \mathrm{~cm}^{2}\right)$ clamped on the substrate holder together with a glass substrate $\left(1 \times 2 \mathrm{~cm}^{2}\right)$. A line was drawn with a marker on the glass substrate, creating a step in the deposited film after cleaning the glass substrate in ethanol. The height of this step, and thus the film thickness, was measured by a stylus method. The film deposited on the stainless steel strip was used for characterization. Only for TEM measurements, the glass substrate was used. Between the target and the substrate, a stainless steel plate $\left(8 \times 20 \mathrm{~cm}^{2}\right)$ acted as shutter during the magnetron run up, and the first minutes of the discharge. Thereafter, the shutter was moved away starting the deposition.

The magnetron was powered by a DC supply unit, especially designed for magnetron sputtering.

\section{References}

1: P. Sigmund: Phys. Rev. 184(2) (1969) p.383-415

2: M.W. Thompson: Vacuum 66 (2002) p.99-114

3: V.S. Smentkowski: Prog. Surf. Sci. 64 (2000) p.1-58

4: J.E. Mahan, A. Vantomme: J. Vac. Sci. Technol. A 15(4) (1997) p.1976-1989

5: H. Urbassek in "Plasma-Surfac interactions and processing of materials" Nato series E vol.176 published by Kluwer Academic Publishers ISBN: 0792305841

6: P. Sigmund in "Sputtering by particle bombardment I" published by Springer-Verlag ISBN: 0387105212

7: Stopping Range of Ions in Matter version 2003.20, www.srim.org 
8: W. Eckstein in "Computer simulation of ion-solid interactions" published by SpringerVerlag ISBN: 0687190570

9: M.T. Robinson, I.A. Torrens: Phys. Rev. B 9(12) (1974) p.5008-5024

10: D.E. Harrison: J. Appl. Phys. 52(6) (1981) p.4251-4258

11: M.T. Robinson in "Sputtering by particle bombardment I" published by Springer-Verlag ISBN: 0387105212

12: H.E. Roosendaal in "Sputtering by particle bombardment I" published by Springer-Verlag ISBN: 0387105212

13: S.M. Rossnagel, H.R. Kaufman: J. Vac. Sci. Technol. A 4(3) (1986) p.1822-1825

14: D.J. Field, S.K. Dew, R.E. Burrell: J. Vac. Sci. technol. A 20(6) (2002) p.2032-2041

15: W. Westwood in "Sputter deposition" published by AVS, ISBN: 0735401055

16: M. Ohring in "Materials science of thin films: deposition and structure" second edition published by Academic Press ISBN: 0125249756

17: B. Chapman in "Glow Discharge Processes" published by John Wiley \& sons ISBN: 0 $47107828 \mathrm{X}$

18: M.A. Lieberman, A.J. Lichtenberg in "Principles of plasma discharge and materials processing" published by John Wiley \& sons ISBN: 0471005770

19: G. Buyle, D. Depla, K. Eufinger, J. Haemers, W. De Boscher, R. De Gryse: J. Vac. Sci. Technol. A 21(4) (2003) p.1218-1224

20: S. Kadlec, J. Musil: J. Vac. Sci. Technol. A 13(2) (1995) p.389-393

21: B. Windows, N. Savvides: J. Vac. Sci. Technol. A 4(2) (1986) p.196-202

22: P.J. Kelly, R.D. Arnell: Surf. Coat. Technol. 108-109 (1998) p.317-322

23: B. Windows, N. Savvides: J. Vac. Sci. Technol. A 4(3) (1986) p.453-456

24: G. Buyle, W. De Boscher, D. Depla, K. Eufinger, J. Haemers, R. De Gryse: Vacuum 70 (2003) p.29-35

25: G. Buyle, D. Depla, K. Eufinger, J. Haemers, W. De Boscher, R. De Gryse: Vacuum 74 (2004) p.353-358

26: I. Kolev, A. Bogaerts: Contrib. Plasma Phys. 44(7-8) (2004) p.582-588

27: S. Kondo, K. Nanbu: J.Phys.D: Appl. Phys. 32 (1999) p.1142-1152

28: S. Berg, T. Nyberg: Thin Solid Films 476 (2005) p.215-230

29: J. Musil, P. Baroch, J. Vlcek, K.H. Nam, J.G. Han: Thin Solid Films 475 (2005) p.208218

30: W.D. Sproul, D.J. Christie, D.C. Carter : Thin Solid Films 491 (2005) p.1-17

31: I. Safi : Surf. Coat. Technol. 127 (2000) p.203-219

32: S. Berg, H-O. Blom, T. Larsson, C. Nender: J. Vac. Sci. Technol. A 5(2) (1987) p.202207

33: C. Li, J.H. Hsieh, W.M. Huang: Surf. Coat. Technol. 198 (2005) p.372-378

34: L. Pekker: Thin Solid Films 312 (1998) p.341-347

35: A. Pflug, B. Szyszka, V. Sittinger, J. Niemann: Proceedings of 46th annual SVC technical conference, SVC 2003, p.241-247

36: Y. Matsuda, K. Otomo, H. Fujiyama: Thin Solid Films 390 (1-2) (2001) p.59-63

37: H.Ofner, R. Zarwasch, E. Rille, H.K. Pulker: J. Vac. Sci. Technol. A 9 (1991) p.27952796

38: T. Hata, H. Matsuda, R. Ando, S. Horita: Jpn. J. Appl. Phys. 33-2 (3B) (1998) L455-L458

39: D. Depla, J. Haemers, R. De Gryse: "Discharge voltage behaviour during reactive sputtering of oxides" accepted for publication in Thin Solid Films.

40: D. Guttler, B. Abendroth, R. Grotzschel, W. Moller, D. Depla: Appl. Phys. Lett. 85 (25)

(2004) p.6134-6136

41: D. Depla, R. De Gryse: Surf. Coat. Technol. 183 (2004) p.184-203

42: D. Depla, R. De Gryse: Plamsa Sources Sci. Technol. 10 (2001) p.547-555 
43: D. Depla, S. Heirwegh, S. Mahieu, J. Haemers, R. De Gryse: "Understanding the discharge voltage behaviour during reactive sputtering of oxides" submitted. 


\section{Chapter IV: Biaxially aligned films and their characterization methods.}

\section{1: Introduction}

In the preceding chapters, the thin film growth in general (chapter I) and the relation between the elementary processes and fundamental phenomena of structure formation and the resulting microstructure (chapter II) has been described. The scope of this thesis is to investigate the mechanism of biaxial alignment in thin films deposited by unbalanced magnetron sputtering. The deposition technique itself has been described in more detail in chapter III. Before discussing the obtained results of the deposited films and proposing a growth mechanism (chapters V, VI and VII), it will be discussed in more detail what is meant by biaxial alignment and how these biaxially aligned thin films were characterized. Scanning electron microscopy (SEM) has been used to form a picture of the global topography or microstructure. Transmission electron microscopy (TEM) was used to investigate the local structure more in detail and also to correlate the local crystallographic orientation with the microstructure. X-ray Diffraction (XRD) and Transmission Electron Diffraction are both used to measure the biaxial alignment and are briefly discussed in the following sections. XRD provides us data about the average crystallographic orientation of the thin films, while transmission electron diffraction enables us to investigate the local crystallographic orientation. Since I have not the intention nor the knowledge to fully describe both techniques, the interested reader is referred to e.g. [1-6] for more details.

\section{2: Biaxial alignment}

Deposition on an amorphous or random oriented polycrystalline substrate results in a polycrystalline thin film, at least if the mobility during the deposition is high enough to ensure a completed nucleation into crystalline islands (chapter I and II). Polycrystalline means that the film is a collection of crystalline grains or columns. Each crystalline grain is characterized by its crystallographic orientation with respect to the substrate. The direction perpendicular to the substrate is called the out-of-plane orientation. Perpendicular to this out-of-plane orientation, an in-plane orientation can be defined.

A film is called to be [hkl] out-of-plane aligned or to have a preferential [hkl] out-of-plane orientation if a substantial fraction of the grains has a [hkl] out-of-plane orientation, in which $\mathrm{h}, \mathrm{k}$, and $\mathrm{l}$ represent the Miller indices. The degree of preferential out-of-plane orientation is determined by $\mathrm{N}_{\text {hkl }} / \mathrm{N}_{\text {tot }}$, in which $\mathrm{N}_{\text {tot }}$ is the volume of all grains and $\mathrm{N}_{\mathrm{hkl}}$ the volume of the grains with the $[\mathrm{hkl}]$ direction perpendicular to the substrate. A schematic representation of such an out-of-plane alignment is shown in figure IV.2.1.a.
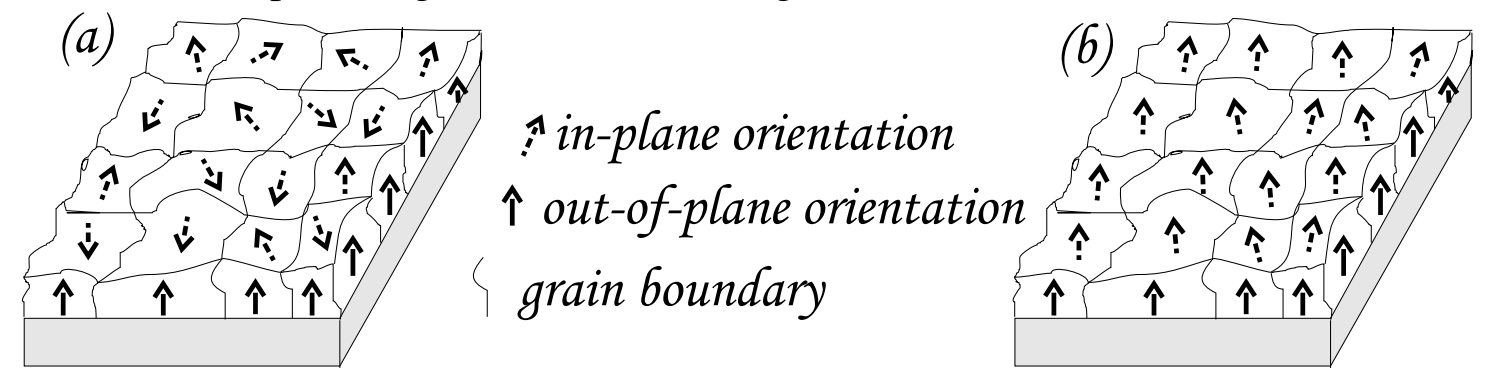

Figure IV.2.1: Schematic drawing of an out-of-plane aligned (a) and a biaxially aligned (b) thin film. The full arrows indicate the crystallographic out-of-plane orientation, while the dashed arrows indicate the inplane orientation. The angular spread on the in-plane orientations determines the degree of in-plane alignment. 
The definition of a preferential out-of-plane orientation, i.e. if a "substantial" fraction of the grains has a specific out-of-plane orientation, is rather broad. In practice, a film is called outof-plane aligned if the degree of out-of-plane alignment $\mathrm{N}_{\mathrm{hk}} / \mathrm{N}_{\text {tot }}$ is at least 0.75 . Otherwise, also the other observed out-of-plane orientations are mentioned.

It is possible that the grains not only have a crystallographic out-of-plane alignment, but also a certain alignment in a direction parallel to the substrate. It means that all grains have nearly the same crystallographic orientation pointing parallel to a reference direction in the substrate plane. Such a film is called biaxially aligned. In case of a cubic material, fixing two different directions implies the complete determination of the crystallographic orientation of the grain. A schematic drawing of such a biaxially aligned film is shown in figure IV.2.1.b. The angular spread on the in-plane orientations determines the degree of in-plane alignment.

\section{3: X-ray Diffraction}

$\mathrm{X}$-rays are electromagnetic radiation with a wavelength in the range of 0.5-2.5 $\AA$ [3]. In our case, $\mathrm{Cu} \mathrm{K \alpha} \mathrm{X}$-rays with a wavelength of $1.54 \AA$ are used.

An electron in an alternating electromagnetic field will oscillate with the same frequency as the field. Therefore, when an X-ray beam hits an atom, the electrons around the atom start to oscillate with the same frequency as the incoming beam. In almost all directions destructive interference will occur, this means that the combining waves are out of phase and there is no resultant energy leaving the solid sample. However, the atoms in a crystal are arranged in a regular pattern, and in a very few directions constructive interference will occur. The waves will be in phase and there will be well defined X-ray beams leaving the sample at various directions. Hence, a diffracted beam may be described as a beam composed of a large number of scattered X-rays mutually reinforcing one another.

However, this model is rather complex to handle mathematically and is often simplified to the interpretation of X-ray Bragg reflections from a series of parallel planes inside the crystalline solid. Consider an X-ray beam incident on a pair of parallel (hkl) planes $P_{1}$ and $P_{2}$, separated by an interplanar distance $d_{h k l}$ as shown in figure IV.3.1. Two parallel incident X-rays $A$ and $B$ make an angle $\theta$ with these planes. A reflected beam of maximum intensity will result if the waves represented by $A^{\prime}$ and $B^{\prime}$ are in phase. This means that the difference in path length between $A$ to $A^{\prime}$ and $B$ to $B^{\prime}$ should be an integral number $\mathrm{n}$ of wavelengths $\lambda$. This is expressed by Bragg's law: $2 d_{h k l} \sin \theta=\mathrm{n} \lambda$.

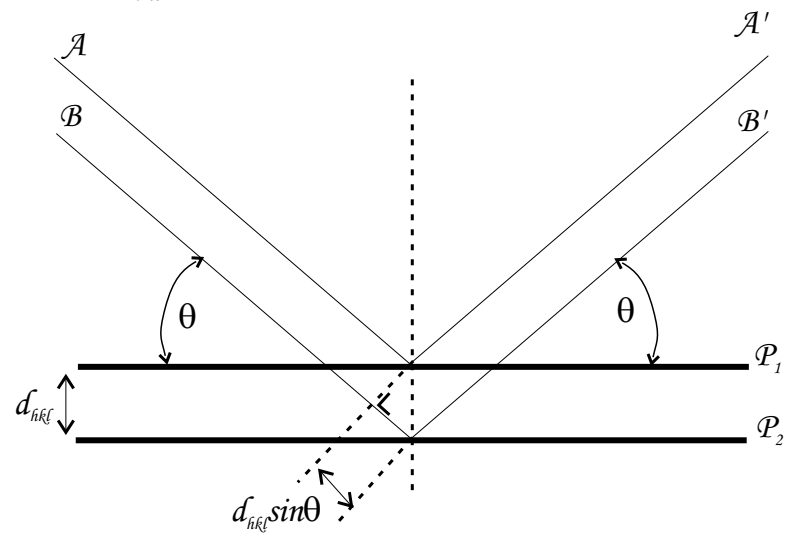

Figure IV.3.1: Derivation of Bragg's law.

In a typical diffractometer, the angle between the incoming X-rays and the substrate plane on the one hand and the angle between the substrate plane and the X-ray detector on the other hand is always equal. A typical set-up of a $\theta / \theta$ goniometer is shown in figure IV.3.2. 
This equality of the source and detector angle $\theta$ implies that only crystallographic planes parallel to the substrate plane can be observed. Hence, by increasing the angle $\theta$, a spectrum of all crystallographic planes parallel to the substrate plane can be measured. Such a measurement is usually called a $\theta / 2 \theta$ spectrum.

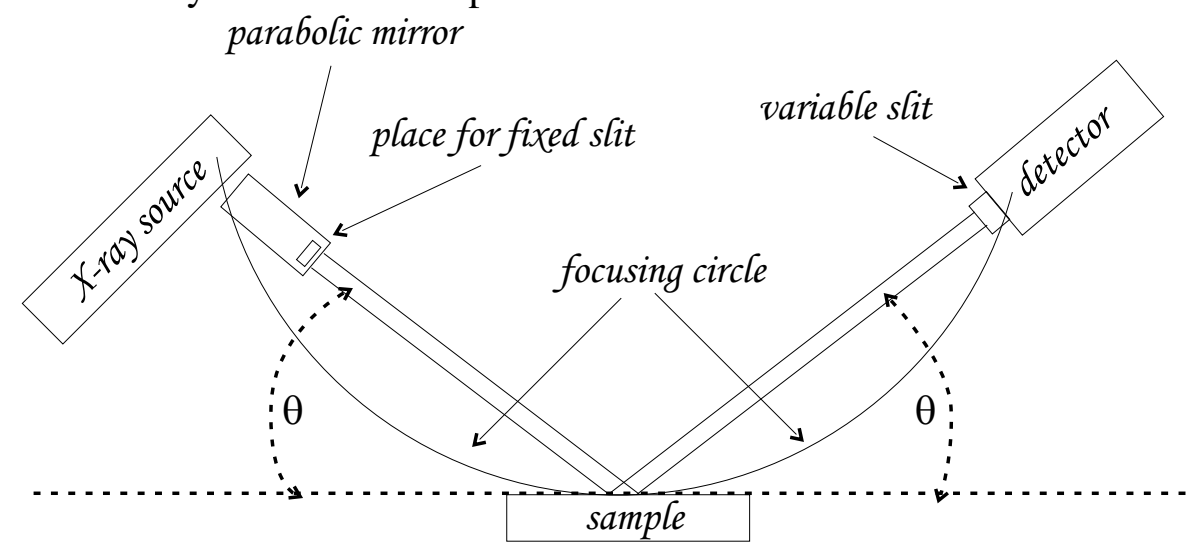

Figure IV.3.2: Scheme of the used set-up of a $\theta / \theta$ diffractometer.

Knowing the angle $\theta$ (or $2 \theta$ ) at which the diffraction peak is observed, reveals to which crystallographic set of planes $\{\mathrm{hkl}\}$ this peak belongs since each set of planes $\{\mathrm{hkl}\}$ is characterized by its interplanar distance $d_{h k l}$. The diffraction position $\theta$ and the interplanar distance $d_{h k l}$ are related by Bragg's law. A typical $\theta / 2 \theta$ spectrum of TiN film is shown in figure IV.3.3.

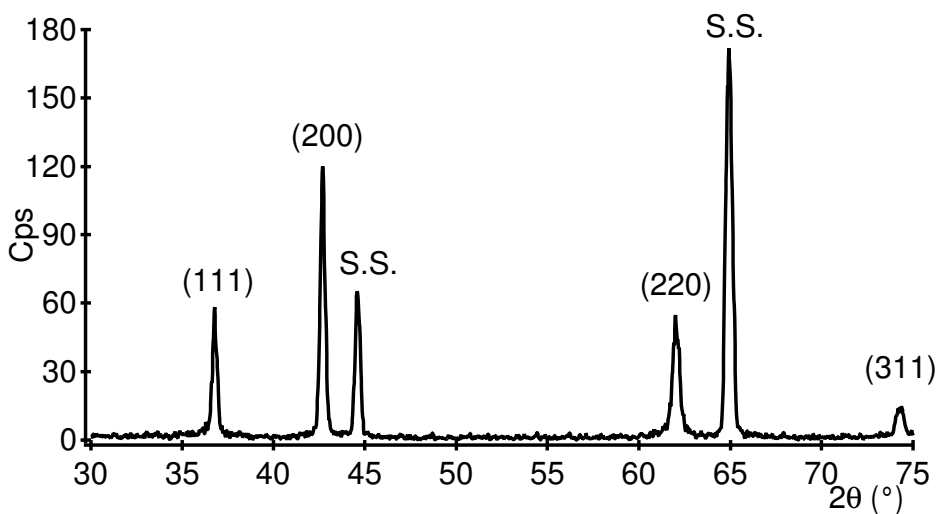

Figure IV.3.3: Typical $\theta / 2 \theta$ spectrum of TiN. Several diffraction peaks at different $2 \theta$ positions are observed. The corresponding crystallographic plane for each diffraction peak is indicated in the brackets. Also the stainless steel (S.S.) substrate peaks are indicated.

Since a $\theta / 2 \theta$ spectrum reveals which crystallographic planes are parallel to the substrate plane, this set-up is ideal for measuring the out-of-plane orientation of the polycrystalline film. However, the intensity (or area) of a diffraction peak (hkl) is not directly related to the number of grains with a (hkl) plane parallel to the substrate plane because the intensity is influenced by several other parameters [1-3]. Therefore, the intensities of the diffraction peaks have to be recalculated before knowing the degree of out-of-plane alignment.

In theory, the correlation between the intensity of a diffraction peak (hkl) and the number of grains with a (hkl) plane parallel to the substrate can be calculated taking into account the multiplicity factor, the structure factor, the absorption factor, the temperature factor and the Lorentz-polarisation factor [1-3]. All these factors together give the intensity factor $I_{h k l}$ of a (hkl) plane, which indicates the relative "efficiency" for diffracting an X-ray beam. Fortunately, these intensity factors don't have to be calculated but are tabulated by the International Centre for Diffraction Data (ICDD). Unfortunately, there is one drawback since these intensity factors not only depend on the examined material, but also on the used 
experimental set-up. As indicated in figure IV.3.2, several slits can be placed after the X-ray source and before the detector. In our set-up a variable slit at detector side is used. Such a variable slit ensures that always X-rays originating from the same area of the substrate are examined, independent of the angle $\theta$. At the source side, no slit or a fixed slit is used. The use of no slit or a fixed slit implies that the radiated area of the substrate decrease when increasing the source angle $\theta$.

The tabulated intensity factors of the ICDD database are valid for a diffractometer only using fixed slits on both sides. The tabulated intensity factors for variable slits $I_{v a r}$ can be recalculated from the intensity factors for fixed slits $I_{f i x}$ by the relation $I_{v a r}=I_{f i x} \sin \theta$ in case that a variable slit is used on the detector as well as on the source side [7]. Since in our geometry only the detector side is equipped with a variable slit, this relation should be adjusted.

This adjusted relation between $I_{f i x}$ and $I_{v a r}$ has been experimentally measured by taking a $\theta / 2 \theta$ spectrum of corundum and $\mathrm{Fe}_{2} \mathrm{O}_{3}$ powder. A powder is assumed to be random out-of-plane oriented and hence to have peak intensities directly related to the intensity factors. In this way, the relation $I_{v a r}=I_{f i x}(\sin \theta)^{0.42}$ was obtained. The ratio of the measured peak intensities in function of $\sin \theta$ for both powders is shown in figure IV.3.4. In the next chapters, the calculated degree of out-of-plane alignment uses these re-calculated peak intensities. Finally, the degree of the (hkl) out-of-plane alignment is calculated by $A_{h k l} I_{h k l} / \sum A_{h k l} I_{h k l}$ with $A_{h k l}$ the peak area of the (hkl) peak in the $\theta / 2 \theta$ spectrum, $I_{h k l}$ the intensity factor for the used system (one variable slit) and $\Sigma$ the summation over all observed diffraction peaks of the film material.

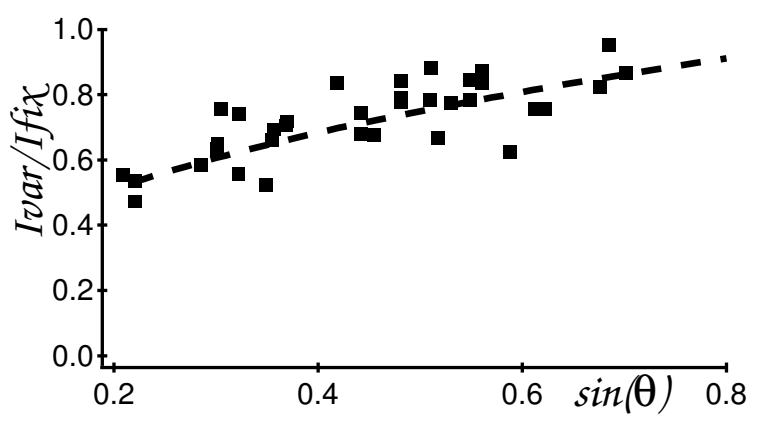

Figure IV.3.4: Experimental results for the ratio $I_{v a r} / I_{f i x}$ of a corundum and $\mathrm{Fe}_{2} \mathrm{O}_{3}$ powder in function of $\sin \theta$ (black markers). The fitted correlation is indicated by the dashed line.

Not only the out-of-plane alignment can be measured by a diffractometer, but also the inplane alignment. This in-plane alignment is measured by means of a pole figure. Such a pole figure is scanned by measuring the diffraction intensity of a given (hkl) reflection (with source and detector at constant $\theta$ ) at a large number of different angular orientations of the sample. A contour map of the intensity is then plotted as a function of angular orientation of the specimen. The intensity of a given (hkl) reflection is proportional to the number of (hkl) planes in reflecting condition (Bragg's law). Hence, the pole figure gives the probability of finding a given crystallographic orientation as a function of the specimen orientation. The most common representation for such a pole figure is a stereographic projection [8]. The systematic change in angular direction of the sample is normally achieved by utilizing a four-circle goniometer. The intensity data for various settings of Phi and Chi are collected. Usually, all Phi values for a given Chi are measured, after which Chi is changed and the process is repeated. The angles Phi and Chi are explained in figure IV.3.5. 


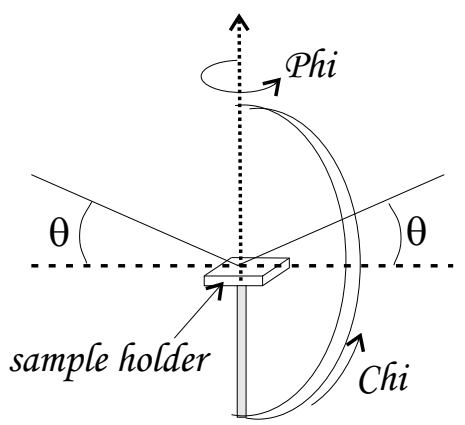

Figure IV.3.5: Representation of the angles $\theta$, Phi and Chi in a four-circle goniometer.

In case of a totally random oriented film the intensity in the measured pole figure should be uniform. However, in case of a [hkl] out-of-plane aligned film, a (hkl) pole figure should at least give an intense peak around $\mathrm{Chi}=0$. This intense peak is called a pole. Figure IV.3.6.a shows an example of a (111) pole figure of a [111] out-of-plane aligned TiN film. In case that this film has a random in-plane alignment, a (200) pole figure gives an intense ring at Chi $=$ $55^{\circ}$ (see fig. IV.3.6.b), since the angle between the [111] direction and a [200] direction is $55^{\circ}$ (see fig. IV.3.6.c).

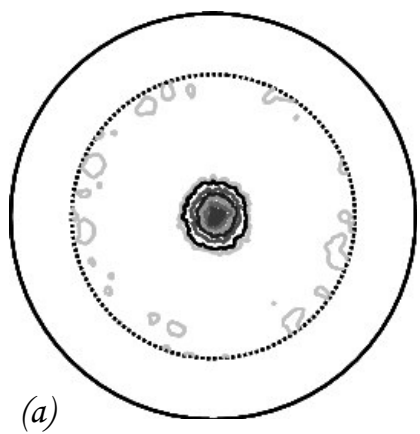

Figure IV.3.6: A (111) pole figure (a) a alignment, but with random in-plane orientation. Also the angle between a [111] and a [200] direction in case of a cubic material is presented (c).

As soon as an in-plane alignment arises, this diffraction ring dissolves into several diffraction spots or poles. An example of a (200) pole figure of a biaxially aligned [111] out-of-plane aligned TiN film is shown in figure IV.3.7.a. In chapter I ( $\$$ I.3, section surface energy) it was discussed that TiN grains are terminated by $\{100\}$ planes if they grow according to their kinetically limited growth shape, and if the $\{100\}$ planes have the lowest growth rate. Hence, figure IV .3.7.b shows the corresponding geometric orientation of a $\{100\}$ faceted TiN grain. The correlation between the pole figure and the TiN grain is clearly observed.
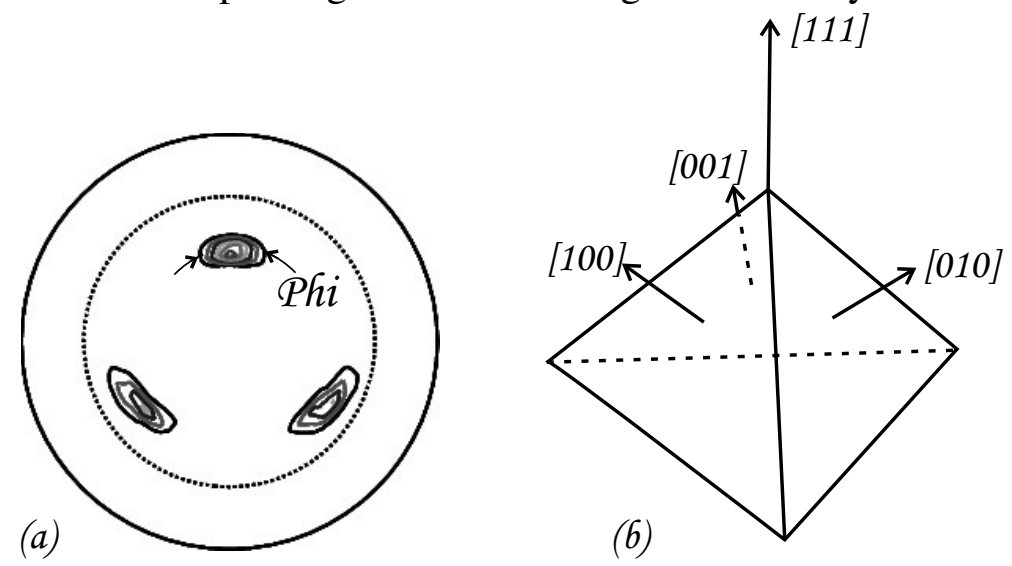

Figure IV.3.7: A (200) pole figure of a biaxially aligned [111] out-of-plane aligned TiN film (a). Also the corresponding orientation of a $\{100\}$ terminated TiN grain is shown (b). 
The degree of in-plane alignment is determined by the full width at half maximum (FWHM) in the Phi direction of a pole, as indicated on figure IV.3.7.a. In practice, during the measurement of a pole figure, not all Phi and Chi positions can be measured, but only in discrete steps. Our pole figures were measured in steps of $5^{\circ}$ or $2^{\circ}$, resulting in a experimentally checked error of $\sim 5 \%$ in the FWHM.

\section{4: Transmission Electron Microscopy and Diffraction}

Similar to X-rays, an electron beam can be treated as an electromagnetic wave. Hence, radiating a solid with electrons will also cause the electrons in the solid to oscillate with the same frequency as the incident electrons. In case of a crystalline solid, this causes again constructive interference and the exit of well defined diffracted electron rays. Similar to the $\mathrm{X}$-rays, constructive diffraction will occur if the incident electron beam satisfies the Bragg law: $\mathrm{n} \lambda=2 d \sin \theta$. However, in case of accelerated electrons, their wave length $\lambda$ depends on their energy [4]. In case of $400 \mathrm{keV}$ electrons, the wavelength is $1.644 \mathrm{pm}$. Since this wavelength is about two orders smaller than the $\mathrm{Cu} \mathrm{K \alpha}$ wavelength, the angle at which the constructive diffraction occurs will be very small $(\lambda \sim \sin \theta \sim \theta)$.

In case of a Transmission Electron Microscope (TEM), a beam of energetic electrons is bombarded on a thin specimen. After transmission through the specimen, a series of electromagnetic lenses focuses them on a fluorescent screen. A great advantage of such a TEM is that as well as an enlarged image as a diffraction pattern can be recorded. A schematic diagram of a TEM in imaging and in diffraction mode is shown in figure IV.4.1.

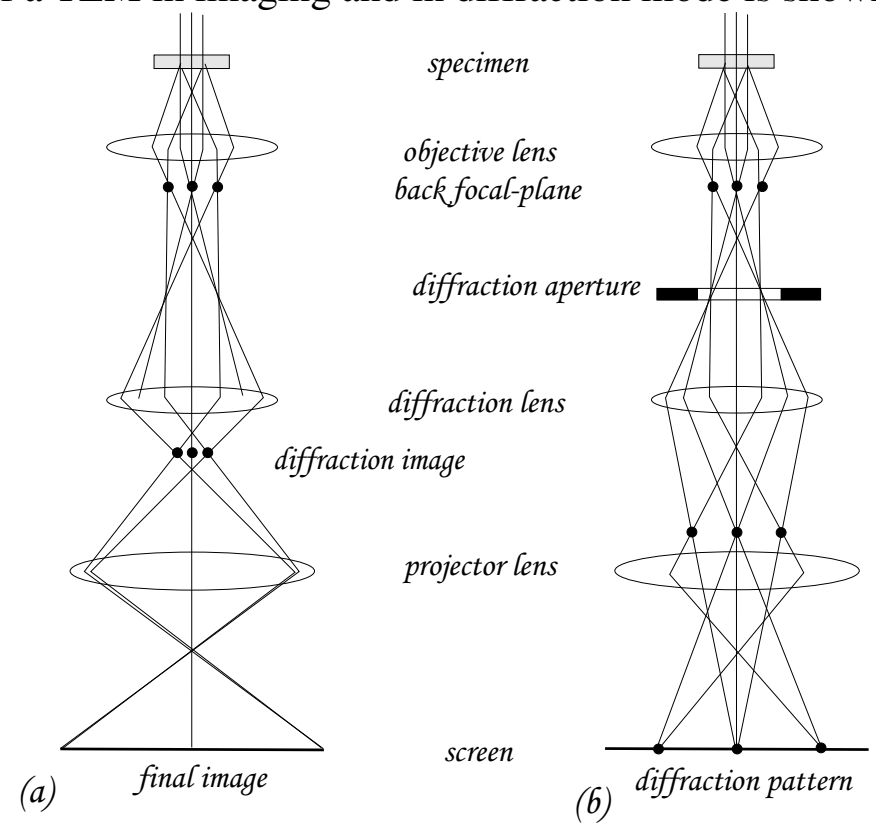

Figure IV.4.1: Schematic diagram of a TEM system in imaging (a) and diffraction (b) mode.

The specimen is placed in a holder in the front focal-plane of the objective lens. The magnified image produced by the objective in its image plane serves as an object for the diffraction lens, which produces a second intermediate image, which is further magnified by the projector lens to produce the final image on the fluorescent screen. In the diffraction mode, the strength of the diffraction lens is reduced so that the image of the diffraction pattern in the back focal-plane of the objective lens is focused on the final screen. In other words, the 'object' for the diffraction lens in the diffraction mode is the diffraction pattern produced by the objective lens. 
By selecting the diameter of the diffraction aperture $\mathrm{D}$, a diffraction pattern of a selected area with diameter $\mathrm{D} / \mathrm{M}$ of the specimen can be obtained. $\mathrm{M}$ is the magnification of the objective lens. This technique is called selected area diffraction (SAED).

Instead of varying the source and detector angle $\theta$, as in X-ray diffraction, the source of electrons is stationary and the detector is a fluorescent screen. In case of a random oriented polycrystalline film, the diffraction pattern will consist of diffraction rings. However, in case of an aligned film, the rings will dissolve into diffraction spots. Hence, electron diffraction gives an idea of the alignment of the film. A typical example of a diffraction pattern of a TiN film is shown in figure IV.4.2.
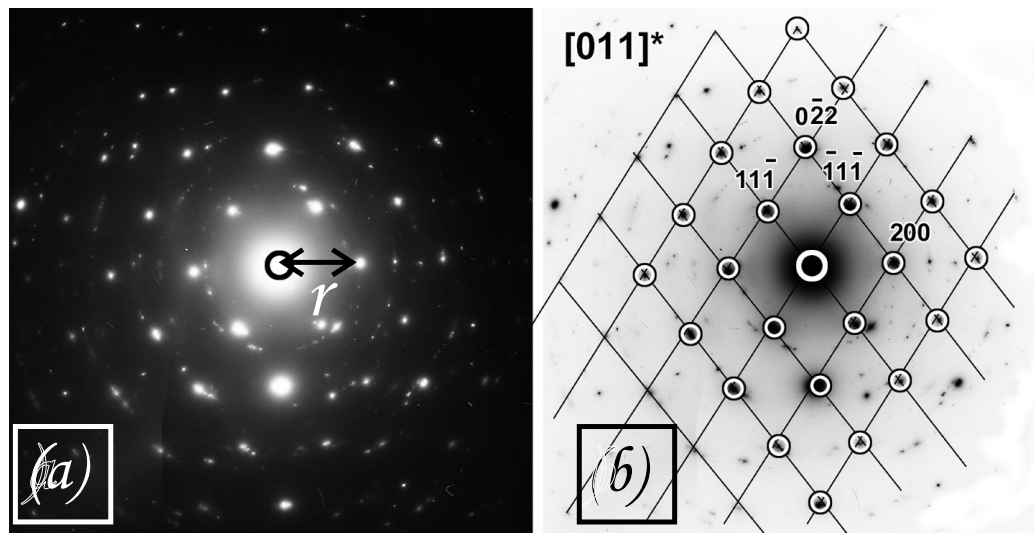

Figure IV.4.2: Example of a diffraction pattern taken with the SAED technique (a). The diffraction pattern was taken from a cross-section view of a biaxially aligned TiN film, with the e-beam incident along the [011] direction. On the right side, a scheme of the indexing is given.

In order to know which spot belongs to which crystallographic plane, a correlation should be found between the radial distance to the centre $r$ and the interplanar distance $d_{h k l}$. Therefore, the camera length $L$, which is a characteristic for the optics of the microscope is needed (see fig. IV.4.3). It should be mentioned that the camera length $L$ is not a physical distance, but a 'projected' distance which varies with the settings of the electromagnetic lenses.

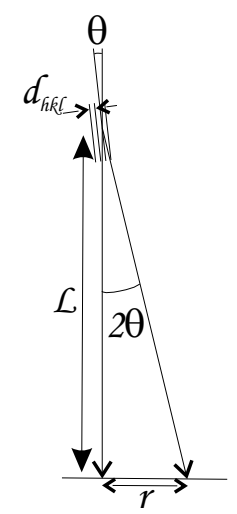

Figure IV.4.3: Geometry for the electron diffraction and definition of the camera length $L$.

According to Bragg's law: $2 d_{h k l} \sin \theta=\mathrm{n} \lambda$. Since the diffraction angle $\theta$ is very small for low order diffractions of $400 \mathrm{keV}$ electrons: $\sin \theta \sim \tan \theta \sim 1 / 2 \tan (2 \theta)$. By the geometry in figure IV.4.3: $\tan (2 \theta)=r / L$. Hence Bragg's law simplifies to $r d_{h k l}=\lambda L$, which allows to determine an interplanar distance $d_{h k l}$ by measuring the separation of diffraction spots $r$, at least if the camera length $L$ is known.

Finally, after determining to which crystallographic planes the spots belong, it can also be determined along which crystallographic direction(s) or zone axis ('s) the electron beam was 
penetrating the specimen. This because the electron diffraction of a crystalline solid oriented with a specific crystallographic direction towards the e-beam will produce a characteristic standard diffraction pattern. Some of these standard diffraction patterns for a fcc material are shown in figure IV.4.4. Hence, electron diffraction allows also to reveal the crystallographic out-of-plane orientation and the in-plane orientation. The shape of the spots gives an idea of the degree of alignment.
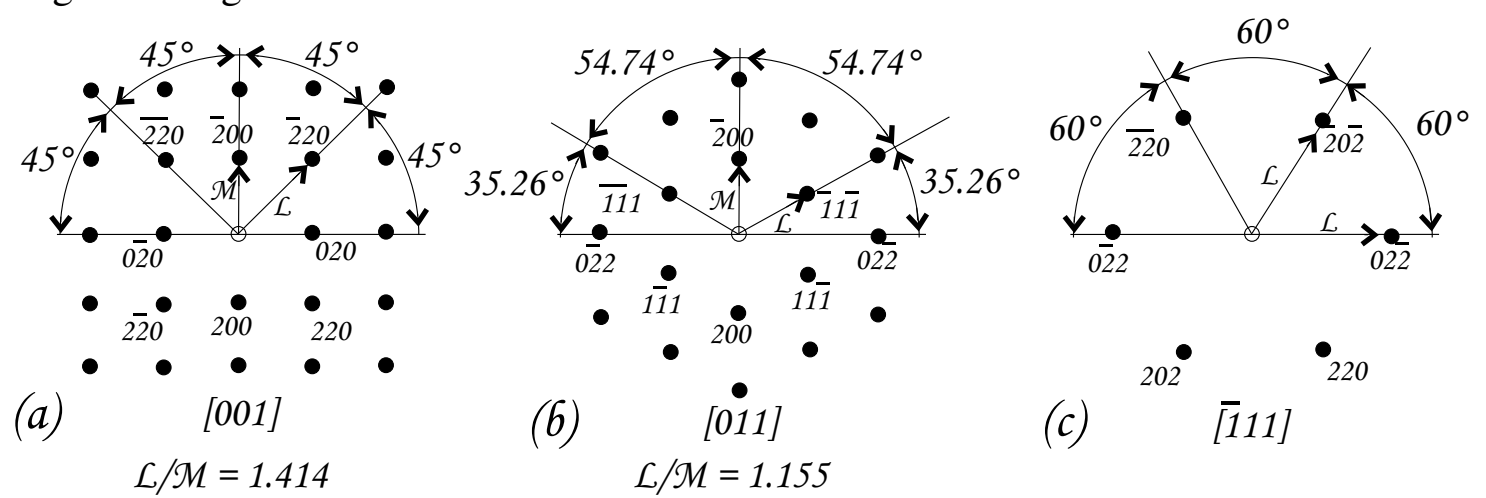

Figure IV.4.4: Three standard diffraction patterns for an fcc material with the incident e-beam oriented along a [001] (a), [011] (b) and a [111] (c) orientation. L and M are indicated on the figures.

A TEM makes use of electromagnetic lenses. Such electromagnetic lenses produce a rotation between the object (specimen) and the image. As the rotation depends upon the lens excitation, the image rotates on the viewing screen as the magnification is changed. Since one of the purposes of the TEM is to correlate the image with the crystallographic information from the diffraction pattern, the rotation between the image and the diffraction pattern should be known.

\section{References}

1: R. Jenkins, R.L. Snyder in "Introduction to X-ray powder diffractometry" Chem. Analysis Vol.138, published by John Wiley \& sons, ISBN: 0-471-51339-3

2: A.J.C. Wilson in "Elements of X-ray crystallography" published by Addison-Wesley

3: B.D. Cullity in "Elements of X-ray Diffraction" published by Addison-Wesley, ISBN: 0201-01174-3

4: P.E. Champness in "Electron Diffraction in the transmission electron microscope" published by Bios Scientific, ISBN: 1859961479

5: B. Fultz, J.M. Howe in "Transmission electron microscopy and diffractometry of materials" published by Springer, ISBN: 3-540-43764-9

6: D.B. Williams, C.B. Carter in "Transmission Electron Microscopy, a textbook for materials science" part I-IV, published by Plenum press, New York, ISBN 0306453242

7: Users Manual Brüker-AXS D8: PDF Maint V.9.0 powder diffraction database manager 2003.

8: O. Johari, G. Thomas in "Stereographic projection and its applications" Vol. IIa in techniques of Metal research, published by Interscience Publishers, SBN 471122001 


\section{Chapter V: Mechanism of out-of-plane alignment.}

\section{1: Introduction}

The scope of this thesis is understanding the biaxial alignment of thin films deposited by unbalanced magnetron sputtering. As discussed in chapter IV, an important part of this biaxial alignment is the out-of-plane alignment.

Therefore, the out-of-plane orientation in sputter deposited Yttria Stabilized Zirconia (YSZ) and Titanium Nitride (TiN) will be discussed in this chapter. In literature, the out-of-plane orientation of sputter deposited TiN thin films has been widely investigated, at least in comparison to the out-of-plane orientation of YSZ. Although the numerous reports about the influence of several deposition parameters on the out-of-plane orientation of TiN, not that much models explaining the real mechanism were developed. First, the experimental observations for TiN (section V.2) and YSZ (section V.3) will be discussed and summarized (section V.4). In the next sections, the experimental results of our deposited films will be compared with the published growth models. It will be shown that some of these models cannot explain the obtained results (V.5.1 and V.5.2), while other models only partially (V.5.3). Hence, a new, more elaborated model unravelling the mechanism of out-of-plane alignment had to be developed (section V.6). Comparing the obtained results of TiN with the results of YSZ enables to develop a more general model. This because TiN has a rocksalt structure [1], while YSZ is fluorite like [2].

Since the resulting out-of-plane orientation of a deposited film strongly depends on the fundamental structure forming phenomena, the new developed model will often refer back to the developed SZM (table II.5).

\section{2: Experimental observations: TiN}

TiN thin films were deposited with the system described in chapter III. As such, a $5 \mathrm{~cm}$ diameter Ti target (99.999\% purity) was sputtered in an $\mathrm{Ar} / \mathrm{N}_{2}$ atmosphere. The discharge was ignited in pure Ar with closed shutter, after which the $\mathrm{N}_{2}$-flow was increased. The $\mathrm{N}_{2}$ inlet was local and in the neighbourhood of the substrate. After a few minutes, the shutter was opened and the deposition started. The total flow was kept constant at $60 \mathrm{sccm}$ and the pressure at $0.55 \mathrm{~Pa}$. The discharge current was 0.9 A. The substrate (a stainless steel (S.S.) strip and glass) was kept at grounded potential and at floating temperature. The deposition time was adapted in order to obtain films of about $1 \mu \mathrm{m}$. The target-substrate distance was 13 $\mathrm{cm}$ and the substrate was not tilted with respect to the target $\left(\alpha=0^{\circ}\right)$. A series of depositions at identical conditions, but with varying $\mathrm{Ar} / \mathrm{N}_{2}$ flow ratio was carried out.

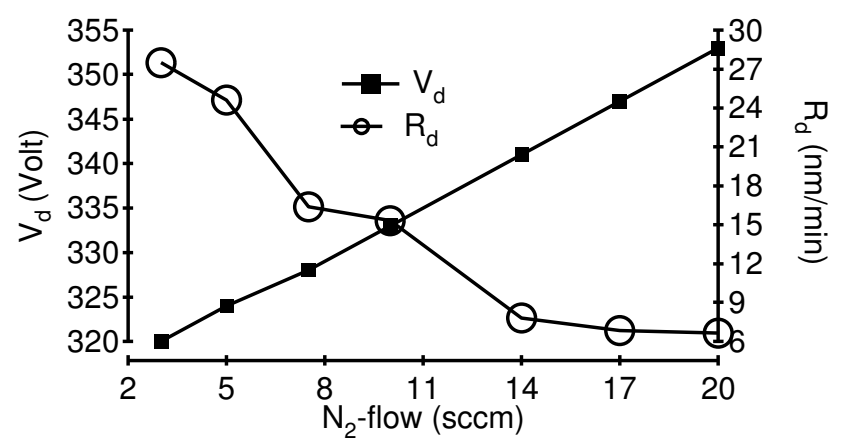

Figure V.2.1: Influence of the $\mathrm{N}_{2}$-flow on the discharge voltage $\left(\mathrm{V}_{\mathrm{d}}\right)$ and the deposition rate $\left(\mathrm{R}_{\mathrm{d}}\right)$ for TiN depositions at $0.55 \mathrm{~Pa}, \mathrm{~T}-\mathrm{S}$ distance $13 \mathrm{~cm}, \alpha=0^{\circ}$ and $\mathrm{I}_{\mathrm{d}}=0.9 \mathrm{~A}$. 
Increasing the $\mathrm{N}_{2}$-flow from 3 to $20 \mathrm{sccm}$ resulted in an increasing discharge potential (in absolute value) and a decreasing deposition rate (see figure V.2.1). An $\mathrm{N}_{2}$-flow lower than 3 sccm resulted in $\mathrm{Ti}$ or substoichiometric $\mathrm{TiN}_{\mathrm{x}}$ as observed by XRD $\theta / 2 \theta$ spectra. The substrate temperature is floating, meaning that the substrate is not cooled nor heated. However, due to the energy flux towards the substrate, the temperature measured by a backside clamped thermocouple raised up to $250-300^{\circ} \mathrm{C}$, i.e. a homologous temperature $\mathrm{T} / \mathrm{T}_{\mathrm{m}}$ of 0.15 in case of TiN.

Table V.2.2 lists the global deposition conditions of the TiN depositions.

Table V.2.2:Global deposition conditions of TiN.

\begin{tabular}{|l|l|}
\hline Total gas pressure & $0.55 \mathrm{~Pa}$ \\
\hline Ar-flow & $57-40 \mathrm{sccm}$ \\
\hline $\mathrm{N}_{2}$-flow & $3-20 \mathrm{sccm}$ \\
\hline Total gas flow & $60 \mathrm{sccm}$ \\
\hline Deposition temperature & Floating \\
\hline Deposition rate & $27.5-6.7 \mathrm{~nm} / \mathrm{min}$ \\
\hline Deposition time & $35-130 \mathrm{~min}$ \\
\hline Film thickness & $1+/-0.1 \mu \mathrm{m}$ \\
\hline Target-substrate angle $\alpha$ & $0^{\circ}$ \\
\hline Discharge voltage $\mathrm{V}_{\mathrm{d}}$ & $320-353 \mathrm{~V}$ \\
\hline Discharge current $\mathrm{I}_{\mathrm{d}}$ & $0.9 \mathrm{~A}$ \\
\hline Substrate bias & $0 \mathrm{~V}$ \\
\hline
\end{tabular}

All deposited films resulted in a polycrystalline structure, giving rise to clear diffraction peaks in an XRD $\theta / 2 \theta$ spectrum. These XRD spectra of the TiN films on stainless steel are shown in figure V.2.3. For clarity, they were shifted in the vertical direction with increasing $\mathrm{N}_{2}$-flow. The crystallographic TiN directions are indicated at the corresponding diffraction peaks, as also the peaks originating from the substrate.

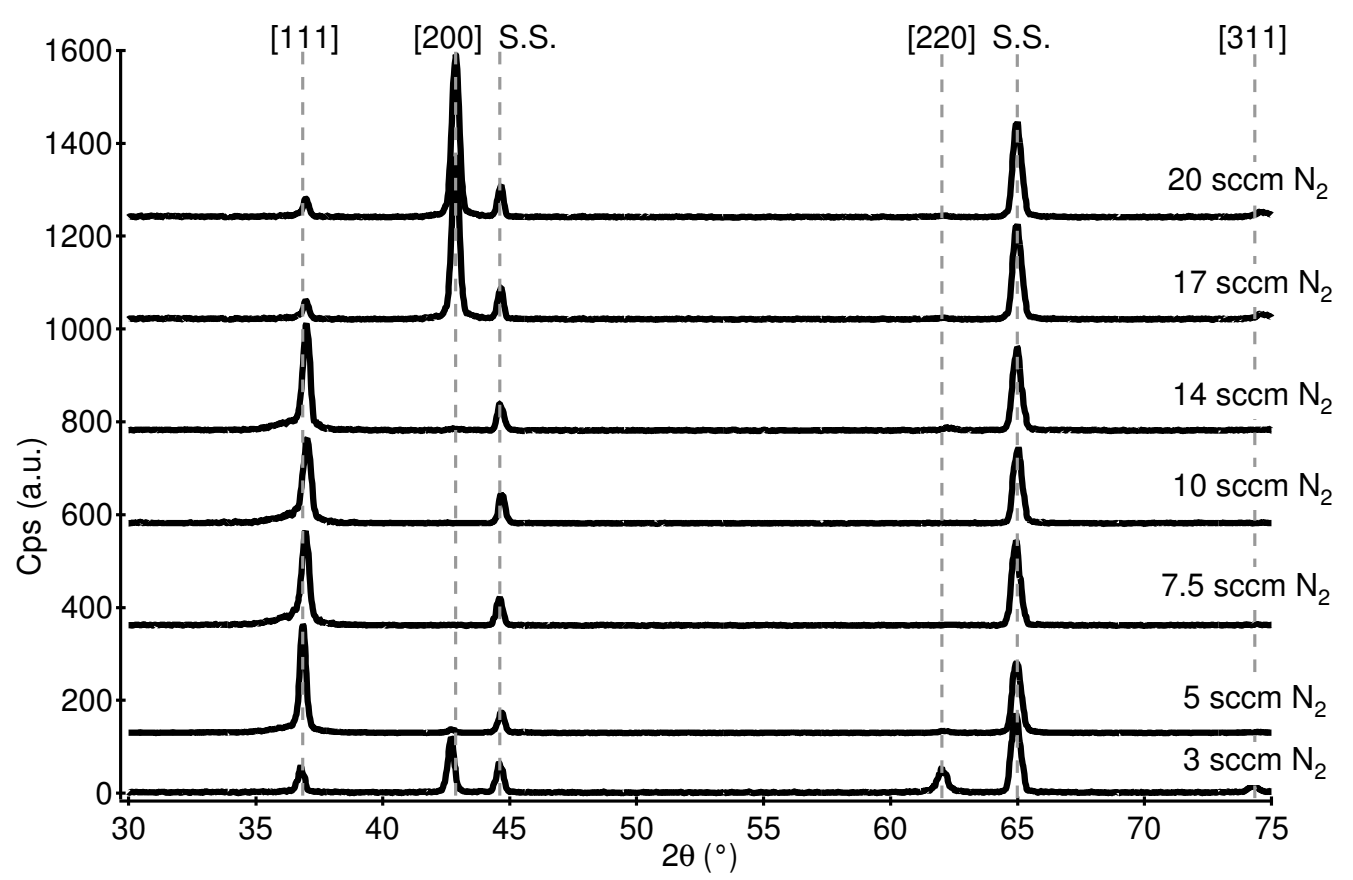

Figure V.2.3: $\theta / 2 \theta$ spectra of TiN layers of $\sim 1 \mu \mathrm{m}$ deposited on stainless steel at different $\mathrm{N}_{2}$-flow. For clarity, the spectra are shifted in the vertical direction. A clear transition from random oriented to a [111] and finally [200] out-of-plane alignment with increasing $\mathrm{N}_{2}$-flow is observed.

As discussed in chapter IV, the normalized fraction of grains with a specific crystallographic out-of-plane orientation can be calculated from these $\theta / 2 \theta$ spectra, taking into account the 


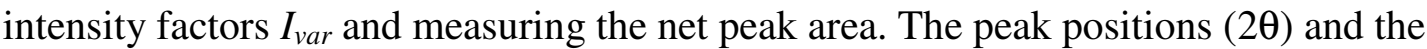
calculated intensity factors $I_{v a r}$ for the different TiN crystallographic planes, according to the ICDD data card $\mathrm{n}^{\circ}$ : 00-038-1420 [3] are shown in table V.2.4.

Table V.2.4: Peak positions and intensity factors $I_{v a r}$ for different TiN crystallographic planes.

\begin{tabular}{|c|c|c|}
\hline plane & $I_{\text {var }}$ & $2 \theta$ \\
\hline$[111]$ & 67.77 & 36.663 \\
\hline$[200]$ & 100 & 42.597 \\
\hline$[220]$ & 52.05 & 61.814 \\
\hline$[311]$ & 23.50 & 74.07 \\
\hline
\end{tabular}

The normalized fraction of grains with a specific out-of-plane orientation as a function of the $\mathrm{N}_{2}$-flow is shown in figure V.2.5. It is clearly seen that the deposition at $3 \mathrm{sccm} \mathrm{N}_{2}$ exhibit a nearly random out-of-plane orientation. However increasing the $\mathrm{N}_{2}$-flow resulted in nearly perfect [111] out-of-plane aligned TiN films. Between 14 and $17 \mathrm{sccm} \mathrm{N}_{2}$, a sudden change from the [111] out-of-plane alignment to the [200] out-of-plane alignment occurs.

Hence, it can be concluded that the deposited TiN films at increasing $\mathrm{N}_{2}$-flow can be divided into three regimes: random oriented $(3 \mathrm{sccm})$, [111] out-of-plane oriented $(5-14 \mathrm{sccm})$ and [200] out-of-plane oriented ( $>17 \mathrm{sccm}$ ). A film of each regime has been characterized by scanning electron microscopy (SEM) and transmission electron microscopy (TEM).

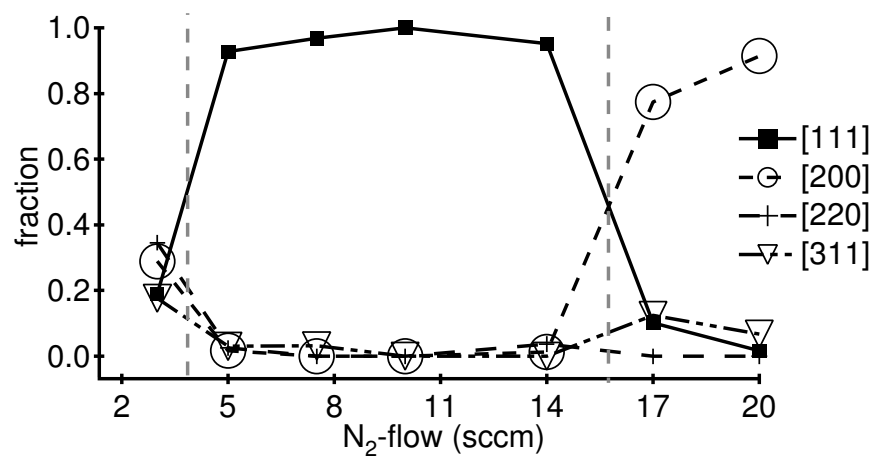

Figure V.2.5: Normalized fraction of grains with a specific out of-plane orientation as a function of the $\mathrm{N}_{2}$-flow, calculated from the $\theta / 2 \theta$ spectra in figure V.2.2.

Figure V.2.6 shows a plan view SEM image of a TiN film deposited at $3 \mathrm{sccm} \mathrm{N_{2 }}$. It is clearly observed that the film consists of well faceted grains. It is also observed that re-nucleation occurs time by time since small crystallites are noticed on the facets of grains, as indicated by the white circles on the SEM picture (V.2.6).

As discussed in chapter II, re-nucleation occurs only due to a low adparticle mobility or due to the presence of impurities. Since this re-nucleation is not observed for the depositions at higher $\mathrm{N}_{2}$-flow, it can be concluded that they are not due to an impurity effect but due to a low mobility. As mentioned in chapter I, faceting of the grains will only occur if the mobility of the adparticles is high enough to aim for their most stable position at a grain. Since both processes are statistical processes, it can be concluded that the deposition is carried out at the transition region between faceting and re-nucleation. Generally, the terminating planes of faceted grains will be the planes of lowest growth rate. Hence, if faceting occurs, all the grains will be faceted by the same crystallographic planes and will all have the same crystal habit. Since it is observed in figure V.2.6 that the grains grow randomly with a top, an edge or a plane upwards, the SEM picture is in accordance with the random out-of-plane orientation as measured by XRD. This random out-of-plane orientation, the faceted structure and the sometimes occurring re-nucleation indicates that the deposited film can be catalogued as a deposition of the low mobility side of zone Ic (see table II.5). 


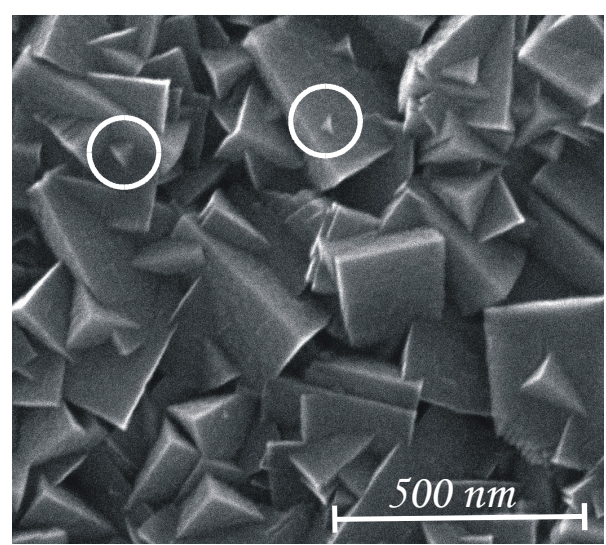

Figure V.2.6: Plan view SEM image of a TiN film deposited on stainless steel at $3 \mathrm{sccm} \mathrm{N}_{2}$. A clear faceted structure with occasionally occurring re-nucleation (white circles) is observed. This microstructure is determined to be zone Ic.

Figure V.2.7 shows a plan view SEM image of the TiN film deposited at $5 \mathrm{sccm} \mathrm{N}_{2}$. Again, a clear faceted structure is observed. However, no re-nucleation is seen. It is also observed that all grains point with a top upwards. Taking into account a $\{200\}$ faceted crystal habit, this observation corresponds with the [111] out-of-plane alignment as observed by XRD.

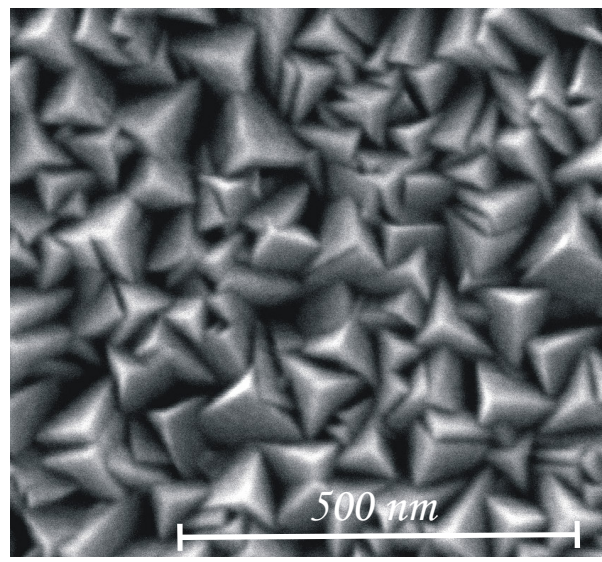

Figure V.2.7: Plan view SEM image of a TiN film deposited on stainless steel at $5 \mathrm{sccm} \mathrm{N}_{2}$. A clear faceted structure is observed, in which all grains point with a top upwards in accordance with the [111] out-of-plane alignment.

Figure V.2.8 shows TEM images of a cross-section of TiN deposited on glass at $5 \mathrm{sccm} \mathrm{N}_{2}$, together with the corresponding diffraction patterns. In figure V.2.8a, the first $300 \mathrm{~nm}$ of the TiN film is shown. It is observed that the grains evolve into columns and that the size of the columns increases with increasing film thickness. In chapter II, this microstructure was described as evolutionary overgrowth. Figure V.2.8b shows the upper few hundred nm of the same TiN film. It is observed that the size of the columns is still increased and that these column tops are well faceted, in accordance with the SEM image. Diffraction patterns of these areas indicate that the film nucleates with random out-of-plane oriented grains. This is seen in figure V.2.8c, because of the diffraction rings. The diffraction pattern of the upper part of the film only indicates [111] out-of-plane oriented grains, in accordance with the XRD observations. Hence, it can be concluded that the [111] out-of-plane alignment is a consequence of an evolutionary overgrowth. In chapter II, it was described that a film characterized by $\mathrm{V}$-shaped columns, terminated by a faceted column top and exhibiting a clear out-of-plane alignment originating from an overgrowth mechanism is deposited in zone T conditions (see table II.5). 


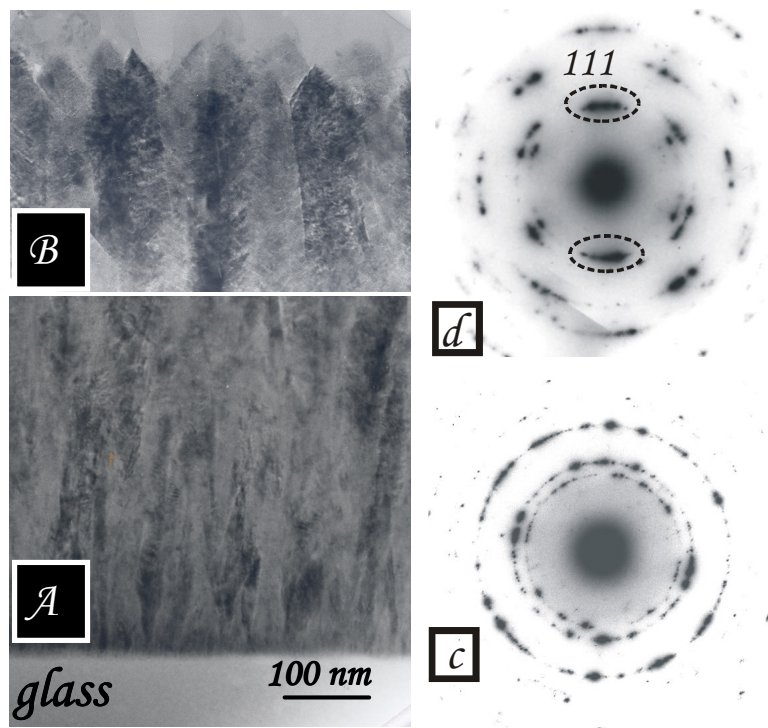

Figure V.2.8: TEM results of a cross-section of a TiN film deposited on glass with an $\mathrm{N}_{2}$-flow of 5 sccm. Part a shows the microstructure of the lower part, while part b shows the microstructure of the upper part of the film. A clear columnar and faceted structure is observed. Part $\mathrm{c}$ and $\mathrm{d}$ are SAED patterns of the lower and upper part of the TiN film. Part c shows a random orientation, while part d shows a clear [111] out-of-plane orientation.

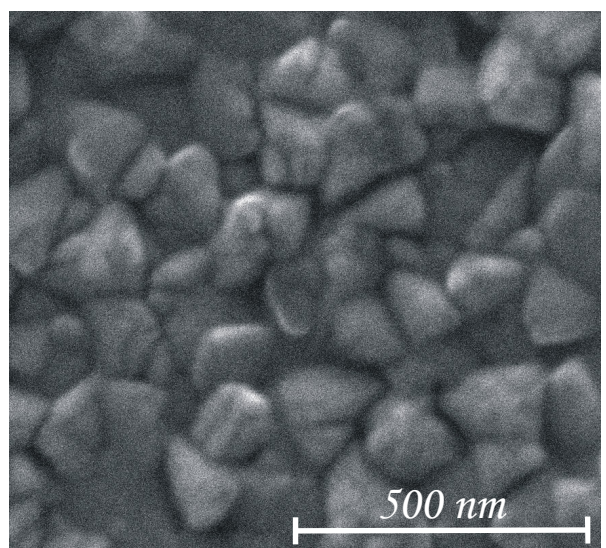

Figure V.2.9: Plan view SEM image of a TiN film deposited on stainless steel at $20 \mathrm{sccm} \mathrm{N}_{2}$. No faceting at the surface is observed.

Figure V.2.9 shows a SEM plan view image of a TiN film deposited at $20 \mathrm{sccm} \mathrm{N}_{2}$. The grains show no longer a faceted structure, but seems to have a blurred or rather rounded surface. TEM results of a cross-section of a TiN film deposited on glass with an $\mathrm{N}_{2}$-flow of 20 $\mathrm{sccm}$ are shown in figure V.2.10. Again part $\mathrm{a}$ and $\mathrm{b}$ show the microstructure of the lower and upper few hundred nm of the TiN film respectively. No evolutionary overgrowth is observed, but it seems that the columns penetrate through the whole film thickness. In accordance with the SEM image, the column tops seems to be rounded. Corresponding SAED diffraction patterns of the lower and upper part of the TiN film are shown in figure V.2.10 $\mathrm{c}$ and $\mathrm{d}$ respectively. In both diffraction patterns, a clear [200] out-of-plane alignment is observed, again in accordance with the XRD measurements. It was discussed in chapter II, that a microstructure characterized by columns penetrating through the whole film thickness, with a curved column top and exhibiting a clear preferential out-of-plane orientation are deposited in zone II conditions.

It can be concluded that at the given deposition conditions, an increasing $\mathrm{N}_{2}$-flow results in: - a transition from random out-of-plane orientation, to a clear [111] out-of-plane alignment and finally into a [200] out-of-plane alignment.

- a corresponding transition from zone Ic, to zone T and finally to zone II structure. 


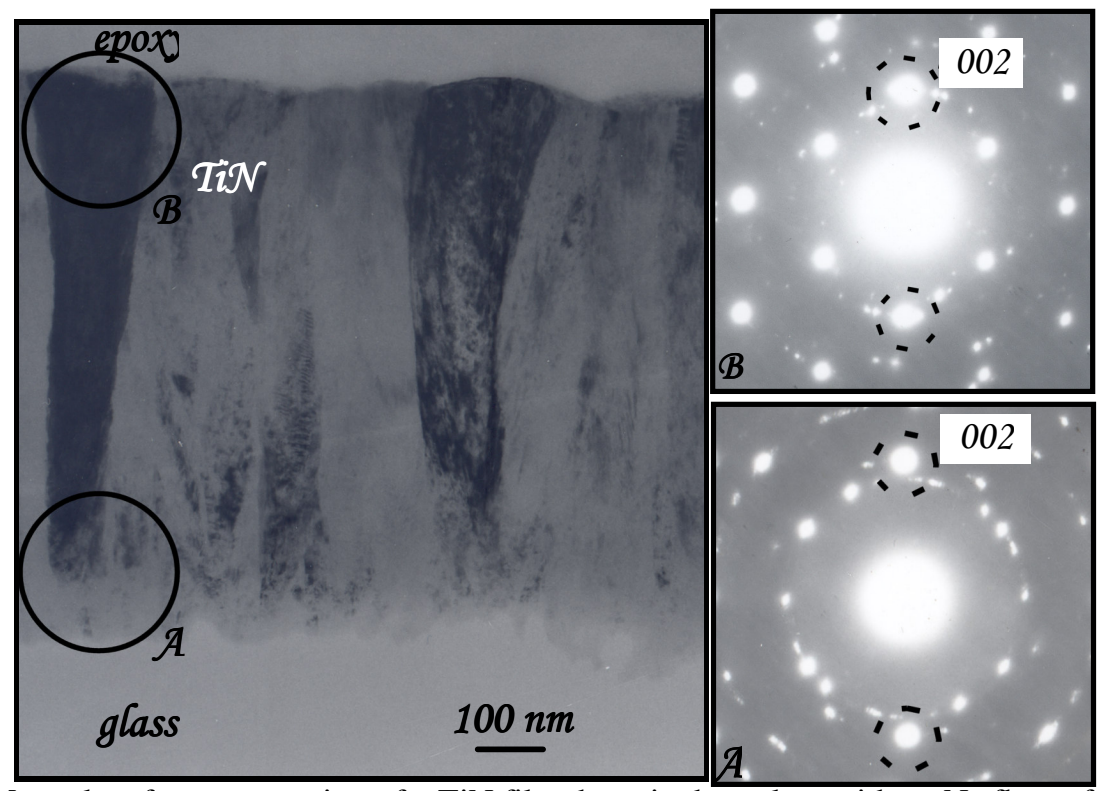

Figure V.2.10: TEM results of a cross-section of a TiN film deposited on glass with an $\mathrm{N}_{2}$-flow of $20 \mathrm{sccm}$. The left part shows the microstructure of the film. A clear columnar structure is observed. The right part are SAED patterns of the lower and upper part of the TiN film. Both SAED patterns show a clear [200] out-of-plane orientation.

As already mentioned, a lot of research to the influence of several deposition parameters on the out-of-plane orientation of TiN films has been published in literature. The transition from a [111] to a [200] out-of-plane alignment with increasing $\mathrm{N}_{2}$-flow is often reported [4-9]. However, also a transition from [111] to [200] out-of-plane alignment with decreasing film thickness [10-13], increasing substrate temperature $[9,14,15]$ or increasing ion-to-atom ratio $[16,17]$ has been observed. Also the influence of the ion energy on the out-of-plane orientation has been investigated [10,18-21].

\section{3: Experimental observations: YSZ}

$\mathrm{ZrO}_{2}$ is monoclinic at room temperature but has a stable fluorite structure above $2370^{\circ} \mathrm{C}$. Doping it with enough $Y$ stabilizes the fluorite structure at room temperature and $\left(\mathrm{ZrO}_{2}\right)_{1-\mathrm{x}}\left(\mathrm{Y}_{2} \mathrm{O}_{3}\right)_{\mathrm{x}}$ or Yttria Stabilized Zirconia is obtained [2]. As observed from the compound formula, doping the $\mathrm{ZrO}_{2}$ fluorite structure with $\mathrm{Y}$ causes the appearance of oxygen vacancies. In our depositions, YSZ films were deposited by reactive sputtering of a $\mathrm{Zr} / \mathrm{Y}$ target $\left(55 / 45 \mathrm{Wt} \%\right.$ ) in an $\mathrm{Ar} / \mathrm{O}_{2}$ gas. Checking the composition of the deposited films by energy dispersive $\mathrm{X}$-ray analysis (EDX) revealed that the ratio of $\mathrm{Zr} / \mathrm{Y}$ in the deposited YSZ films has the same $\mathrm{Zr} / \mathrm{Y}$ ratio as the target. Consequently, a $\left(\mathrm{ZrO}_{2}\right)_{0.715}\left(\mathrm{Y}_{2} \mathrm{O}_{3}\right)_{0.285}$ film with a fluorite like structure in which $11 \%$ of the oxygen is replaced by vacancies is obtained. Nevertheless, for modelling the growth it will be assumed that the deposited YSZ films have a perfect fluorite structure.

Influence of an increasing reactive gas flow:

Similar to the above described TiN depositions, also the influence of an increasing reactive gas flow during the deposition of YSZ was investigated. Therefore, YSZ films were deposited on mirror like polished stainless steel and glass substrates. The discharge current was kept at $0.9 \mathrm{~A}$, the total pressure at $0.55 \mathrm{pa}$, the target-substrate distance at $13 \mathrm{~cm}$ and the targetsubstrate angle $\alpha$ at $0^{\circ}$. The substrate was at floating temperature and at grounded potential. Hence, similar conditions as for the TiN depositions described in section V.2. were used. The $\mathrm{O}_{2}$-flow has been varied between 2 and $7.5 \mathrm{sccm}$, to check if the reactive gas flow again 
changes the microstructure and the out-of-plane alignment. Lowering the $\mathrm{O}_{2}$-flow beneath 2 sccm resulted in bad oxidized YSZ films. Again, the substrate was not cooled nor heated. The energy flux during the substrate resulted again in a heating up of the substrate, up to a homologous temperature $\mathrm{T} / \mathrm{T}_{\mathrm{m}}$ of 0.18 .

The discharge voltage $V_{d}$ and the deposition rate $R_{d}$ changed with increasing reactive gas flow (see fig. V.3.1), indicating the poisoning of the target above $3 \mathrm{sccm} \mathrm{O}_{2}$. The deposition time was adapted in order to obtain films of about $1 \mu \mathrm{m}$. Only the films deposited at 6 and 7.5 sccm had a smaller thickness of only $0.4 \mu \mathrm{m}$ since the very low deposition rate $(<2 \mathrm{~nm} / \mathrm{min})$ required too large deposition times.

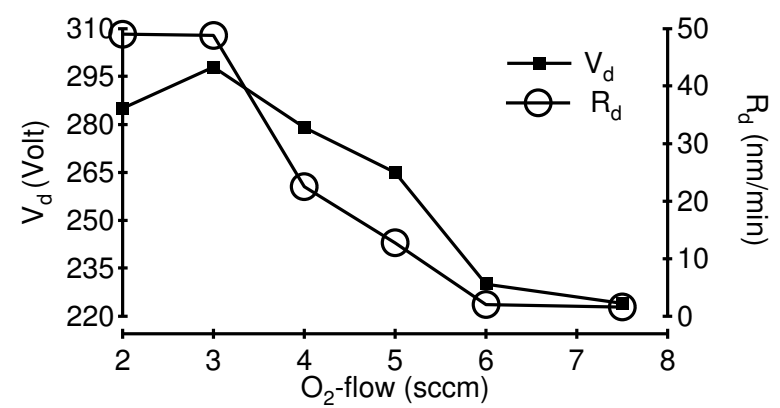

Figure V.3.1: Influence of the $\mathrm{O}_{2}$-flow on the discharge voltage $\left(V_{d}\right)$ and the deposition rate $\left(R_{d}\right)$ for YSZ films deposited at $0.55 \mathrm{~Pa}, \mathrm{~T}-\mathrm{S}$ distance of $13 \mathrm{~cm}, \alpha=0^{\circ}$ and $\mathrm{I}_{\mathrm{d}}=0.9 \mathrm{~A}$.

A list of the global deposition conditions for the YSZ depositions is given in table V.3.2.

Table V.3.2: Global deposition conditions of YSZ.

\begin{tabular}{|l|l|}
\hline Total gas pressure & $0.55 \mathrm{~Pa}$ \\
\hline Ar-flow & $58-52.5 \mathrm{sccm}$ \\
\hline $\mathrm{O}_{2}$-flow & $2-7.5 \mathrm{sccm}$ \\
\hline Total gas flow & $60 \mathrm{sccm}$ \\
\hline Deposition temperature & Floating \\
\hline Deposition rate & $49-2.2 \mathrm{~nm} / \mathrm{min}$ \\
\hline Deposition time & $20-180 \mathrm{~min}$ \\
\hline Film thickness & $1+/-0.1 \mu \mathrm{m}$ \\
\hline Target-substrate angle $\alpha$ & $0^{\circ}$ \\
\hline Discharge voltage $\mathrm{V}_{\mathrm{d}}$ & $295-224 \mathrm{~V}$ \\
\hline Discharge current $\mathrm{I}_{\mathrm{d}}$ & $0.9 \mathrm{~A}$ \\
\hline Substrate bias & $0 \mathrm{~V}$ \\
\hline
\end{tabular}

All deposited films resulted in a polycrystalline structure, giving rise to clear diffraction peaks in the XRD $\theta / 2 \theta$ spectra. These XRD spectra of the YSZ films deposited on a stainless steel substrate are shown in figure V.3.3 for an oxygen flow between 2 and $5 \mathrm{sccm}$. Increasing the $\mathrm{O}_{2}$-flow above $5 \mathrm{sccm}$ did not result in further changes of the XRD $\theta / 2 \theta$ spectra. For clarity, the XRD spectra are shifted in the vertical direction with increasing $\mathrm{O}_{2}$-flow. The crystallographic directions are indicated at the corresponding diffraction peaks. Again the normalized fraction of grains with a specific out-of-plane orientation can be calculated from the $\theta / 2 \theta$ spectra, measuring the area of a diffraction peak and taking into account the intensity factor $I_{v a r}$. The peak positions and the calculated intensity factors $I_{v a r}$ are taken from the ICDD data card $\mathrm{n}^{\circ}$ : 00-030-1468 and are shown in table V.3.4. The mentioned ICCD data card is for a $\left(\mathrm{ZrO}_{2}\right)_{0.85}\left(\mathrm{Y}_{2} \mathrm{O}_{3}\right)_{0.15}$ structure, since no better fitting data card is available. It has been reported by Holzschuh et al. [22] that the unit cell parameter of YSZ increases with increasing $\mathrm{Y}$ content. This is confirmed when comparing the tabled $2 \theta$ values and the $2 \theta$ peak positions in figure V.3.2, since an increasing unit cell parameter corresponds with lower $2 \theta$ values. Although the peak positions shift to lower values, it is assumed that the 
reported intensity factors are still valid for a $\left(\mathrm{ZrO}_{2}\right)_{0.715}\left(\mathrm{Y}_{2} \mathrm{O}_{3}\right)_{0.285}$ structure. The calculated normalized fraction of grains with a specific out-of-plane orientation is shown in figure V.3.5.

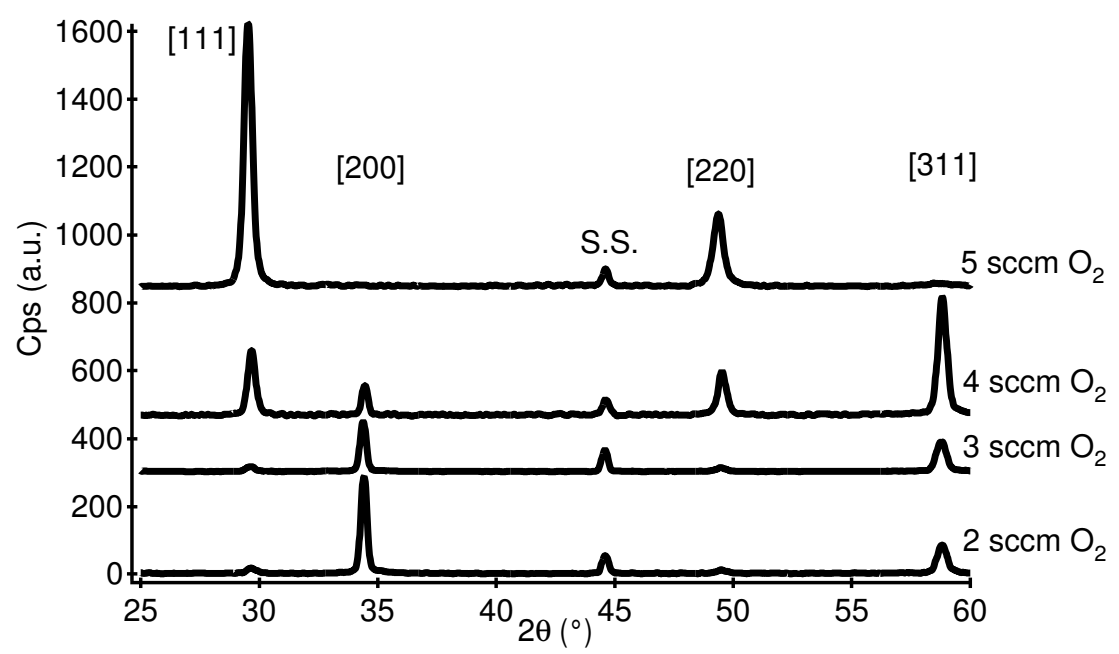

Figure V.3.3: $\theta / 2 \theta$ spectra of YSZ films of $\sim 1 \mu \mathrm{m}$ deposited on stainless steel at different $\mathrm{O}_{2}$-flow. For clarity, the spectra are shifted in the vertical direction. A transition from a [200] out-of-plane alignment to a mixture of

[111] and [220] out-of-plane oriented films with increasing $\mathrm{O}_{2}$-flow is observed.

Table V.3.4: Peak positions and intensity factors $I_{v a r}$ for different $\left(\mathrm{ZrO}_{2}\right)_{0.85}\left(\mathrm{Y}_{2} \mathrm{O}_{3}\right)_{0.15}$ crystallographic planes.

\begin{tabular}{|c|c|c|}
\hline plane & $2 \theta$ & $I_{\text {var }}$ \\
\hline$[111]$ & 30.085 & 100 \\
\hline$[200]$ & 34.868 & 27.03 \\
\hline$[220]$ & 50.138 & 68.79 \\
\hline$[311]$ & 59.599 & 53.49 \\
\hline
\end{tabular}

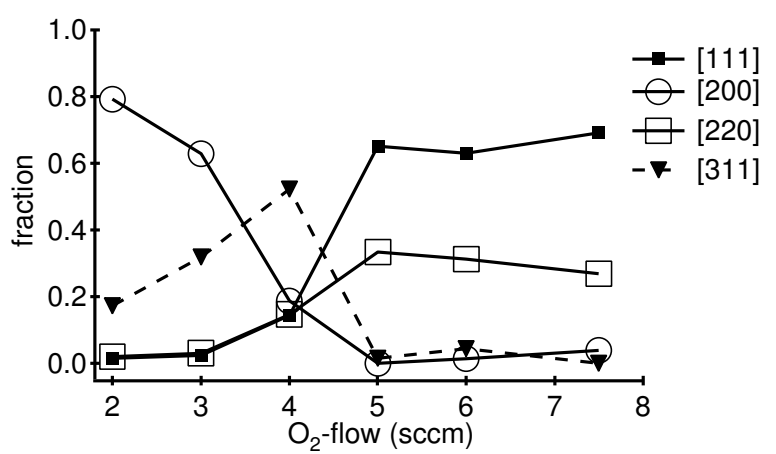

Figure V.3.5: Normalized fraction of grains with a specific out-of-plane orientation as a function of the $\mathrm{O}_{2}$-flow, calculated from the spectra in figure V.3.3.

As well as in figure V.3.3 as in figure V.3.5 it is observed that the out-of-plane alignment changes from [200] to a mixture of 75\% [111] and 25\% [220] when increasing the $\mathrm{O}_{2}$-flow from 2 to $7.5 \mathrm{sccm}$. A considerable fraction of [311] out-of-plane oriented grains is noticed in the transition region, i.e. 3 à 4 sccm $\mathrm{O}_{2}$.

The microstructure of YSZ films deposited at 2 and at $6 \mathrm{sccm}$ was examined by SEM and TEM. Figure V.3.6 shows a TEM cross-section of an YSZ film deposited at $2 \mathrm{sccm} \mathrm{O} \mathrm{O}_{2}$ on glass. Part $\mathrm{a}$ and $\mathrm{b}$ show the microstructure of the lower and upper few hundred nanometers of the YSZ films. It is observed that the film consists of columns, initially expanding and thus overgrowing each other. The column tops are also clearly faceted. Part $\mathrm{c}$ and d show SAED patterns of the bottom and the top of the YSZ film respectively. It is observed that the bottom of the YSZ films is more or less random out-of-plane oriented because of the presence of 
diffraction rings. However, the SAED pattern of the upper part shows clear diffraction spots, corresponding with a [200] out-of-plane alignment. This evolutionary overgrowth resulting in the development of an out-of-plane alignment and clearly faceted columns corresponds to the microstructure of a deposition in zone T (see table II.5).

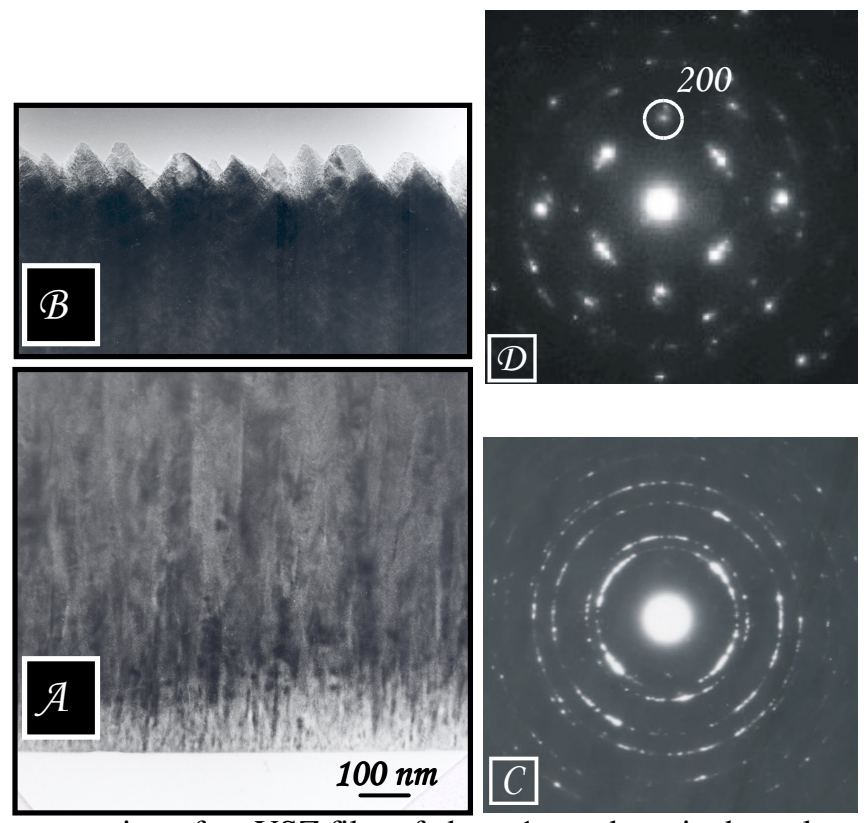

Figure V.3.6: TEM cross-section of an YSZ film of about $1 \mu \mathrm{m}$ deposited on glass at an $\mathrm{O}_{2}$-flow of $2 \mathrm{sccm}$. Part $\mathrm{a}$ and $\mathrm{b}$ show the microstructure of the lower and upper few hundred nanometres of the YSZ film, respectively. In part a, the evolutionary overgrowth resulting in expanding columns is observed. In part $b$, a clear faceting of the column tops is noticed. Part $\mathrm{c}$ and $\mathrm{d}$ are SAED patterns of the lower and upper part of the YSZ film and indicate an evolution from a random oriented to a clear [200] out-of-plane aligned film.

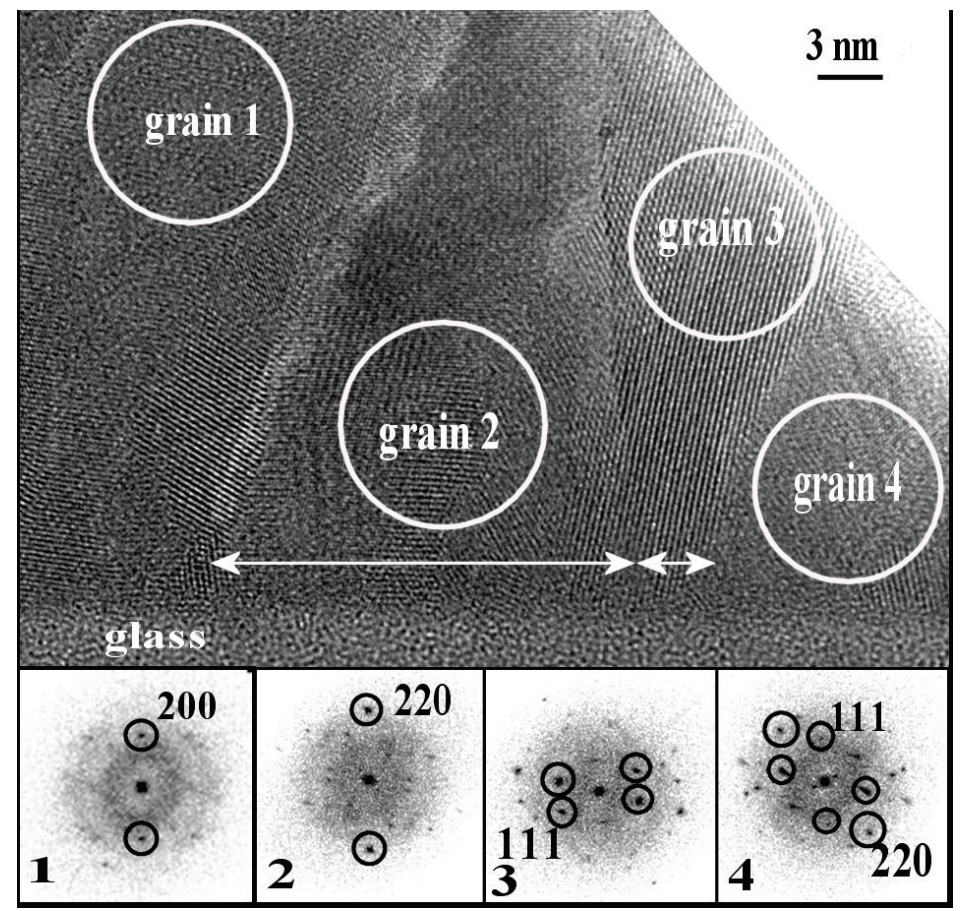

Figure V.3.7: A high resolution TEM picture of the bottom of an YSZ film deposited on glass at an $\mathrm{O}_{2}$-flow of 2 sccm and a target-substrate tilt angle of $55^{\circ}$, with the corresponding Fourier transform calculated patterns. The areas where the Fourier transforms are taken from, are indicated by a white circle. It is clear that the grains with a [200] out-of-plane orientation (grain 1 and 3) overgrow the other grains ([220] and [111] oriented). The white arrows indicate the anisotropy in initial grains size between [200] and other out-of-plane oriented grains. 
Figure V.3.7 shows a high resolution TEM cross-section of the first 35 nanometres of an YSZ film deposited at identical conditions as the above described deposition, but on a tilted substrate $\left(\alpha=55^{\circ}\right)$. However, it will be discussed in section V.7 that, at least for depositions carried out in zone $\mathrm{T}$ conditions, the mechanism of out-of-plane alignment is not really influenced by tilting the substrate. Hence, the observations from this high resolution picture are also valid for a deposition on a non-tilted substrate. In this high resolution picture, four clear grains/columns are noticed. It is observed that two of these grains expand (grain 1 and 3), while two others decrease in size at increasing film thickness. It is also observed that the two expanding grains initially have a smaller grain size compared to the other grains, as indicated by the white arrows. As observed in the corresponding Fourier transform images, the two expanding grains are [200] out-of-plane oriented, while the two other grains are [111] and [220] out-of-plane oriented.

Figure V.3.8 shows a SEM plan view of an YSZ film deposited at $6 \mathrm{sccm} \mathrm{O}_{2}$ on stainless steel. A corresponding TEM cross-section of the same deposition but on glass is shown in figure V.3.9. In fact, the observed microstructure cannot be categorized in one of the described zones in chapter II. No voided, columnar structure is observed. As such, it is not a zone Ia structure. In the cross-section, repeated nucleation is observed, but the structure cannot be a zone Ib or zone Ic structure. This because the resulting out-of-plane orientation is not randomly oriented but consists of 75\% [111] and 25\% [220]. Although some faceting is observed, zone T is excluded since no V-shaped columns are observed. Since neither columns penetrating through the whole film thickness are observed, it is also not a zone II structure.

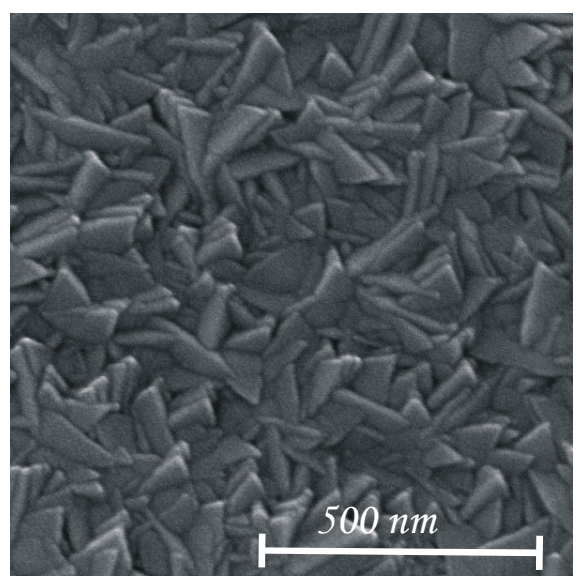

Figure V.3.8: SEM plan view of an YSZ film deposited on stainless steel at $6 \mathrm{sccm} \mathrm{O}_{2}$.

As observed in the SEM plan view, the grains are elongated and reach up to $200 \mathrm{~nm}$ in length. Comparing this with an average column diameter of $40 \mathrm{~nm}$ at a film thickness of $400 \mathrm{~nm}$ for

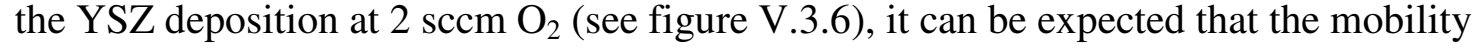
during the thin film deposition is increased. Hence, the resulting microstructure is possibly a combination or mixture of two higher mobility zones. Since it is only in zone T and zone II that a preferential out-of-plane orientation originates during the deposition (see chapter II), it can be concluded that the microstructure must be a mixture of zone T and zone II.

It can be concluded that an increasing reactive gas flow, in case of the YSZ deposition causes: - a transition in microstructure from clear zone T towards a mixture of zone T and zone II, when increasing the $\mathrm{O}_{2}$-flow from 3 to $5 \mathrm{sccm}$.

- a corresponding transition from a [100] out-of-plane alignment to a mixture of $75 \%$ [111] and 25\% [110] out-of-plane oriented grains. 


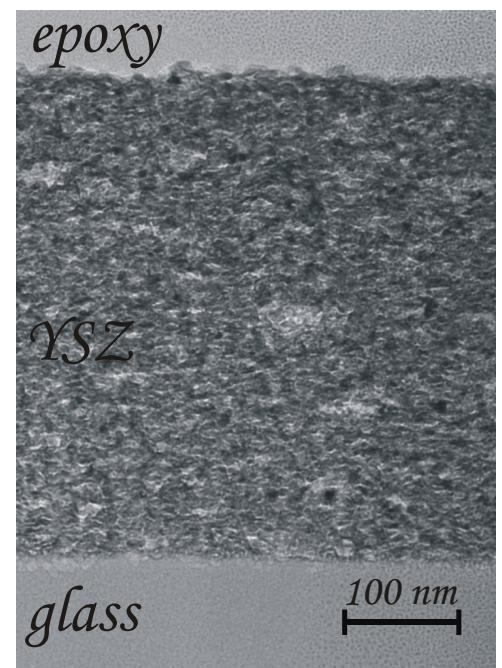

Figure V.3.9: TEM cross-section of an YSZ film deposited at $6 \mathrm{sccm} \mathrm{O}_{2}$ on glass.

Possible influence of impurities:

Although it was described in chapter II (section II.3) that impurities such as hydrocarbons, water vapour or $\mathrm{CO}_{2}$ can have a large influence on the basic structure forming phenomena and thus also on the resulting microstructure, no attention to these impurities was paid up to now. Since it was described that an increasing flux of impurities can alter the resulting preferential out-of-plane orientation, can cause repeated nucleation and can change the grain size, it should be checked if the transition in microstructure and out-of-plane orientation with increasing $\mathrm{O}_{2}$-flow is not a consequence of an increased impurity contribution.

Since the observed transition occurs in the range between 3 and $5 \mathrm{sccm} \mathrm{O}_{2}$, let us compare the two corresponding depositions. Although these depositions are carried out at the same base pressure, at the same pressure $(0.55 \mathrm{~Pa})$ and the same total gas flow $(60 \mathrm{sccm})$, the impurity concentration in the deposited film can increase due to a strongly decreased deposition rate (from 48.8 to $12.8 \mathrm{~nm} / \mathrm{min}$ ) as seen in figure V.3.1. Knowing the purity of the target $(99.9 \%$ ), the purity of the gasses $(99.999 \%)$ and the base pressure $\left(4 * 10^{-4} \mathrm{~Pa}\right)$, the ratio between impinging impurities and deposited metallic particles could be estimated. The total flux of impurities towards the substrate is given by: $\Theta=\frac{4 * 10^{-4}+0.55 * 10^{-5}}{\sqrt{2 \pi M k T}}+\Theta_{M} * 10^{-3}$, in which an average value of 40 a.m.u. for $\mathrm{M}$ is used and in which T is $300 \mathrm{~K} . \Theta_{\mathrm{m}}$ is the flux of metallic particles towards the growing film, which can be calculated from the deposition rate. It was calculated that this ratio $\Theta / \Theta_{M}$ increased by a factor of 3.83 when increasing the $\mathrm{O}_{2-}$ flow from 3 to $5 \mathrm{sccm}$. In order to check whether the observed transition in microstructure and out-of-plane orientation with increasing $\mathrm{O}_{2}$-flow originates from an increased impurity contribution, a series of depositions at different base pressure was carried out. YSZ films of about $1 \mu \mathrm{m}$ were deposited at the above used deposition conditions, with an $\mathrm{O}_{2}$-flow of $3 \mathrm{sccm}$ but with a base pressure from $4 * 10^{-6}$ up to $4.4 * 10^{-5}$ mbar. If the observed transition in microstructure with increasing $\mathrm{O}_{2}$-flow would be caused by an increased impurity contribution, the same transition should be observed when increasing the base pressure with a factor of 3.9, because this corresponds with an increase of the ratio between impinging impurities and deposited metallic particles by a factor of 3.83. As noticed in figure V.3.10, no transition in the out-of-plane alignment could be observed up to a base pressure of $4.4 * 10^{-5}$ mbar. 


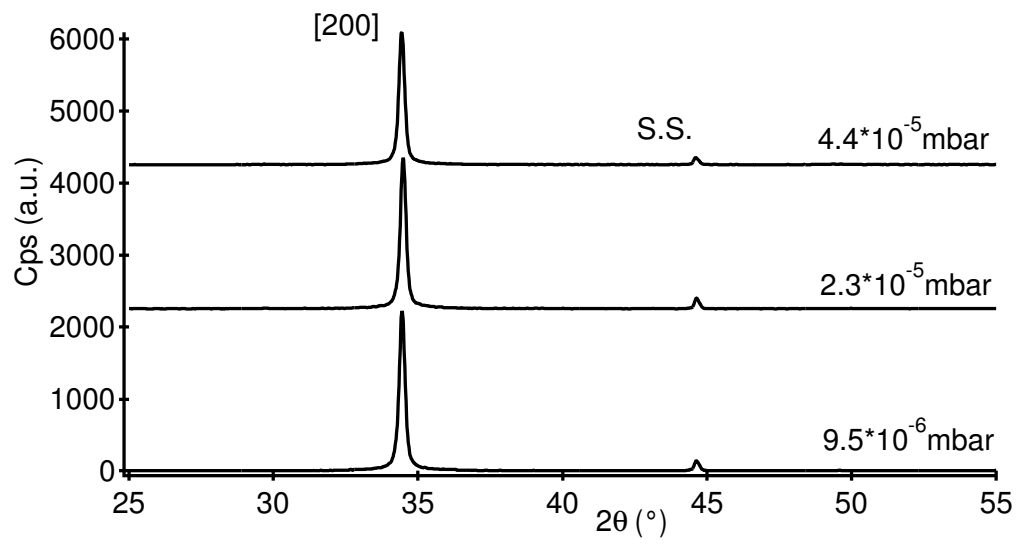

Figure V.3.10: $\theta / 2 \theta$ spectra of YSZ films deposited on stainless steel with an $\mathrm{O}_{2}$-flow of 3 sccm at different base pressures. No clear difference in out-of-plane orientation can be observed.

It can be concluded that the transition in microstructure and out-of-plane orientation when increasing the $\mathrm{O}_{2}$-flow from 3 to 5 sccm is not a consequence of an increased contribution of the impurities.

Similar depositions of TiN were carried out to investigate a possible influence of the base pressure on the microstructure and out-of-plane orientation. Again no variation in microstructure nor in out-of-plane alignment for depositions carried out at the same conditions but with a base pressure varying between $3.2 * 10^{-6}$ and $2 * 10^{-5}$ mbar.

\section{4: Influence of an increasing reactive gas flow: comparison between TiN and YSZ.}

In section V.2 and V.3, the influence of an increasing reactive gas flow on the resulting microstructure and out-of-plane orientation of TiN and YSZ respectively, was described. For both materials, a strong influence was observed. That influence of the reactive gas flow is schematically summarized in table V.4.1.

Table V.4.1: Schematic summary of the influence of an increasing reactive gas flow on the microstructure and out-of-plane orientation of reactive magnetron sputtered TiN and YSZ.

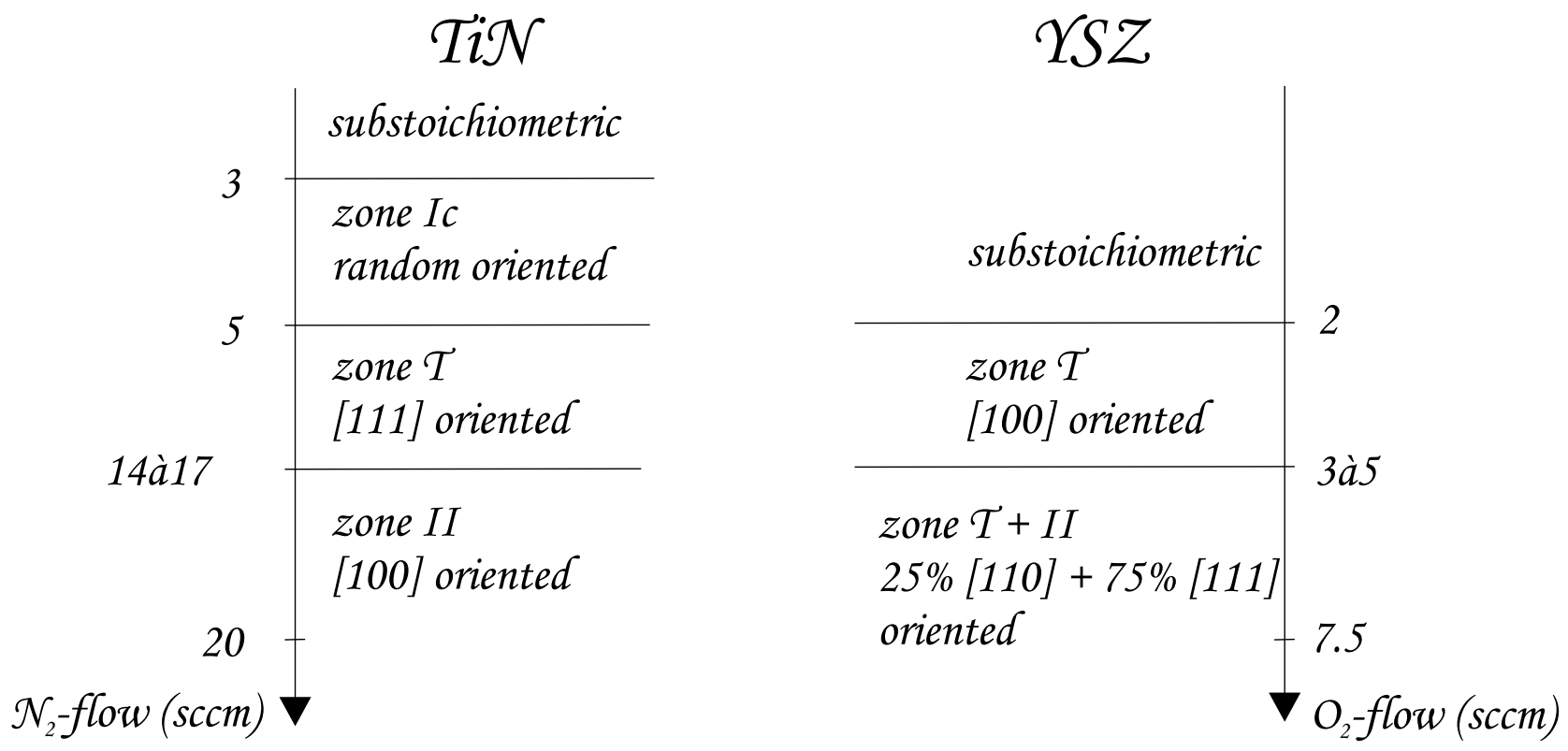




\section{5: Published models for out-of-plane alignment}

As already mentioned in the introduction, not that much models concerning the growth and out-of-plane orientation in sputter deposited thin films have been published in literature. As far as it is known, only three major models discussing the influence of different deposition parameters on the resulting out-of-plane orientation of TiN films were published, while none for YSZ. In this section, these three major models will be discussed and compared to our results.

\section{V.5.1: Overall energy minimization:}

First Pelleg et al.[23] and later Oh and Je [11,12] considered the overall energy of a film as the summation of the strain energy and the surface energy. The total strain energy is expressed by the intrinsic stress $E$ multiplied by the film thickness $h$. Hence, the total strain energy increases with increasing film thickness. Later on, Zhao extended the overall energy model by also taking into account the energy delivered by the impingement of energetic ions on the growing film and called this the stopping energy [10]. Then the overall energy is written as: $W=S+E h+I$, in which $W$ expresses the total energy, $S$ the surface energy, $E$ the intrinsic stress, $h$ the film thickness and $I$ the stopping energy. In case that the mobility during the thin film growth is large enough to aim for the thermodynamic most favourable situation, the film will aim for the lowest overall energy $W$. Thus the growing film not only has a driving force to lower the surface energy, but also other driving forces to minimize the strain energy and the stopping energy. According to Oh, Je and Zhao, the competing planes in the TiN film are the (200) plane with the lowest surface energy, the (111) plane with the lowest strain energy and the (220) plane with the lowest stopping energy. However, it was calculated by Zhao that the stopping energy should only be taken into account when the energy of the bombarding particles exceeds $400 \mathrm{eV}$ [10]. As such, in most conditions the influence of the stopping energy can be ignored.

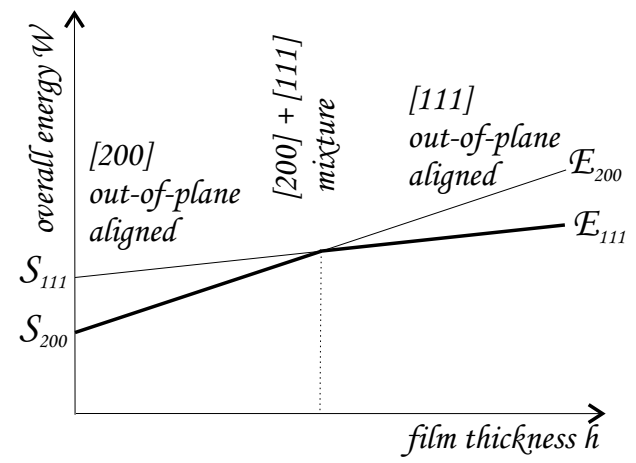

Figure V.5.1: Variation of the overall energy of a TiN film with increasing thickness, for a film grown without high energetic $(>400 \mathrm{eV})$ bombardment.

According to this model, a TiN film should have a [200] out-of-plane alignment as long as the strain energy is small. This strain energy increases with increasing film thickness, and thus exceeding a critical thickness a [111] out-of-plane alignment should develop. If the film is bombarded by particles of at least $400 \mathrm{eV}$, a [220] out-of-plane alignment should develop. The variation of the overall energy with increasing film thickness, ignoring the influence of the stopping energy is shown in figure V.5.1.

In case of YSZ, the plane of lowest surface energy is the (111) plane [2]. However, there is still discussion in literature which crystallographic plane has the lowest stopping energy. Iijima [24] stated that the [100] direction offers the lowest stopping energy. However, Ressler [25] measured that it was the [111] direction, while Dong [26] calculated that the [110] 
direction should offer the lowest stopping energy. Therefore, no conclusions about the influence of the stopping energy on the resulting out-of-plane orientation can be drawn. The same is true about the influence of the stress energy, since no publications about the anisotropy in stress energy for an YSZ structure could be found in literature. It can be concluded that the influence of an overall energy minimization on the resulting out-of-plane orientation of YSZ can't be discussed, due to the lack of information.

Trying to convince the reader that stress is not the determining factor for the change from a [111] to a [100] out-of-plane alignment in TiN, several reasons and arguments are given in order to show that this overall energy model is not correct.

- Abadias [13] studied by means of XRD the residual stress in preferential oriented TiN films grown by sputter deposition. Stress analysis of TiN films having a mixed [200] and [111] out-of-plane orientation indicated that the [111] out-of-plane oriented grains are more stressed than the [200] oriented grains. Since the overall energy model explains the transition from a [200] to a [111] out-of-plane alignment in order to minimize the strain energy, it is in contradiction with the results of Abadias. More precisely, according to Abadias changing to a [111] out-of-plane alignment would only increase the strain energy. Also Petrov et al. observed for a TiN film with mixed out-ofplane orientation that the [111] oriented grains are more stressed than the [200] out-ofplane oriented grains [27].

- Pelleg et al. [23] calculated that a critical film thickness should exist. For lower thickness, the TiN film should have a [200] out-of-plane alignment, while for higher thickness the film should be [111] out-of-plane aligned. Pelleg calculated that this critical thickness should be at maximum $1.5 \mu \mathrm{m}$, depending on the used deposition conditions. However, all films described in section V.2 have nearly the same thickness of $1 \mu \mathrm{m}$. Hence, the variation from [200] to [111] could not be explained by exceeding a critical thickness. Moreover, [200] out-of-plane aligned films of more than $2 \mu \mathrm{m}$ have been deposited at the same deposition conditions as used in section V.2. As such, no evidence for a critical film thickness was found.

- According to the overall energy model, a variation in the strain energy when changing the out-of-plane orientation should be observed. A measure for this strain energy is obtained by calculating the dimension of the unit cell $a$ from the observed peak positions in a $\theta / 2 \theta$ spectrum. As observed in figure V.2.2, the peak positions of the [111] out-of-plane aligned films are not shifted with respect to the peak positions of the [200] out-of-plane aligned films.

- In the same way Greene et al. [16] calculated the strain energy of [111] and [200] out of-plane aligned films and did also not observe a systematic peak shift.

- Even if the overall energy model would be correct, and if the strain energy of the [111] direction would be lower than the strain energy of the [200] direction, the observations of $\mathrm{Oh}$ and $\mathrm{Je}[11,12]$ themselves are in contradiction with the model. More precisely, assuming that the film is able to aim for the thermodynamic most favourable situation (i.e. lowest energy) implies that the mobility during the growth is high enough to allow re-structurative grain growth (see chapter I and II). Hence, if a film would have a [111] out-of-plane alignment due to the drive for the lowest strain energy, no [200] out-ofplane oriented grains may be observed at the bottom of the film. This because these [200] grains should be consumed by the [111] grains during the re-structuring. However, Oh observed [200] out-of-plane oriented grains at the bottom of a [111] outof-plane aligned film by electron diffraction, indicating that no re-structurative growth occurred [11]. 
Consequently, it is believed that the observed transition from random oriented to a [111] and finally to a [200] out-of-plane alignment in TiN films as described in section V.2 cannot be explained by the overall energy model. Also the YSZ observations can't be explained by the overall energy model since not enough data about the energy due to stress and ion bombardment is known.

\section{V.5.2: Energetic bombardement effect:}

Several authors observed a transition in preferential orientation when increasing the energy of the perpendicular incident particles.

- In case of TiN, a transition from a clear [111] out-of-plane alignment to a more random or even [200] out-of-plane alignment when increasing the bombarding particle energy [4, 15, 1921, 27,28]. Only Zhao [10] observed for TiN a transition from [111] to a [220] out-of-plane alignment. It was observed that a directed beam of particles with an energy of at least $100 \mathrm{eV}$ is needed to disturb the [111] out-of-plane alignment. Moreover, a [200] out-of-plane alignment (or [220] in case of Zhao) could only be obtained when the energy was increased up to a few hundred eV.

All these authors explain the transition of the out-of-plane alignment by the anisotropy of the collision effect. It was argued that the ion energy in open channelling directions is distributed over large depths, giving rise to less lattice distortion and lower sputter yields. In this way, planes orthogonal to open channelling directions have a higher probability of surviving and will overgrow the other grains. It was calculated that in case of a rocksalt structure, the [111] direction exhibit the densest array of atom columns, while the [200] direction is the most open channelling direction. Based on an analogous reasoning, Zhao et al. could explain the transition to a [220] out-of-plane alignment by assuming that TiN has an fcc structure, although TiN has a rocksalt structure.

- In case of YSZ, Iijima [25] stated that the [100] direction is the best channelling direction. However, Ressler [26] measured that the [111] direction has got the lowest re-sputter rate, while Dong [28] calculated that the [110] direction should be the best channelling direction. Hence, it isn't known how an energetic bombardment influences the resulting out-of-plane orientation of YSZ films.

There are three possible sources for such an energetic bombardment on the growing film: accelerated ions by the potential difference $\left|V_{p^{-}} V_{s}\right|$, reflected and neutralized ions from the target and ambipolar diffusion. All three contributions are discussed.

- Ions accelerated by $\mid V_{p} \underline{\underline{\underline{S}}} \underline{\underline{\underline{S}}} \underline{\text { : }}$

The TiN and YSZ depositions described in V.2 and V.3 are carried out without an additional ion source and on a grounded substrate $\left(V_{s}=0\right)$. Thus, the positive ions from the plasma are only accelerated by the potential difference between the plasma and the substrate $\left|V_{p}-V_{s}\right|=V_{p}$.

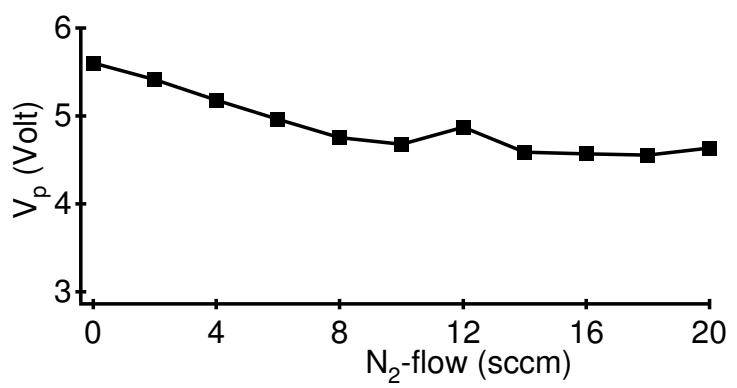

Figure V.5.2: Influence of the $\mathrm{N}_{2}$-flow on the plasma potential $V_{p}$ during the sputtering of a Ti target at $0.55 \mathrm{~Pa}$ at T-S distance of $13 \mathrm{~cm}$ and $I_{d}$ of $0.9 \mathrm{~A}$ as measured with a cylindrical Langmuir probe. 
As shown in figure V.5.2. the plasma potential $V_{p}$ did not really change with increasing $\mathrm{N}_{2}-$ flow during the TiN depositions and remained $5 \pm 0.5 \mathrm{~V}$ as measured by a cylindrical Langmuir probe at the used deposition conditions.

Similar for the YSZ depositions, the plasma potential was measured by means of the Langmuir probe at the used deposition conditions. The influence of an increasing $\mathrm{O}_{2}$-flow on the plasma potential $V_{p}$ is shown in figure V.5.3. It is observed that increasing the $\mathrm{O}_{2}$-flow above $1.8 \mathrm{sccm}$, the plasma potential drops to $-10 \mathrm{~V}$. Such a drop to a negative plasma potential in the vicinity of the substrate has also been reported in literature [29] and is explained by a disappearing anode effect. The chamber walls and substrate disappear as anode because at an $\mathrm{O}_{2}$-flow higher than $1.8 \mathrm{sccm}$ electrically insulating YSZ is coated on the chamber walls and on the substrate. This causes the potential of the substrate and the chamber walls to decrease from grounded to floating. As such, the chamber walls and substrate are not longer an effective anode, causing a drop in the potential of the plasma in front of them. Hence, the drop in the plasma potential is caused by a drop of the substrate potential from grounded to floating at $1.8 \mathrm{sccm} \mathrm{O}_{2}$. Consequently, the potential difference between substrate and plasma $\left|V_{p^{-}} V_{s}\right|$ changes from 5 to 15 when exceeding $1.8 \mathrm{sccm} \mathrm{O} \mathrm{O}_{2}$ as the floating potential is $-25 \mathrm{~V}$.

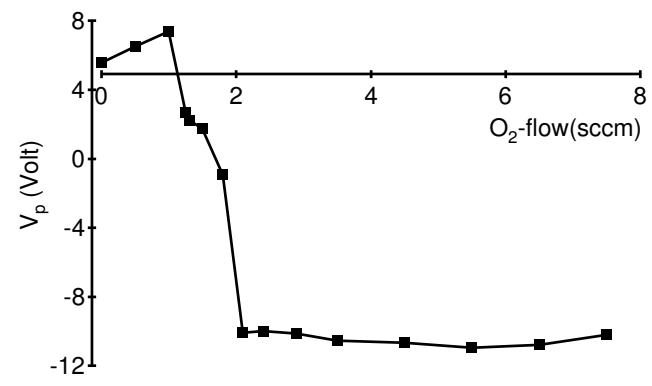

Figure V.5.3: The plasma potential $V_{p}$ as a function of the $\mathrm{O}_{2}$-flow during the sputtering of a $\mathrm{Zr} / \mathrm{Y}$ target at $I_{d}=$ $0.9 \mathrm{~A}, \mathrm{P}=0.5 \mathrm{~Pa}, \mathrm{~T}-\mathrm{S}$ distance of $13 \mathrm{~cm}$.

It can be concluded that in case of the TiN depositions, the positive ions are only accelerated by $5 \mathrm{~V}$ towards the substrate, while in case of the YSZ depositions, the positive ions are accelerated by 5 or $15 \mathrm{~V}$ towards the growing film, depending on the $\mathrm{O}_{2}$-flow. In both cases, no high energetic flux $(>100 \mathrm{eV})$ towards the film is caused by a potential difference between the plasma and the substrate: $\left|V_{p^{-}} V_{s}\right|$.

- Ambipolar diffusion:

This ambipolar diffusion results from the difference in mobility of the electrons and ions. As discussed in chapter III, our depositions are carried out with an unbalanced magnetron type II. The use of such a magnetron causes a high density plasma in the vicinity of the target (cathode) and a lower density plasma further away from the target up to the substrate. Due to this variation in plasma density there is a gradient in the electron and ion density or concentration. However, if there is a concentration gradient of particles, a net flow of these particles down to the gradient results. This is the phenomenon of diffusion. Since the diffusion coefficients of the particles are related to their mobility, the outward diffusion of the electrons will be much faster than the diffusion of the ions. A flow of electrons can also be established without a difference in plasma density. In this later case, the flow of electrons is caused by a gradient in the magnetic field strength. Due to the specific electromagnetic field used in an unbalanced magnetron, this diffusion of the electrons will mainly happen parallel to the magnetic field lines away from the target, in the direction to the substrate. Hence, initially a stream of electrons to the substrate sets in. But this directed flow of the electrons leaves the rest of the plasma more positive and sets up a restraining field which grows large 
enough to equalize the diffusion rates of the ions and electrons. In this way, a flux of accelerated ions following the diffusing electrons arises and a possible beam of energetic ions towards the substrate results [30].

In order to check if the transition in out-of-plane alignment for the TiN and YSZ depositions as mentioned in section V.2. and V.3 originates from an energetic bombardment effect due to ambipolar diffusion or reflected ions, energy resolved mass spectroscopy was carried out. Such an energy resolved mass spectrometer (type PPM 422 of Pfeiffer- Vacuum) is able to measure the energy distribution of ions with a specific mass per charge unit. Since the depositions are carried out in a mixture of $\mathrm{Ar} / \mathrm{N}_{2}$ or $\mathrm{Ar} / \mathrm{O}_{2}$ the energy of particles with a mass per charge unit of 36, 32, 28 and 14 are measured. In this way, the energy distributions of $\mathrm{Ar}^{+}, \mathrm{O}_{2}{ }^{+}, \mathrm{N}_{2}{ }^{+}$and $\mathrm{N}^{+}$are measured, assuming that the particles are single charged positive. The Ar 36 isotope is used since the detector is in saturation when measuring the Ar 40 peak. These energy distributions during the TiN depositions were measured at a target-substrate distance of $13 \mathrm{~cm}$, a pressure of $0.55 \mathrm{~Pa}$, a discharge current $I_{d}$ of $0.9 \mathrm{~A}$ and an $\mathrm{Ar} / \mathrm{N}_{2}$ flow ratio of $60 / 0$, $50 / 10$ and 40/20. The results of these measurements are shown in figure V.5.4.
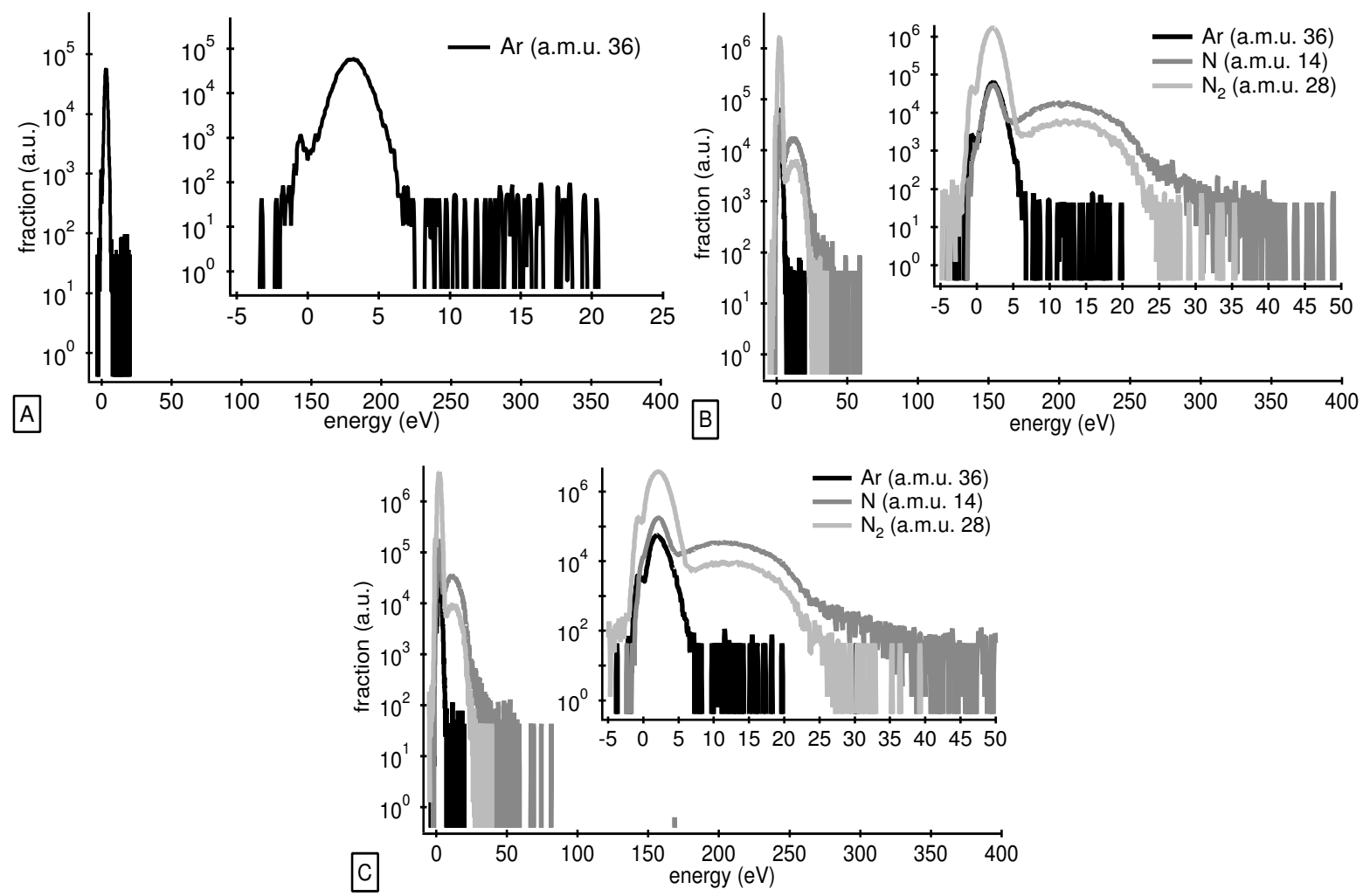

Figure V.5.4: Energy distributions of $\mathrm{Ar}^{+}, \mathrm{N}^{+}$and $\mathrm{N}_{2}{ }^{+}$ions measured by an energy resolved mass spectrometer for a discharge in an $\mathrm{Ar} / \mathrm{N}_{2}$ flow ratio of 60/0 (a), 50/10 (b) and 40/20 (c). In case of an $\mathrm{Ar} / \mathrm{N}_{2}$ flow ratio of 60/0 only the $\mathrm{Ar}^{+}$ions were measured. In the inset, a magnification of the lower energy part is shown.

No real difference between the energy distributions is measured for an $\mathrm{Ar} / \mathrm{N}_{2}$ flow ratio of $50 / 10$ and 40/20, indicating that the transition from [111] to [200] out-of-plane alignment is not caused by an increased high energetic ion bombardment, when increasing the $\mathrm{N}_{2}$-flow from 5 to $20 \mathrm{sccm}$.

Beside no clear variation of the energy distributions when increasing the $\mathrm{N}_{2}$-flow, it is also observed that no directed beam of high energetic ions $(>100 \mathrm{eV})$ reaches the substrate for all investigated $\mathrm{Ar} / \mathrm{N}_{2}$ flow ratios. The mean energy of the ions is $\sim 5 \mathrm{eV}$, corresponding with the plasma potential $V_{p}$ as measured with the Langmuir probe (see fig. V.5.2). In case of the $\mathrm{N}^{+}$ and $\mathrm{N}_{2}{ }^{+}$energy distributions, a fraction of ions with an energy of $\sim 15 \mathrm{eV}$ is observed. In case of $\mathrm{N}_{2}{ }^{+}$this fraction is about 2 à 3 orders of magnitude lower than the intensity of the peak at 5 
$\mathrm{eV}$, while in case of $\mathrm{N}^{+}$this fraction is only less than 1 order of magnitude smaller than the intensity of the peak at $5 \mathrm{eV}$. As will be discussed below, this additional peak at $\sim 15 \mathrm{eV}$ is possibly a result of the reflected ions at the target.

Also the energy of the $\mathrm{Ar}^{+}$and $\mathrm{O}_{2}{ }^{+}$ions during the $\mathrm{YSZ}$ deposition was measured by means of energy resolved mass spectroscopy. The results are shown in figure V.5.5. As observed in these energy distributions, no high energetic $(>100 \mathrm{eV})$ beam of ions was observed, neither for the deposition at $1 \mathrm{sccm} \mathrm{O}_{2}$, nor for the deposition at $6 \mathrm{sccm} \mathrm{O}_{2}$. It is also observed that the peak maximum shifts from about $+2 \mathrm{~V}$ to $-13 \mathrm{~V}$ when increasing the $\mathrm{O}_{2}$-flow from 1 to 6 sccm. This corresponds with the drop of the plasma potential as shown in figure V.5.3. Again, it is noticed that no beam of high energetic ions reaches the growing film.
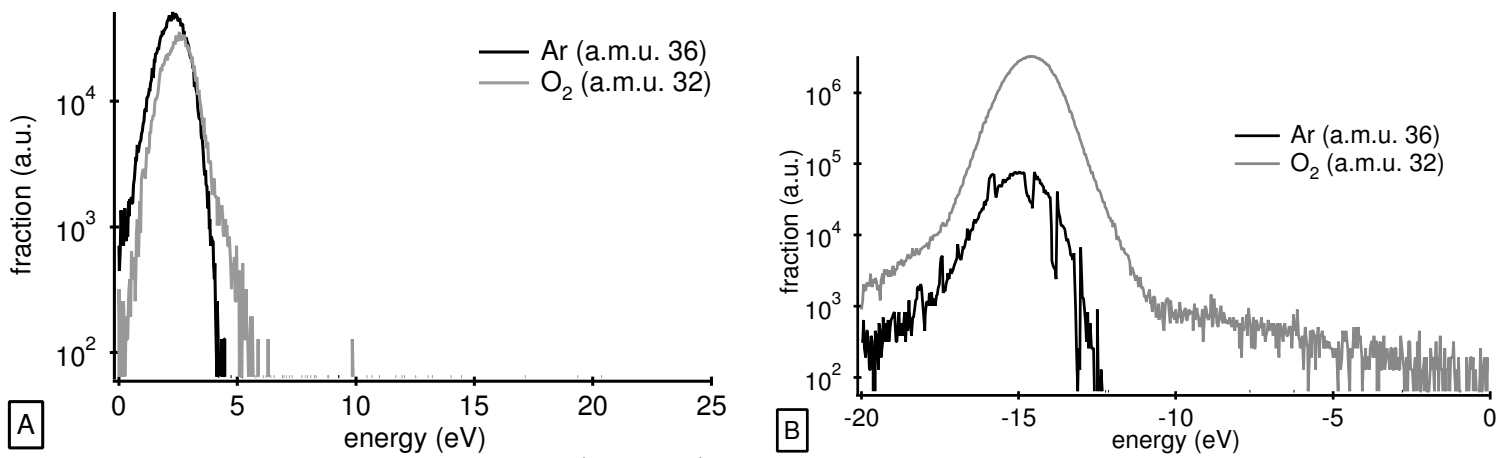

Figure V.5.5: Energy distributions of $\mathrm{Ar}^{+}$and $\mathrm{O}_{2}{ }^{+}$ions measured by an energy resolved mass spectrometer for a discharge in an $\mathrm{Ar} / \mathrm{O}_{2}$ flow ratio of 59/1 (a) and 54/6 (b).

- reflected and neutralized ions:

According to the above described energetic bombardment effect, not only energetic ions but also energetic neutrals bombarding the growing film may cause a transition in out-of-plane alignment. In case of magnetron sputtering, such energetic neutrals can originate from reflected ions at the target. $\mathrm{Ar}^{+}, \mathrm{O}_{2}{ }^{+}$and $\mathrm{N}_{2}{ }^{+}$ions are accelerated over the potential difference $\left|V_{p^{-}} V_{d}\right|$ towards the target. At the target, these ions can cause a collision cascade and get incorporated in the target but can also be reflected. If these reflected particles are in the neutral state they are not influenced by the electric field and may reach the substrate with a considerable energy. Some of these reflected particles can get ionized in the plasma and hence can be measured by the energy resolved mass spectrometer. However, it is not straightforward to measure the energy distribution of the reflected particles that remained in the neutral state. Therefore, the energy distributions of the reflected ions at the target were calculated by SRIM [31]. As seen in figure V.5.6. the energy distributions of the reflected particles during the TiN deposition don't show a beam of high energetic $(>100 \mathrm{eV})$ particles. Figure V.5.7 shows similar results for the reflected particles during the depositions of YSZ.

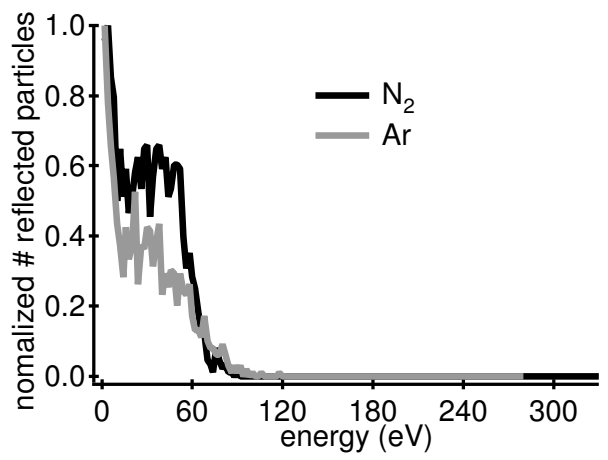

Figure V.5.6: Calculated energy distribution of reflected $\mathrm{Ar}^{+}$and $\mathrm{N}^{+}$ions at a Ti target, assuming a potential difference $\left|\mathrm{V}_{\mathrm{p}}-\mathrm{V}_{\mathrm{d}}\right|$ of $330 \mathrm{eV}$ ( or $165 \mathrm{eV}$ in case of $\mathrm{N}$ ). 
Since it was observed $[4,10,15,19-21,27,28]$ that a bombardment of particles of several hundreds of eV is needed to change the out-of-plane orientation from [111] to [100], the ion reflection at the target can't explain the transition in out-of-plane alignment.

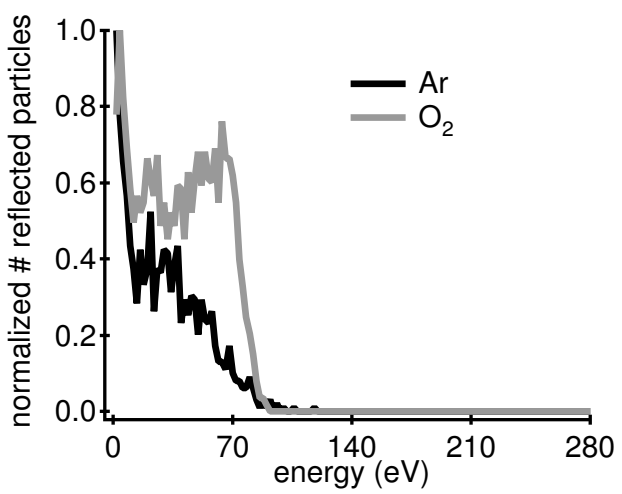

Figure V.5.7: Calculated energy distribution of reflected $\mathrm{Ar}^{+}$and $\mathrm{O}^{+}$ions at a $\mathrm{Zr} / \mathrm{Y}$ target, assuming a potential difference $\left|\mathrm{V}_{\mathrm{p}}-\mathrm{V}_{\mathrm{d}}\right|$ of $280 \mathrm{eV}$ (or $140 \mathrm{eV}$ in case of $\mathrm{O}$ ).

It is also observed in figure V.5.6 that a considerable fraction of the reflected N-particles has an energy up to $60 \mathrm{eV}$. These higher energy $\mathrm{N}$-particles may cause the higher energy ions $(\sim 15 \mathrm{eV})$ as measured with the energy resolved mass spectrometer (see fig. V.3.3). In fact these reflected $\mathrm{N}$-particles originated from accelerated $\mathrm{N}_{2}$-ions which has dissociated by impact on the target.

It can be concluded that there is no real beam of energetic particles towards the substrate due to the $\left|V_{p}-V_{s}\right|$ difference, due to ambipolar diffusion nor due to reflected and neutralized ions. Therefore, the energetic bombardment effect can't explain the observed transition in out-ofplane alignment for TiN and YSZ as observed in section V.2 and V.3.

\section{V.5.3: Kinetics versus thermodynamics:}

In the previous section, it was discussed that the microstructure of the deposited TiN films changed from a zone Ic to a zone $\mathrm{T}$ and finally into a zone II structure with increasing $\mathrm{N}_{2^{-}}$ flow, while the microstructure changed from zone $\mathrm{T}$ to a mixture of zone $\mathrm{T}$ and zone II in case of YSZ. As discussed in chapter II, such transitions should correspond with an increasing mobility during the thin film growth. Langmuir probe, energy resolved mass spectroscopy, transport simulations and deposition rate measurements were carried out in order to find out how an increasing reactive gas flow could increase the mobility during the film deposition. It has been described that the mobility during the thin film growth mainly originates from the energy flux caused by [32-35]:

- substrate heating

- energy of sputtered metallic adparticles

- release of energy during the formation of a compound at the substrate, i.e. condensation energy

- plasma-surface interactions by the impingement of electrons and ions.

- Substrate heating:

All the depositions were carried out on a non-heated substrate. Hence, the substrate temperature was floating, what means that no energy flux originating from a substrate heater has to be calculated.

- energy of sputtered particles

It was discussed in chapter III that sputtered particles have a considerable energy (some eV) when leaving the target surface. However, before impingement on the growing film, these 
sputtered particles have to move through the plasma or the gas phase. During this transport through the gas phase these sputtered particles may endure several collisions and thus loose energy. The energy with which the particles leave the target and the energy loss during the transport through the gas phase will be simulated in chapter VII. Based on the simulations, carried out for the deposition conditions described in section V.2 and V.3, the average energy of the Ti particles is $8.75 \mathrm{eV}$ and the average energy of the $\mathrm{Zr}$ and $\mathrm{Y}$ particles is $8.65 \mathrm{eV}$ when reaching the growing film, at the used deposition conditions.

\section{- Condensation energy:}

The energy released during the formation of TiN(s) from Ti $(\mathrm{g})$ and $1 / 2 \mathrm{~N}_{2}(\mathrm{~g})$ is $8.4 \mathrm{eV}$ [36]. Assuming that the deposited film is stoichiometric and that all incoming metallic adparticles are $\mathrm{Ti}$, then $8.4 \mathrm{eV}$ per bonded $\mathrm{Ti}$ particle is released into the film. In an analogous way, the energy released during the formation of $\mathrm{ZrO}_{2}$ and $\mathrm{Y}_{2} \mathrm{O}_{3}$ can be calculated [37]. Taking into account that $55 \%$ of the incoming adparticles is $\mathrm{Zr}$ and $45 \% \mathrm{Y}$, it can be calculated that per deposited metallic particle, an energy of $0.71 \times 17.7\left(\mathrm{ZrO}_{2}\right)+0.29 \times 6.79\left(\mathrm{Y}_{2} \mathrm{O}_{3}\right)=14.54 \mathrm{eV}$ is released due to the formation of the compound.

- Electron and ion energy flux:

Finally, the energy flux originating from the plasma-surface interaction has to be calculated. This plasma-surface interaction originates mainly from the impingement of electrons and ions.

The electron density $N_{e}$, ion density $N_{i}$ and the average electron temperature $T_{e}$ for a discharge at $0.55 \mathrm{~Pa}, I_{d}=0.9 \mathrm{~A}$, at $13 \mathrm{~cm}$ target-substrate distance and with varying $\mathrm{Ar} / \mathrm{N}_{2}$ or $\mathrm{Ar} / \mathrm{O}_{2}$ ratio have been measured by a cylindrical Langmuir probe. The results are shown in figure V.5.8 and V.5.9 respectively. It is observed that an increasing reactive gas flow not really influences these plasma characteristics.

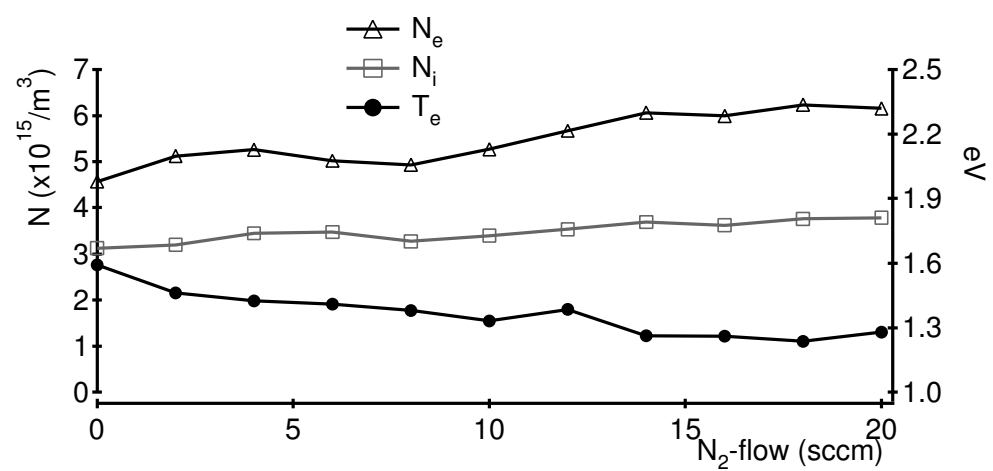

Figure V.5.8: Results of cylindrical Langmuir probe measurements at the same conditions as the depositions of TiN described in section V.2.

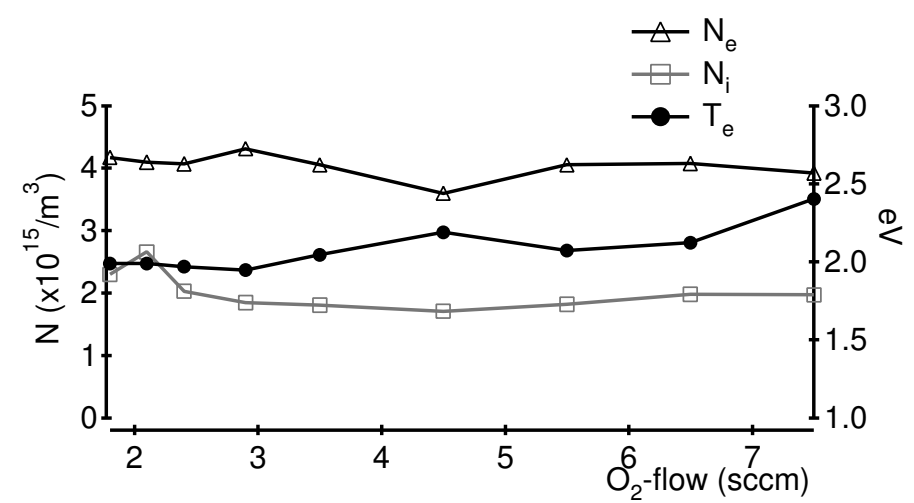

Figure V.5.9: Results of cylindrical Langmuir probe measurements at the same conditions as the depositions of YSZ described in section V.3. 
In order to calculate the electron and ion energy flux towards the substrate, the flux towards the substrate should be multiplied by their energy. However, this flux and energy strongly depends on the potential of the substrate. On the one hand, a substrate with a potential lower than the plasma potential $V_{p}$ (see chapter III) will repel the electrons but will attract the positive ions. Hence, the flux and the energy of the electrons will decrease, while the flux and the energy of the ions will increase. On the other hand, if the substrate is positively biased, it will become the most effective anode of the system and the plasma potential will also increase. This means that a positively biased substrate will repel the ions and attract the electrons. However, in a first approximation the flux and energy of the electrons will not continuously increase since the plasma potential $V_{p}$ also shifts to higher values [38]. Figure V.5.10 schematically shows the influence of a substrate bias on the relative position of the substrate potential $V_{s}$, plasma potential $V_{p}$ and discharge potential $V_{d}$.

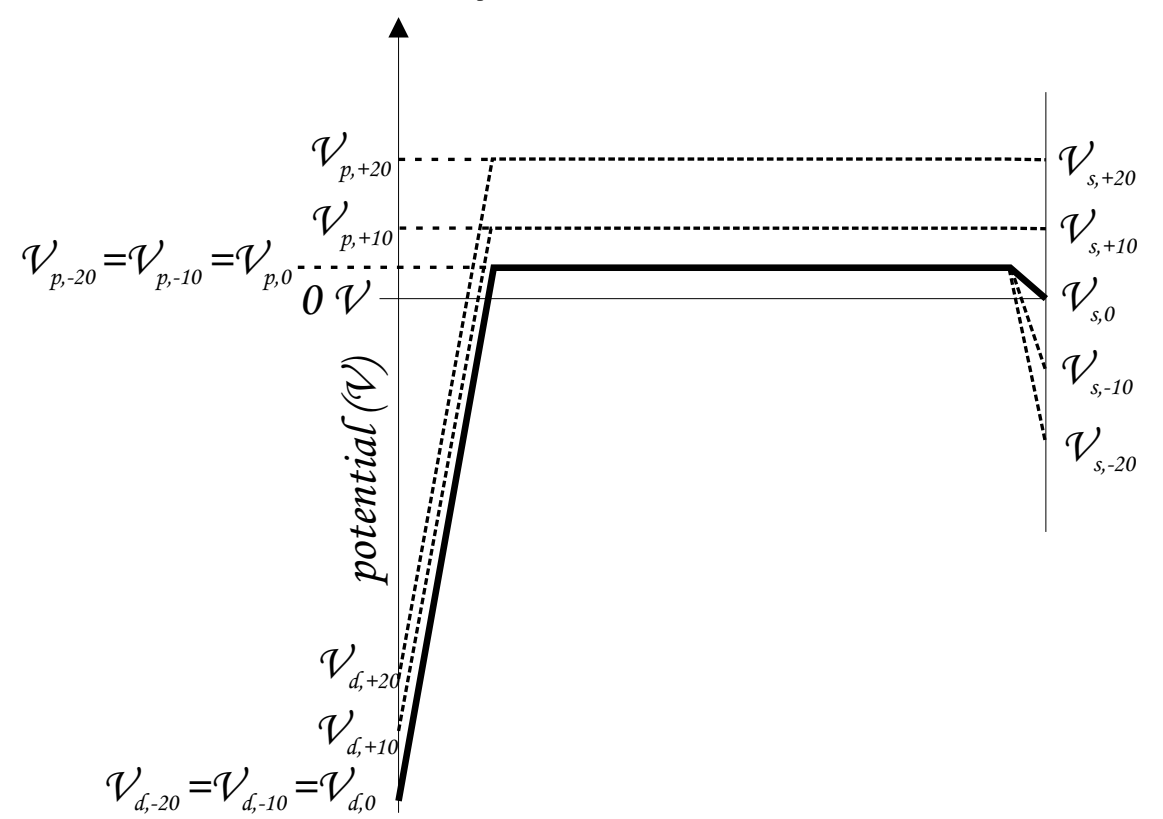

Figure V.5.10: Influence of a substrate bias on the plasma potential $V_{p}$ and discharge potential $V_{d}$. The subscript indicates the bias applied on the substrate with respect to the ground potential.

The depositions are carried out with an unbalanced magnetron. As described in chapter III, a substrate in front of the target will be localized in the plasma plume. Due to the use of an unbalanced type II magnetron and the localisation of the substrate in the dens plasma plume, it can be assumed that the mean energy of the ions is the same as the mean energy of the electrons. As a first approximation, the ions may be regarded as mono-energetic, while the energy of the electrons will have a Maxwell-Boltzmann distribution. Assuming these energy distributions, knowing the electron and ion density $N_{e}$ and $N_{i}$, the average electron temperature $T_{e}$, and taking into account the shift of the plasma potential when biasing the substrate, the electron and ion energy flux towards the substrate can be calculated. The calculation is described in the appendix of this chapter. The result of this calculation is shown in figure V.5.11 for the TiN situation.

The electron and ion energy flux as a function of the potential difference $\left(V_{s^{-}} V_{p, 0}\right)$ are shown, in which $V_{p, 0}$ is the plasma potential in case of a grounded substrate. Since it is observed in figure V.5.8 that an increasing $\mathrm{N}_{2}$-flow not strongly influences the plasma characteristics $N_{e}$, $N_{i}$ and $T_{e}$ average values of $5 \times 10^{15} / \mathrm{cm}^{3}, 3 \times 10^{15} / \mathrm{cm}^{3}$ and $1.5 \mathrm{eV}$ respectively, were used. As such, the energy fluxes shown in figure V.5.11 are in a first approximation valid for an $\mathrm{N}_{2}$ flow between 0 and $20 \mathrm{sccm}$. For the YSZ situation, a similar trend of the energy flux towards the growing film in function of an applied substrate bias can be calculated, simply by changing the values of $N_{e}, N_{i}$ and $T_{e}$. 


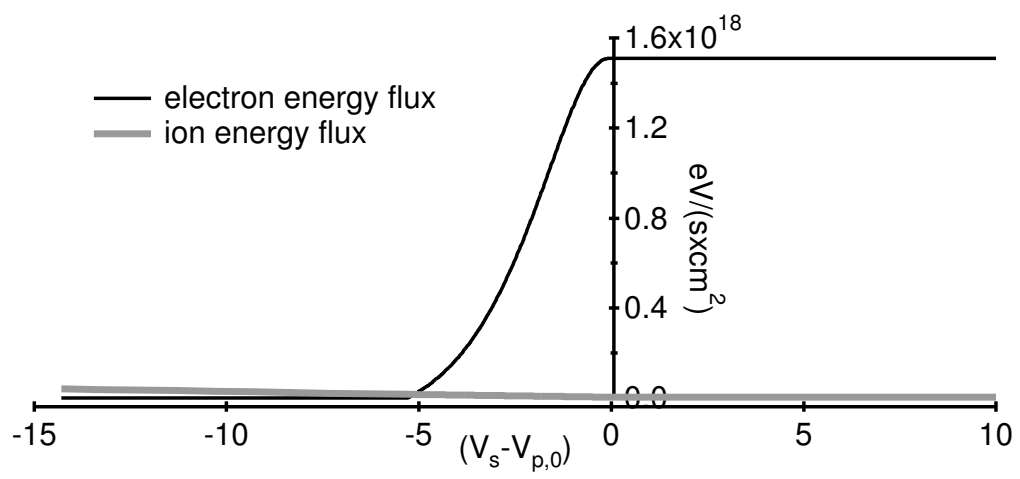

Figure V.5.11: The electron and ion energy flux as a function of the potential difference $\left(V_{s}-V_{p, 0}\right)$, in case of sputtering from a Ti target with an unbalanced magnetron at $0.55 \mathrm{~Pa}, 13 \mathrm{~cm}$ target-substrate distance and $I_{d}$ of 0.9 A.

Total energy flux:

Finally the total energy flux towards the substrate can be calculated. The results are shown in figure V.5.12 and V.5.13 for a deposition of TiN or YSZ at a substrate at $13 \mathrm{~cm}$ distance, at $0.55 \mathrm{~Pa}$ and a discharge current of $0.9 \mathrm{~A}$ using an unbalanced magnetron type II as a function of the $\mathrm{N}_{2}$-flow and $\mathrm{O}_{2}$-flow respectively.

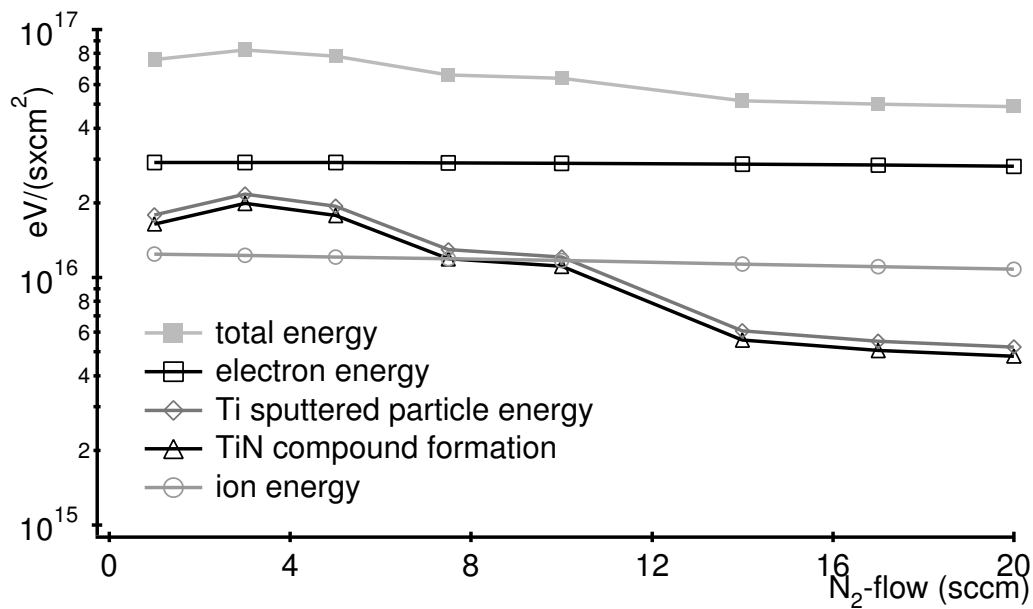

Figure V.5.12: The energy flux towards the substrate due to the energy of the sputtered particles, the formation of TiN compound, the electron-surface interaction and the ion-surface interaction as a function of the $\mathrm{N}_{2}$-flow, assuming $\left(\mathrm{V}_{\mathrm{s}}-\mathrm{V}_{\mathrm{p}}\right)=-5 \mathrm{~V}$. Also the total energy flux is shown.

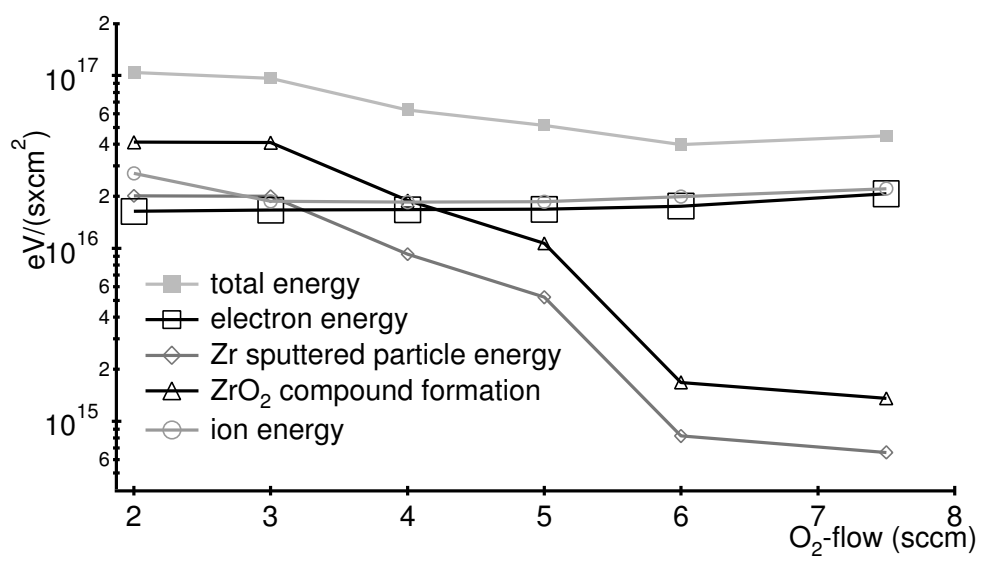

Figure V.5.13: The energy flux towards the substrate due to the energy of the sputtered particles, the formation of YSZ compound, the electron-surface interaction and the ion-surface interaction as a function of the $\mathrm{O}_{2}$-flow assuming $\left(\mathrm{V}_{\mathrm{s}}-\mathrm{V}_{\mathrm{p}}\right)=-15 \mathrm{~V}$. Also the total energy flux is shown. 
To have a measure for the mobility during the growth, the total energy flux should be scaled to the flux of impinging metallic particles. This flux of metallic particles was calculated from the deposition rate, assuming a sticking coefficient of one. The energy flux per deposited $\mathrm{Ti}$ particle in function of the $\mathrm{N}_{2}$-flow for the used deposition conditions is shown in figure V.5.14. Figure V.5.15 shows the energy flux towards the substrate during the YSZ deposition per deposited metallic particle as a function of the $\mathrm{O}_{2}$-flow.

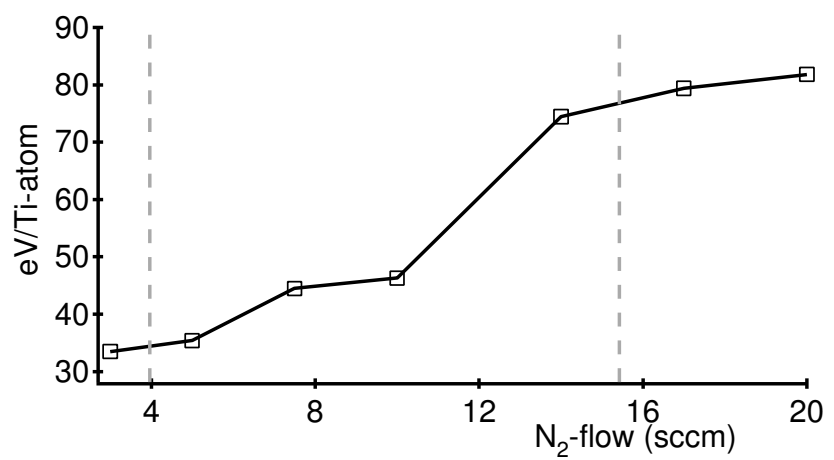

Figure V.5.14: The total energy flux to the substrate per deposited Ti atom as a function of the $\mathrm{N}_{2}$-flow.

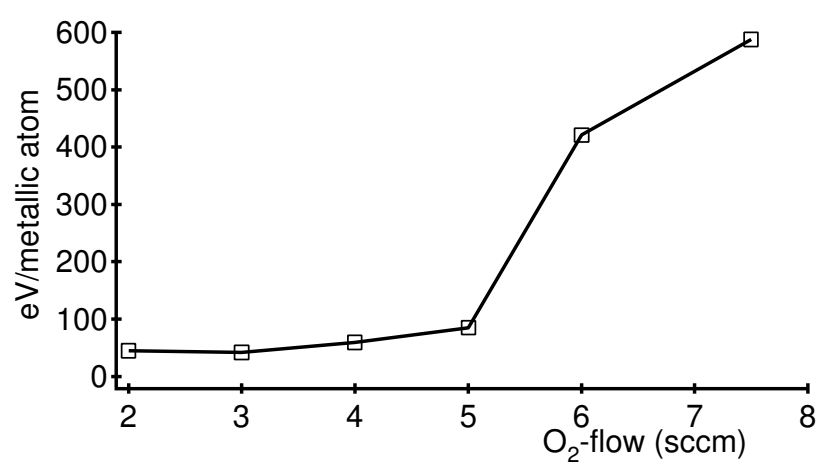

Figure V.5.15: The total energy flux to the substrate per deposited metallic particle as a function of the $\mathrm{O}_{2}$-flow.

It is clearly observed that the energy flux and thus also the mobility increases with increasing reactive gas flow for both materials. It can be concluded that the observed transition from zone Ic, to zone T and finally zone II in case of TiN and the transition from zone T to a mixture of zone T and zone II in case of YSZ is in accordance with the observed increase in energy flux per deposited particle.

As was discussed in chapter II, re-structurative growth may happen if the mobility during the growth is high enough. This re-structurative growth will lead to a crystallographic selection and an out-of-plane alignment. As discussed in the overall energy model, the entire film will aim for its energetically most favourable situation. Hence, the out-of-plane alignment is thermodynamically determined and the resulting preferential orientation depends on the relative strength of the stopping, the strain and the surface energy. As already discussed, the influence of the stopping energy can be neglected since no beam of energetic particles is bombarding the growing film. It was also discussed that the strain energy can not cause a transition in preferential out-of-plane orientation since it is in contradiction with the literature. Hence, aiming at the lowest overall energy means that the entire film will strive for the lowest surface energy. According to Zhao, this means that a (200) out-of-plane alignment will develop in case of TiN zone II depositions [10] and according to Christensen et al. that a (111) out-of-plane alignment will develop in case of YSZ zone II depositions [2]. This because (200) or (111) out-of-plane aligned grains have a lower surface and thus also overall energy than the other out-of-plane oriented grains for TiN and YSZ respectively. This is in accordance with the experimental observations for both materials as summarized in V.4. 
At lower mobility, no re-structurative growth is possible and the several grains and/or columns will compete with each other in an evolutionary way if the mobility is that high that the adparticles may diffuse from one grain to another grain, i.e. zone $\mathrm{T}$ conditions (see chapter II). It was also discussed in chapter II that the grains with the fastest growing direction perpendicular to the substrate will envelop and thus overgrow the other grains resulting in $\mathrm{V}$ shaped columns with faceted column tops. Since the entire film can not aim to its lowest energy because only surface diffusion is allowed, the out-of-plane alignment is kinetically limited. In order to completely understand the resulting out-of-plane orientation, it still should be understood why the [111] direction is the fastest growing direction in case of TiN. And also why the fastest growing direction changes from [100] to [110] when increasing the $\mathrm{O}_{2}$ flow from 3 to $5 \mathrm{sccm}$ in case of YSZ. This will be discussed in the next section.

Finally at still lower mobility, the diffusion of the adparticles is limited to one grain (zone Ic) and no competitive growth happens. This means that no preferential out-of-plane orientation will develop, but that the film consists of columns which remained their initial out-of-plane orientation (see chapter II and table II.5). Again the resulting out-of-plane orientation is a result of a kinetically limited growth. This agrees with the observed random out-of-plane orientation of the TiN film deposited with an $\mathrm{N}_{2}$-flow of $3 \mathrm{sccm}$.

It can be concluded that the transition in out-of-plane orientation from random to a [111] and finally to [200] in case of TiN and from [100] to a mixture of [111] and [110] in case of YSZ can be explained by a transition from a kinetically limited to a thermodynamically determined growth. However, this model should be further developed, in order to understand why a certain direction is the fastest growing direction at the given deposition conditions, what will be done in the next section.

\section{6: Proposed model for the out-of-plane alignment in zone $T$}

The observations described in V.2 and V.3 and the discussion about the different models for the out-of-plane alignment in section V.5 strongly suggest that the influence of an increasing reactive gas flow on the microstructure and out-of-plane alignment can be understood in terms of a transition from kinetically limited to a thermodynamically enhanced growth.

If the deposition is carried out in zone II conditions, re-structurative growth may happen and the resulting film characteristics will be thermodynamically determined. Since the plane of lowest surface energy is known for most materials, prediction of the resulting out-of-plane alignment is evident.

However, predicting the resulting out-of-plane alignment for depositions carried out in zone T conditions is not so straightforward, since it must be known which direction has the largest geometric growth rate at the given deposition conditions. It was already discussed in chapter II that the crystallographic plane that offers the lowest number of nearest neighbours to the incoming adparticles will have the lowest growth rate leading to the specific and unequivocally crystal habit, at least if there is only one crystallographic plane of lowest growth rate. This growth of the grains according to a specific crystal habit causes an anisotropy in geometric growth rate. The grains oriented with the geometric fastest growing direction perpendicular to the substrate will overgrow all other grains. It the next discussions, it will be shown how it can be calculated how many nearest neighbours a plane offers to the adparticles and how it can be calculated whether the grains are clearly faceted or not.

What is the fastest growing direction at given circumstances? In order to find out which crystallographic plane offers the largest number of nearest neighbours, each possible crystallographic plane should be considered. In practice, the 
calculation was limited to the three low index planes: $\{100\},\{110\}$ and $\{111\}$ for a fluorite and rocksalt structure.

Of course that number of offered nearest neighbours to the adparticles not only depends on the kind of incoming adparticles, but also on the termination of the growing plane.

At the used deposition conditions (see V.2 and V.3), it was calculated from the total pressure $P_{\text {tot }}$, the $\mathrm{Ar} / \mathrm{O}_{2}$ or $\mathrm{Ar} / \mathrm{N}_{2}$ flow ratio and the deposition rate $R_{d}$ that the flux of reactive gas towards the substrate is always at least thirty times higher than the flux of deposited metallic particles. Therefore, the state of the reactive gas should be considered to know the termination of a growing plane.

That reactive gas can take part as molecular but also as atomic gas. As seen in chapter I (section I.2), atomic gas can chemisorb on the growing film without having to cross an energy barrier, while molecular gas has to cross the activation barrier $E_{a c t}$ before chemisorbing on the growing plane. Hence, it will be more difficult for the molecular gas to bind on a growing plane. Similar to Shin [39], it was assumed that atomic gas will bind to a growing plane as soon as it finds one nearest neighbour, while molecular reactive gas will only chemisorb and bond to a growing plane if it finds the half of its maximum number of nearest neighbours. It can be concluded that $\mathrm{O}_{2}$ will dissociatively chemisorb on a growing fluorite plane as it finds at least 2 nearest neighbours, while $\mathrm{N}_{2}$ will bind on a rocksalt as it finds at least 3 nearest neighbours.

- $\underline{\text { TiN: }}$

Considering such bulk terminated TiN $\{100\},\{110\}$ and $\{111\}$ planes in case of a molecular reactive gas, the offered number of nearest neighbours to a Ti adatom is 1,2 and 3 respectively, as seen in figure V.6.1.

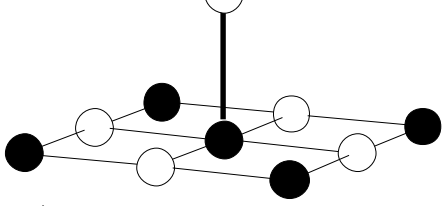

a)

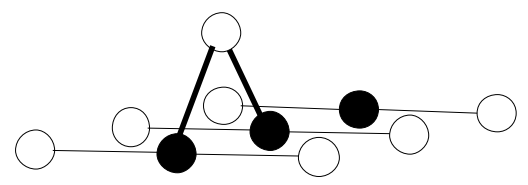

6)

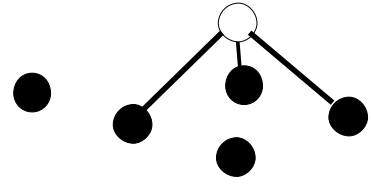

c)

Figure V.6.1: Schematic representation of the number of nearest neighbours offered to a Ti adatom in case of a $\{100\}$ (a), $\{110\}$ (b) and $\{111\}$ (c) bulk terminated TiN plane. The white atoms represent Ti atoms, while the black atoms represent $\mathrm{N}$ atoms.

The number of nearest neighbours that a TiN plane offers, changes when the adparticle is a TiN admolecule instead of a Ti adatom, because such a TiN admolecule can also find Ti atoms as nearest neighbours. In case of a TiN admolecule, the number of nearest neighbours offered by $\{100\},\{110\}$ or $\{111\}$ planes changes to 2,4 and 3 respectively, what is shown in figure V.6.2.

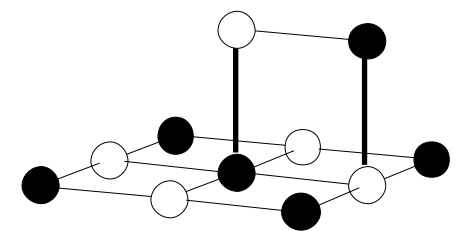

a)

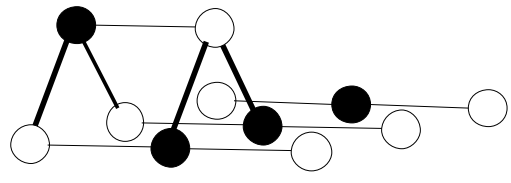

6)

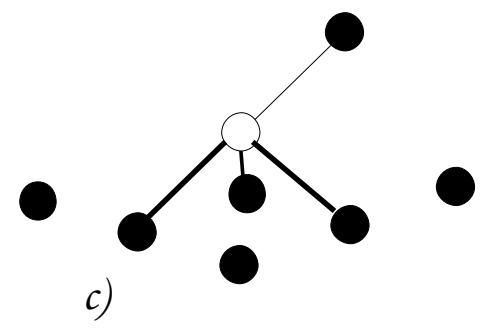

Figure V.6.2: Schematic representation of the number of nearest neighbours offered to a TiN admolecule in case of a $\{100\}$ (a), $\{110\}$ (b) and $\{111\}$ (c) bulk terminated TiN plane. The white atoms represent Ti atoms, while the black atoms represent $\mathrm{N}$ atoms.

As already mentioned, not only the kind of adparticles influences the number of nearest neighbours, but also the termination of the growing plane. In the above examples, bulk 
terminated planes were assumed, which is only valid when the reactive gas acts as molecular gas. At some conditions, the reactive gas rather acts as atomic gas and hence sticks to the growing plane as soon as it finds only one metallic nearest neighbour. Such a transition from molecular to atomic reactive gas alters the termination of the growing plane. Figure V.6.3. shows the number of nearest neighbours for a Ti adatom on a TiN plane with the altered termination. For clarity, this "altered" situation is called the atomic reactive gas situation.

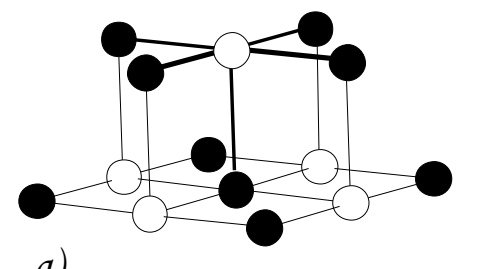

a)

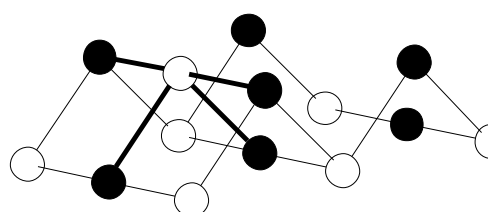

6)

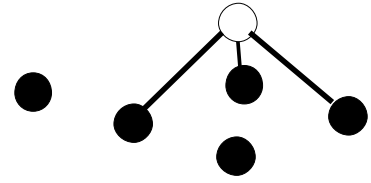

c)

Figure V.6.3: Schematic representation of the number of nearest neighbours offered to a Ti adatom in case of a $\{100\}$ (a), $\{110\}$ (b) and $\{111\}$ (c) atomic reactive gas terminated plane. The white atoms represent Ti atoms, while the black atoms represent $\mathrm{N}$ atoms.

As seen in figure V.6.3, the termination of the $\{100\}$ and $\{110\}$ planes is changed due to the presence of single or double bond $\mathrm{N}$ atoms. The termination of a $\{111\}$ plane didn't change as compared to the molecular gas regime, since it was already fully $\mathrm{N}$-terminated. From figure V.6.3, it is seen that $\{100\},\{110\}$ and $\{111\}$ planes offer 5,4 and 3 nearest neighbours respectively to a Ti adatom, in case of atomic reactive gas. Changing the state of the adparticle from $\mathrm{Ti}$ to $\mathrm{TiN}$ will not change the number of nearest neighbours in the atomic reactive gas situation, since it can be seen in figure V.6.3 that the $\mathrm{N}$-atom of the TiN admolecule will not find any nearest neighbour. Thus, the number of nearest neighbours offered to TiN admolecules are also 5, 4 and 3 for a $\{100\},\{110\}$ and $\{111\}$ plane respectively, in the atomic reactive gas situation.

It can be concluded that the number of nearest neighbours offered to an adparticle depends on the state of the reactive gas (atomic or molecular) and on the state of the adparticle (Ti adatom or TiN admolecule). Of course, more complex adparticles like e.g. $\mathrm{TiN}_{2}$ should be treated. However, it could be shown by mass spectroscopy that $\mathrm{Ti}$ and $\mathrm{TiN}$ are the main adparticles (see figure V.6.4).

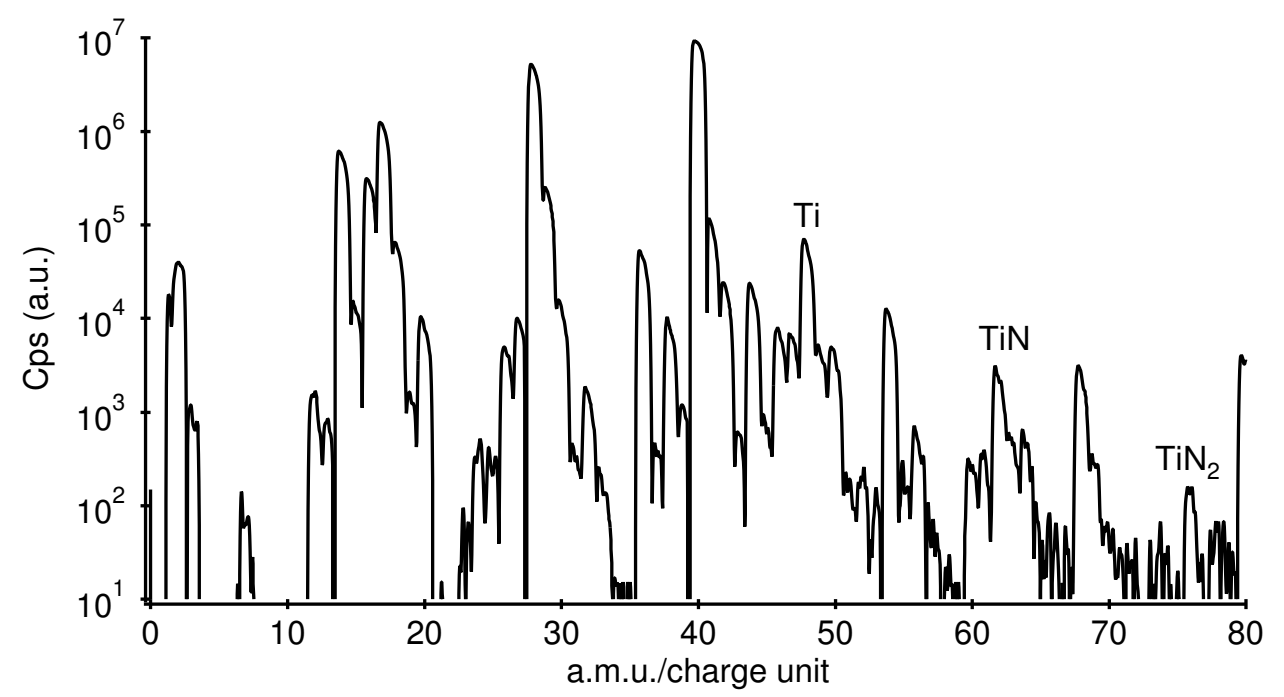

Figure V.6.4: Mass-scan of a plasma during the reactive sputtering of a Ti target in an $\mathrm{Ar} / \mathrm{N}_{2}$ mixture. Complex ions such as $\mathrm{TiN}_{2}$ and should have a mass of 76 a.m.u. As seen in the scan, its amount is negligible compared to the amount of Ti (a.m.u. 48) and TiN (a.m.u. 62). 
- YSZ:

In a complete analogous way, the number of nearest neighbours during the deposition of YSZ could be found. Now, the termination of the growing plane is influenced by the transition from molecular $\mathrm{O}_{2}$ to atomic $\mathrm{O}$, while the state of the adparticles can be $\mathrm{Zr}(\mathrm{Y})$ or $\mathrm{ZrO}$ (YO). Again it could be shown by mass spectroscopy that more complex adparticles like $\mathrm{ZrO}_{2}$ can be neglected (see figure V.6.5).

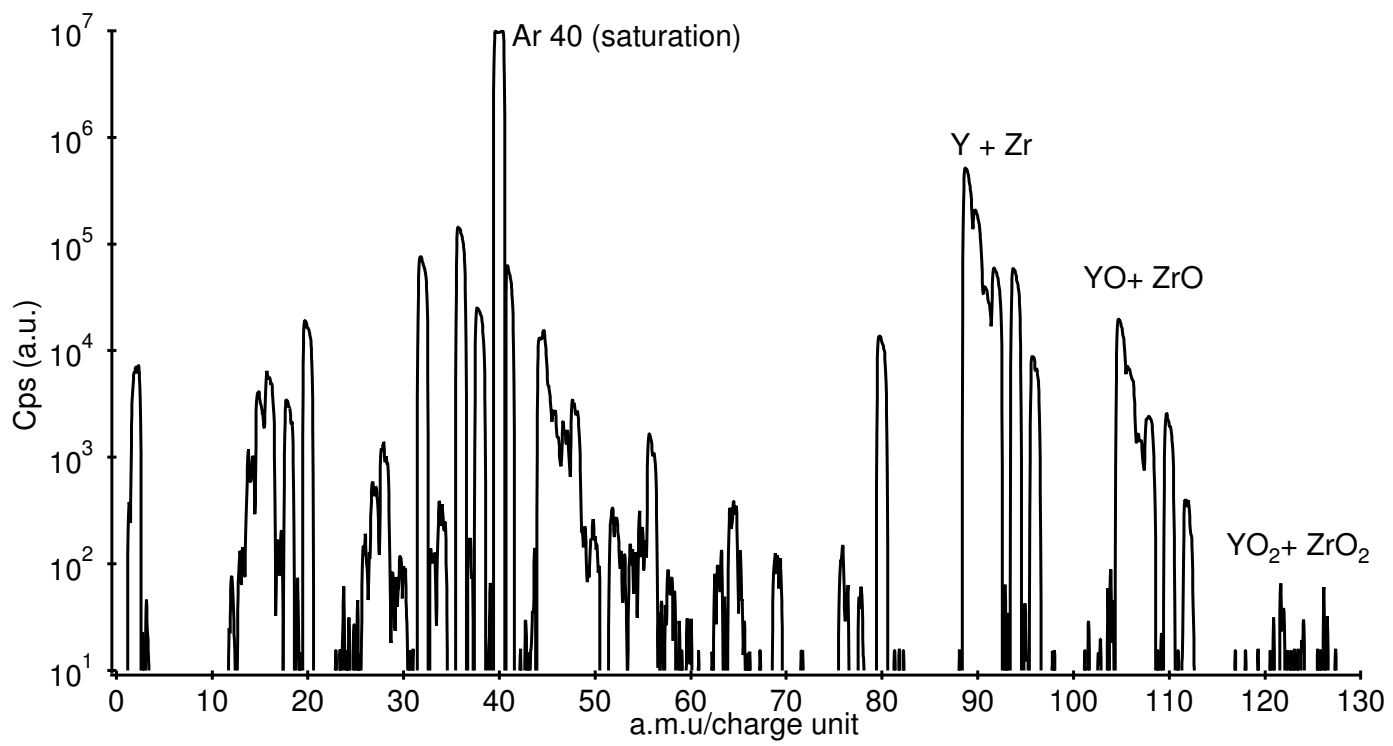

Figure V.6.5: Mass-scan of a plasma during the reactive sputtering of a $\mathrm{Zr} / \mathrm{Y}$ target in an $\mathrm{Ar} / \mathrm{O}_{2}$ mixture. Complex ions such as $\mathrm{YO}_{2}$ and $\mathrm{ZrO}_{2}$ should have a mass of 121 and 122 a.m.u. As seen in the scan, their amount is negligible compared to the amount of Y (a.m.u. 89) and YO (a.m.u. 105).

\section{- TiN and YSZ:}

The number of nearest neighbours during the growth of TiN and YSZ planes in all four possible situations are summarized in table V.6.6. As was reasoned in chapter II, the plane that offers the lowest number of nearest neighbours will have the lowest crystallographic growth rate. For each material and each situation, that lowest number of nearest neighbours is shown in bold and the corresponding direction with lowest growth rate is mentioned in a separate column. It was also discussed in chapter II that the plane with lowest perpendicular growth rate will form the facets of the resulting crystal habit. This resulting crystal habit is also mentioned in a separate column of table V.6.6. It is observed that only two crystal habits, i.e. the $\{100\}$ and the $\{111\}$ develops. Schematic drawings of both crystal habits are shown in figure V.6.7. This growth according to a specific crystal habit causes an anisotropy in geometric growth rate. The direction with the largest geometric growth rate will become the resulting preferential out-of-plane orientation and is also mentioned in the table.

Table V.6.6: The number of nearest neighbours offered by a growing plane for TiN and YSZ in each possible situation. For each situation, the largest number of nearest neighbours is in bold, while the corresponding fastest growing crystallographic plane is mentioned in a separate column. Also the resulting crystal habit is mentioned.

\begin{tabular}{|c|c|c|c|c|c|c|c|}
\hline adparticles & state of the & \multicolumn{3}{|c|}{ number of nearest neighbours: } & slowest cryst. & crystal & fastest geom. \\
\hline & reactive gas & $\{100\}$ & $\{110\}$ & $\{111\}$ & direction & habit & direction \\
\hline $\mathrm{Ti}$ & $\mathrm{N}_{2}$ & 1 & 2 & 3 & {$[100]$} & $\{100\}$ & [111] \\
\hline $\mathrm{Ti}$ & $\mathrm{N}$ & 5 & 4 & 3 & [111] & $\{111\}$ & {$[100]$} \\
\hline $\mathrm{TiN}$ & $\mathrm{N}_{2}$ & 2 & 4 & 3 & {$[100]$} & $\{100\}$ & {$[111]$} \\
\hline $\mathrm{TiN}$ & $\mathrm{N}$ & 5 & 4 & 3 & [111] & $\{111\}$ & [100] \\
\hline $\mathrm{Zr} / \mathrm{Y}$ & $\overline{\mathrm{O}_{2}}$ & 4 & 2 & $\mathbf{1}$ & [111] & $\{111\}$ & [100] \\
\hline $\mathrm{Zr} / \mathrm{Y}$ & $\mathrm{O}$ & 4 & 6 & 4 & $\mathrm{NO}$ & $\mathrm{NO}$ & $\mathrm{NO}$ \\
\hline $\mathrm{ZrO} / \mathrm{YO}$ & $\mathrm{O}_{2}$ & 4 & 3 & 2 & [111] & $\{111\}$ & [100] \\
\hline $\mathrm{ZrO} / \mathrm{YO}$ & $\mathrm{O}$ & 4 & 6 & 4 & $\mathrm{NO}$ & $\mathrm{NO}$ & $\mathrm{NO}$ \\
\hline
\end{tabular}


From table V.6.6 it can be concluded that a material with a rocksalt structure, can have a $\{100\}$ or a $\{111\}$ crystal habit, depending on the situation (adatom/admolecules and atomic/molecular reactive gas). In case of a material with a fluorite structure, the state of the adparticles $(\mathrm{Zr} / \mathrm{Y}$ or $\mathrm{ZrO} / \mathrm{YO})$ does not influence which direction is the slowes growing direction. In case of a molecular reactive gas, the [111] direction is the slowest growing direction, resulting in a $\{111\}$ crystal habit.
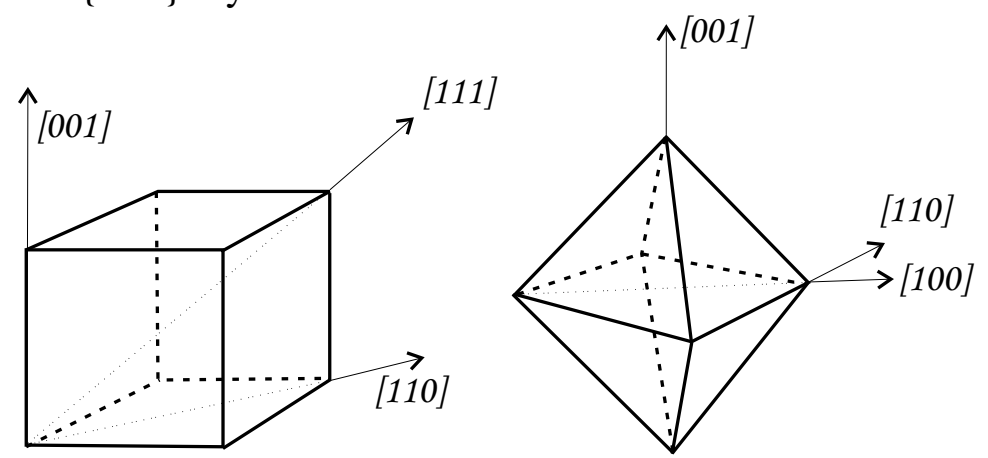

(a)

(b)

Figure V.6.7: A representation of a $\{100\}$ (a) and a $\{111\}$ (b) faceted crystal habit. In case of a $\{100\}$ habit, the [100] plane has the lowest crystallographic growth rate and, while the [111] direction has the largest geometric growth rate. In case of a $\{111\}$ habit, the [111] direction is the slowest crystallographic growing direction, while the [100] direction has the largest geometric growth rate.

It is also observed that in case of an YSZ deposition in the situation of atomic $\mathrm{O}$ gas, no clear and unequivocally crystal habit develops since not one, but two crystallographic planes have the lowest growth rate. This absence of a clear habit destroys the evolution towards a preferential [110] out-of-plane orientation although it has the largest growth rate at the given conditions.

It can be concluded that the resulting preferential out-of-plane orientation and the resulting crystal habit for depositions carried out in zone T conditions is known, as soon as it is known in which situation (adatoms/admolecules and atomic/molecular reactive gas) the deposition is carried out.

How is it known in which situation the deposition is carried out?

This situation depends on the state of the adparticles (admolecules or adatoms) and on the state of the reactive gas (atomic or molecular), which both can be determined by means of energy-resolved mass spectrometry.

Also the ion-to-atom ratio impinging on the growing film can be determined by means of mass spectrometry.

It can be concluded that the resulting microstructure and out-of-plane orientation can be fully understood when carrying out energy resolved mass spectroscopy analysis of the plasma in front of the substrate. This because an energy resolved mass spectrometer provides knowledge about the ion-to-atom ratio -which is related to the energy flux- and about the plasma composition.

The way these measurements are carried out, the results and the discussion of them are described in the following paragraphs.

\section{a: Experimental results}

The energy-resolved mass spectrometer (a Plasma Process Monitor PPM 422 from Pfeiffer Vacuum) allows investigating the neutral particles and the positive ions entering the orifice of the spectrometer. During these plasma measurements, exactly the same deposition conditions were used as in section V.2 and V.3. Only the substrate was removed and replaced by the mass spectrometer. As such, the mass spectrometer was mounted with the orifice facing the 
target in the target-substrate axis at a distance of $13 \mathrm{~cm}$. During all measurements, the orifice was kept at floating potential. The mass spectrometer was differentially pumped to a pressure of $10^{-4} \mathrm{~Pa}$. Due to a bad signal-noise ratio for the measurements in the neutral particle mode, only the positive ions $\left(\mathrm{Ar}^{+}, \mathrm{O}^{+}, \mathrm{O}_{2}^{+}, \mathrm{N}^{+}, \mathrm{N}_{2}{ }^{+}, \mathrm{Ti}^{+}, \mathrm{TiN}^{+}, \mathrm{Zr}^{+}, \mathrm{ZrO}^{+}, \mathrm{Y}^{+}, \mathrm{YO}^{+}\right)$were investigated. Before each measurement, it is important to optimise the signal by measuring the plasma potential $\left(V_{p}\right)$, which corresponds to the maximum of the ion energy distribution of $\mathrm{Ar}^{+}$[40]. Hence, at every deposition condition, an energy-scan at fixed mass of 36 a.m.u. (isotope of Ar) was carried out in order to know the plasma potential. This isotope of Ar was used, because the detector is in saturation when measuring the peak at 40 a.m.u.. Once the plasma potential $V_{p}$ was determined, a mass-scan at fixed energy $\left(\mathrm{E}=V_{p}\right)$ was performed. The measured intensities of the peaks corresponding with the above mentioned positive ions were used for further calculations.

During the plasma measurements, only the positive ions entering the mass-spectrometer were measured, while not all arriving particles at the substrate are positively ionized. As already mentioned in chapter III, the total ionisation in a usual sputter plasma is less than $0.1 \%$. Hence, the measured peak intensities should be recalculated in order to know the amount of neutral particles.

Carrying out a mass-scan and measuring the peak intensities gives a value for the amount of positive ions with a certain mass per unit charge. For all further calculations, it was assumed that the ions are singly positively charged. However, to know the amount of positive ions of a certain element, one should take into account the relative abundance of the used mass-isotope. The used isotopes and their relative abundance are summarized in table V.6.8.

Table V.6.8: All used isotopes and their relative abundance.

\begin{tabular}{|l|l|l|}
\hline El. & a.m.u. & Rel. abund. [\%] \\
\hline $\mathrm{N}_{2}{ }^{+}$ & 28 & 99.269 \\
\hline $\mathrm{O}_{2}{ }^{+}$ & 32 & 99.519 \\
\hline $\mathrm{Ar}^{+}$ & 36 & 0.337 \\
\hline $\mathrm{Ti}^{+}$ & 48 & 73.94 \\
\hline $\mathrm{Y}^{+}$ & 89 & 100 \\
\hline $\mathrm{Zr}^{+}$ & 90 & 51.46 \\
\hline $\mathrm{TiN}^{+}$ & 62 & 73.69 \\
\hline $\mathrm{YO}^{+}$ & 105 & 51.375 \\
\hline $\mathrm{ZrO}^{+}$ & 106 & 99.759 \\
\hline
\end{tabular}

- Ion-to-atom ratio.

First of all, the ion-to-atom ratio was investigated as a function of the reactive gas flow during the growth of TiN and YSZ. Therefore, the amount of positive ions was calculated and afterwards divided by the amount of metallic particles arriving at the substrate (or mass spectrometer).
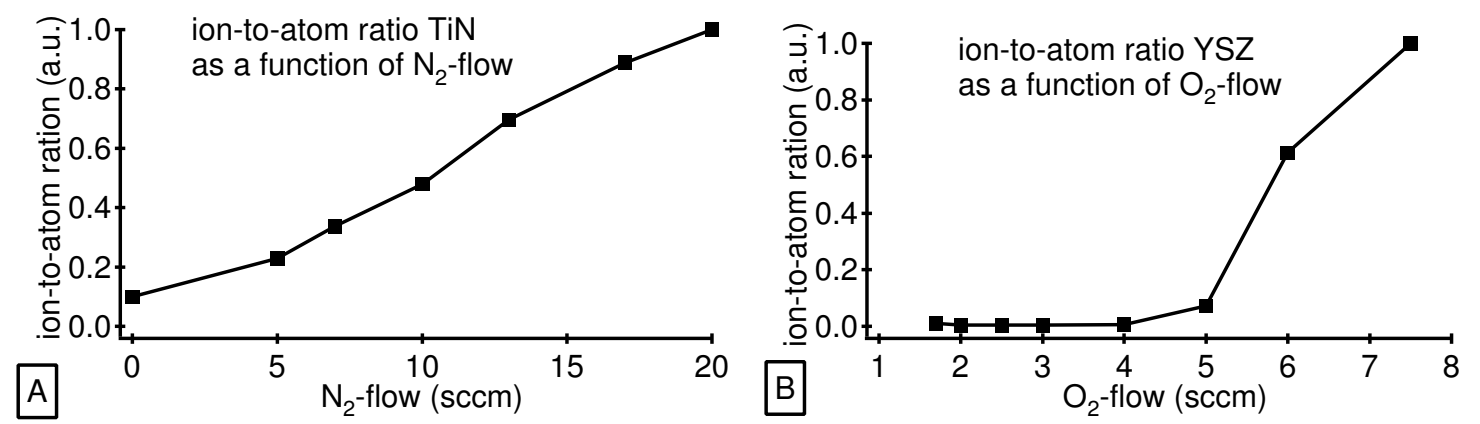

Figure V.6.9: Ion-to-atom ratio in arbitrary units during the deposition of TiN (a) and YSZ (b) as a function of the reactive gas flow. 
Due to the unknown ionisation probabilities of $\mathrm{Ti}, \mathrm{Zr}$ and $\mathrm{Y}$, the absolute values of the ion-toatom ratios $\left(\mathrm{Ar}^{+}+\mathrm{N}_{2}^{+}\right) / \mathrm{Ti}$ and $\left(\mathrm{Ar}^{+}+\mathrm{O}_{2}^{+}\right) /(\mathrm{Zr}+\mathrm{Y})$ could not be calculated. Therefore, the normalized values of $\left(\mathrm{Ar}^{+}+\mathrm{N}_{2}^{+}\right) / \mathrm{Ti}^{+}$or $\left(\mathrm{Ar}^{+}+\mathrm{O}_{2}^{+}\right) /\left(\mathrm{Zr}^{+}+\mathrm{Y}^{+}\right)$are shown in figure V.6.9 and give the ion-to-atom ratio in arbitrary units. Hereby, it is assumed that the ionisation degree of $\mathrm{Ti}$ and $\mathrm{Zr}+\mathrm{Y}$ (and later on also the other elements) remains constant although the partial pressure of the reactive gas was increased. Following the measurements of Konstantinidis et al. [41], this assumption is allowed. Comparing these ion-to-atom ratios as a function of the reactive gas flow, with the energy flux towards the growing film as a function of the reactive gas flow (see fig. V.5.14 and V.5.15), it is clearly seen that both parameters behave similar. Hence, it can be concluded that the influence of the reactive gas flow on the mobility of the adparticles can also be investigated by measuring the ion-to-atom ratio instead of calculating the energy flux.

- State of the adparticles: adatoms or admolecules.

Secondly, it was investigated whether the metallic particles impinge the substrate as an atom ( $\mathrm{Ti}, \mathrm{Zr}$ or $\mathrm{Y}$ ) or as a compound molecule ( $\mathrm{TiN}, \mathrm{ZrO}$ or $\mathrm{YO}$ ). The calculated values of $\left(\mathrm{Ti}^{+} / \mathrm{TiN}^{+}\right)$and $\left(\mathrm{Zr}^{+}+\mathrm{Y}^{+}\right) /\left(\mathrm{ZrO}^{+}+\mathrm{YO}^{+}\right)$are shown in figure $\mathrm{V} \cdot 6.10$. Again, due to the unknown ionisation probabilities of these different particles, the ratios $(\mathrm{Ti} / \mathrm{TiN})$ and $(\mathrm{Zr}+\mathrm{Y}) /(\mathrm{ZrO}+\mathrm{YO})$ can only be given in arbitrary units.
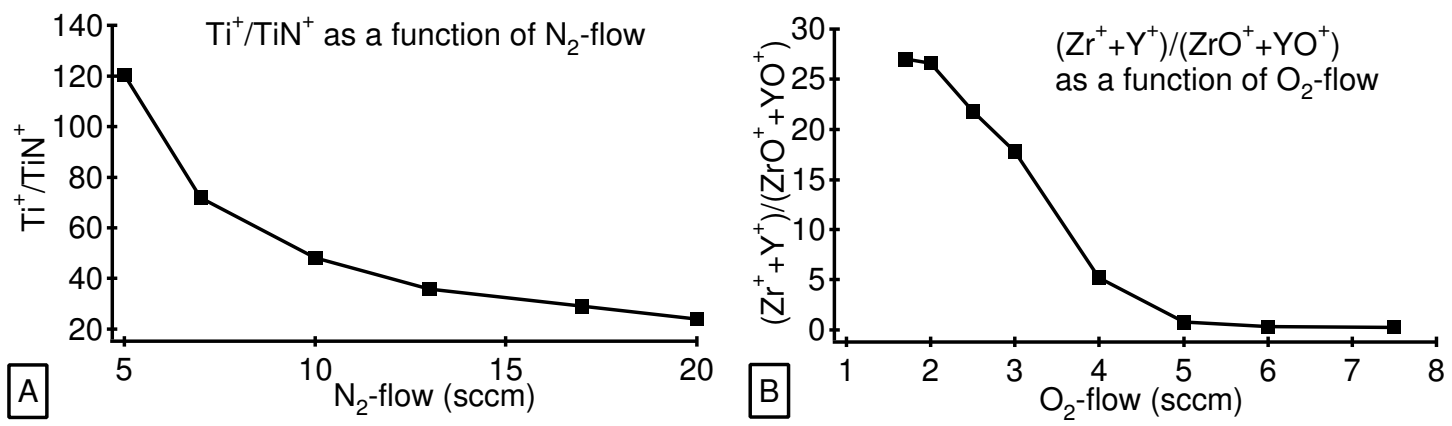

Figure V.6.10: Ratio of $\mathrm{Ti}^{+} / \mathrm{TiN}^{+}(\mathrm{a})$ and $\left(\mathrm{Zr}^{+}+\mathrm{Y}^{+}\right) /\left(\mathrm{ZrO}^{+}+\mathrm{YO}^{+}\right)(\mathrm{b})$ arriving at the substrate during the deposition of TiN and YSZ as a function of the reactive gas flow.

- Termination of the growing plane: atomic or molecular reactive gas.

Finally, mass spectrometry was carried out in order to know the ratio between the flux of metallic single atoms ( $\mathrm{Ti}, \mathrm{Zr}$ or $\mathrm{Y}$ ) impinging the substrate to the flux of atomic reactive gas ( $\mathrm{N}$ or $\mathrm{O}$ ) impinging from the plasma onto the substrate during the growth of TiN and YSZ. The ratios $\mathrm{Ar}^{+} / \mathrm{N}_{2}{ }^{+}$and $\mathrm{Ar}^{+} / \mathrm{O}_{2}{ }^{+}$were calculated. The ionisation probabilities -which are electron energy dependent- of these three elements $\left(\mathrm{Ar}, \mathrm{N}_{2}\right.$ and $\left.\mathrm{O}_{2}\right)$ are known and listed by the National Institute of Standards and Technology [42]. After measuring the electron energy distribution in the plasma by a Langmuir probe, the ratios $\mathrm{Ar} / \mathrm{N}_{2}$ and $\mathrm{Ar} / \mathrm{O}_{2}$ in the plasma can be calculated. Knowing the total pressure $P(0.55 \mathrm{~Pa})$ and the ratios $\mathrm{Ar} / \mathrm{N}_{2}$ and $\mathrm{Ar} / \mathrm{O}_{2}$, it was possible to calculate the partial pressure $P_{r}$ of the reactive gas. Once this reactive gas partial pressure is known, the impinging flux $\Theta_{i}$ of the reactive gas molecules on the substrate can be calculated from the formula $\Theta_{i}=P_{r} /(2 \pi M k T)^{1 / 2}$ as given in chapter I.

Also the ratios $\mathrm{N}^{+} / \mathrm{N}_{2}{ }^{+}$and $\mathrm{O}^{+} / \mathrm{O}_{2}{ }^{+}$were calculated. Again all the ionisation probabilities of these four elements $\left(\mathrm{N}, \mathrm{N}_{2}, \mathrm{O}\right.$ and $\left.\mathrm{O}_{2}\right)$ are listed by the National Institute of Standards and Technology [42]. As such, the ratios $\mathrm{N} / \mathrm{N}_{2}$ and $\mathrm{O} / \mathrm{O}_{2}$ could be calculated. Knowing the dissociation degree of the reactive gas and knowing the impinging rate of the reactive gas molecules, the impinging rate of the atomic reactive gas particles on the substrate could be calculated. 
Also the deposition rate was measured by dividing the thickness of all deposited films by the deposition time. Assuming a sticking coefficient of one for the metallic particles, the impinging rate of the metallic particles on the substrate was calculated. Hence, the absolute values of the ratios $\mathrm{Ti} / \mathrm{N}$ and $(\mathrm{Zr}+\mathrm{Y}) / \mathrm{O}$ could be calculated and are shown in figure V.6.11.
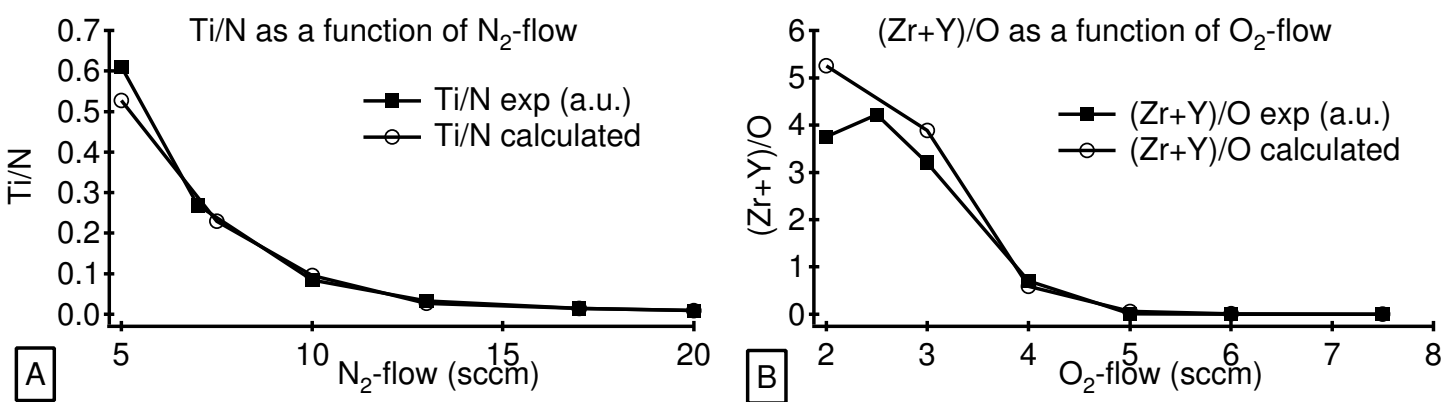

Figure V.6.11: Ratio of Ti/N (a) and $(\mathrm{Zr}+\mathrm{Y}) /(\mathrm{O})$ (b) arriving at the substrate during the deposition of TiN and YSZ as a function of the reactive gas flow.

It can be concluded that by means of mass spectroscopy all needed parameters can be measured, enabling us to understand in which zone (Ic, T or II) and in case of zone T, in which situation (adatoms/admolecules and atomic/molecular reactive gas) the deposition is carried out.

\section{b: Discussion}

- Ion-to-atom ratio.

As already discussed, an increased adparticle mobility during the growth can cause a transition from zone Ic, to zone T and finally zone II. As seen in figure V.6.9.a, the ion-toatom ratio, and hence the adparticle mobility during the TiN deposition, increases with increasing $\mathrm{N}_{2}$-flow. Also during the YSZ depositions, the adparticle mobility increases with increasing $\mathrm{O}_{2}$-flow as shown in figure V.6.9.b. Thus, these observations are in accordance with the suggested transition from zone Ic, to zone T and finally to zone II in case of TiN and the transition from zone $\mathrm{T}$ to a mixture of $\mathrm{T}$ and II in case of YSZ.

However, it should be mentioned that measuring the ion-to-atom ratio is not as instructive as calculating the total energy flux towards the substrate since it doesn't take into account the substrate bias, the energy released during compound formation, energy of the sputtered particles.... Therefore, measuring the ion-to-atom ratio only provides a relative idea of the adparticle mobility, while calculating the energy flux provides a quantitative value.

- State of the adparticles: adatoms or admolecules.

For all depositions carried out in zone $\mathrm{T}$, it should be investigated whether the adparticles are adatoms or admolecules and whether the reactive gas takes part to the growth as atomic or as molecular gas. Therefore, figures V.6.10 and V.6.11 should be analysed.

Let's first look to the state of the adparticles. As seen in figure V.6.10 the ratios $\mathrm{Ti}^{+} / \mathrm{TiN}^{+}$and $\left(\mathrm{Zr}^{+}+\mathrm{Y}^{+}\right) /\left(\mathrm{ZrO}^{+}+\mathrm{YO}^{+}\right)$arriving at the substrate could be measured by mass spectroscopy. As already mentioned, most particles in a plasma remain in the neutral state instead of getting ionized. Therefore, it is straightforward to assume that also a large fraction of the metallic particles will remain in the neutral state, making that not the ratios $\mathrm{Ti}^{+} / \mathrm{TiN}^{+}$and $\left(\mathrm{Zr}^{+}+\mathrm{Y}^{+}\right) /\left(\mathrm{ZrO}^{+}+\mathrm{YO}^{+}\right)$should be known, but the ratios $\mathrm{Ti} / \mathrm{TiN}$ and $(\mathrm{Zr}+\mathrm{Y}) /(\mathrm{ZrO}+\mathrm{YO})$. It would be possible to calculate these ratios when the electron impact ionization cross-sections are known for all these particles. Unfortunately, no values for the ionization cross-sections were found. Therefore, the ratios $\mathrm{Ti} / \mathrm{TiN}$ and $(\mathrm{Zr}+\mathrm{Y}) /(\mathrm{ZrO}+\mathrm{YO})$ can only be given in arbitrary units. 
Nevertheless, a possible transition from metallic adatoms to metallic admolecules can be reasoned in the following way. At zero reactive gas flow, i.e. a deposition in pure Ar, no metallic admolecules such as $\mathrm{TiN}$ or $\mathrm{ZrO}$ and $\mathrm{YO}$ can be formed since there is no reactive gas to react with the target. Here, the interaction between the reactive gas and the target is mentioned, since it can be assumed that nearly no metallic admolecules will form in the gas phase. This because the formation of such a compound needs at least a three-particle interaction, to conserve the momentum and energy. The occurrence of such three-particle collisions in the gas phase can be neglected due to the low pressure $(0.55 \mathrm{~Pa})$.

Hence, at zero reactive gas flow, all metallic adparticles are adatoms. This will remain valid as long as the target is not poisoned. As mentioned in chapter III, a metallic target can get poisoned due to the formation of compound at the target surface. As long as no (or nearly no) compound formation occurs on the target, no metallic admolecules will be sputtered and will thus not reach the growing film. This means that as long as the deposition is carried out in non-poisoned mode, it is reasoned that the adparticles are metallic adatoms and not admolecules. Exceeding the critical reactive gas flow, the target gets poisoned (see chapter III) and a possible transition from adatoms to admolecules can happen.

- In case of the YSZ plasma, an sharp decrease of the ratio $\left(\mathrm{Zr}^{+}+\mathrm{Y}^{+}\right) /\left(\mathrm{ZrO}^{+}+\mathrm{YO}^{+}\right)$is noticed in figure V.6.10b when increasing the $\mathrm{O}_{2}$-flow from 3 to $5 \mathrm{sccm}$. The flow at which this rather sharp change occurs, corresponds with the flow at which the target gets poisoned (see fig.V.4.1), what is in accordance with the above reasoning. This sharp change of the ratio $\left(\mathrm{Zr}^{+}+\mathrm{Y}^{+}\right) /\left(\mathrm{ZrO}^{+}+\mathrm{YO}^{+}\right)$also occurs at the same flow at which a drastic change in microstructure and preferential orientation of the deposited YSZ films was observed (see section V.4). As observed in figure V.6.10b, the ratio $\left(\mathrm{Zr}^{+}+\mathrm{Y}^{+}\right) /\left(\mathrm{ZrO}^{+}+\mathrm{YO}^{+}\right)$drops below one when increasing the $\mathrm{O}_{2}$-flow from 4 to $5 \mathrm{sccm}$, what means that from that moment on more $\left(\mathrm{ZrO}^{+}+\mathrm{YO}^{+}\right)$molecules than $\left(\mathrm{Zr}^{+}+\mathrm{Y}^{+}\right)$atoms arrive at the growing film. Although no values for the ionization cross-sections are known, and thus no definitive conclusion about the $(\mathrm{Zr}+\mathrm{Y}) /(\mathrm{ZrO}+\mathrm{YO})$ ratio can be drawn, a transition from metallic adatoms to metallic admolecules when increasing the reactive gas flow from 3 to $5 \mathrm{sccm}$ seems logic.

- As seen in figure V.6.10a no abrupt change of the ratio Ti/TiN happens. Moreover, it is observed in figure V.6.10a that even at an $\mathrm{N}_{2}$-flow of $20 \mathrm{sccm}$, the flux of $\mathrm{Ti}^{+}$adatoms is still more than 20 times higher than the flux of $\mathrm{TiN}^{+}$admolecules.

Since it seems us illogic that the ionization cross-section of metallic Ti atoms is up to a factor of 20 higher than the ionization cross-section of the TiN molecules, it can be assumed that up to an $\mathrm{N}_{2}$-flow of $20 \mathrm{sccm}$, the metallic adparticles behave essentially as Ti adatoms.

- Termination of the growing plane: atomic or molecular reactive gas.

Secondly, it should also be investigated whether the reactive gas takes part to the growth as atomic or as molecular gas. As previously discussed, the termination of a growing plane changes as soon as an impinging metallic adparticle can not only bind to strongly bonded reactive gas particles but also to weakly bonded reactive gas particles. Here, strongly bond reactive gas means $\mathrm{O}_{2}\left(\right.$ or $\mathrm{N}_{2}$ ) molecules that found more than 1 (or 2) nearest neighbours and thus could undergo dissociative chemisorption. Weakly bond reactive gas means atomic reactive gas which is bond to a growing plane by less than 2 (or 3) nearest neighbours in case of $\mathrm{O}$ (or $\mathrm{N}$ ). It was argued that this weakly bond reactive gas can only originate from atomic reactive gas, since finding 1 (or 2) nearest neighbours is not enough to overcome the energy barrier $E_{a c t}$ and undergo dissociative chemisorption.

Hence, the flux of atomic reactive gas scaled to the flux of impinging metallic particles should be known. It was argued in [43] that this flux of atomic reactive gas can originate from 
reactive gas already dissociated in the gas phase, but also from impinging $\mathrm{O}_{2}$ or $\mathrm{N}_{2}$ which are energetic enough to overcome the energy barrier $E_{\text {act }}$. Mainly, these energetic $\mathrm{O}_{2}$ and $\mathrm{N}_{2}$ particles will be $\mathrm{O}_{2}{ }^{+}$and $\mathrm{N}_{2}{ }^{+}$that are accelerated towards the substrate over the potential difference $\left|V_{p}-V_{s}\right|$.

- $\mathrm{O}_{2}{ }^{+}$and $\mathrm{N}_{2}{ }^{+}$ions will be accelerated towards the substrate since the depositions are carried out on a substrate of which the potential is more negative than the plasma potential $V_{p}$. In case of the TiN depositions, the $\mathrm{N}_{2}{ }^{+}$ions are accelerated towards the substrate since the plasma potential $V_{p}$ is more positive than the grounded substrate potential (see figure V.5.2), while in case of YSZ, the substrate is at floating potential, i.e. 15 volt more negative than the plasma potential (see figure V.5.3).

This flux of atomic reactive gas originating from accelerated $\mathrm{O}_{2}{ }^{+}$and $\mathrm{N}_{2}{ }^{+}$towards the substrate could be calculated from the mass spectrometry results and cylindrical probe measurements. The cylindrical probe was build to the example of Thornton [44] and is a stainless steel cylinder with a diameter of $1 \mathrm{~cm}$ and height of $1 \mathrm{~mm}$, on which a negative bias was applied. Increasing the negative bias, the saturation current of positive ions towards the substrate could be measured. In that way, a quantitative value for the ion-toatom ratio could be measured by means of this cylindrical probe. From the mass spectrometry results, the fraction $\mathrm{O}_{2}^{+} / \sum A^{+}$or $N_{2}^{+} / \sum A^{+}$could be calculated, in which $A$ represent all measured positive ions in the plasma. Taking into account this fraction of $\mathrm{O}_{2}{ }^{+}$ and $\mathrm{N}_{2}{ }^{+}$ions, the $\mathrm{O}_{2}{ }^{+}$-to- $(\mathrm{Zr}+\mathrm{Y})$ and $\mathrm{N}_{2}{ }^{+}$-to-Ti ratio towards the substrate could be calculated. Multiplying this values with two (each $\mathrm{O}_{2}{ }^{+}$and $\mathrm{N}_{2}{ }^{+}$splits up in two atoms), the contribution of energetic ions to the atomic flux of reactive gas is obtained.

- The contribution of the atomic reactive gas originating from the gas phase could be calculated from the energy resolved mass spectrometry results as discussed previously. The ratios $\mathrm{Ti} / \mathrm{N}$ and $(\mathrm{Zr}+\mathrm{Y}) / \mathrm{O}$ are shown in figure V.6.11.

The total flux of reactive gas scaled to the flux of deposited particles as a function of the reactive gas flow is shown in figure V.6.12.

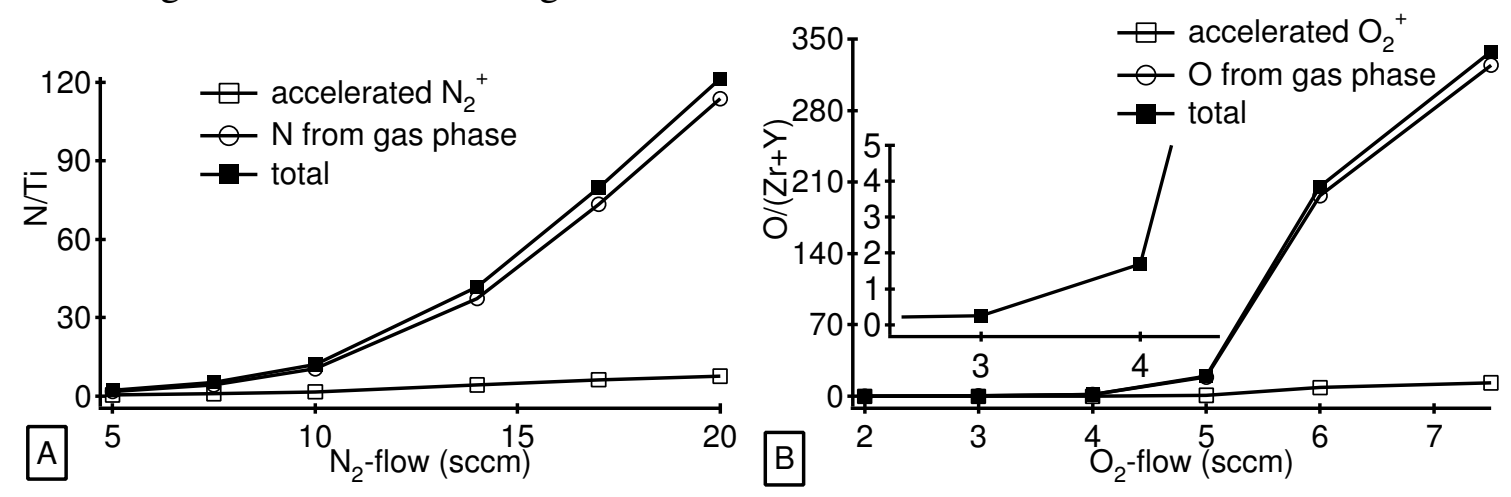

Figure V.6.12: Ratio of N/Ti (a) and $\mathrm{O} /(\mathrm{Zr}+\mathrm{Y})$ (b) arriving at the substrate during the deposition of TiN and YSZ as a function of the reactive gas flow. Also the separate contribution of the two sources (from the gas phase and from accelerated ions) is shown. In case of YSZ, a higher magnification is shown in the inset.

As observed in figure V.6.12, for TiN as well as for YSZ, the flux of atomic reactive gas towards the growing film scaled to the metallic flux increases with increasing reactive gas flow. 
- In case of YSZ (see fig. V.6.12b), it is observed that the flux of atomic reactive gas exceeds the flux of metallic particles (ratio >1) as soon as the reactive gas flow is more than $3 \mathrm{sccm}$ $\mathrm{O}_{2}$.

It can be concluded that in case of the YSZ depositions, increasing the $\mathrm{O}_{2}-$ flow above 3 sccm results in a transition from adatoms to admolecules (see earlier) and also in a transition from the molecular $\mathrm{O}_{2}$ to the atomic $\mathrm{O}$ reactive gas situation at the same critical gas flow.

- However, in case of TiN, the ratio N/Ti is always larger than 1, even at an $\mathrm{N}_{2}$-flow of 5 sccm. Hence, assuming that all impinging atomic $\mathrm{N}$ particles get bond to the growing film, all depositions would be carried out in the atomic reactive gas situation. As seen in table V.6.6, this makes that one should always observe a [100] out-of-plane orientation for depositions carried out in zone $\mathrm{T}$. This does not correspond with the experimental results, since a zone $\mathrm{T}$ with [111] out-of-plane orientation was observed for depositions at low $\mathrm{N}_{2}$-flow ( $\left.\leq 14 \mathrm{sccm}\right)$. Also the results of Greene [16] and Hultmann [17] are seemingly in contradiction with the above reported growth mechanism. They observed a transition from [111] to [100] out-plane oriented TiN films when increasing the ion-to-atom ratio. Since their depositions were carried out in pure $\mathrm{N}_{2}$ at a pressure of $2.7 \mathrm{~Pa}$ and at rather high ion-to-atom ratio, the flux of atomic $\mathrm{N}$ towards the growing film is expected to be high. No details about their plasma composition were reported, but assuming a dissociation degree of about $0.5 \%$ in the plasma and knowing the ion-to-atom ratio, it was calculated that they observed a [111] out-of-plane oriented TiN film even in the presence of a flux of atomic $\mathrm{N}$ which is 60 times higher than the Ti adatom flux. Hence, again, at that high N-flux they should have observed a [100] out-of-plane orientation instead of a [111] out-of-plane orientation at low ion-to-atom ratios.

Fortunately, this seemingly contradiction can be solved when looking to the ab initio calculations, carried out by Gall et al. [45]. He calculated that weakly bond $\mathrm{N}$ atoms, i.e. $\mathrm{N}$ atoms which found only one or two nearest neighbours, are not stable on the surface but are thermodynamically driven to desorb again from the surface and recombine to $\mathrm{N}_{2}$ in the gas phase. Therefore, an atomic $\mathrm{N}$ arriving at the growing film will not chemisorb to that surface, but only adsorb, diffuse some time around and then desorb again. It was calculated that such an $\mathrm{N}$ atom will only chemisorb on the growing surface when it encounters a Ti adatom during its diffusion process. Hence, the TiN depositions can only be carried out in the atomic reactive gas situation if the atomic $\mathrm{N}$ flux is so high that the steady state coverage of diffusing $\mathrm{N}$ adatoms is high enough to bond all Ti adatoms. Gall calculated that the growth rate of [111] and [100] out-plane oriented grains is comparable, and thus that we are in the transition region from molecular to atomic reactive gas, when the ratio of impinging atomic $\mathrm{N}$ to $\mathrm{Ti}$ adatoms is 20. Although this value is somewhat too low to fully explain the experimental observations of our group and these of Greene and Hultman, it gives a solution for the seemingly contradiction. According to our results and also these of Greene and Hultmann, an atomic $\mathrm{N}$ flux of at least 70 times higher than the flux of Ti adatoms is needed to observe a transition from the molecular to the atomic reactive gas situation.

As far as we know, no calculations about the stability of weakly bond $\mathrm{O}$ on an YSZ plane has been carried out. But since $\mathrm{Zr}$ and $\mathrm{Y}$ have both a high affinity for oxygen, it can be assumed that weakly bond $\mathrm{O}$ is stable on an YSZ plane and will not desorb again.

Taking into account that the flux of atomic $N$ should be 70 times higher than the flux of metallic adparticles in order to reach the atomic reactive gas situation, it can be concluded that all TiN depositions of the zone T conditions, i.e. for an $N_{2}$-flow lower than 15 à 16 sccm, are carried out in the situation of metallic Ti adatoms and molecular $\mathrm{N}_{2}$ gas. 
Is this evolutionary overgrowth or 'survival of the fastest' model in accordance with the reported results?

It will be shown that the above suggested mechanism is not only able to explain our own results as described in V.2 and V.3, but also the results of other authors reported in literature.

Let's first discuss the TiN results and then the YSZ results.

$\underline{\mathrm{TiN}}$ :

- As described in V.2 and as summarized in V.4, an increasing $\mathrm{N}_{2}$-flow results in:

- a transition from zone Ic to zone $\mathrm{T}$ and finally into a zone II structure

- a corresponding transition from random out-of-plane oriented to a [111] and finally to a [200] out-of-plane alignment

- a continuous increase in adparticle mobility during the growth.

The energy resolved mass spectrometry showed that:

- all TiN depositions of the zone Ic and zone T conditions, i.e. at an $\mathrm{N}_{2}$-flow up to 15 à $16 \mathrm{sccm}$, are carried out in the situation of metallic Ti adatoms and molecular $\mathrm{N}_{2}$ gas.

As discussed in chapter II and as shown in table II.5, the transition from zone Ic, to zone T and finally zone II is fully in agreement with the observed increase in adparticle mobility.

- The correlation between a zone Ic structure and a random out-of-plane orientation has been discussed in chapter II and is shown in figure II.2.12. Due to the absence of any interaction between the different grains, the random out-of-plane orientation of the initial nuclei is conserved and straight, well faceted but random out-of-plane oriented columns develop.

- Also the correlation between a zone II structure and a [200] out-of-plane alignment is already clear due to the aim for the lowest surface energy.

- Finally, the appearance of a [111] preferential orientation in the depositions with a zone T structure can also be understood since mass spectrometry revealed that the depositions were carried out in such conditions that all adparticles are Ti adatoms and that the reactive gas acts as molecular $\mathrm{N}_{2}$ during the growth. It was previously calculated that in such conditions the [111] direction is the geometric fastest growing direction and [100] the slowest crystallographic growing direction (see table V.6.6). As such, according to the above described model, a [111] out-of-plane alignment will develop in an evolutionary way with $\{100\}$ faceted grains or column tops. This $\{100\}$ faceting was clearly observed by plan view SEM images (e.g. figure V.2.7).

- In literature, Oh and Je [11,12] observed a transition from a preferential [100] to a preferential [111] out-of-plane orientation with increasing thickness. By means of in-situ XRD, TEM and corresponding SAED patterns, they observed that depending on the deposition conditions, this transition occurred at a film thickness of about $150 \mathrm{~nm}$ and that no re-structurative grain growth happened.

Their observations can be understood by means of the above discussed overgrowth model, assuming again that the adparticles are Ti adatoms and that the reactive gas is molecular $\mathrm{N}_{2}$. Unfortunately, no data about their plasma composition has been published.

As calculated previously, under these conditions the [111] direction will have the largest geometric growth rate, while the [100] direction the lowest crystallographic growth rate. This [100] direction has the lowest crystallographic perpendicular growth rate, because the $\{100\}$ plane offers the lowest number of nearest neighbours to the Ti adatoms. According to Huang et al. [46] and as discussed in chapter II, this means that the Ti adatoms have the largest mobility on the $\{100\}$ planes. As shown in figure II.2.6, this means also that the [100] out-ofplane oriented grains will have the largest lateral growth rate. Hence, due to the anisotropy in 
lateral growth rate, a random out-of-plane nucleated film will evolve as shown in figure V.6.13.

Initially the [100] out-of-plane oriented grains have a larger diameter than all other grains, since they offered the largest lateral growth rate. Proceeding the thin film deposition, the [111] out-of-plane oriented grains will slowly envelop the other grains, because they have the largest geometric growth rate. It can be concluded that initially the volume fraction of [100] out-of-plane oriented grains is the largest, while the volume fraction of the [111] out-of-plane oriented grains becomes dominant as soon as they have overgrown the other grains. This exactly corresponds with the observations of Oh and Je, since an XRD $\theta / 2 \theta$ spectrum represents the volume fraction of grains with a specific out-of-plane orientation. The transition height of about $150 \mathrm{~nm}$ at which the out-of-plane orientation changed from [100] to [111] as observed by $\mathrm{Oh}$ and Je, corresponds very well with the observed film thickness in their depositions at which the microstructural overgrowth was completed [11].
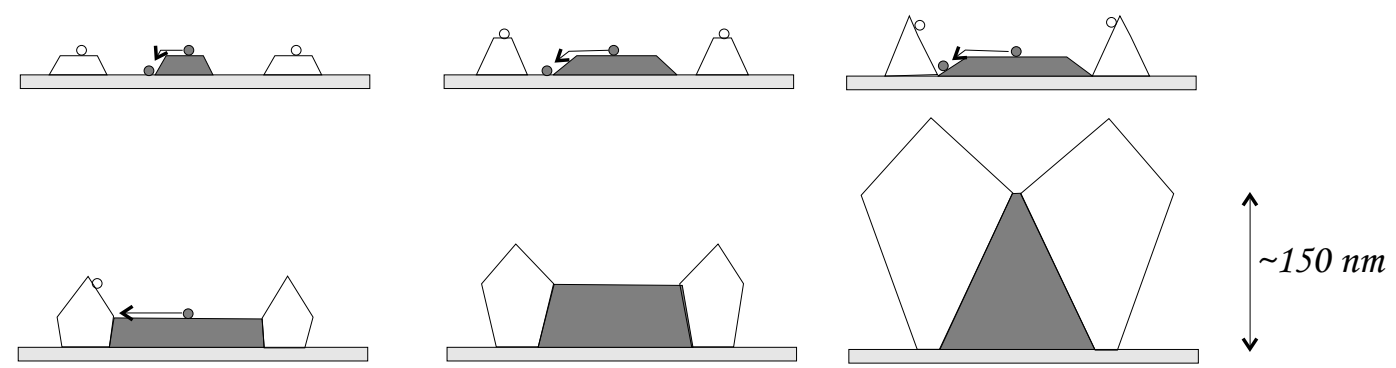

Figure V.6.13: Evolution of the initial growth of a random out-of-plane nucleated film in zone T conditions with the situation of $\mathrm{Ti}$ adatoms and $\mathrm{N}_{2}$ molecular gas. The dark grains are [100] out-of-plane oriented while the [111] out-of-plane oriented grains are white.

- Also the observations of Shin [39] can be explained by the described overgrowth mechanism. Shin deposited TaN films by sputtering a Ta target in a mixture of Ar and $15 \%$ $\mathrm{N}_{2}$, with a total pressure of $2.7 \mathrm{~Pa}$. Similar to TiN, TaN also has a rocksalt structure. By increasing the ion-to-atom ratio towards the growing film, Shin observed a transition from [111] to [100] out-of-plane oriented grains at an ion-to-atom ratio of 6.5. By TEM measurements it was observed that all films show a zone $\mathrm{T}$ structure.

Unfortunately, again no data about their plasma composition is known, but the transition from [111] to [100] zone T out-of-plane aligned films can be understood assuming a transition from Ta adatoms and $\mathrm{N}_{2}$ molecular gas, towards a situation of atomic $\mathrm{N}$ reactive gas. As previously discussed, the [111] direction has the largest geometric growth rate in the situation of Ta adatoms and molecular $\mathrm{N}_{2}$ gas, what explains the [111] out-of-plane alignment. A transition to atomic $\mathrm{N}$ gas causes the [100] direction to be the fastest geometric growing direction, and the [111] the slowest crystallographic growing direction. This explains the [100] out-of-plane alignment with the $\{111\}$ faceted column tops. This transition to atomic $\mathrm{N}$ can be caused by the high $\mathrm{N}_{2}$ partial pressure and the increasing flux of $\mathrm{N}_{2}{ }^{+}$ions towards the substrate, i.e. an increased contribution of dissociative chemisorption.

- Adibi [47], deposited $\mathrm{Ti}_{0.5} \mathrm{Al}_{0.5} \mathrm{~N}$ films by sputtering a Ti/Al target in pure $\mathrm{N}_{2}$ at a pressure of $2.7 \mathrm{~Pa}$ and an ion-to-atom ratio of 12 . Again, the $\mathrm{Ti}_{0.5} \mathrm{Al}_{0.5} \mathrm{~N}$ has a rocksalt structure. He observed a clear [100] out-of-plane alignment with a zone T structure. They also observed by TEM plan view and cross-sections that the column tops were $\{111\}$ faceted. These observations can be understood, assuming an atomic $\mathrm{N}$ gas situation since then these $\{111\}$ planes have the lowest crystallographic growth rate (see table V.6.6). As was discussed in the previous paragraph, atomic reactive gas can originate from dissociative chemisorption of energetic $\mathrm{N}_{2}^{+}$ions and from atomic $\mathrm{N}$ dissociated in the gas phase. Since Adibi uses a high 
ion-to-atom ratio and a high $\mathrm{N}_{2}$ partial pressure, assuming a high flux of atomic $\mathrm{N}$ towards the growing film is not illogic.

- Greene [16] and Hultman [17] also observed a transition from [111] to [100] out-of-plane aligned films when increasing the ion-to-atom ratio. A fully [100] out-of-plane alignment was obtained as soon as the ion-to-atom ratio reached a value of 3.5. Their results should be understood in terms of a transition from kinetically limited to thermodynamically enhanced growth since they observed a corresponding transition from zone T towards zone II.

Since most deposition conditions, the ion-to-atom ratio and also the deposition rate was mentioned in their publications, the energy flux towards the growing film could be estimated. Their depositions were done on an electrically floating substrate. Hence, nearly all electrons were repelled from the growing film, while the positive ions were accelerated by the potential difference $\left|V_{p}-V_{f l}\right|$. This potential difference was about $20 \mathrm{eV}$. The energy released during the formation of a TiN compound is $8.4 \mathrm{eV}$ (see section V.5). The kinetic energy delivered from the sputtered $\mathrm{Ti}$ atoms can be ignored since most sputtered particles will be thermalised before reaching the substrate (total pressure $=2.7 \mathrm{~Pa}$ and target-substrate distance of $6.5 \mathrm{~cm}$ ). Calculating the energy flux towards the substrate for the deposition at an ion-to-atom ratio of 3.5 , an energy flux of $(8.4+20 \times 3.5)=78.4 \mathrm{eV}$ per deposited $\mathrm{Ti}$ atom is obtained. As shown in figure V.5.14, this value corresponds very well with the needed energy per deposited $\mathrm{Ti}$ particle to obtain a zone II growth and thus a [100] out-of-plane alignment in our depositions. The [111] out-of-plane orientation at low ion-to-atom ratios was already discussed in the previous section, and was understood as a zone $\mathrm{T}$ growth in the situation of metallic $\mathrm{Ti}$ adatoms and molecular $\mathrm{N}_{2}$-gas.

$\underline{\text { YSZ: }}$

- Also the reported observations of the YSZ depositions in section V.3 can be understood with the above described model.

A transition from a clear [100] out-of-plane alignment to a mixture of 75\%[111] and 25\% [220] out-of-plane orientation was observed. At the transition region, a considerable amount of [311] out-of-plane oriented grains was observed (see fig. V.3.5). The microstructure changed from zone $\mathrm{T}$ to an unclear structure.

- At low oxygen flow, the [100] out-of-plane orientation can be understood since mass spectrometry indicated that the adparticles are $\mathrm{Zr}$ and $\mathrm{Y}$ adatoms and that the reactive gas acts as molecular $\mathrm{O}_{2}$ gas. According to table V.6.6, the [100] direction has the largest geometric growth rate, while the [111] direction is the slowest crystallographic growing direction. This means that $\{111\}$ faceted, [100] out-of-plane oriented columns will develop, what is in accordance with the observations (see e.g. fig. V.3.6). Also the observation that the [100] out-of-plane oriented grains initially have the smallest diameter (see fig. V.3.7) is in accordance with the proposed model. This because the "high mobility" planes will have initially the largest lateral growth rate, while the [100] out-of-plane oriented grains have the lowest lateral growth rate, since they are "low mobility" grains.

- At higher oxygen flow a mixture of [111] and [220] out-of-plane oriented grains develops. As observed in table V.6.6, the [220] direction has the largest crystallographic growth rate in case that the reactive gas acts as atomic $\mathrm{O}$ gas. As already discussed, mass spectroscopy indeed showed a transition from the molecular to the atomic reactive gas situation when increasing the reactive gas flow from 3 to $5 \mathrm{sccm}$. Unfortunately, these [220] out-of-plane oriented grains don't have a unequivocally determined crystal habit. According to the "survival of the fastest" model as described in chapter II, these grains are not able to overgrow the other out-of-plane oriented grains. Hence, although the [220] direction is the fastest growing direction, not a perfect [220] out-of-plane alignment nor a zone T structure will develop. This impossibility of overgrowth results in the appearance of a second 
mechanism causing another out-of-plane orientation. As shown in figure V.5.15, the strongly increased mobility will enhance the thermodynamic aim for the lowest surface energy. As already discussed, this aim for the lowest surface energy explains the considerable fraction of [111] out-of-plane oriented grains.

Due to the presence of two competing mechanisms, i.e. aim for lowest surface energy and the fastest growth of [220] out-of-plane oriented grains, an unclear microstructure will develop.

This unclear microstructure will be characterised by a continuous re-nucleation and poor or non-uniform faceted grains. Such a structure is observed in the TEM cross-section of an YSZ film deposited at $6 \mathrm{sccm} \mathrm{O}_{2}$ (see fig. V.3.9).

- Also the considerable fraction of [311] out-of-plane oriented grains in the region of 3 à 4 sccm $\mathrm{O}_{2}$, can be understood with the proposed growth mechanism. As shown by the "survival of the fastest" described in chapter II, the grains with the most tilted facets will overgrow the other planes. In case of an YSZ deposition with the situation of molecular $\mathrm{O}_{2}$ gas, this corresponds to [100] out-of-plane oriented grains, faceted by $\{111\}$ planes. Since this overgrowth is purely based on geometric reasons, it is obvious that grains only slightly deviating from this preferred orientation display a slower overgrowth than completely wrong oriented grains. Since the angle between a [100] and a [311] direction is only $25^{\circ}$, it is clear that the [311] out-of-plane oriented grains are overgrown very slowly, what causes a considerable [311] peak in the $\theta / 2 \theta$ spectra. It has been calculated in a similar geometric reasoning by Wada et al. that the [311] out-of-plane oriented grains are overgrown 4 times slower than the [111] out-of-plane oriented grains [48].

It can be concluded that the resulting out-of-plane orientation and microstructure of TiN and YSZ films deposited by unbalanced magnetron sputtering is a result of a competition between kinetically limited and thermodynamically enhanced growth. In case of a kinetically limited growth in zone T conditions, also the plasma composition (adatoms/admolecules and atomic/molecular reactive gas) should be taken into account in order to fully understand the resulting preferential orientation.

The influence of an increasing reactive gas flow on the microstructure and out-of-plane orientation of our TiN and YSZ films has been schematically summarized in figure V.6.14.
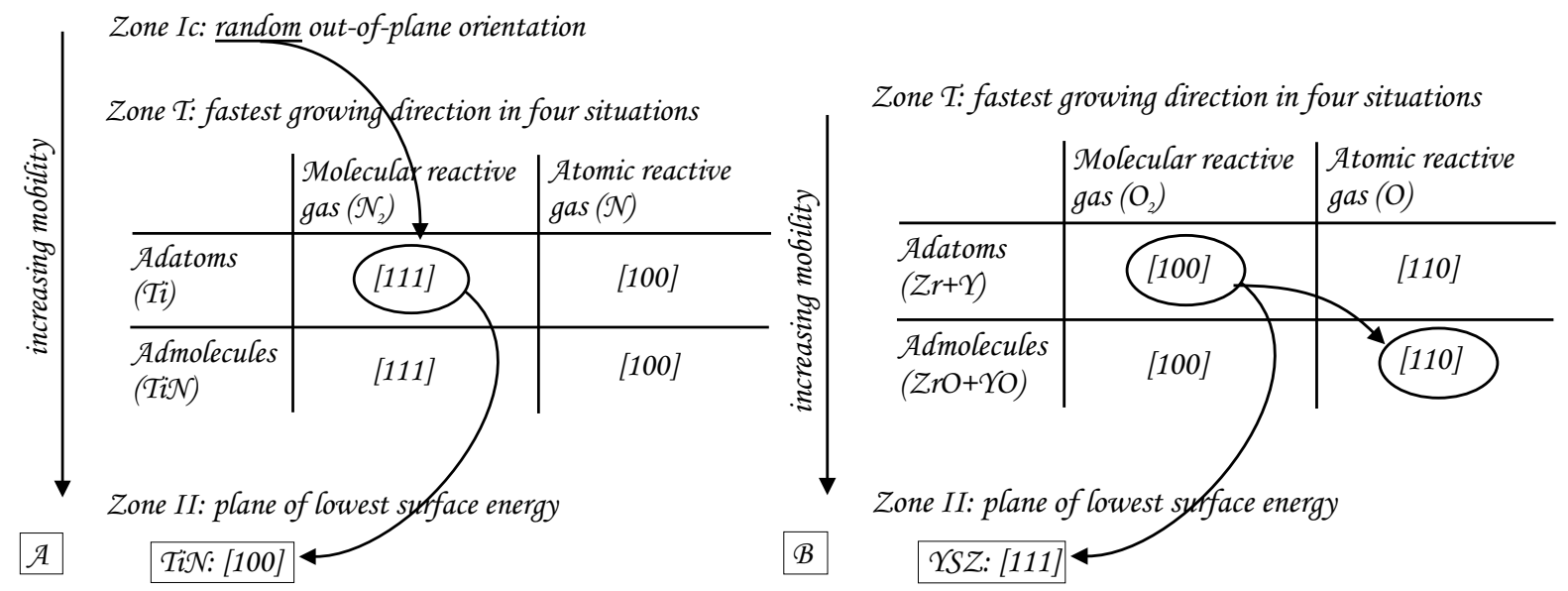

Figure V.6.14: Schematic representation of the influence of an increasing reactive gas flow on the microstructure and out-of-plane orientation in case of TiN (a) and YSZ (b). 


\section{7: Additional experimental observations}

Beside the influence of the reactive gas flow, also the influence of the target-substrate angle, target-substrate distance and substrate bias on the resulting out-of-plane orientation has been investigated.

According to the above described model for the resulting out-of-plane orientation, mainly two parameters influence the out-of-plane orientation: the adparticle mobility and the plasma composition. The above mentioned parameters, i.e. target-substrate angle, target-substrate distance and substrate bias have no or only a minor influence on the plasma composition. Hence, a possible influence of these parameters on the out-of-plane orientation should mainly be understood as a variation of the adparticle mobility during the growth.

Influence of the target-substrate angle $\alpha$ :

The influence of the target-substrate angle $\alpha$ on the resulting preferential orientation has been investigated by depositing YSZ films on a polycrystalline stainless steel substrate at a pressure of $0.55 \mathrm{~Pa}$, a target-substrate distance of $13 \mathrm{~cm}$ and an $\mathrm{O}_{2}$-flow of $2 \mathrm{sccm}$. However, compared to the depositions described in section V.3, the depositions were carried out with a balanced instead of an unbalanced magnetron. As was already mentioned in chapter III, the use of an unbalanced magnetron causes the presence of a plasma plume in front of the substrate and a high energy flux towards the growing film due to the plasma-surface interaction. In case of a balanced magnetron, this plasma plume is not created causing a much weaker plasma-surface interaction during the thin film growth. Hence, the overall adparticle mobility will be lower for depositions with a balanced magnetron compared to similar depositions carried out with an unbalanced magnetron.

The deposited YSZ films were characterised by XRD $\theta / 2 \theta$ and the fraction of grains with a specific out-of-plane orientation was again calculated taking into account the intensity factors (see table V.3.4). The results are shown in figure V.7.1.

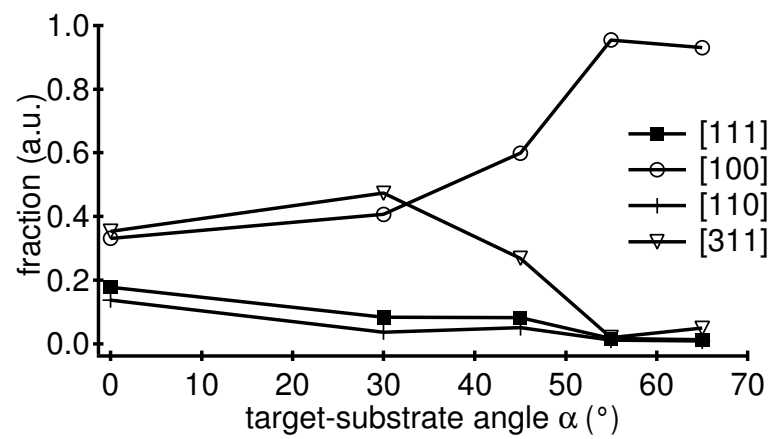

Figure V.7.1: Fraction of grains with a specific out-of-plane orientation as a function of the target-substrate angle $\alpha$, for YSZ films deposited by balanced magnetron sputtering.

It is seen in figure V.7.1 that the films deposited at low target substrate angle have a nearly random out-of-plane orientation. Increasing the target-substrate angle causes the transition to a nearly perfect [100] out-of-plane aligned film. It will be shown that this transition in out-ofplane orientation is caused by a transition from zone Ic to zone T growth.

In section V.3, it was discussed that no zone Ic YSZ films could be deposited with an unbalanced magnetron, since an oxygen flow lower than $2 \mathrm{sccm}$ should be used in order to obtain a lower adparticle mobility. However, an oxygen flow lower than $2 \mathrm{sccm}$ resulted in bad oxidized YSZ films.

Instead of decreasing the adparticle mobility by lowering the $\mathrm{O}_{2}$-flow, it can also be decreased by using a balanced instead of an unbalanced magnetron. Hence, the observation of random out-of-plane oriented films in figure V.7.1 corresponds with the development of a zone Ic structure. The transition to a [100] out-of-plane alignment corresponds with a transition to a 
zone $\mathrm{T}$ deposition, since increasing the target-substrate angle $\alpha$ increases the adparticle mobility in two ways.

First, the overall mobility is increased since the deposition rate decreases with increasing tilt angle. Decreasing the deposition rate and maintaining the energy flux due to electron and ion bombardment, causes an increase in the delivered energy per deposited particle.

Second, the probability that an adparticle diffuses from one grain to another and thus promotes the competition between different grains increases because the net diffusion travel distance of an adparticle increases with increasing target-substrate angle. This net diffusion travel distance increases because the diffusion gets more directed instead of random due to the conservation of the momentum of the adparticles as suggested by Abelmann [49] and as simulated by Kools [50].

Finally, increasing the target-substrate angle not only promotes the zone $\mathrm{T}$ growth with respect to a zone Ic growth due to an increased adparticle mobility, but also fastens the overgrowth process. Increasing the target-substrate angle increases the shadowing effect as calculated by Dong et al. [51]. This means that the higher grains will catch a higher flux of adparticles and will also "shadow" the lower grains behind them, as also shown in figure II.2.1. This shadowing effect will promote the growth of the higher grains with respect to the lower grains, what can only accelerate the overgrowth process in zone $\mathrm{T}$ conditions. This shadowing effect will also offer the probability that the columns are slightly tilted towards the material flux, in case of deposition on a tilted substrate.

It can be concluded that the influence of the target-substrate angle on the resulting out-ofplane orientation can be fully understood with the growth model proposed in section V.6. It was shown that increasing the target-substrate angle promotes a zone $\mathrm{T}$ growth with respect to a zone Ic growth. On the other hand, the transition between a zone T and zone II growth is not strongly influenced by the target-substrate angle. The overall adparticle mobility increases with increasing target-substrate angle, favouring the zone II growth. While increasing the shadowing effect will harm the re-structurative grain growth, favouring the zone T growth. Hence, both processes counteract each other and no real influence of the target-substrate angle on the transition from zone T to zone II will be noticed.

\section{Influence of the target-substrate distance:}

The depositions described in section V.2, i.e. TiN films of about $1 \mu \mathrm{m}$ on stainless steel, deposited by unbalanced magnetron sputtering on a polycrystalline stainless steel substrate at ground potential and at floating temperature in an $\mathrm{Ar} / \mathrm{N}_{2}$ gas with a pressure of $0.55 \mathrm{~Pa}$ were repeated, but with a target-substrate distance of $10 \mathrm{~cm}$ instead of $13 \mathrm{~cm}$. Again, the influence of an increasing $\mathrm{N}_{2}$-flow on the resulting out-of-plane orientation was investigated. All deposited films were characterised by XRD $\theta / 2 \theta$. The calculated fraction of grains with a specific out-of-plane orientation as a function of the $\mathrm{N}_{2}$-flow is shown in figure V.7.2.
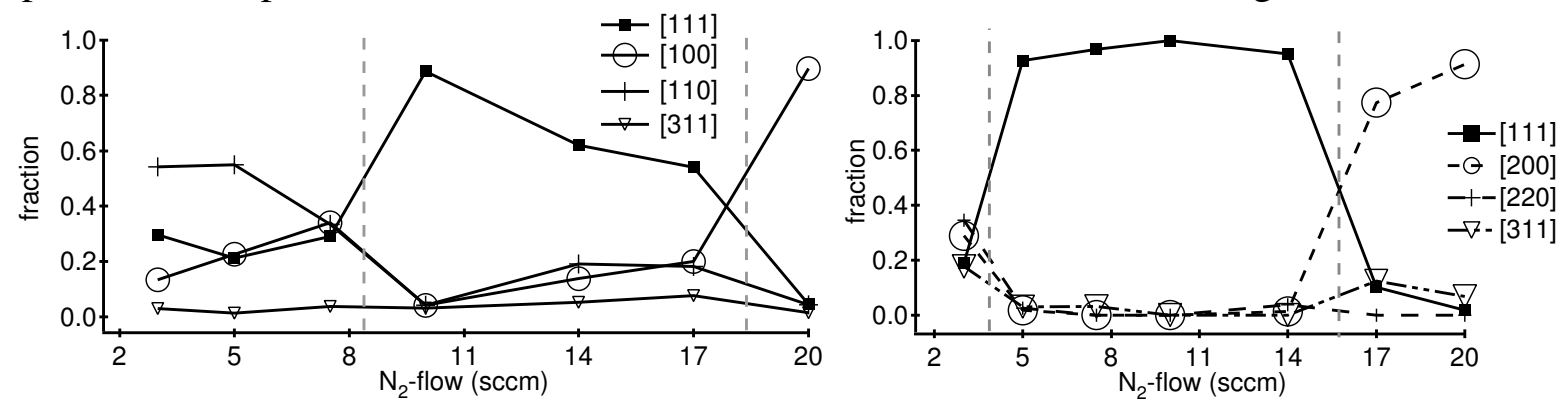

Figure V.7.2: Calculated fraction of grains with a specific out-of-plane orientation in TiN films deposited at a target-substrate distance of $10 \mathrm{~cm}$ as a function of the $\mathrm{N}_{2}$-flow (left). Other depositions conditions were similar to the depositions described in section V.2. For comparison, the results of the depositions at similar conditions but with a T-S distance of $13 \mathrm{~cm}$ are also given (right). 
Similar to the depositions at a target-substrate distance of $13 \mathrm{~cm}$, a transition from random oriented to preferential [111] and finally to [100] out-of-plane oriented films is observed with increasing $\mathrm{N}_{2}$-flow. However, comparing these results with the resulting out-of-plane orientation of the corresponding films deposited at a target-substrate distance of $13 \mathrm{~cm}$ (see fig. V.2.5), it is observed that the transitions from random orientation to a [111] out-of-plane alignment and from a [111] to a [100] out-of-plane alignment are shifted to higher $\mathrm{N}_{2}$-flows. The shift of the transition regions towards higher $\mathrm{N}_{2}$-flow can be explained by an overall decrease of the adparticle mobility when decreasing the target-substrate distance. Although decreasing the target-substrate distance slightly increases the kinetic energy of the sputtered particles and also increases the plasma density, and hence the corresponding energy flux due to the plasma-surface interaction, the overall energy flux per deposited atom decreases because of the stronger increase of the deposition rate. Such a decrease of the energy per deposited particle when decreasing the target-substrate distance has been measured by Ekpe et al. [52].

Hence, decreasing the target-substrate distance decreases the energy flux per deposited particle and consequently shifts the transitions from zone Ic, to zone $\mathrm{T}$ and finally to zone II to higher $\mathrm{N}_{2}$-flows.

Influence of the substrate bias:

Finally also the influence of the substrate bias on the resulting out-of-plane orientation has been investigated by carrying out YSZ depositions on a polycrystalline stainless steel substrate at floating temperature, at a pressure of $0.55 \mathrm{~Pa}$ and an $\mathrm{O}_{2}$-flow of 2.5 and $6.5 \mathrm{sccm}$ at varying substrate bias. The influence of the substrate bias on the resulting out-of-plane orientation is shown in figure V.7.3.
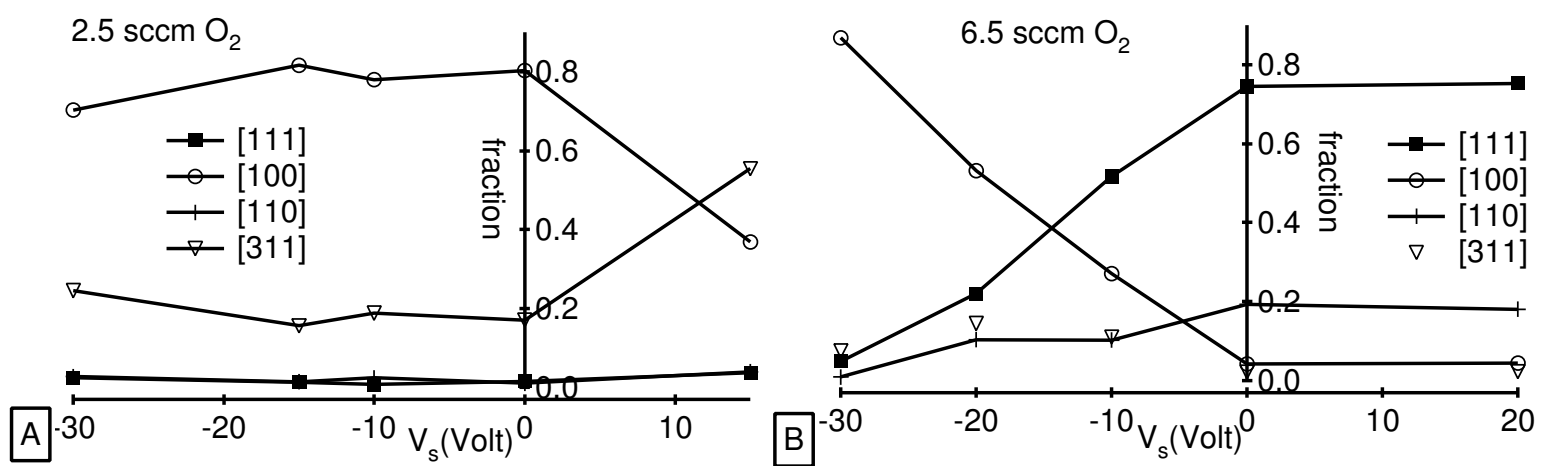

Figure V.7.3: Calculated fraction of grains with a specific out-of-plane orientation in YSZ films deposited on stainless steel at an $\mathrm{O}_{2}$-flow of $2.5 \mathrm{sccm}$ (a) and $6.5 \mathrm{sccm}$ (b) as a function of the substrate bias. Other deposition conditions were similar to the depositions described in section V.3.

As was described in section V.5, the substrate potential $V_{s}$ (with respect to the plasma potential $V_{p}$ ) strongly influences the energy flux towards the growing film. Applying a negative bias will accelerate the positive ions towards the substrate but will repel the electrons. The resulting energy flux will only increase linearly with increasing substrate bias due to an increasing energy of the ions. On the other hand, applying a positive bias will repel the positive ions and will attract all electrons. The energy flux first increases strongly due to the huge amount of electrons that are attracted. Once the substrate potential equals the initial plasma potential, increasing the substrate bias will not further increase the energy flux. This influence of the substrate bias on the energy flux due to the plasma-surface interaction was shown in figure V.5.11.

As was measured by a Langmuir probe and as shown in figure V.5.3, the plasma potential $V_{p}$ is about $-10 \mathrm{~V}$ in steady state during the deposition of $\mathrm{YSZ}$ with an $\mathrm{O}_{2}$-flow of at least $2 \mathrm{sccm}$. 
The negative value of the plasma potential was understood in terms of a disappearing anode. Since YSZ is an electrical isolator, the chamber walls will become also electrical insulating when depositing YSZ on it. As soon as the substrate and the chamber walls are coated with an electrical insulating material, they will disappear as effective anode. Therefore, the plasma potential will lower to negative values. However, at the start of each deposition, a well cleaned metallic and electrical conducting substrate holder (and substrate) are placed in front of the target. Hence, the substrate holder will initially act as an effective anode and the plasma potential will remain slightly positive. As soon as the deposited YSZ film on the substrate holder (and substrate) is thick enough, the substrate holder becomes electrical insulating and the plasma potential in the vicinity of the substrate will drop to a value of $-10 \mathrm{~V}$.

It can be concluded that although YSZ is an electrical insulating material, applying a bias on the substrate can influence the initial film growth.

Let's first discuss the observed influence of a substrate bias for the depositions at an $\mathrm{O}_{2}$-flow of $2.5 \mathrm{sccm}$ (fig. V.7.3.a). As was discussed in section V.3, depositions carried out at similar conditions on a grounded substrate results in a kinetically limited growth with a zone $\mathrm{T}$ structure and a [100] out-of-plane alignment. Hence, applying a negative bias and thus decreasing the energy flux towards the growing film will not really influence the resulting out-of-plane orientation since the deposition was already kinetically limited. Seemingly, applying a negative bias of $-30 \mathrm{~V}$ does not decrease the energy flux that much that a zone Ic structure and a corresponding random out-of-plane orientation is obtained. On the other hand, applying a positive bias increases the energy flux towards the substrate. This should have the same effect on the out-of-plane orientation as increasing the oxygen flow, since this also increases the energy flux (see section V.3). As observed in figure V.7.3.a, a more pronounced fraction of [311] out-of-plane oriented grains is observed for the deposition at a positive bias, which is similar to the out-of-plane orientation of a deposition at 3 à $4 \mathrm{sccm} \mathrm{O}_{2}$ on a grounded substrate (see fig. V.3.5).

Secondly, also the influence of a substrate bias for depositions at high $\mathrm{O}_{2}$-flow was investigated (see fig. V.7.3.b). As was already discussed in section V.3, a deposition on a grounded substrate at $6.5 \mathrm{sccm} \mathrm{O}_{2}$ results already in a mixture of zone $\mathrm{T}$ ([110] out-of-plane oriented) and zone II ([111] out-of-plane oriented) growth. Hence, the deposition is already carried out at an increased adparticle mobility. Therefore, further increasing the adparticle mobility by applying a positive bias will not really influence the resulting out-of-plane orientation, what is observed in figure V.7.3b.

On the other hand, a negative bias will decrease the adparticle mobility. Since the fraction of [111] out-of-plane oriented grains was caused by the aim for lowest surface energy, its contribution should decrease with negative bias what is observed in figure V.7.3.b. Analogous, on should expect that the fraction of [110] out-of-plane oriented grains would increase since the zone T contribution gets more important. However, as was already discussed in section V.6, the [110] out-of-plane oriented grains are not unequivocally faceted and thus cannot overgrow the other grains. Due to the lowered mobility, the fastest growth of the [110] out-of-plane grains will have to compete with other well faceted grains. As seen in figure V.6.15b, the [100] out-of-plane oriented grains will be able to overgrow the [110] grains.

\section{8: Conclusions}

Increasing the reactive gas flow, a strong variation in the microstructure and out-of-plane orientation of YSZ and TiN films was observed. It was shown that this variation is not caused by a possible influence of impurities, but by an increasing mobility during the growth. Also 
the influence of many other deposition parameters, such as target-substrate distance, targetsubstrate angle, $\ldots$ on the microstructure and out-of-plane orientation has been investigated. A model for the resulting out-of-plane orientation and microstructure of magnetron sputtered films has been proposed. This model is mainly based on the fundamental phenomena of structure formation as described in chapter II and summarized in the structure zone model in table II.5. It could be concluded that an increasing adparticle mobility causes a transition from kinetically limited to thermodynamically enhanced growth. This adparticle mobility is related to the energy flux towards the growing film per deposited particle, which could be calculated after measuring the plasma characteristics by a Langmuir probe and simulating the kinetic energy of the sputtered particles.

In case of a thermodynamically enhanced growth, re-structurative grain growth can happen and the resulting preferential out-of-plane orientation corresponds to the plane of lowest surface energy. The microstructure is characterised by a zone II structure.

At lower adparticle mobility, a zone T structure will develop in which the grains aligned with their geometric fastest growing direction along the substrate normal will overgrow the other grains, at least if they are unequivocally faceted. This fastest geometric growth direction is determined by the crystal habit of the grains. On its turn, this crystal habit is formed by the planes of lowest crystallographic growth rate. It was discussed that the anisotropy in crystallographic growth rate depends on the number of nearest neighbours offered between the growing plane and the incoming adparticle. Hence, it is influenced by the state of the adparticles (admolecules or adatoms) and by the termination of the growing plane (which is determined by the state of the reactive gas, i.e. molecular or atomic), which are two parameters that could be measured by energy resolved mass spectrometry.

At still lower mobility, a zone Ic structure develops. Due to the absence of any interaction between the several grains and due to the immobility of the grain boundaries, a random outof-plane orientation of the nuclei is conserved through the whole film growth.

The experimentally observed influence of the reactive gas flow, the target-substrate angle, the target-substrate distance and the substrate bias on the resulting out-of-plane orientation and microstructure of the deposited YSZ and TiN films could be understood by means of the proposed growth mechanism. Also many other, in literature reported, results could be explained by the proposed growth mechanism.

Although the proposed model was only validated for cubic materials, it is believed to be applicable for materials with another structure, e.g. hexagonal, tetragonal,...

\section{References}

1: F.S. Galasso in "structure and properties of inorganic solids" published by Pergamon press ISBN: 0080068731

2: A. Christensen, E.A. Carter: Phys. Rev. B 58(12) (1998) p.8050-8064

3: Powder Diffraction data base of the International Centre for Diffraction data.

4: T.Q. Li, S. Noda, Y. Tsui, T. Ohsawa, H. Komiyama: J. Vac. Sci. Technol. A 20(3) (2002) p.583-588

5: T.Q. Li, S. Noda, H. Komiyama: J. Vac. Sci. Technol. A 21(5) (2003) p.1717-1723

6: R. Banerjee, K. Singh, P. Ayyub, M.K. Totlani, A.K. Suri: J. Vac. Sci. Technol. A 21(1) (2003) p. 310-317

7: M.K. Lee, H. S. Kang, W.W. Kim, J.S. Kim, W.J. Lee: J. Mater. Res. 12 (9) (1997) p.23932400

8: L.J. Meng, M.P. dos Santos: Surf. Coat. Technol. 90 (1997) p.64-70

9: H.Q. Lou, N. Axen, R.E. Somekh, I.M. Hutchings: Surf. Coat. Technol. 90 (1997) p.123127 
10: J.P. Zhao, X. Wang, Z.Y. Chen, S.Q. Yang, T.S. Shi, X.H. Liu: J. Phys. D: Appl. Phys. 30 (1997) p.5-12

11: U.C. Oh, J.H. Je: J. Appl. Phys. 74 (3) (1993) p. 1692-1696

12: J.H. Je, D.Y. Noh, H.K. Kim, K.S. Liang: J. Appl. Phys. 81 (9) (1997) p.6126-6133

13: G. Abadias, Y.Y. Tsee: J. Appl. Phys. 95 (5) (2004) p.2414-2428

14: L. Combadiere, J. Machet: Surf. Coat. Technol. 88 (1996) p.17-27

15: P. Patsalas, C. Chariditis, S. Logothetidis : Surf. Coat. Technol. 125 (2000) p.335-340

16: J.E. Greene, J.E. Sundgren, L. Hultman, I. Petrov, D.B. Bergstrom : Appl. Phys. Lett. 67 (20) (1995) p.2928-2930

17: L. Hultman, J.E. Sundgren, J.E. Greene, D.B. Bergstrom, I. Petrov: J. Appl. Phys. 78 (9) (1995) p.5395-5403

18: I. Petrov, F. Adibi, J.E. Greene, L. Hultman, J.E. Sundgren: Appl. Phys. Lett. 63 (1) (1993) p.36-38

19: B. Rauschenbach, J. W. Gerlach: Cryst. Res. Technol. 35 (6-7) (2000) p.675-688

20: J.W. Gerlach, U. Preckwinkel, H. Wengenmair, T. Kraus, B. Rauschenbach: Appl. Phys. Lett. 68 (17) (1996) p.2360-2362

21: M. Zeilter, J.W. Gerlach, T. Kraus, B. Rauschenbach: Appl. Phys. Lett. 70 (10) (1997) p.1254-1256

22: H. Holzschuh, H. Suhr: Appl. Phys. Lett. 59 (1991) p.470-472

23: J. Pelleg, L.Z. Zevin, S. Lungo: Thin Solid Films 197 (1991) p.117-128

24: Y. Iijima, M. Hosaka, N. Tanabe, N. Sadakata, T. Saitoh, O. Kohno: J. Mater. Res. 13(11) (1998) p.3106-3113

25: K.G. Ressler, N. Sonnenberg, M.J. Cima: J. Am. Ceram. Soc. 80(10) (1997) p.2637-2648

26: L. Dong, D.J. Srolovitz: J. Appl. Phys. 84(9) (1998) p. 5261-5269

27: I. Petrov, L. Hultman, J.E. Sundgren, J.E. Greene: J. Vac. Sci. Technol. A 10 (2) (1992) p.265-272

28: I. Petrov, F. Adibi, J.E. Greene, L. Hultman, J.E. Sundgren: Appl. Phys. Lett. 63(1)

(1993) p.36-38

29: V. Vancoppenolle, P.Y. Jouan, M. Wautelet, J.P. Dauchot, M. Hecq: J. vac. Sci. Technol.

A 17(6) (1999) p.3317-3321

30: B. Chapman in "Glow Discharge Processes" published by John Wiley \& sons ISBN: 0

$47107828 \mathrm{X}$

31: Stopping Range of Ions in Matter version 2003.20, www.srim.org

32: T.P. Drüsedau, T. Bock, T.-M. John, F. Klabunde, W. Eckstein: J. Vac. Sci. Technol. A 17(5) (1999) p.2896-2905

33: S.D. Ekpe, S.K. Drew: J. Vac. Sci. Technol. A 20(6) (2002) p.1877-1885

34: S.D. Ekpe, S.K. Drew: J. Vac. Sci. Technol. A 21(2) (2003) p.476-483

35: H. Kersten, H. Deutsch, H. Steffen, G.M.W. Kroesen, R. Hippler: Vacuum 63 (2001) p.385-431

36: National Institute of Standards and Technology: Chemistry webbook, available on http://webbook.nist.gov/chemistry

37: Lange's Handbook of Chemistry $\left(13^{\text {th }}\right.$ ed.) 1985 edited by J.A. Dean, published by McGraw-Hill Inc. ISBN: 0070161925

38: W. Westwood in "Sputter deposition" published by AVS, ISBN: 0735401055

39: C.S. Shin, D. Gall, Y.K. Kim, N. Hellgren, I. Petrov, J.E. Greene: J. Appl. Phys. 92(9 (2002) p.5084-5093

40: R . Snyders, R. Gouttebaron, J.P. Dauchot, M. Hecq: J. Anal. At. Spectrum. 18 (2003) p.618-623

41: S. Konstantinidis, A. Ricard, R. Snyders, H. Vandeparre, J.P. Dauchot, M. Hecq : Surf. Coat. Technol. 200 (2005) p.841-845 
42: National Institute of Standards and Technology (NIST), Electron-impact cross-sections for ionization, available on http://physics.nist.gov

43: S. Mahieu, P. Ghekiere, G. De Winter, D. Depla, R. De Gryse, O.I. Lebedev, G. Van Tendeloo: Thin Solid Films 484 (2005) p.18-25

44: J.A. Thornton: J. Vac. Sci. Technol (15) (1978) p.188-192

45: D. Gall, S. Kodambaka, M.A. Wall, I. Petrov, J.E. Greene: J. Appl. Phys. 93(11) (2003) p.9086-9094

46: H. Huang, G.H. Gilmer: J. Appl. Phys. 84 (1998) p.3636-3649

47: F. Adibi, I. Petrov, L. Hultman, U. Wahlström, T. Shimizu, D. McIntyre, J.E. Greene, J.E. Sundgren: J. Appl. Phys. 69(9 (1991) p.6437-9449

48: K. Wada, N.Yamaguchi, H. Matsubara: Surf. Coat. Technol. 184 (2004) p.55-62

49: L. Abelmann, C. Lodder: Thin Solid Films 305 (1997) p.1-21

50: J. Kools: J. Vac. Sci. Technol. A23(1) (2005) p. 85-89

51: L. Dong, R.W. Smith, D.J. Srolovitz: J. Appl. Phys. 80(10) (1996) p.5682-5690

52: S.D. Ekpe, S.K. Dew: J. Vac. Sci. Technol. A 22(4) (2004) p.1420-1424 


\section{Appendix: Calculation of the electron and ion energy flux.}

To calculate the electron and ion energy flux towards the substrate, the flux of electrons $\Theta_{\mathrm{e}}$ and ions $\Theta_{\mathrm{i}}$ should be known together with the energy of these particles $\varepsilon_{\mathrm{e}}$ or $\varepsilon_{\mathrm{i}}$. If this $\varepsilon_{\mathrm{e}}$ and $\varepsilon_{\mathrm{i}}$ are an average of the energy of all particles arriving on the substrate, the total energy flux is given by $\left(\Theta_{\mathrm{e}} \mathrm{x} \varepsilon_{\mathrm{e}}\right)+\left(\Theta_{\mathrm{i}} \mathrm{x} \mathcal{\varepsilon}_{\mathrm{i}}\right)$.

The flux towards the substrate: $\Theta_{\underline{e}}$ and $\Theta_{\mathrm{i}}$

This flux is given by the expression

$\Theta_{e}=\frac{P_{e}}{\sqrt{2 \pi M_{e} k T_{e}}}=\frac{n_{e} k T_{e}}{\sqrt{2 \pi M_{e} k T_{e}}}$ because $P_{e}=n_{e} k T_{e}$

$=\frac{n_{e} \sqrt{k T_{e}}}{\sqrt{2 \pi M_{e}}}=\frac{n_{e} \sqrt{\pi\langle E\rangle / 4}}{\sqrt{2 \pi M_{e}}}$ because $\langle E\rangle=\frac{M_{e}\langle v\rangle^{2}}{2}=\frac{M_{e}}{2} \frac{8 k T_{e}}{\pi M_{e}}=\frac{4 k T_{e}}{\pi}$ (Maxwell - Boltzmann $)$

$=\frac{n_{e} \sqrt{\langle E\rangle}}{\sqrt{8 M_{e}}}$

With $\langle E\rangle$ the average electron energy and $n_{e}$ the electron density as measured by the Langmuir probe.

The same rules for the ion flux: $\Theta_{i}=\frac{n_{i} \sqrt{\langle E\rangle}}{\sqrt{8 M i}}$ with $n_{i}$ the ion density measured by the Langmuir probe.

The average energy of the electrons and ions: $\mathscr{E}_{\underline{e}}$ and $\mathscr{\complement}_{i}$

The Maxwell-Boltzmann distribution expresses the energy distribution of the electrons:

$f(E) d E=A \sqrt{E} e^{-E / k T_{e}} d E$

$k T_{e}$ is determined by the equation $\langle E\rangle=\frac{\int_{0}^{\infty} A \sqrt{E} E e^{-E / k T_{e}} d E}{\int_{0}^{\infty} A \sqrt{E} e^{-E / k T_{e}} d E}$ with $\langle E\rangle$ the average electron energy as

measured with the Langmuir probe.

$A$ is used to normalize the distribution: $\int_{0}^{\infty} A \sqrt{E} e^{-E / k T_{e}} d E=1$

For the calculation of all these integrals, not infinity was used as the upper limit, but a sufficient large finite value, i.e. $+10 \mathrm{eV}$.

Knowing the energy distribution, the energy $\mathscr{E}_{\mathrm{e}}$ is expressed by:

$\mathscr{E}_{\mathrm{e}}=\int_{\left(V_{p}-V\right)}^{10} A \sqrt{E}\left(E-\left|V_{p}-V\right|\right) e^{-E / k T_{e}} d E$ for $\left(V_{p}>V\right)$

$\varepsilon_{\mathrm{e}}=\int_{0}^{10} A \sqrt{E} E e^{-E / k T_{e}} d E$ for $\left(V_{p}<V\right)$

The ion energy $\mathscr{C}_{\mathrm{i}}$ is approximated by:

$\varepsilon_{\mathrm{i}}=0$ for $\left(V_{p}<V\right)$

$\mathscr{E}_{\mathrm{i}}=\left(\langle E\rangle+\left|V_{p}-V\right|\right)$ for $\left(V_{p}>V\right)$ 


\section{Chapter VI: Mechanism of in-plane alignment.}

\section{1: Introduction}

In the previous chapter, the development of the crystallographic out-of-plane orientation was discussed. It was shown that the microstructure as well as the crystallographic out-of-plane orientation can be understood as a function of the elementary process of diffusion and the basic phenomena of structure formation, i.e. nucleation, crystal growth and re-structurative grain growth. It was also concluded that only in zone $\mathrm{T}$ and zone II a crystallographic preferential out-of-plane alignment directly related to the deposition conditions will develop. As discussed in chapter IV, such preferential out-of-plane aligned thin films can also have a crystallographic in-plane alignment. Since the scope of this thesis is to study the biaxial alignment in thin films deposited by magnetron sputtering, the conditions under which a biaxial alignment develops were investigated.

First the experimental results obtained in our group will be discussed. The development of a biaxial alignment was observed when tilting the substrate with respect to the material flux, i.e. inclined substrate deposition (ISD). Therefore, the influence of that substrate tilt on the crystallographic alignment was studied.

Since also a lot of other research groups observed a biaxial alignment in their films deposited by ISD, their observations will be summarized.

Also a model for the development of the in-plane alignment will be proposed (VI.3). Finally the two already in literature existing models will be discussed.

\section{2: Experimental observations}

Again, TiN and YSZ thin films were deposited with the system described in chapter III. A Ti or a $\mathrm{Zr} / \mathrm{Y}(55 / 45 \mathrm{Wt} \%)$ target was sputtered with an unbalanced magnetron in an $\mathrm{Ar} / \mathrm{N}_{2}$ or an $\mathrm{Ar} / \mathrm{O}_{2}$ mixture on polycrystalline stainless steel and glass substrates. The deposited thin films were about $1 \mu \mathrm{m}$ thick and ex-situ characterised by XRD $\theta / 2 \theta$, XRD pole figures, SEM and TEM.

\section{TiN:}

Two TiN films were deposited at an $\mathrm{Ar} / \mathrm{N}_{2}$ ratio of 55/5, a target-substrate distance of $9.5 \mathrm{~cm}$, a pressure of $0.48 \mathrm{~Pa}$, a substrate bias of $+15 \mathrm{~V}$ and a discharge current of $0.9 \mathrm{~A}$. The first was deposited on a non-tilted substrate $\left(\alpha=0^{\circ}\right)$, while the second was deposited on a substrate with tilt angle $\alpha=65^{\circ}$, in which $\alpha$ is defined as the angle between the substrate normal and the target normal. Figure VI.2.1 shows the XRD $\theta / 2 \theta$ spectra of both films. It is observed that both TiN films have a clear preferential [111] out-of-plane alignment.
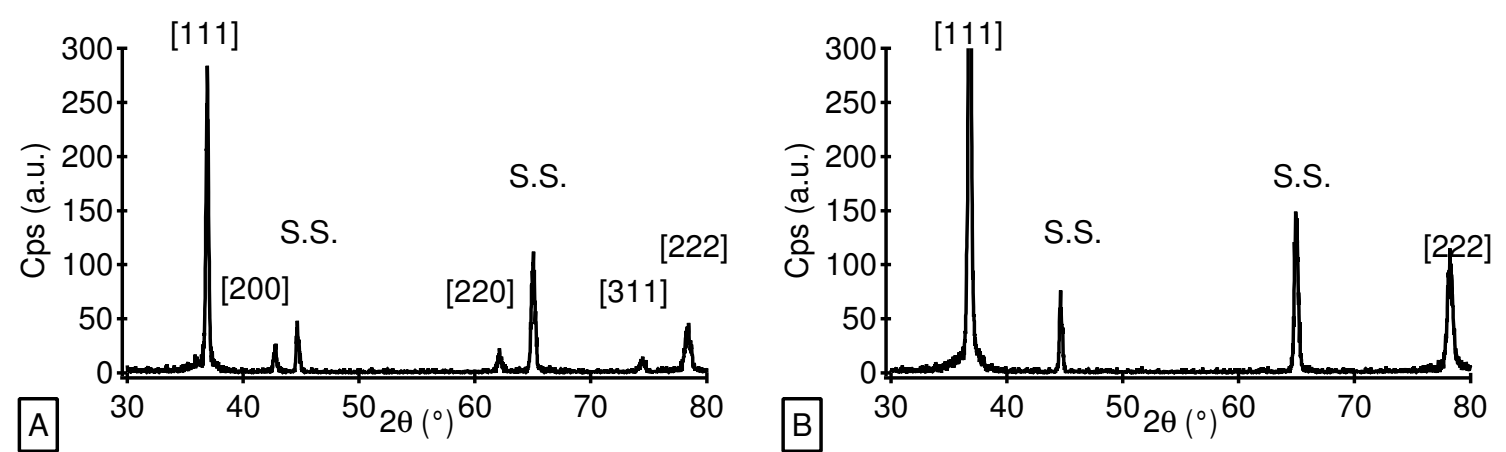

Figure VI.2.1: XRD $\theta / 2 \theta$ spectrum of a TiN film deposited on stainless steel with $\mathrm{Ar} / \mathrm{N}_{2}=55 / 5, \mathrm{P}=0.48 \mathrm{~Pa}, \mathrm{~V}_{\mathrm{s}}=$ $+15 \mathrm{~V}, \mathrm{~T}-\mathrm{S}=9.5 \mathrm{~cm}, \mathrm{I}_{\mathrm{d}}=0.9 \mathrm{~A}$ and $\alpha=0^{\circ}$ (a) or $65^{\circ}$ (b). Both have a clear [111] out-of-plane alignment. 
Plan view SEM images are shown in figure VI.2.2. It is observed that both TiN films have a clear faceted structure. All grains are oriented with their top upwards, what corresponds with a $\{100\}$ faceted crystal habit. It is also observed that the faceted grains of the TiN film deposited on a non-tilted substrate has a random microstructural in-plane orientation. However, the TiN film deposited on a tilted substrate has a clear microstructural in-plane alignment, in which the triangular shaped grains are all oriented with a side towards the incoming material flux, which is indicated by the arrow. This correlation between the in-plane alignment and the incoming material flux is schematically drawn in figure VI.2.3.

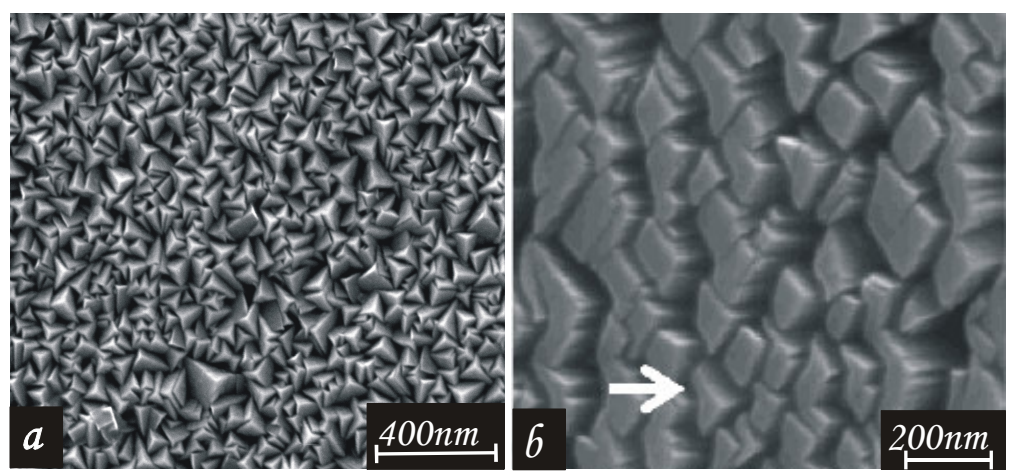

Figure VI.2.2: $\mathrm{SEM}$ plan view of a TiN film deposited on stainless steel with $\mathrm{Ar} / \mathrm{N}_{2}=55 / 5, \mathrm{P}=0.48 \mathrm{~Pa}, \mathrm{~V}_{\mathrm{s}}=$ $+15 \mathrm{~V}, \mathrm{~T}-\mathrm{S}=9.5 \mathrm{~cm}, \mathrm{I}_{\mathrm{d}}=0.9 \mathrm{~A}$ and $\alpha=0^{\circ}$ (a) or $65^{\circ}$ (b). Both have a clear faceted structure. The arrow indicates the orthogonal projection of the incoming material flux.

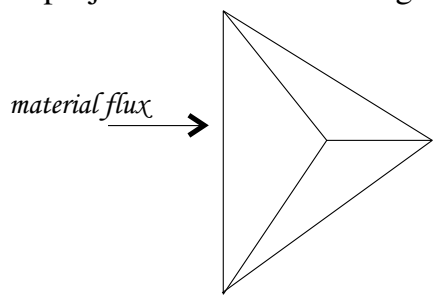

Figure VI.2.3: Schematic drawing of the correlation between the microstructural in-plane alignment and the incoming material flux, which is indicated by the arrow.

The crystallographic in-plane alignment can be measured by pole figures, as discussed in chapter IV. The $\{200\}$ pole figures, i.e. a stereographic projection of the experimentally measured direction of the $<200>$ orientations, are shown in figure VI.2.4. A diffraction ring, and thus a random in-plane alignment is observed for the deposition on a non-tilted substrate. Tilting the substrate causes the appearance of clear diffraction spots instead of a diffraction ring, indicating the development of an in-plane alignment. The diffraction peaks are all nearly positioned at $\mathrm{Chi}=55^{\circ}$, what corresponds with the [111] out-of-plane alignment since the angle between a [002] and [111] direction is $55^{\circ}$. It is also observed that the grains faces with a (200) plane towards the incoming material flux, of which the orthogonal projection is indicated by the arrow. A schematic drawing of the correlation between the crystallographic in-plane alignment and the direction of the incoming material flux is drawn in figure VI.2.5.
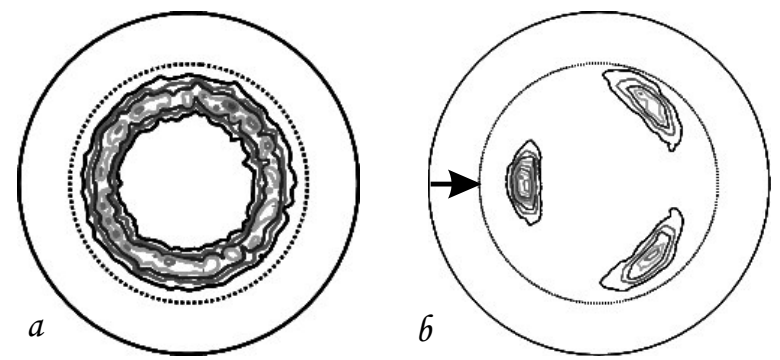

Figure VI.2.4: $\{200\}$ pole figures of a TiN film deposited on stainless steel with $\mathrm{Ar} / \mathrm{N}_{2}=55 / 5, \mathrm{P}=0.48 \mathrm{~Pa}, \mathrm{~V}_{\mathrm{s}}=$ $+15 \mathrm{~V}, \mathrm{~T}-\mathrm{S}=9.5 \mathrm{~cm}, \mathrm{I}_{\mathrm{d}}=0.9 \mathrm{~A}$ and $\alpha=0^{\circ}$ (a) or $65^{\circ}$ (b). The arrow indicates the orthogonal projection of the incoming material flux. 


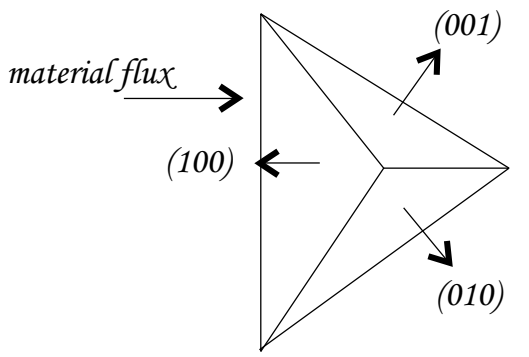

Figure VI.2.5: Schematic drawing of the correlation between the crystallographic in-plane alignment and the incoming material flux, which is indicated by the arrow.

Figure VI.2.6 are TEM cross-section of the TiN films deposited on glass. In both cases, an evolutionary overgrowth is observed. In case of the TiN film deposited on a non-tilted substrate, the columns are perpendicular to the substrate while they are about $15^{\circ}$ tilted towards the incoming material flux for the TiN film deposited on the tilted substrate.

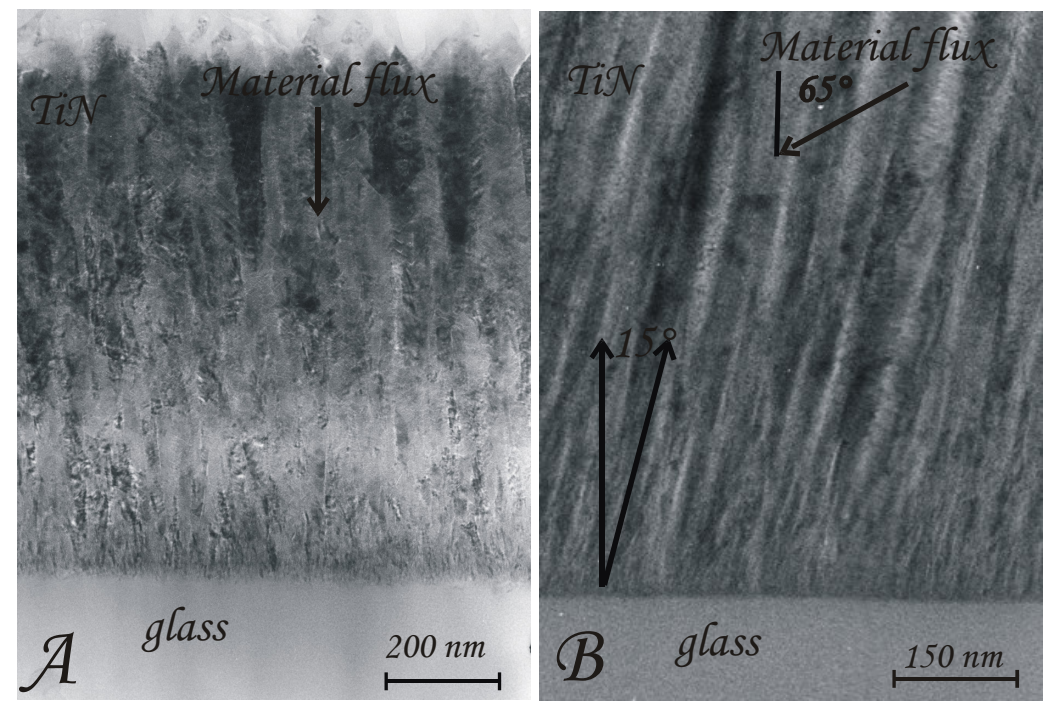

Figure VI.2.6: TEM cross-sections of a TiN film deposited on glass with $\mathrm{Ar} / \mathrm{N}_{2}=55 / 5, \mathrm{P}=0.48 \mathrm{~Pa}, \mathrm{~V}_{\mathrm{s}}=+15 \mathrm{~V}$, $\mathrm{T}-\mathrm{S}=9.5 \mathrm{~cm}, \mathrm{I}_{\mathrm{d}}=0.9 \mathrm{~A}$ and $\alpha=0^{\circ}$ (a) or $65^{\circ}(\mathrm{b})$. Both have a clear columnar structure. The arrow indicates the incoming material flux.

Comparing the experimental results of the deposition on a non-tilted and on a tilted substrate, it can be concluded that tilting the substrate only causes the development of an in-plane alignment, while mainly conserving the out-of-plane orientation and the microstructure. As observed in the SEM and TEM images, both films have a clear zone T structure, since they are characterised by an evolutionary overgrowth resulting in nicely faceted columns. As described in chapter $\mathrm{V}$, the resulting out-of-plane orientation and the crystal habit for depositions in zone $\mathrm{T}$ conditions depend on the plasma composition. According to table V.6.6, a [111] out-of-plane alignment and $\{100\}$ crystal habit should develop, because it was measured by mass-spectrometry that the adparticles are $\mathrm{Ti}$ adatoms and that the reactive gas acts as $\mathrm{N}_{2}$ molecules. The [111] out-of-plane alignment as well as the $\{100\}$ crystal habit are clearly observed in the $\theta / 2 \theta$ spectra and SEM plan views respectively. Comparing the schematic drawing of figure VI.2.3 and VI.2.5, it is observed that the microstructural and the crystallographic in-plane alignment correspond with each other.

All other biaxially aligned TiN films deposited with this technique also exhibit a clear out-ofplane alignment and faceted column tops. Hence, it could be concluded that all biaxially aligned TiN thin films were deposited in zone T conditions. 


\section{YSZ:}

A similar investigation to the influence of a substrate tilt on the film characteristics was carried out for YSZ depositions. Again, two films of about $1 \mu \mathrm{m}$ were deposited at an $\mathrm{Ar} / \mathrm{O}_{2}$ ratio of 58/2, a target-substrate distance of $13 \mathrm{~cm}$, a pressure of $0.55 \mathrm{~Pa}$, a grounded stainless steel substrate and a discharge current of $0.9 \mathrm{~A}$. The first was deposited on a non-tilted substrate $\left(\alpha=0^{\circ}\right)$, while the second was deposited on a substrate with tilt angle $\alpha=55^{\circ}$. Again, the deposited films were characterised by XRD $\theta / 2 \theta$, pole figures, SEM and TEM. The XRD $\theta / 2 \theta$ spectra, as shown in figure VI.2.7, indicate that both YSZ films have a nearly perfect [100] out-of-plane alignment.
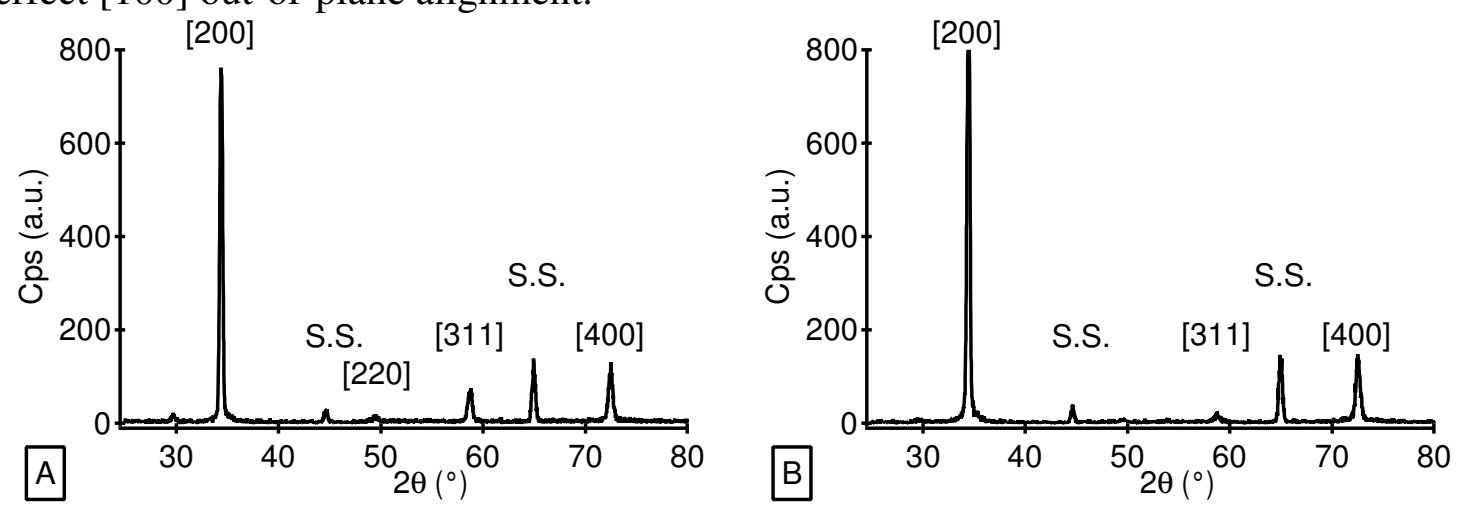

Figure VI.2.7: XRD $\theta / 2 \theta$ spectrum of YSZ films deposited on stainless steel with $\mathrm{Ar} / \mathrm{O}_{2}=58 / 2, \mathrm{P}=0.55 \mathrm{~Pa}, \mathrm{~V}_{\mathrm{s}}=$ $0 \mathrm{~V}, \mathrm{~T}-\mathrm{S}=13 \mathrm{~cm}, \mathrm{I}_{\mathrm{d}}=0.9 \mathrm{~A}$ and $\alpha=0^{\circ}$ (a) or $55^{\circ}(\mathrm{b})$. Both have a clear [100] out-of-plane alignment.

The $\{111\}$ pole figures indicate that the film deposited on a non-tilted substrate has a random in-plane alignment, while tilting the substrate causes again a clear in-plane alignment.
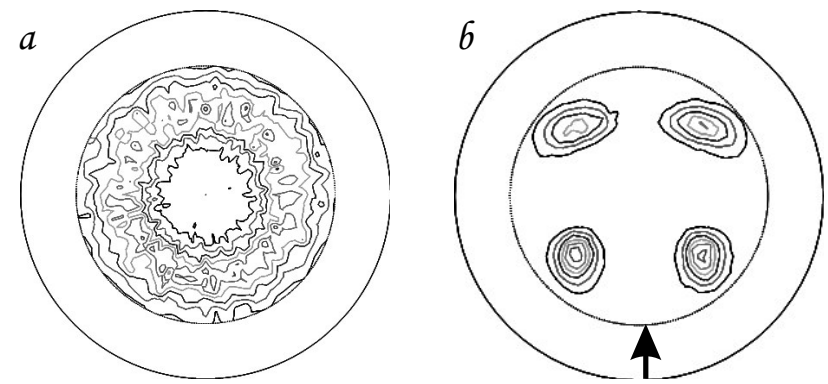

Figure VI.2.8: $\{111\}$ pole figures of YSZ films deposited on stainless steel with $\mathrm{Ar} / \mathrm{O}_{2}=58 / 2, \mathrm{P}=0.55 \mathrm{~Pa}, \mathrm{~V}_{\mathrm{s}}=$ $0 \mathrm{~V}, \mathrm{~T}-\mathrm{S}=13 \mathrm{~cm}, \mathrm{I}_{\mathrm{d}}=0.9 \mathrm{~A}$ and $\alpha=0^{\circ}$ (a) or $55^{\circ}(\mathrm{b})$. The orthogonal projection of the incoming material flux is indicated by the arrow.

A SEM plan view of the YSZ film deposited on the tilted substrate is shown in figure VI.2.9. It is clearly seen that the grains are well faceted and have a square shape as seen in plan view. All grains point with a corner instead of a side towards the incoming material flux of which the orthogonal projection is indicated by the arrow.

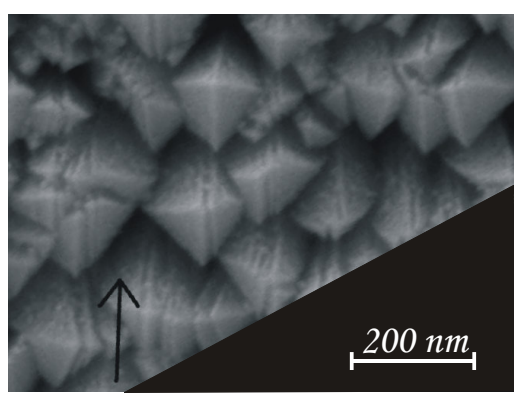

Figure VI.2.9: SEM plan view of the YSZ film deposited on stainless steel on a tilted substrate. The orthogonal projection of the incoming material flux is indicated by the arrow. All grains are well faceted and nicely aligned with their top towards the material flux. 
Figure VI.2.10 shows a TEM cross-section of the YSZ film deposited on a tilted substrate. A clear evolutionary overgrowth, resulting in faceted columns is observed. The columns are tilted towards the incoming material flux.

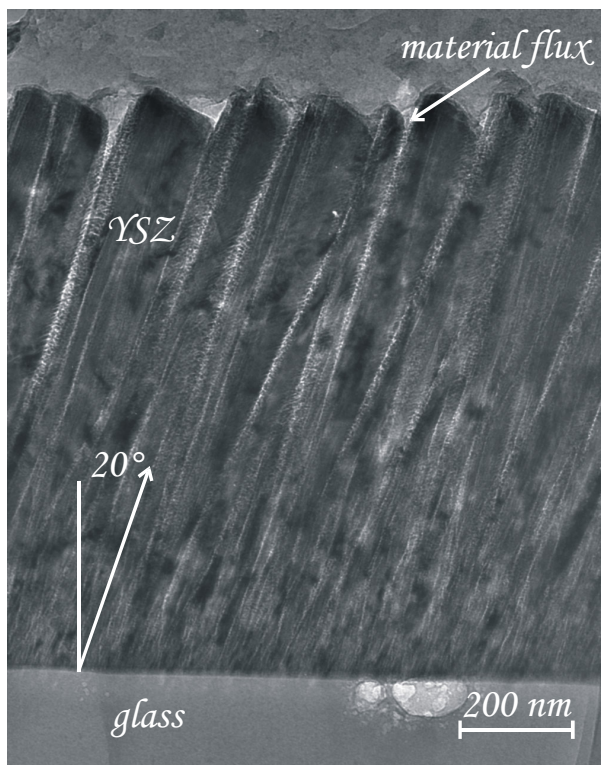

Figure VI.2.10: TEM cross-sections of YSZ films deposited on glass with $\mathrm{Ar} / \mathrm{O}_{2}=58 / 2, \mathrm{P}=0.55 \mathrm{~Pa}, \mathrm{~V}_{\mathrm{s}}=0 \mathrm{~V}, \mathrm{~T}$ $\mathrm{S}=13 \mathrm{~cm}, \mathrm{I}_{\mathrm{d}}=0.9 \mathrm{~A}$ and $\alpha=55^{\circ}$. The incoming material flux is indicated by the arrow.

Well faceted columns are observed.

Comparing the experimental results of the YSZ film deposited on a tilted and on a non-tilted substrate, it is again observed that tilting the substrate causes an in-plane alignment, while maintaining the global microstructure and the out-of-plane alignment.

Since a nearly perfect out-of-plane alignment and an evolutionary overgrowth resulting in faceted columns is observed, both films are deposited in zone T conditions. Moreover, the experimental results, i.e. a [100] out-of-plane alignment and the $\{111\}$ crystal habit, are in accordance with the conclusions of tableV.6.6 since mass-spectrometry indicates that the deposition is carried out with $\mathrm{Zr} / \mathrm{Y}$ adatoms and molecular $\mathrm{O}_{2}$ gas. A schematic drawing of the $\{111\}$ crystal habit is shown in figure VI.2.11. It is observed that this crystal habit corresponds with the SEM plan view in figure VI.2.9 and with the pole figure in figure VI.2.8b.

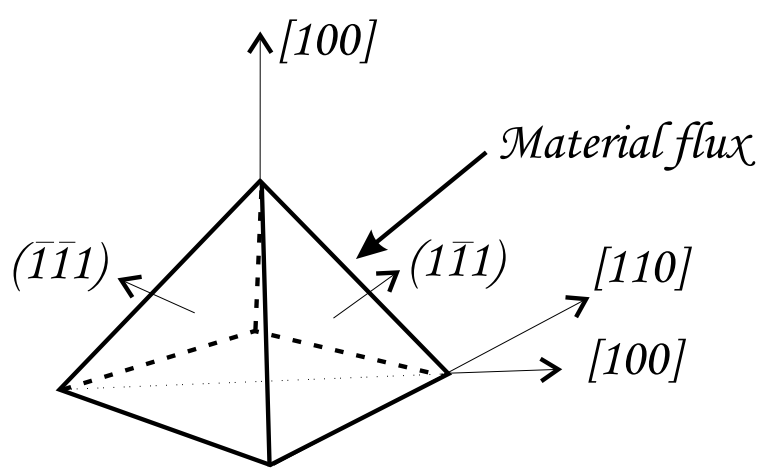

Figure VI.2.11: Schematic drawing of $\{111\}$ crystal habit, and the observed alignment towards the incoming material flux, indicated by the arrow.

Again, all other biaxially aligned YSZ films deposited with this technique exhibit a clear outof-plane alignment and faceted column tops. Hence, it could be concluded that all biaxially aligned YSZ thin films were deposited in zone T conditions. 


\section{Other materials:}

Beside TiN and YSZ, also biaxially aligned Indium Tin Oxide [1], Magnesium Oxide [2] and Chromium [3] were deposited. The biaxially aligned $\mathrm{MgO}$ and $\mathrm{Cr}$ were deposited and investigated by my colleague Pieter Ghekiere. A detailed description of his work will be published in his thesis [4].

For all materials, it was observed that most biaxially aligned thin films exhibit a clear zone $\mathrm{T}$ structure, i.e. a nearly perfect out-of-plane alignment and faceted columns.

\section{Other research groups:}

It can be concluded that an in-plane alignment, and hence biaxially aligned thin films, can be obtained by only tilting the substrate with respect to the material flux. In literature, this technique is called Inclined Substrate Deposition (ISD) and has been used by many researchers. Table VI.2.12 summarizes the reported biaxially aligned thin films in literature. The deposited material, the used deposition technique and the references are given.

Table VI.2.12: Some biaxially aligned thin films obtained by inclined substrate deposition as reported in literature.

\begin{tabular}{|l|l|l|l|}
\hline First author & material & Deposition technique & references \\
\hline Karpenko & $\mathrm{Mo}$ & Magnetron sputtering & 5 \\
\hline Bauer & $\mathrm{CeO}_{2}, \mathrm{YSZ}, \mathrm{MgO}$ & e-beam evaporation & 6 \\
\hline Mancini & $\mathrm{CeO}_{2}$ & e-beam evaporation & 7 \\
\hline Janssens & $\mathrm{Cr}$ & Magnetron sputtering & 8 \\
\hline Hashimoto & $\mathrm{Al}$ & Thermal evaporation & 9 \\
\hline Rodriguez-Navarro & $\mathrm{AlN}$ & Magnetron sputtering & 10 \\
\hline Itoh & $\mathrm{Co}$ & Magnetron sputtering & 11 \\
\hline Hasegawa & $\mathrm{YSZ}$ & Pulsed Laser Deposition & 12 \\
\hline Hasegawa & $\mathrm{MgO}$ & e-beam evaporation & 13 \\
\hline Quinton & $\mathrm{YSZ}$ & Pulsed Laser Deposition & 14 \\
\hline Chudzik & $\mathrm{MgO}$ & e-beam evaporation & 15 \\
\hline Ma & $\mathrm{MgO}$ & e-beam evaporation & 16 \\
\hline Koritala & $\mathrm{MgO}$ & e-beam evaporation & 17 \\
\hline Sato & $\mathrm{YSZ}$ & Pulsed Laser Deposition & 18 \\
\hline
\end{tabular}

Although the numerous reports of biaxially aligned thin films deposited by inclined substrate deposition, only two major models explaining the resulting in-plane alignment has been found. These two models will be discussed in section VI.4. First an alternative model will be discussed based on the experimental results discussed in VI.2.

\section{3: Proposed model for the in-plane alignment}

From the experimental observations of our own depositions, it could be concluded that all biaxially aligned thin films have a zone $\mathrm{T}$ structure, i.e. a clear preferential out-of-plane alignment and faceted column tops.

Hence, before proposing a model for the in-plane alignment, the zone $\mathrm{T}$ growth should be discussed in more detail.

It was already discussed in chapter II that the adparticle mobility in zone T conditions is high enough to allow nucleation into crystalline islands. These crystalline islands will expand due to the capture and incorporation of incoming adparticles.

These captured adparticles can come from the gas phase by direct incorporation into the expanding island. This growth is called the 3D growth and is shown in figure VI.3.1a-e. 
However, due to the rather high mobility of the adparticles, the expansion of the island can also happen by incorporation of adparticles which first stroke on the bare substrate and then diffused towards the expanding island. This growth is called a 2D growth and is shown in figure VI.3.1a. Since also the adparticle diffusion from one grain to another grain is allowed, this 2D growth can also originate from adparticles which first hit another grain, diffuses off that grain and finally get incorporated into another expanding grain. This kind of 2D growth is shown in figure VI.3.1b.

Due to the allowed adparticle diffusion from one grain to another grain, the neighbouring islands/grains will interact and thus compete with each other. It was discussed in chapter II that the grains with the most tilted facets will envelop the other grains. These grains with the most tilted facets correspond to the grains with the geometric fastest growing direction perpendicular to the substrate and are represented by the light coloured grains in figure VI.3.1. At a given film thickness, this overgrowth is finished what also means that the out-of-plane alignment is completed (see figure VI.3.1d). From that moment on, the grains are only able to further expand by direct capture and incorporation of adparticles, i.e. 3D growth. Finally, a columnar structure with faceted tops develops.

(a)
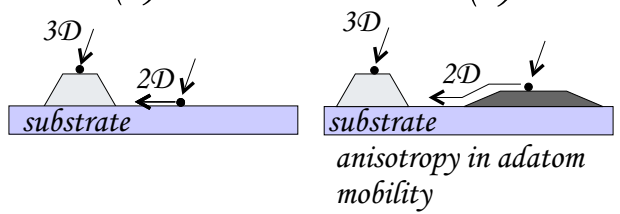

(c)

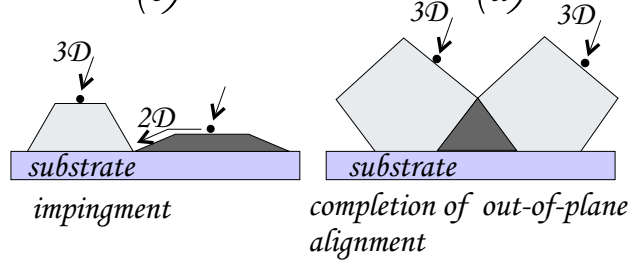

(e)

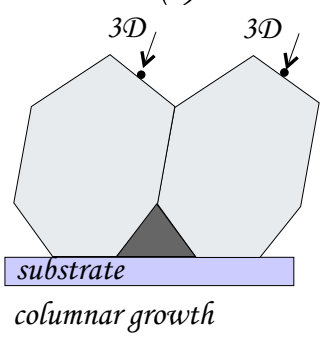

Figure VI.3.1: Different growth phases during a zone T growth. Nucleation of crystalline islands (a). Expansion due to 2D and 3D growth (a- d). Difference in adparticle mobility of different out-of-plane oriented grains (b,c). Interaction and competition between neighbouring grains (c,d). Completion of the out-of-plane alignment (d). Further evolution to a columnar structure due to 3D growth (e).

Hence, before a model for the in-plane alignment can be proposed, it should first be known if the in-plane alignment is caused by the $2 \mathrm{D}$ growth, the $3 \mathrm{D}$ growth or by both growth modes.

\section{VI.3.1: Two-stepped depositions:}

By carrying out specific depositions, one can estimate the importance of the $2 \mathrm{D}$ growth mode for the development of the in-plane alignment. The idea of these depositions is shown in figure VI.3.2. 


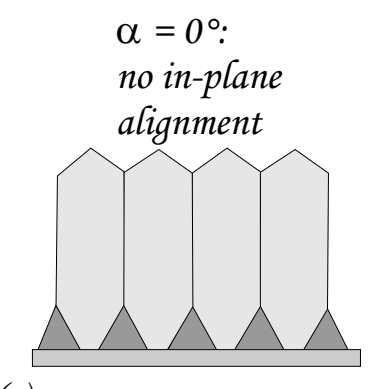

(a)

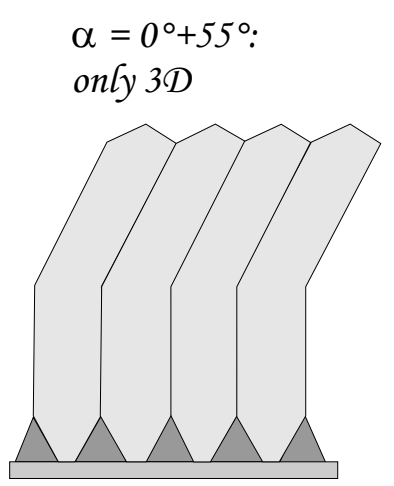

(c)

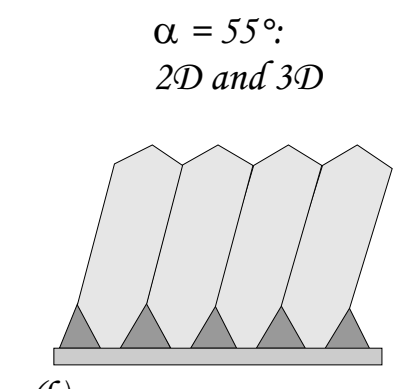

(6)

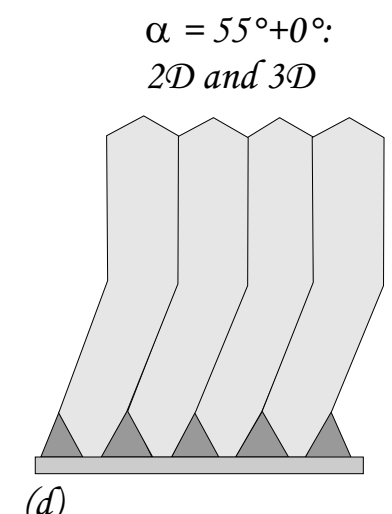

Figure VI.3.2: Schematic drawing of the one- and two-stepped depositions carried out in order to investigate the relative contribution of the $2 \mathrm{D}$ growth on the in-plane alignment.

It was already discussed in section VI.2 that a preferential out-of-plane aligned, but random in-plane oriented thin film develops when the thin film is deposited on a non-tilted substrate (figure VI.3.2a). In case of a deposition on a tilted substrate an in-plane alignment develops (see VI.2). That in-plane alignment can develop due to 2D and 3D growth (figure VI.3.2b) since the islands can expand due to $2 \mathrm{D}$ and $3 \mathrm{D}$ growth.

However, if the deposition is started on a non-tilted substrate and the substrate is only tilted with respect to the material flux after the completion of the out-of-plane alignment, a possible in-plane alignment can only be caused by the 3D growth (figure VI.3.2c). This because the out-of-plane alignment and thus also the $2 \mathrm{D}$ growth is already finished before the substrate is inclined.

Of course, this two-stepped deposition needs a sudden change of the substrate tilt angle during the deposition. In order to be sure that this sudden tilt doesn't has any negative effect on the in-plane alignment, also the inverse two-stepped deposition should be carried out. This means that the deposition is started on a tilted substrate, enabling the development of an in-plane alignment due to 2D and 3D growth, and then tilting the substrate again to the non-tilted situation (figure VI.3.2d).

Comparing both two-stepped depositions (figure VI.3.2c and d) learns how important the 2D growth is for the development of the in-plane alignment. This because a possible in-plane alignment in deposition $\mathrm{C}$ is caused by $3 \mathrm{D}$ growth only, while in deposition $\mathrm{D}$ it can be caused by $2 \mathrm{D}$ and $3 \mathrm{D}$ growth.

These four depositions has been carried out for TiN as well as for YSZ.

- TiN results:

Four TiN films were deposited, according to the above described method. A target-substrate distance of $9.5 \mathrm{~cm}$, a pressure of $0.48 \mathrm{~Pa}, \mathrm{a}+15 \mathrm{~V}$ biased stainless steel substrate, an $\mathrm{Ar} / \mathrm{N}_{2}$ ratio of 55/5 and a discharge current of $0.9 \mathrm{~A}$ was used. The first deposition (see table VI.3.3: A) was carried out in one step and on a non-tilted substrate. Film B was deposited on a tilted 
substrate $\left(\alpha=65^{\circ}\right)$. Deposition C and D were carried out in two steps. Film C was started on a non-tilted substrate which was afterwards tilted to $65^{\circ}$. Film D was started on a $65^{\circ}$ tilted substrate and finished on a non-tilted substrate. All films have a clear [111] out-of-plane alignment as shown in figure VI.3.3. The results of the pole figures of these depositions are summarized in table VI.3.4.

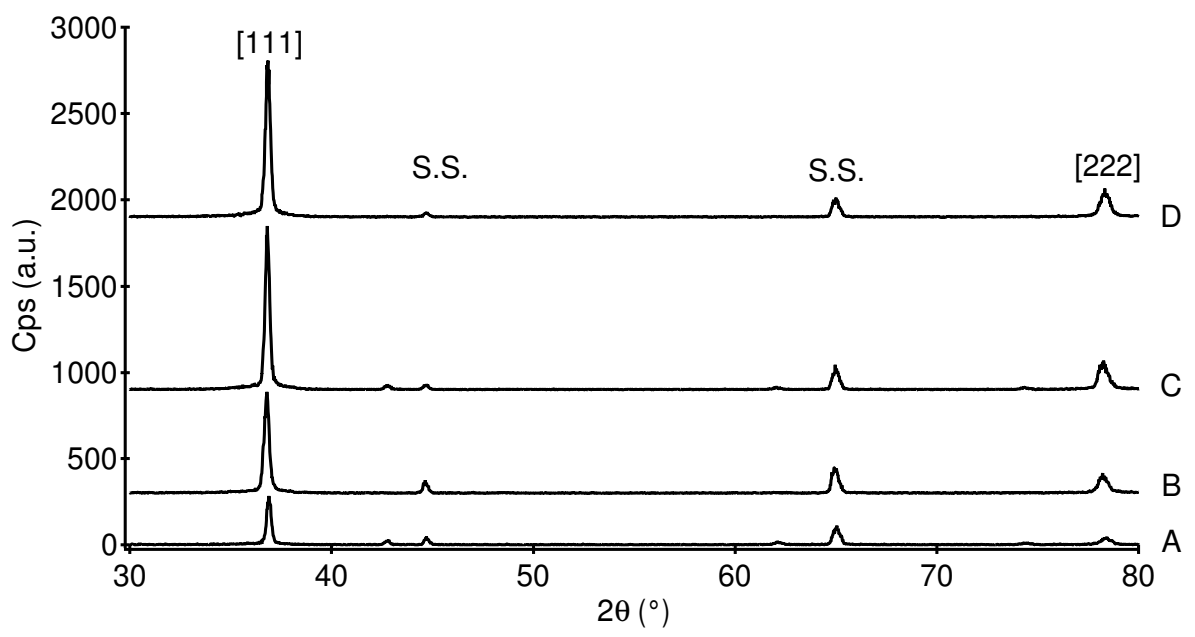

Figure VI.3.3: XRD $\theta / 2 \theta$ results of the four above described depositions. For clarity, the spectra are shifted in the vertical direction.

Table VI.3.4: FWHM and deposition steps of TiN deposition a-d.

\begin{tabular}{|l|l|l|l|}
\hline deposition & $\begin{array}{l}\text { film thickness } \\
(\mu \mathrm{m})\end{array}$ & $\begin{array}{l}\text { Target-substrate } \\
\text { angle } \alpha\end{array}$ & $\begin{array}{l}\text { FWHM in } \\
\text { phi direction }\end{array}$ \\
\hline $\mathcal{A}$ & 1.3 & $0^{\circ}$ & random \\
\hline$B$ & 1.3 & $65^{\circ}$ & $30^{\circ}$ \\
\hline$C$ & 2.6 & $0^{\circ}+65^{\circ}$ & $77^{\circ}$ \\
\hline $\mathcal{D}$ & 2.6 & $65^{\circ}+0^{\circ}$ & $23.5^{\circ}$ \\
\hline
\end{tabular}

As expected, deposition A was random in-plane oriented, while the three others showed an inplane alignment. The degree of in-plane alignment, which is given by the FWHM in the phi direction of the peak in the $\{100\}$ pole figures, changed from $30^{\circ}, 77^{\circ}$ to $23.5^{\circ}$ for deposition $\mathrm{B}, \mathrm{C}$ and $\mathrm{D}$ respectively. The $\{100\}$ pole figures of these four deposition are shown in figure VI.3.5.
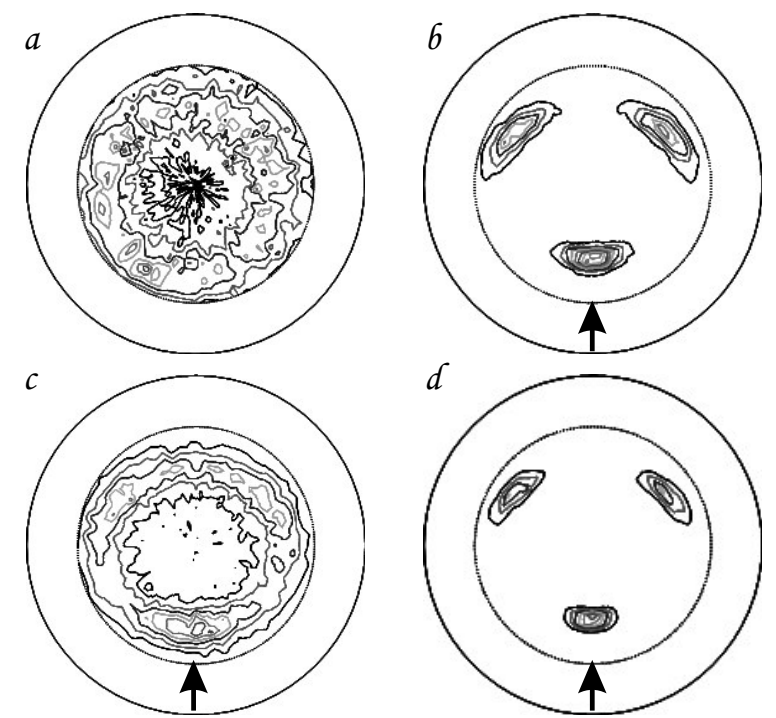

Figure VI.3.5: $\{100\}$ pole figures of TiN films deposited on $+15 \mathrm{~V}$ biased stainless steel, target-substrate distance of $9.5 \mathrm{~cm}, 0,48 \mathrm{~Pa}$ and target-substrate angle of $0^{\circ}(\mathrm{a}), 65^{\circ}(\mathrm{b}), 0^{\circ}+65^{\circ}(\mathrm{c})$ and $65^{\circ}+0^{\circ}(\mathrm{d})$. The arrow indicates the orthogonal projection of the incoming material flux. 
SEM plan views of these four depositions are shown in figure VI.3.6. All four films have faceted column tops. It is also observed that the microstructural in-plane alignment is strongly related to the crystallographic in-plane alignment. This because no microstructural in-plane alignment is observed for deposition $\mathrm{A}$, while it improves for deposition $\mathrm{C}, \mathrm{B}$ and $\mathrm{D}$ respectively.

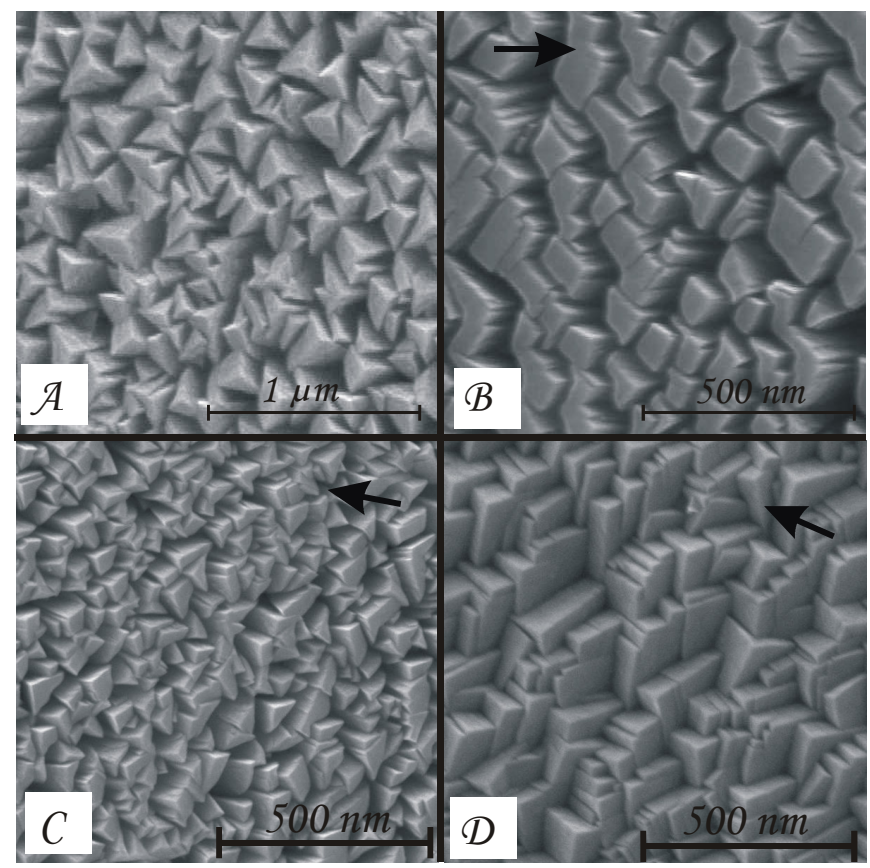

Figure VI.3.6: SEM plan views of TiN films deposited on $+15 \mathrm{~V}$ biased stainless steel, target-substrate distance of $9.5 \mathrm{~cm}, 0,48 \mathrm{~Pa}$ and target-substrate angle of $0^{\circ}(\mathrm{a}), 65^{\circ}(\mathrm{b}), 0^{\circ}+65^{\circ}(\mathrm{c})$ and $65^{\circ}+0^{\circ}(\mathrm{d})$. The arrow indicates the orthogonal projection of the incoming material flux. Clear faceted grains are observed. For deposition B, C and $\mathrm{D}$ also a microstructural in-plane alignment is observed.

TEM cross-section of the depositions $\mathrm{C}$ and $\mathrm{D}$ were taken to investigate if re-nucleation occurred due to the sudden substrate tilt. These TEM cross-sections, together with SAED patterns are shown in figure VI.3.7. It is clearly observed that the columns continuously proceed through the whole film, although the sudden change in tilt direction. From the corresponding SAED patterns, it is also observed that these columns don't change their crystallographic alignment. Hence, it can be concluded that no re-nucleation happens at the interface between the first and the second step of the deposition.
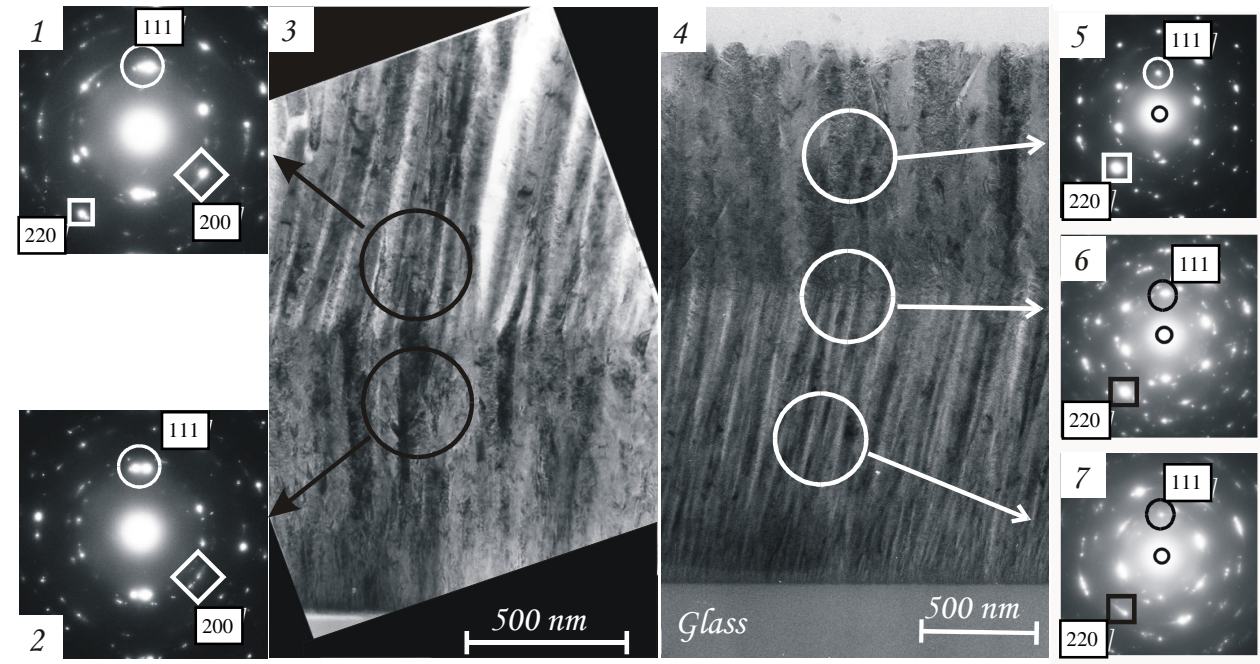

Figure VI.3.7: TEM cross-sections and corresponding SAED patterns for TiN deposition C (1-3) and D (4-7) on glass. The TEM cross-sections show that the columns nicely proceed through the film. The SAED patterns show that the crystallographic alignment of the columns isn't influenced by the sudden change in substrate tilt angle. 
It can be concluded from these observations that the in-plane alignment in $\mathrm{TiN}$ is mainly caused by the $2 \mathrm{D}$ growth. This because the in-plane alignment of deposition $\mathrm{D}$ is much better than the in-plane alignment of deposition $\mathrm{C}$.

- YSZ results:

Again, four depositions were carried out at identical deposition conditions, i.e. a substrate at grounded potential, a target-substrate distance of $13 \mathrm{~cm}$ and a pressure of $0.55 \mathrm{~Pa}$. The sequential steps and the measured FWHM of the poles of $\{111\}$ pole figures of these four depositions are summarized in table VI.3.8.

The first two depositions (see table VI.3.8: A and B) were deposited in one step at a fixed target-substrate angle $\alpha\left(0^{\circ}\right.$ and $55^{\circ}$ respectively). This resulted in films of about $1.6 \mu \mathrm{m}$. The two other depositions (see table VI.3.8: C and D) were deposited in two steps. During the first step a film of $1.6 \mu \mathrm{m}$ was deposited at a fixed angle $\alpha\left(0^{\circ}\right.$ and $55^{\circ}$ respectively), after which the target-substrate angle $\alpha$ was changed in-situ (without stopping the deposition) to another fixed value $\left(55^{\circ}\right.$ and $0^{\circ}$ respectively). In that way, two-stepped films with a total thickness of about $3.2 \mu \mathrm{m}$ were obtained. All films exhibit a clear preferential [002] out-of-plane orientation as was measured by XRD $\theta / 2 \theta$. Pole figures of these YSZ films are shown in figure VI.3.9 and TEM cross-sections in figure VI.3.10. No re-nucleation occurred during the sudden change of the target-substrate angle and the film growth proceeded by local epitaxy. This because it is clearly seen in figure VI.3.10 that the columns continuously proceed the whole film.

Table VI.3.8: FWHM and deposition steps of YSZ deposition a-d.

\begin{tabular}{|l|l|l|l|}
\hline deposition & $\begin{array}{l}\text { film thickness } \\
(\mu \mathrm{m})\end{array}$ & $\begin{array}{l}\text { Target-substrate } \\
\text { angle } \alpha\end{array}$ & $\begin{array}{l}\text { FWHM in } \\
\text { phi direction }\end{array}$ \\
\hline $\mathcal{A}$ & 1.6 & $0^{\circ}$ & random \\
\hline$B$ & 1.6 & $55^{\circ}$ & $21.5^{\circ}$ \\
\hline$C$ & 3.2 & $0^{\circ}+55^{\circ}$ & $50^{\circ}$ \\
\hline $\mathcal{D}$ & 3.2 & $55^{\circ}+0^{\circ}$ & $16.8^{\circ}$ \\
\hline
\end{tabular}
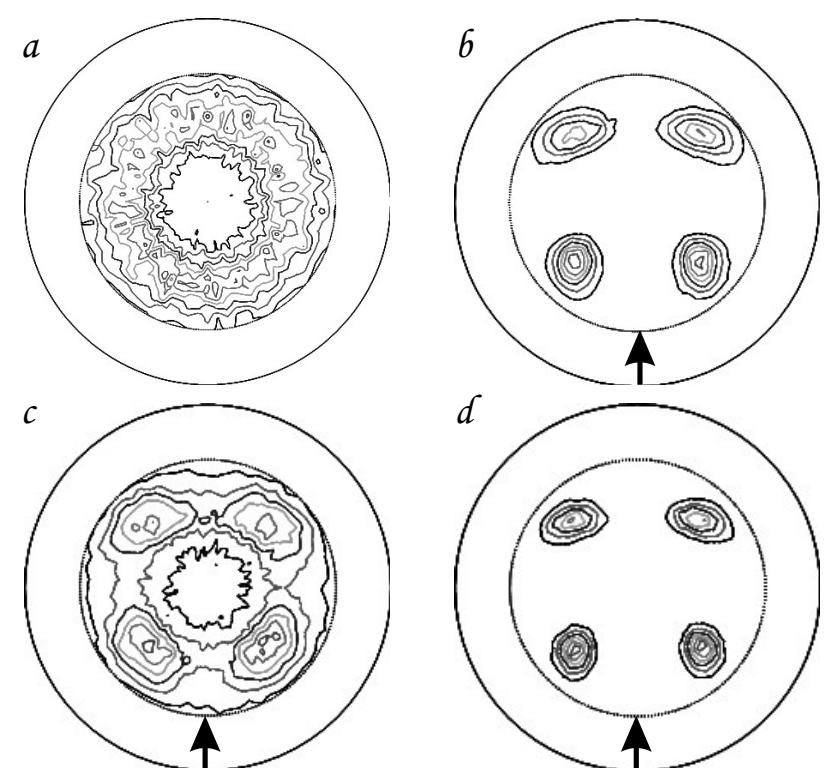

Figure VI.3.9: (111) pole figures of YSZ films deposited on stainless steel at grounded potential, target-substrate distance of $13 \mathrm{~cm}, 0,55 \mathrm{~Pa}$ and target-substrate angle of $0^{\circ}(\mathrm{a}), 55^{\circ}(\mathrm{b}), 0^{\circ}+55^{\circ}$ (c) and $55^{\circ}+0^{\circ}$ (d). The arrow indicates the direction of the incoming material flux. 


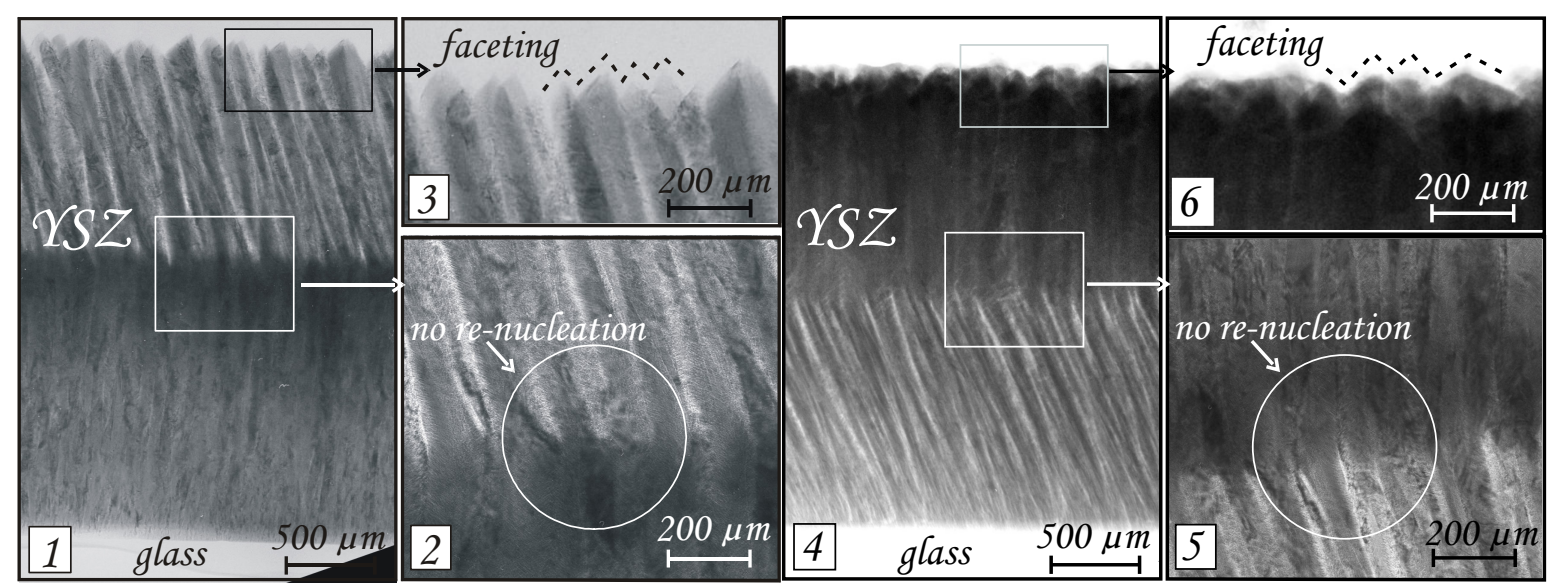

Figure VI.3.10: TEM cross-section images of the depositions C (1 -3) and D (4-6). The figures 1 and 4 are crosssections of the whole film and show clearly the columnar structure, with faceting at the tops. The figures 2 and 5 are enlargements of the middle part of each deposition, clearly demonstrating that no re-nucleation occurs when

the substrate is tilted. The figures 3 and 6 are enlargements of the film surface, indicating the faceting.

It is observed in table VI.3.8 that the degree of the in-plane alignment for deposition $\mathrm{C}(\alpha=$ $\left.0^{\circ}+55^{\circ}\right)$ is much worse than for deposition $\mathrm{B}\left(\alpha=55^{\circ}\right)$, while the degree of in-plane alignment of deposition $\mathrm{D}\left(\alpha=55^{\circ}+0^{\circ}\right)$ is even slightly better compared to deposition $\mathrm{B}$. Again it can be concluded that the $2 \mathrm{D}$ growth is the most important growth mode for the development of the in-plane alignment, because the in-plane alignment in deposition $\mathrm{D}$ is much better than in deposition $\mathrm{C}$.

\section{VI.3.2: In-plane growth rate anisotropy:}

From the above described results, it could be concluded that the resulting in-plane alignment for a deposition carried out in zone $\mathrm{T}$ conditions on a tilted substrate is mainly caused by the $2 \mathrm{D}$ growth mode and only in a minor way by the 3D growth. Therefore, a model for the inplane alignment will first focus on the 2D growth.

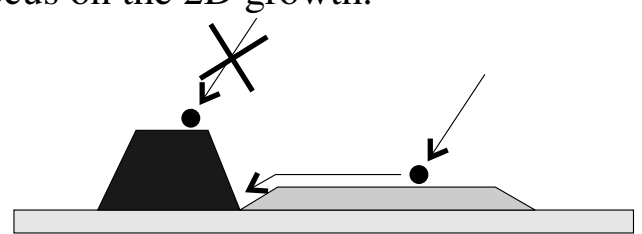

Figure VI.3.11: Schematic drawing of zone T growth as seen in cross-section. Since 2D growth was shown to be the main cause for the in-plane alignment, the contribution of 3D growth was neglected.

As represented in figure VI.3.11 random out-of-plane oriented islands will nucleate. Some of them will offer a high mobility to the incoming adparticles, while the others only offer a small mobility to the adparticles. As discussed in chapter II, this adparticle mobility is related to the number of nearest neighbours offered between the adparticle and the growing plane. Since also the perpendicular growth rate is related to the number of offered nearest neighbours, the grains that offer a high adparticle mobility will have a low perpendicular growth rate and the grains that offer a low adparticle mobility will have a high perpendicular growth rate. As discussed in chapter II, these grains with a high perpendicular growth rate will slowly envelop and overgrow the other out-of-plane oriented grains, and are coloured black in figure VI.3.11. As discussed earlier, these grains are able to expand due to 3D and 2D growth. However, since it was concluded that mainly the 2D growth causes the in-plane alignment, 3D growth will be neglected in a first assumption (as shown in figure VI.3.11).

Due to the relative high mobility on the "wrong" out-of-plane oriented grains, which are represented in light grey, adparticles which initially stroke on such a wrong out-of-plane 
oriented grains will be able to diffuse towards a good out-of-plane oriented grain where they will get incorporated.

Carrying out the deposition on a tilted substrate causes that adparticle diffusion to be directed or biased along the orthogonal projection of the incoming material flux, due to the conservation of the impulse momentum of the adparticles. Such a biased or directed diffusion was first proposed by Abelmann [19] and has been simulated by Kools [20].

Zone $\mathrm{T}$ growth characterised by its clear faceted grains, neglecting the 3D growth, taking into account the biased diffusion and knowing that the grains oriented with the fastest growing direction perpendicular the substrate will overgrow all other grains, results in a growth as schematically represented in figure VI.3.12.

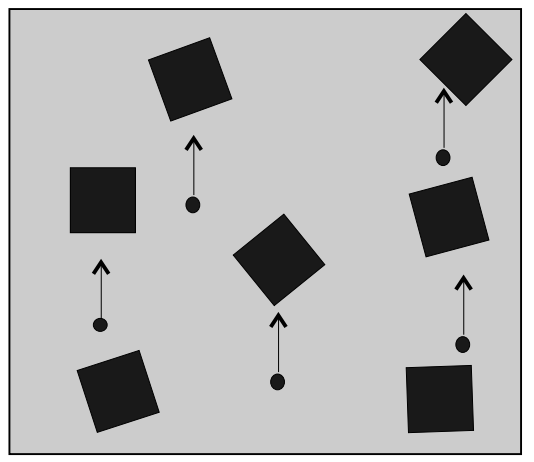

Figure VI.3.12: Schematic drawing of zone T growth as seen in plan view. The directional diffusion of the adparticles is indicated by the arrows. The light grey matrix represents the "wrong" out-of-plane oriented grains, while the dark grains represent the grains oriented with the fastest growing direction perpendicular to the substrate.

As seen in figure VI.3.12, the growth rate of the "good" out-of-plane oriented grains will depend on the amount of diffusing adparticles they capture. Therefore, the capture crosssection or capture length for diffusing adparticles should be calculated as a function of the inplane orientation of the grains. The grains with the largest capture length will have the largest growth rate and hence will be able to overgrow all other grains. Since that capture length will depend on the exact crystal habit of the grains, it has to be calculated for TiN and for YSZ separately.

- In-plane growth rate anisotropy for YSZ:

All depositions of YSZ in zone T conditions were carried out in the situation of $\mathrm{Zr} / \mathrm{Y}$ adatoms and molecular $\mathrm{O}_{2}$ gas. As seen in table V.6.6 this causes the [100] direction to be the geometric fastest growing direction and makes that all grains have a $\{111\}$ faceted crystal habit. This causes the "good" out-of-plane oriented grains to be [100] out-of-plane oriented and to have a square shape as seen in plan view (see figure VI.2.11). The growth rate of these square shaped grains has to be calculated as a function of the in-plane orientation $\omega$, as shown in figure VI.3.13.

As was already discussed in chapter I, the diffusing adparticles must be able to diffuse from one facet to another of a compact grain and thus to cross the corner between two facets during a deposition in zone T conditions. This because otherwise the compact crystal habit is not conserved when continuing the deposition. This corner crossing is also shown in figure VI.3.13 at the upper left.

Assume initially square shaped grains with ' $a$ ' the length of the square side and with an inplane orientation expressed by $\omega$, i.e. the angle between a square side and the orthogonal projection of the incoming material flux. The number of diffusing adparticles that the 
grains catch, depends on the flux of these adparticles and on the capture length of each grain.

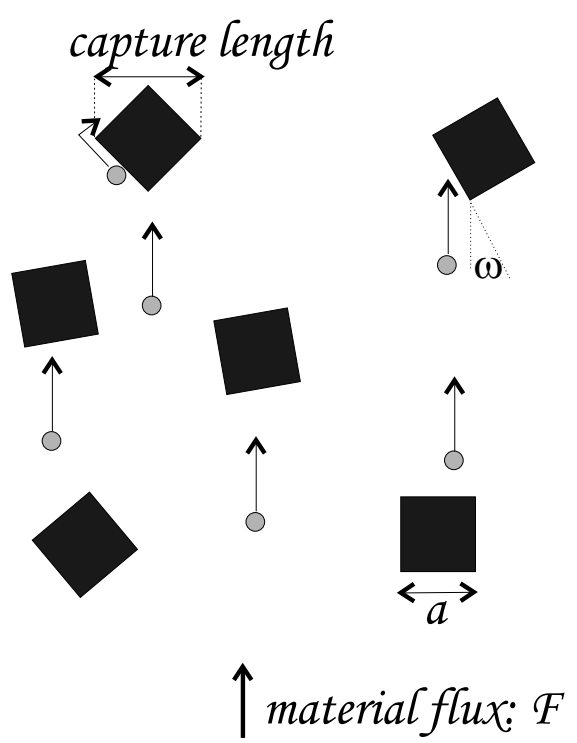

Figure VI.3.13: Schematic drawing of the 2D growth of square shaped [100] out-of-plane oriented grains. $\omega$ stands for the in-plane orientation of the grains. The arrow indicates the direction of the directional diffusion. The corner crossing is indicated at the upper left grain.

If the deposition is carried out on a non tilted substrate $\left(\alpha=0^{\circ}\right)$ there will be no directional diffusion of the adparticles. Since the initial size of the grains is assumed to be $\omega$ independent, there will be no anisotropy in capture length for the diffusing adparticles. As such, no 2D growth rate anisotropy is present and no in-plane alignment is suspected to evolve. This is confirmed by the results of deposition A (see table VI.3.8).

When the deposition is carried out on a tilted substrate, the incoming adparticles will undergo a directional diffusion. In a first approximation, it is assumed that there is no spread on the diffusion direction (i.e. all adparticles will diffuse along one single direction). This directional diffusion leads to a capture length which is $\omega$ dependent and hence to an anisotropy in the $2 \mathrm{D}$ growth rate. The growth can be treated as a stepwise function, in which after each iterative step the size of a grain is given by its area $\left(A_{n}\right)$ and volume $\left(V_{n}\right)$. Taking the corner crossing into account, the area and the volume of each grain in function of the in-plane orientation $\omega$ after each step is given by:

$$
\begin{gathered}
A_{n}(\omega)=A_{n-1}(\omega)+F\left|\cos \left(45^{\circ}-\omega\right)\right| \sqrt{2 A_{n-1}(\omega)} \text { with } A_{0}(\omega)=a^{2} \\
\text { and } V_{n}(\omega)=\left\langle A_{n}(\omega)\right\rangle^{3 / 2} / 3 \sqrt{2} \quad \text { (VI.2) }
\end{gathered}
$$

in which $\mathrm{F}$ is the incoming adparticle flux. Equation VI.1 follows from the dependency of the capture length $C$ on the in-plane orientation $\omega: C=\left|\cos \left(45^{\circ}-\omega\right)\right| \sqrt{2 A_{n-1}(\omega)}$. The volume $V_{n}$ is calculated from the area $A_{n}$ assuming a perfect $\{111\}$ crystal habit, i.e. a pyramid with a square shaped base and the side faces $55^{\circ}$ tilted with respect to the substrate plane.

Figure VI.3.14 shows the relative increase in volume of a grain after step $n$ ( $n=1,10$ and 100 ) as a function of the in-plane orientation $\omega$, for the case that $\mathrm{F} / \mathrm{a}^{2}=0.1$. It is clearly seen that grains with an in-plane orientation of $\omega=45^{\circ}$ have the largest volume increase and thus will overgrow all other grains. These $45^{\circ}$ degrees in-plane oriented grains correspond to the grains with a corner facing towards the orthogonal projection of the material flux. 


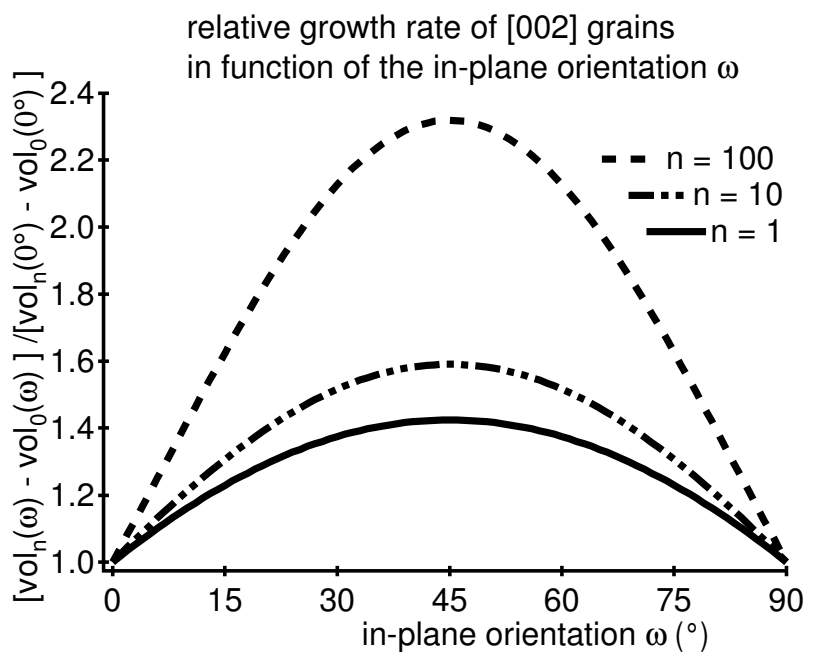

Figure VI.3.14: Relative increase of the volume of an [100] out-of-plane oriented YSZ grain after $n$ steps as a function of the in-plane orientation $\omega$.

Figure VI.3.15 shows the experimentally observed microstructural and crystallographic inplane alignment as seen in the plan view SEM and $\{111\}$ pole figures respectively. Taking into account the $\{111\}$ crystal habit of the grains and the [001] out-of-plane alignment as schematically shown in figure VI.3.15c it is clearly seen that the calculated in-plane alignment corresponds to the experimentally observed in-plane alignment.

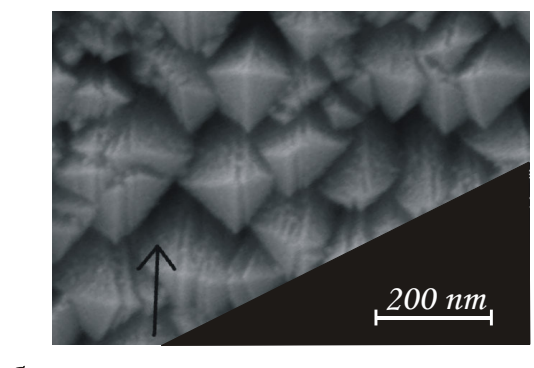

$a$

Figure VI.3.15: Experimentally observed microstructural (a) and crystallographic (b) in-plane alignment for [001] out-of-plane oriented YSZ films deposited in zone T conditions on a tilted substrate. Also the $\{111\}$ faceted crystal habit is shown.

It can be concluded that the resulting in-plane alignment for YSZ depositions carried out in zone $\mathrm{T}$ conditions on a tilted substrate is fully understood as an overgrowth mechanism in which the growth rate anisotropy is caused by the difference in the amount of diffusing adparticles that the [001] out-of-plane oriented grains capture. This difference in captured diffusing adparticles is caused by the growth according to the crystal habit and by the directional or biased diffusion of the adparticles.

- In-plane growth rate anisotropy for TiN:

Analogous calculations of the anisotropic in-plane growth rate must be carried out for TiN. It was discussed in chapter $\mathrm{V}$, that all $\mathrm{TiN}$ depositions carried out in zone $\mathrm{T}$ conditions were done in the situation of Ti adatoms and molecular $\mathrm{N}_{2}$ gas, as could be measured by mass spectroscopy. This means that the TiN grains are all $\{100\}$ faceted and that the [111] orientation is the final out-of-plane alignment. As shown in figure VI.2.5, this means that all "good" out-of-plane oriented grains are triangular shaped as seen in plan view. Again, the number of diffusing adparticles that these triangular shaped grains capture as a function of their in-plane orientation must be calculated. 
Assume initially triangular shaped grains with ' $a$ ' the length of the side and with an inplane orientation expressed by $\omega$, i.e. the angle between a side and the orthogonal projection of the incoming material flux, as shown in figure VI.3.16. Again, it is assumed that all adparticles diffuse along one single direction, i.e. along the orthogonal projection of the incoming material flux.

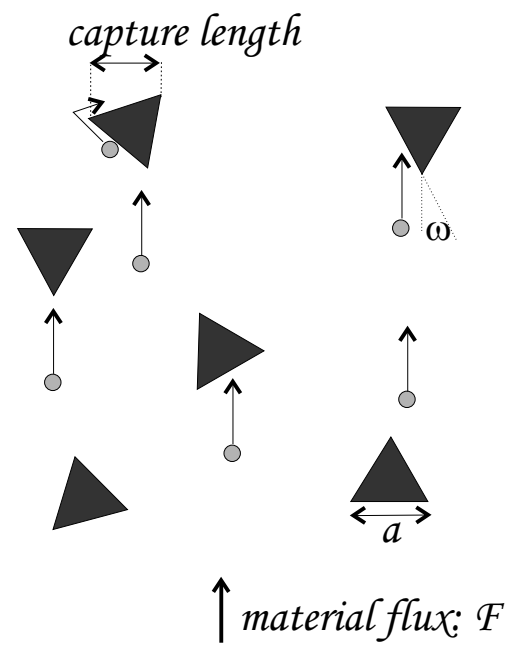

Figure VI.3.16: Schematic drawing of the 2D growth of triangular shaped [111] out-of-plane oriented grains. $\omega$ stands for the in-plane orientation of the grains. The arrow indicates the direction of the directional diffusion. The corner crossing is indicated at the upper left grain.

Also in this case, the increase in area and volume of the [111] out-of-plane oriented grains can be calculated, taking into account the cross-corner effect of the diffusing adparticles and the growth according to the $\{100\}$ faceted crystal habit:

$$
\begin{gathered}
A_{n}(\omega)=A_{n-1}(\omega)+F\left|\cos \left(\omega-30^{\circ}\right)\right| \sqrt{4 A_{n-1}(\omega) / \sqrt{3}} \text { with } A_{0}(\omega)=\frac{\sqrt{3} a^{2}}{4} \\
\text { and } V_{n}(\omega)=0.416\left\langle A_{n}(\omega)\right\rangle^{3 / 2}(\mathrm{VI} .4)
\end{gathered}
$$

$\mathrm{F}$ indicates the flux of adparticles and $\mathrm{n}$ the growth step.

Calculation of the volume increase for several $n$ values shows that there are two in-plane alignments which both have the largest growth rate, i.e. $\omega=30^{\circ}$ and $90^{\circ}$ as shown in figure VI.3.17. These two in-plane orientations correspond to a triangular shaped grain with a top or a side towards the diffusion direction respectively.

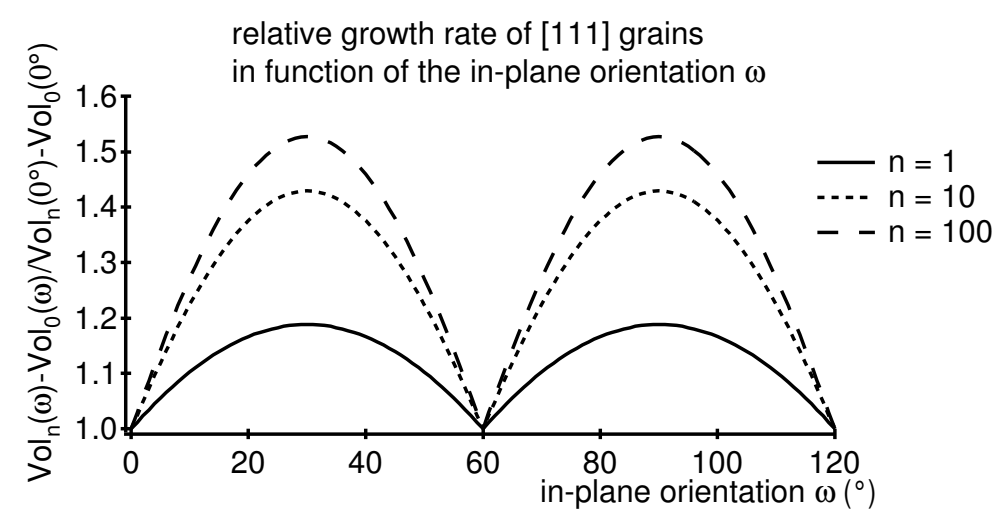

Figure VI.3.17: Relative increase of the volume of a [111] out-of-plane oriented TiN grain after $\mathrm{n}$ steps as a function of the in-plane orientation $\omega$. Two in-plane alignments have the fastest volume increase.

From the experimental observations by SEM plan view and XRD pole figures (see figure VI.3.18) it is clearly observed that the grains with an in-plane alignment of $\omega=90^{\circ}$ overgrow all other in-plane aligned grains. 

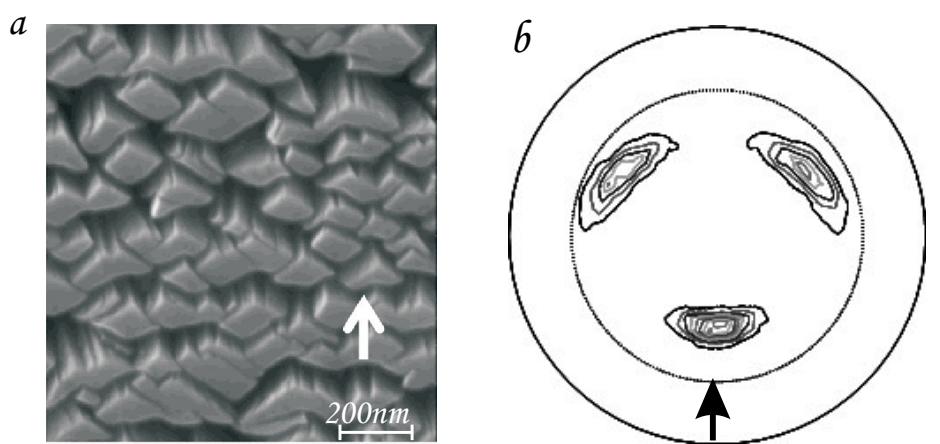

Figure VI.3.18: Experimental observed microstructural and crystallographic in-plane alignment of TiN thin films deposited at zone T conditions on a tilted substrate by SEM (a) and XRD $\{100\}$ pole figure (b). The arrow indicates the orthogonal projection of the material flux, and thus the diffusion direction of the adparticles.

However, no firm reason why the grains with an in-plane alignment of $90^{\circ}$ overgrow the grains with an in-plane alignment of $30^{\circ}$ can be given up to now. Only some possible suggestions are mentioned in the appendix.

\section{VI.3.3: Considerations on the two-stepped depositions B and D (see VI.3.1):}

From the above proposed mechanism for the in-plane alignment, it can be concluded that the resulting in-plane alignment for a deposition carried out in zone $\mathrm{T}$ conditions on a tilted substrate is understood as an overgrowth mechanism in which the grains with the largest growth rate overgrow the other grains. This in-plane growth rate anisotropy is caused by

- the directional or biased diffusion of the adparticles due to the conservation of their momentum when hitting a tilted substrate

- the anisotropy of the capture length for diffusing adparticles as a function of the in-plane orientation $\omega$ due to the growth of the grains according to their kinetically determined faceted crystal habit.

Due to the anisotropy in volume increase, the fastest growing grains overgrow the other grains. These fastest growing grains, will also be the tallest. This means that in case of shadowing, these grains will be the least shadowed and thus will be favoured to overgrow the other grains. This also explains why the deposition D, i.e. the two-stepped deposition started on a tilted substrate and then continued on a non-tilted substrate results in a better in-plane alignment than the one-step deposition B (see table VI.3.4 and VI.3.8).

After the first step of deposition D, the same in-plane alignment as in deposition B developed, but then can further improve when continuing the deposition on a non-tilted substrate just by shadowing since the grains with the good in-plane orientation are the tallest.

\section{4: Models in literature for the in-plane alignment}

Beside the above proposed model for the development of an in-plane alignment in a thin film deposited on a tilted substrate, two other models were described in literature. These two models will be discussed in this section. A first model was presented by K. Hasegawa et al. and the second was presented by O.P. Karpenko et al.

\section{- Model of K. Hasegawa:}

$\mathrm{K}$. Hasegawa et al. deposited biaxially aligned $\mathrm{YSZ}$ and $\mathrm{MgO}$ on a tilted substrate by Pulsed Laser Deposition and e-beam evaporation respectively $[12,13]$. A schematic drawing of their observed in-plane alignment is given in figure VI.4.1. They observed a [100] out-of-plane 
oriented YSZ film and also observed peaks $45^{\circ}$ rotated in the phi direction with respect to the orthogonal projection of the material flux when taking a $\{111\}$ pole figure. Hence, they observed the same biaxial alignment as described for our YSZ depositions. In case of $\mathrm{MgO}$, Hasegawa et al. observed a [111] out-of-plane alignment and an in-plane alignment with a (100) plane towards the incoming material flux. This corresponds to our observations for TiN, what seems logic since both materials have the same rocksalt structure.
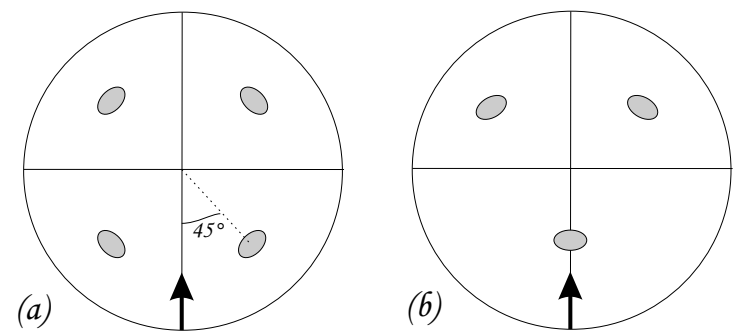

Figure VI.4.1: Schematic drawing of a typical $\{111\}$ pole figure of YSZ (a) and a typical $\{100\}$ pole figure of $\mathrm{MgO}$ (b) for the observations of Hasegawa et al. The orthogonal projection of the incoming material flux is indicated by the arrow.

SEM cross-sections of the YSZ films indicated an evolutionary overgrowth resulting in a columnar structure in which the column tops are clearly faceted [12]. SEM plan views of the $\mathrm{MgO}$ films indicated also faceted column tops with a triangular shape a seen in plane view. Hence also the observed microstructure compared to our observations.

Hasegawa proposed a first model to explain the in-plane alignment of $\mathrm{MgO}$ [13]. In a next publication [21], that model was adapted giving rise to a second model which was used to explain the out-of-plane and in-plane alignment of $\mathrm{MgO}$ and YSZ.

It was argued that the grains with the largest growth rate would overgrow all other grains. Hence, if the growth rate depends on the in-plane orientation of the grains, an in-plane alignment will develop. In order to calculate the growth rate of a grain, they start from the assumption that the growth rate $\mathrm{R}$ of a plane is in proportion to the vapour flux $\mathrm{J}$ per unit area of the growth face. The growth of a grain taking into account this assumption is schematically represented in figure VI.4.2.

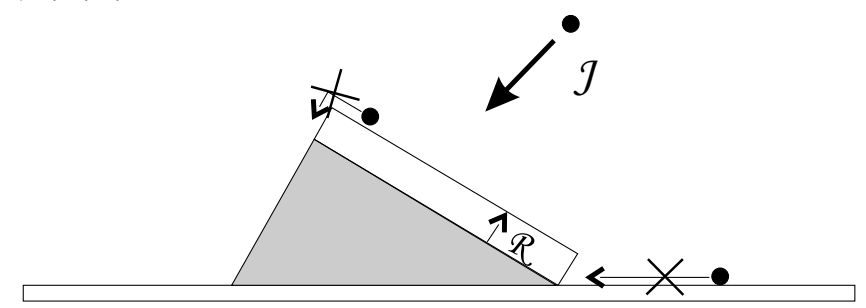

Figure VI.4.2: Schematic drawing of the growth of a grain assuming that the growth rate of a plane is in proportion to the amount of the vapour flux J per unit area of the growth face. Also the prohibition of the 2D growth and the diffusion from one facet to another facet is shown.

However, this assumption implies two basic differences from the model proposed in section VI.3.

-First of all, it neglects the 2D growth and implies that the grains only grow by 3D growth, since it was assumed that the growth rate of a plane depends on the amount of adparticles arriving directly from the vapour flux. This is also shown in figure VI.4.2.

-Secondly it implies that the adparticles can't diffuse from one facet to another facet of a grain, since it was assumed that the growth rate of a plane or facet is in proportion to the amount of adparticles captured by that plane and not by another plane. This is also shown in figure VI.4.2. 
In case of $\mathrm{MgO}$ this growth face was a (100) facet, since they observed that the grains are $\{100\}$ faceted. However, in case of YSZ they assumed a $\{110\}$ faceting of the grains, following the reasoning of McIntyre et al. [22].

In section VI.3. it was shown that the 2D growth can not be neglected since it is the main driving force for the resulting in-plane alignment. In chapter I and II, it was also clearly described that the diffusion of the adparticles from one plane or facet to another facet of the same grain must be allowed. This because the grains otherwise can't grow according to their kinetically determined crystal habit.

Moreover, it was also discussed that the YSZ grains are $\{111\}$ faceted instead of $\{110\}$ faceted.

It can be concluded that the model presented by Hasegawa et al. is incorrect since it makes some assumptions which are physically not allowed.

\section{- Model of O.P. Karpenko:}

Karpenko et al. deposited biaxially aligned Mo by sputtering on an inclined or moving substrate [5]. They proposed a model for the development of the in-plane alignment based on atomic self-shadowing and on an anisotropic crystallographic growth rate. According to their calculations, the atomic self-shadowing caused an anisotropy in the geometric growth rate. Finally they argued that the grains oriented in such a way that the crystallographic fastest growing direction corresponds to the geometric fastest growing direction will have the largest growth rate and thus will overgrow all other grains, causing the in-plane alignment [23].

Again, there are some drawbacks to this model.

- The anisotropy in geometric growth rate was calculated assuming an atomic self-shadowing effect as shown in figure VI.4.3.
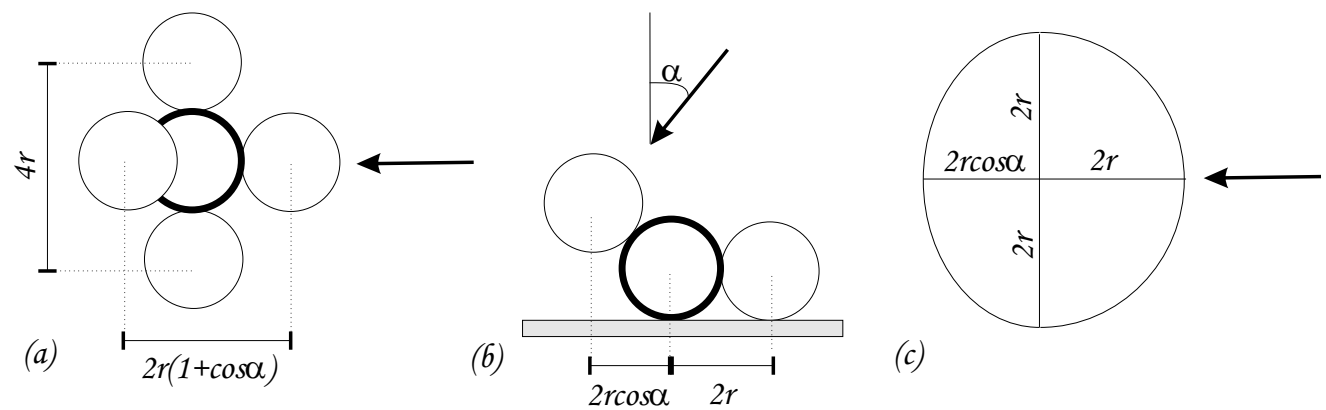

Figure VI.4.3: Schematic drawing of the influence of atomic shadowing on the capture length and capture area around an atom during the deposition on a tilted substrate. The capture lengths as seen in plan view (a), as seen

in cross-section (b) and a sketch of the total capture area (c). $\mathrm{r}$ is the atomic radius. The arrow indicates the orthogonal projection, or the direction itself, of the incoming material flux.

It was assumed that an adparticle coming from the gas phase will get attached to another chemisorbed atom as it comes close enough to that atom. The maximum distance at which an adparticle can still attach to an atom on the surface is called the capture length. That capture length along the direction perpendicular to the orthogonal projection of the incoming material flux is defined as $2 \mathrm{r}$ in which $\mathrm{r}$ is the radius of an atom. This is clearly seen in figure VI.4.3a. However, as seen in cross-section view (figure VI.4.3b) the capture length parallel to the orthogonal projection of the incoming material flux decreases when the flux is nonperpendicular. As seen from figure VI.4.3b, that capture length is now $2 r(1+\cos \alpha)$ in which $\alpha$ is the tilt angle of the substrate with respect to the incoming material flux. As seen in figure VI.4.3c, this makes that the capture area is more or less an ellipse with major axis $4 \mathrm{r}$ and minor axis $2 \mathrm{r}(1+\cos \alpha)$. 
Hence, Karpenko concluded that the direction perpendicular to the orthogonal projection of the material flux has the largest geometric growth rate, while the direction parallel to the orthogonal projection of the material flux has the lowest geometric growth rate.

However, assuming that the growth proceeds as described by Karpenko implies again that the growth only proceeds by 3D growth and also that the diffusion of adparticles on a grain is not allowed. As mentioned earlier, it was experimentally proved that the $2 \mathrm{D}$ growth is the main cause for the in-plane alignment and it was also argued that adparticle diffusion on a grain must be allowed in order to achieve clear faceted grains. Hence, the model proposed by Karpenko et al. to calculate the anisotropy in geometric growth rate is incorrect.

- Karpenko et al. mentioned that the growth rate of a crystallographic plane depends on many parameters [23] and can differ from one plane to another plane, but proposed no model to explain which crystallographic plane should have the largest growth rate. They only characterised the shape of the grains by SEM plan-view and noticed that the grains were elongated along the [100] orientation, after which they assumed that the [100] direction is the fastest growing crystallographic direction. Since the resulting in-plane alignment corresponds to the grains aligned with the fastest growing crystallographic direction along the fastest growing geometric orientation, i.e. perpendicular to the orthogonal projection of the material flux, this means that they explained the observed in-plane alignment with their own experimental observations...

It can be concluded that both the model of Hasegawa and the model of Karpenko relies on some assumptions which are physically incorrect.

\section{5: Conclusions}

In section VI.2 it was described that all biaxially aligned thin films deposited by our deposition system were deposited in zone $\mathrm{T}$ conditions and on a tilted substrate. Carrying out the depositions in zone $\mathrm{T}$ conditions allow the grains to grow by $3 \mathrm{D}$ growth, i.e. the direct capture of adparticles from the gas phase, and by 2D grow, i.e. incorporating adparticles which first stroke on the bare substrate or on different out-of-plane oriented grains and then diffused towards the expanding grain.

By carrying out depositions in two steps, it could be shown that the 2D growth is the main cause for the resulting in-plane alignment, what allowed to neglect the $3 \mathrm{D}$ growth in a first assumption.

The growth in zone $\mathrm{T}$ conditions also causes the grains to grow according their kinetically determined faceted crystal habit. This faceting of the grains, together with a directional diffusion of the adparticles due to the conservation of the momentum, resulted in a capture length for these diffusing adparticles which depends on the in-plane orientation of the grains. It was argued that the grains with the largest capture length have the largest volume increase and thus are able to overgrow all other oriented grain, what causes the in-plane alignment. Finally, the growth rate of the "good" out-of-plane oriented grains has been calculated as a function of the in-plane orientation for YSZ and TiN grains. It could be shown that the resulting in-plane alignment of YSZ is fully understood, while there is still no explanation why the TiN grains with an in-plane orientation of $90^{\circ}$ will overgrow the TiN grains with an in-plane orientation of $30^{\circ}$. 


\section{References}

1: G. De Winter, S. Mahieu, I. De Roeck, R. De Gryse, J. Denul: IEEE T. Appl. Supercond. 13(2) (2003) p.2567-2570

2: P. Ghekiere, S. Mahieu, G. De Winter, R. De Gryse, D. Depla: J. Cryst. Growth 271 (2004) p.462-468

3: P. Ghekiere, S. Mahieu, D. Depla, R. De Gryse, O.I. Lebedev and G. Van Tendeloo: presentation at "Reactive sputter deposition" 8-9/12/2005 Delft

4: P. Ghekiere: PH.D. thesis: to be published

5: O.P. Karpenko, J.C. Billello, S.M.Yalisove: J. Appl. Phys. 76(8) (1994) p.4610-4617

6: M. Bauer, J. Schwachulla, S. furtner, P. Berberich, H. Kinder: Inst. Phys. Conf. 158 (1997) p. $1077-1080$

7: A. Mancini, G. Celentano, F. Fabbri, V.Galluzzi, P. Petrisor, A. Rufoloni, E. Varesi, A. Vannozzi: Int. J. Mod. Phys. B 17(4-6) (2003) p.886-892

8: G.C.A.M. Janssen, P.F.A. Alkemade, V.G.M. Sivel, S. Y. Grachev, J.D. Kamminga: J. Vac. Sci. Technol. A 22(4) (2004) p.1773-1777

9: T. Hashimoto, K. Okamoto, K. Hara, M. Kamiya, H. Fujiwara: Thin Solid Films 182 (1989) p.197-207

10: A. Rodriguez-Navarro, W. Otano-Rivera, J.M. Garcia-Ruiz, R. Messier, L.J. Pilione : J. Mater. Res. 12(7) (1997) p.1689-1692

11: K. Itoh, F. Ichikawa, Y. Ishida, K. Okamoto, T. Uchiyama, I. Iguchi: J. Magn. Mag. Mat. 248 (2002) p.112-120

12: K. Hasegawa, K. Fujino, H. Mukai, M. Konishi, K. Hayashi, K. Sato, S. Honjo, Y. Sato, H. Ishii, Y. Iwata: Appl. Supercond. 4(10-11) (1996) p.487-493

13: K. Hasegawa, N. Hobara, Y. Nakamura, T. Izumi, Y. Shiohara: Phys. C 354 (2001) p.424428

14: W.A.J. Quinton, F. Baudenbacher : Phys. C 292 (1997) p.243-247

15: M.P. Chudzik, R.A. Erck, Z.P. Luo, D. J. Miller, U. Balachandran, C.R. Kannewurf: Phys. C 341-348 (2000) p.2483-2484

16: B. Ma, M. Li, B.L. Fisher, R.E. Koritala, U. Balachandran: Phys. C 382 (2002) p.38-42

17: R.E. Koritala, M.P. Chudzik, Z. Luo, D.J. Miller, C.R. Kannewurf, U. Balachandran: IEEE T. Appl. Supercond. 11(1) (2001) p.3473-3476

18: Y. Sato, K. Matsuo, Y. Takahashi, K. Muranaka, K. Fujino, S. Hahakura, K. Ohmatsu, H. Takei: IEEE T. Appl. Supercond. 11(1) (2001) p.3365-3370

19: L. Abelmann: Thin Solid Films 305 (1997) p.1-21

20: J.C.S. Kools: J. Vac. Sci. Technol. A23(1) (2005) p.85-89

21: K. Hasegawa, Y. Nakamura, T. Izumi, Y. Shiohara: Phys. C 378-381 (2002) p.955-959

22: P.C. McIntyre, K.G. Ressler, N. Sonnenberg, M.J. Cima: J. Vac. Sci. Technol. A 14 (1996) p.210-215

23: O.P. Karpenko, J.C. Bilello, S.M. Yalisove: J. Appl. Phys. 82(3) (1997) p.1397-1403 


\section{Appendix:}

It was mentioned that no explanation could be given why the TiN grains with an in-plane orientation of $90^{\circ}$ will overgrow the grains with an in-plane orientation of $30^{\circ}$. Here, a possible explanation is suggested.

Up to now, it was tacitly assumed that a diffusing adparticle which finally reaches a "good" out-of-plane oriented grain will also take part to the growth of this grain. This means that the sticking coefficient, i.e. the fraction of adparticles which really gets incorporated in the growing unit, is assumed to be unity on such a "good" out-of-plane oriented grain. Of course, this will not always be true since some adparticles reaching a "good" out-of-plane oriented grains will eventually desorb again from this grain. The larger this desorption rate the lower the growth rate of these grains. Hence, if the desorption rate depends on the in-plane orientation, it can influence the in-plane alignment.

As shown in figure A, the arriving flux density per facet is larger for a triangular shaped grain with its side towards the flux than for a grain with its top towards the flux. By pure geometric considerations, it is seen that this flux density is 2 times larger.

(a)

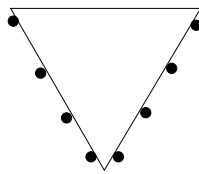

(6)

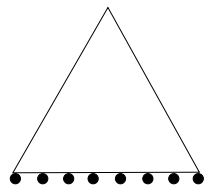

\section{$\uparrow_{\text {material flux }}$}

Figure A: The arriving flux density per facet facing towards the material flux for a grain with an in-plane orientation of $30^{\circ}$ (a) and $90^{\circ}$ (b).

As already discussed in chapter I, the larger the flux density, the higher the probability that the diffusing adparticles will encounter another adparticle and can form a dimer. This means that also the sticking coefficient or the probability the get incorporated in the grain increases with increasing flux density since dimers are more stable than monomers and thus desorb less fast. As such, the grain with the $90^{\circ}$ in-plane orientation could have a larger growth rate compared to the grain with an in-plane orientation of $30^{\circ}$ due to the higher flux density and thus the lower desorption rate.

It should be remarked that the upper mechanism is only a suggestion and no definite explanation why the grains with an in-plane orientation of $90^{\circ}$ overgrow the grains with an in-plane orientation of $30^{\circ}$. 


\section{Chapter VII: Degree of in-plane alignment.}

\section{1: Introduction}

The development of the out-of-plane alignment has been described in chapter $\mathrm{V}$ and the inplane alignment in chapter VI. Aside from the development of this out-of-plane and in-plane alignment, it was also observed that the degree of alignment was strongly influenced by a lot of deposition parameters. Investigating the influence of each parameter separately was often not straightforward, because the degree of alignment seemed not only to depend on common parameters like pressure, target-substrate distance, target-substrate angle $\alpha$, film thickness, substrate bias,... but also on more unexpected parameters like target usage, condition of the chamber walls,...

In this chapter, it is attempted to give an overview of the influence of all these parameters on the degree of in-plane alignment. Finally, it will be argued that all these external parameters can be reduced to two fundamental parameters, i.e. the mobility during the growth and the angular spread of the incoming material flux.

\section{2: Experimental observations}

First, an overview of the observed influence of most deposition parameters on the degree of in-plane alignment is given. That degree of in-plane alignment was measured by the full width at half maximum (FWHM) of a diffraction pole in a pole figure along the phi-direction. In case of TiN, all films are [111] out-of-plane aligned and the degree of in-plane alignment has been determined from $\{200\}$ pole figures, while in case of YSZ the films were [100] out-ofplane aligned and $\{111\}$ pole figures are used to measure the in-plane alignment. The influence of some parameters has been investigated for TiN as well as for YSZ and will be reported simultaneously, while other parameters were only investigated for YSZ.

- Influence of the substrate bias $\mathrm{V}_{\underline{s}}$ :

TiN thin films of $\sim 1.05 \mu \mathrm{m}$ were deposited by a $1 / 9$ unbalanced magnetron (see chapter III) on polycrystalline stainless steel, at a pressure of $0.58 \mathrm{~Pa}$, a target-substrate distance of $13 \mathrm{~cm}$, an $\mathrm{Ar} / \mathrm{N}_{2}$ ratio of 55/5 and a target-substrate angle $\alpha$ of $55^{\circ}$. The substrate bias has been varied between -30 and $+30 \mathrm{~V}$ respective to the ground potential.

YSZ thin films of $\sim 1.6 \mu \mathrm{m}$ were deposited by a $1 / 9$ unbalanced magnetron on polycrystalline stainless steel, at a pressure of $0.55 \mathrm{~Pa}$, a target-substrate distance of 13 $\mathrm{cm}$, an $\mathrm{Ar} / \mathrm{O}_{2}$ ratio of $58 / 2.5$ and a target-substrate angle $\alpha$ of $55^{\circ}$. The substrate bias was again varied between -30 and $+30 \mathrm{~V}$ respective to the ground potential.
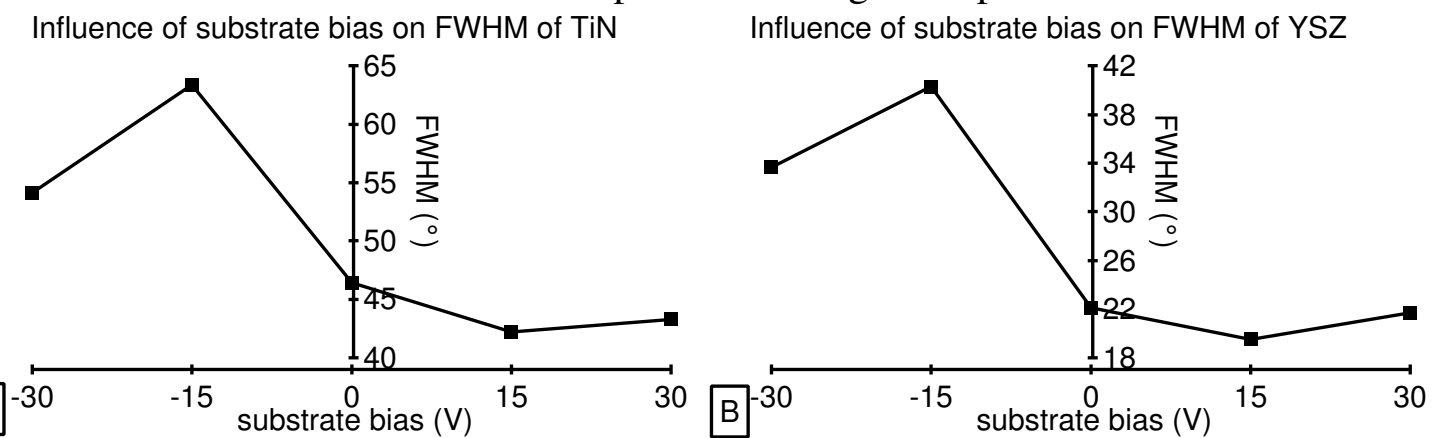

Figure VII.2.1: Influence of the substrate bias on the degree of in-plane alignment for TiN (a) and YSZ (b). For both materials, an optimum around $+15 \mathrm{~V}$ is observed.

For both materials, a strong increase of the FWHM, and thus a worsening of the in-plane alignment is observed when applying a negative bias. 
- Influence of the target-substrate angle $\alpha$ :

TiN thin films of $\sim 0.7 \mu \mathrm{m}$ were deposited by a $1 / 9$ unbalanced magnetron on grounded polycrystalline stainless steel $\left(\mathrm{V}_{\mathrm{s}}=0 \mathrm{~V}\right)$, at a pressure of $0.58 \mathrm{~Pa}$, a target-substrate distance of $13 \mathrm{~cm}$ and an $\mathrm{Ar} / \mathrm{N}_{2}$ ratio of 55/5. The target-substrate angle $\alpha$ has been varied between $30^{\circ}$ and $60^{\circ}$. Remember that the target-substrate angle is defined as the angle between the substrate normal and the target normal.

YSZ thin films of $\sim 1.3 \mu \mathrm{m}$ were deposited by a $1 / 9$ unbalanced magnetron on a positively biased polycrystalline stainless steel substrate $\left(\mathrm{V}_{\mathrm{s}}=+15 \mathrm{~V}\right)$, at a pressure of $0.425 \mathrm{~Pa}$, a target-substrate distance of $13 \mathrm{~cm}$ and an $\mathrm{Ar} / \mathrm{O}_{2}$ ratio of 60/2.5. The target-substrate angle $\alpha$ was varied between $30^{\circ}$ and $55^{\circ}$.

The FWHM of the poles in the phi-direction are shown in figure VII.2.2.
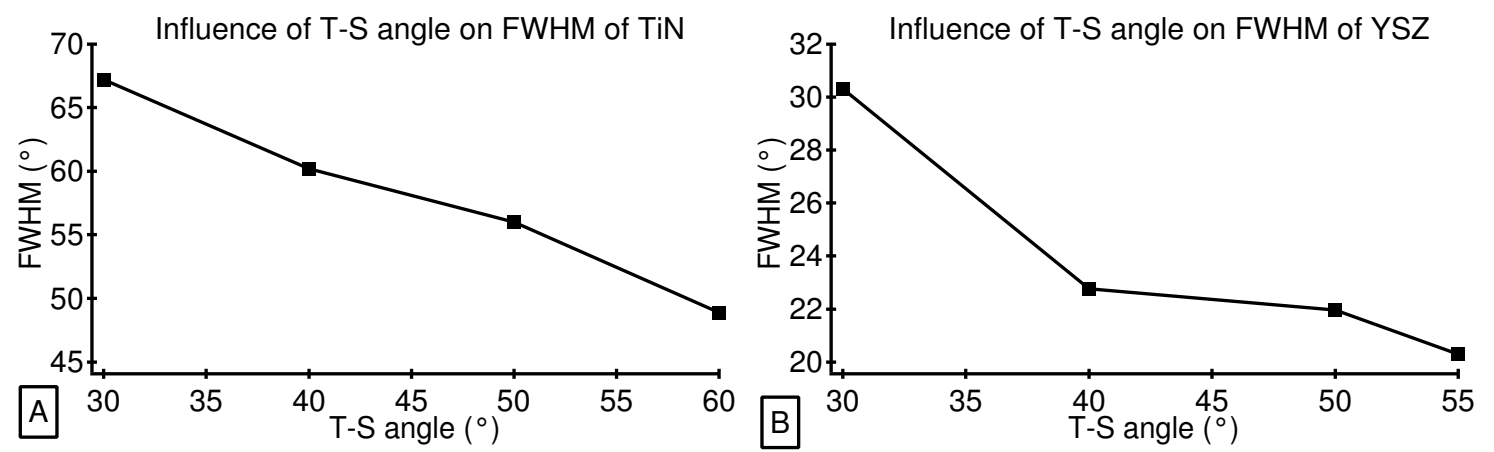

Figure VII.2.2: Influence of the target-substrate angle $\alpha$ on the degree of in-plane alignment for TiN (a) and YSZ (b). For both materials, an improving in-plane alignment with increasing angle $\alpha$ is observed.

The degree of in-plane alignment improves with increasing target-substrate angle $\alpha$, as well as for TiN as for YSZ.

- Influence of the pressure P:

TiN thin films of $\sim 1 \mu \mathrm{m}$ were deposited by a 1/9 unbalanced magnetron on grounded polycrystalline stainless steel $\left(\mathrm{V}_{\mathrm{s}}=0 \mathrm{~V}\right)$, at a target-substrate distance of $13 \mathrm{~cm}$, a targetsubstrate angle of $55^{\circ}$ and an $\mathrm{Ar} / \mathrm{N}_{2}$ ratio of 55/5. The pressure has been varied between 0.38 and $0.68 \mathrm{~Pa}$, by adapting the pumping speed.

YSZ thin films of $\sim 1.3 \mu \mathrm{m}$ were deposited by a $1 / 9$ unbalanced magnetron on a positively biased polycrystalline stainless steel $\left(\mathrm{V}_{\mathrm{s}}=+15 \mathrm{~V}\right)$, at a target-substrate distance of $13 \mathrm{~cm}$, a target-substrate angle of $55^{\circ}$ and an $\mathrm{Ar} / \mathrm{O}_{2}$ ratio of $60 / 2.5$. The pressure was varied between 0.2 and $0.8 \mathrm{~Pa}$, again by adapting the pumping speed.

The FWHM of the poles in the phi-direction are shown in figure VII.2.3.
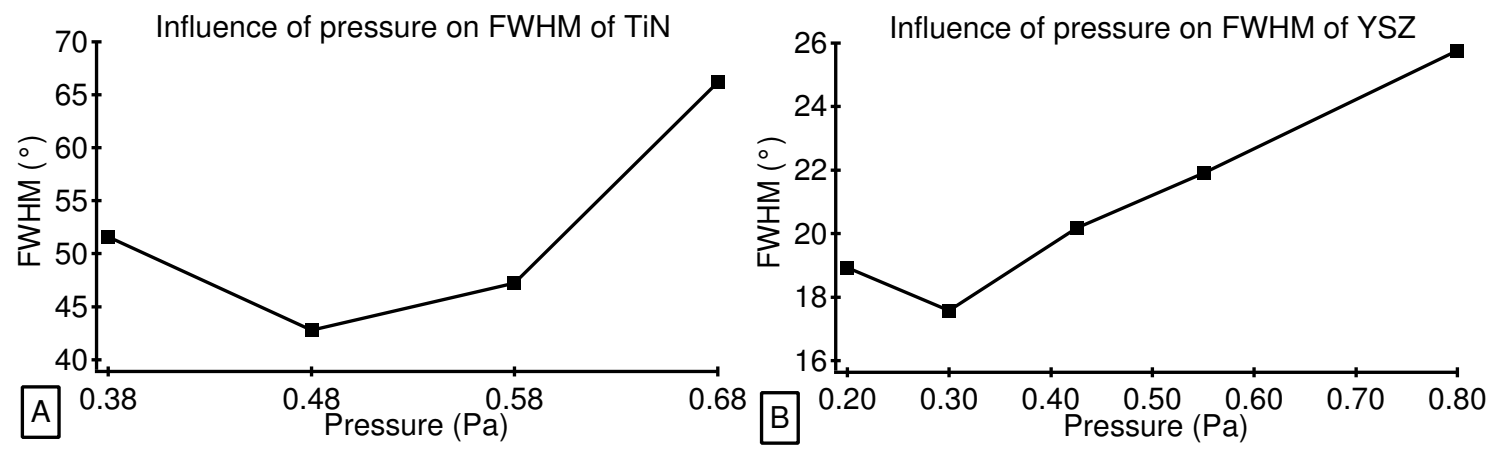

Figure VII.2.3: Influence of the pressure on the degree of in-plane alignment for TiN (a) and YSZ (b). For both materials, an improving in-plane alignment with decreasing pressure is observed, up to an optimum. At too low pressures, the in-plane alignment again get worse. 
For both materials, it is observed that the in-plane alignment first improves with decreasing the pressure, up to an optimum. In case of TiN this optimum is $0.48 \mathrm{~Pa}$, while it is $0.3 \mathrm{~Pa}$ in case of YSZ. At too low pressures, the in-plane alignment get worse again.

- Influence of the target-substrate distance d(T-S):

TiN thin films of $\sim 1.25 \mu \mathrm{m}$ were deposited by a $1 / 9$ unbalanced magnetron on grounded polycrystalline stainless steel $\left(\mathrm{V}_{\mathrm{s}}=0 \mathrm{~V}\right)$, at a target-substrate angle of $55^{\circ}$, a pressure of $0.58 \mathrm{~Pa}$ and an $\mathrm{Ar} / \mathrm{N}_{2}$ ratio of 55/5. The target-substrate distance has been varied between 9 and $15 \mathrm{~cm}$.

YSZ thin films of $\sim 1.3 \mu \mathrm{m}$ were deposited by a $1 / 9$ unbalanced magnetron on a positively biased polycrystalline stainless steel $\left(\mathrm{V}_{\mathrm{s}}=+15 \mathrm{~V}\right)$, at a target-substrate angle of $55^{\circ}$, a pressure of $0.3 \mathrm{~Pa}$ and an $\mathrm{Ar} / \mathrm{O}_{2}$ ratio of $60 / 2.5$. The target-substrate distance was varied between 7 and $13 \mathrm{~cm}$.

The FWHM of the poles in the phi-direction are shown in figure VII.2.4.
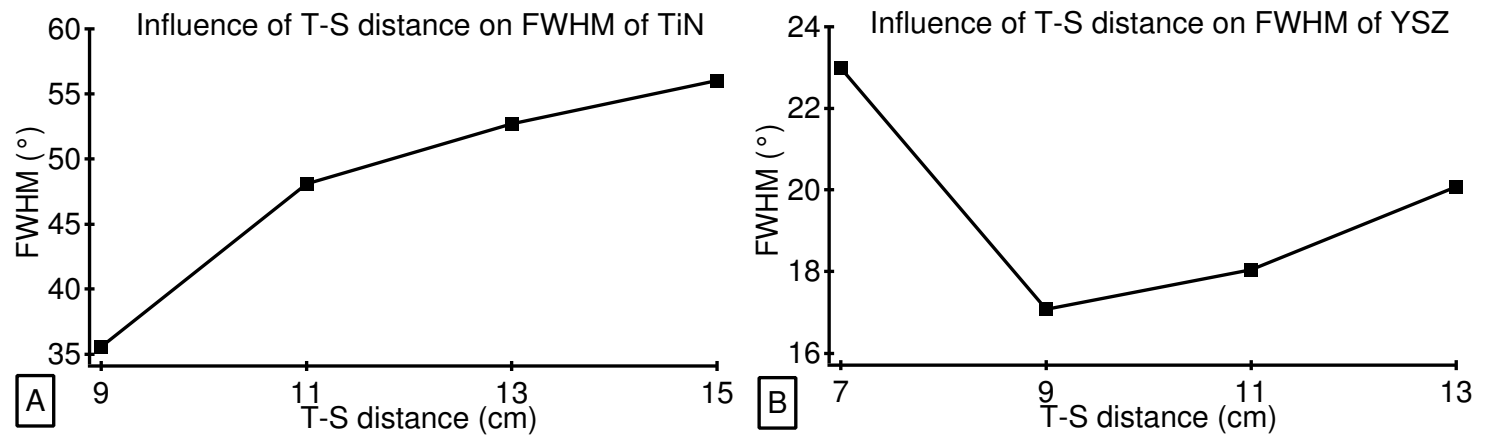

Figure VII.2.4: Influence of the target-substrate distance on the degree of in-plane alignment for TiN (a) and YSZ (b). For both materials, an improving in-plane alignment with decreasing target-substrate distance is observed. In case of YSZ, the in-plane alignment get worse at a too low T-S distance. In case of TiN, the $\mathrm{T}-\mathrm{S}$ distance was not decreased enough to observed a minimum.

In case of YSZ, the in-plane alignment improves with decreasing target-substrate distance, but decreasing the target-substrate distance too much results in a worse in-plane alignment. An optimum around a target-substrate distance of $9 \mathrm{~cm}$ is observed.

However, in case of TiN, the in-plane alignment improves with decreasing target-substrate distance down to $9 \mathrm{~cm}$, but no minimum was observed.

- Influence of the film thickness

Finally, also the influence of an increasing film thickness on the degree of in-plane alignment was investigated.

TiN thin films were deposited by a $1 / 9$ unbalanced magnetron on grounded polycrystalline stainless steel $\left(\mathrm{V}_{\mathrm{s}}=0 \mathrm{~V}\right)$, at a target-substrate angle of $55^{\circ}$, a pressure of $0.58 \mathrm{~Pa}$, a targetsubstrate distance of $13 \mathrm{~cm}$ and an $\mathrm{Ar} / \mathrm{N}_{2}$ ratio of 55/5. The deposition time was adapted to obtain a film thickness between 0.72 and $1.55 \mu \mathrm{m}$.
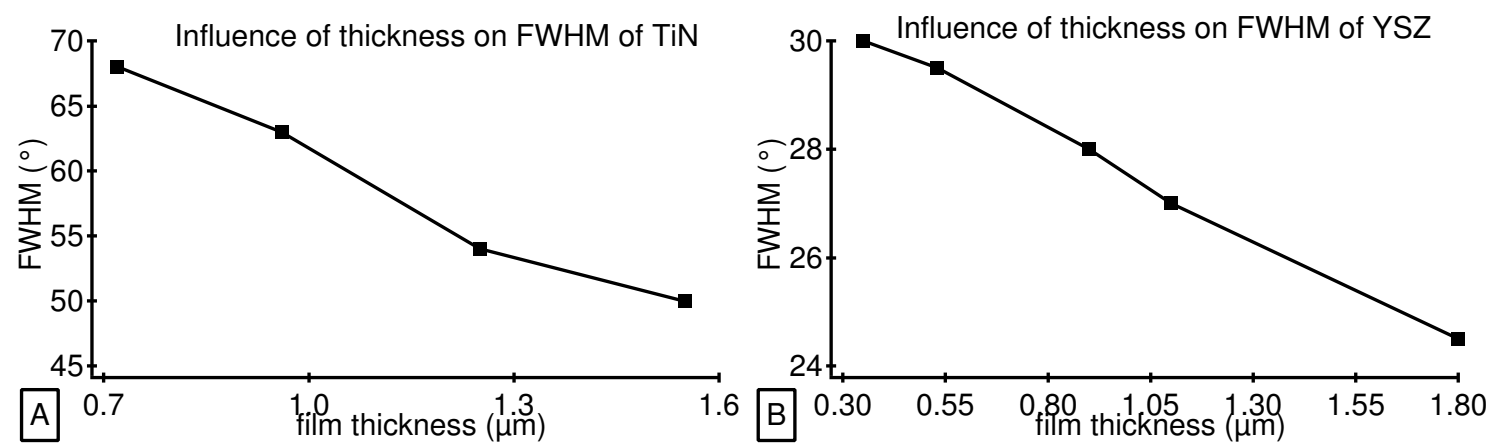

Figure VII.2.5: Influence of the film thickness on the degree of in-plane alignment for TiN (a) and YSZ (b). For both materials, an improving in-plane alignment with increasing film thickness is observed. 
It was reported in [1] that YSZ thin films were deposited by a 1/9 unbalanced magnetron on a grounded polycrystalline stainless steel $\left(\mathrm{V}_{\mathrm{s}}=0 \mathrm{~V}\right)$, at a target-substrate angle of $55^{\circ}$, a pressure of $0.8 \mathrm{~Pa}$, a target-substrate distance of $13 \mathrm{~cm}$ and an $\mathrm{Ar} / \mathrm{O}_{2}$ ratio of $60 / 2$. The film thickness was varied between 0.35 and $1.8 \mu \mathrm{m}$.

Similar to all previous depositions, all thin films were biaxially aligned and the FWHM of the poles in the phi-direction are shown in figure VII.2.5.

For both materials it is observed that the degree of in-plane alignment continuously improves with increasing film thickness.

As a first conclusion, it is observed that the in-plane alignment of YSZ and TiN behaves almost similar as a function of the substrate bias, target-substrate angle, pressure, targetsubstrate distance and film thickness. Beside the influence of these five parameters, also the influence of the target usage, the condition of the chamber walls, the $\mathrm{Ar} / \mathrm{O}_{2}$ ratio and the magnet configuration of the used magnetron has been investigated for YSZ.

- Influence of the $\mathrm{Ar} / \mathrm{O}_{2} 2$ ratio:

YSZ thin films of $\sim 1.3 \mu \mathrm{m}$ were deposited by a $1 / 9$ unbalanced magnetron on a positively biased polycrystalline stainless steel $\left(\mathrm{V}_{\mathrm{s}}=+15 \mathrm{~V}\right)$, at a target-substrate angle of $65^{\circ}$, a pressure of $0.3 \mathrm{~Pa}$ and a target substrate-distance of $9 \mathrm{~cm}$. Four depositions at different $\mathrm{Ar} / \mathrm{O}_{2}$ flow ratio's of 58/1.7, 58/2, 58/2.5 and 57/3 were carried out. $\mathrm{An}_{2}$-flow lower than $1.7 \mathrm{sccm}$ could not be used since this resulted in bad oxidized YSZ films. The influence of the increasing $\mathrm{O}_{2}$-flow on the in-plane alignment is shown in figure VII.2.6. It is observed that the degree of in-plane alignment improves with decreasing $\mathrm{O}_{2}$-flow. An optimal in-plane alignment of $14.8^{\circ} \mathrm{FWHM}$ in the phi direction was obtained. This was the best in-plane aligned YSZ film that could be obtained by the used deposition system up to now.

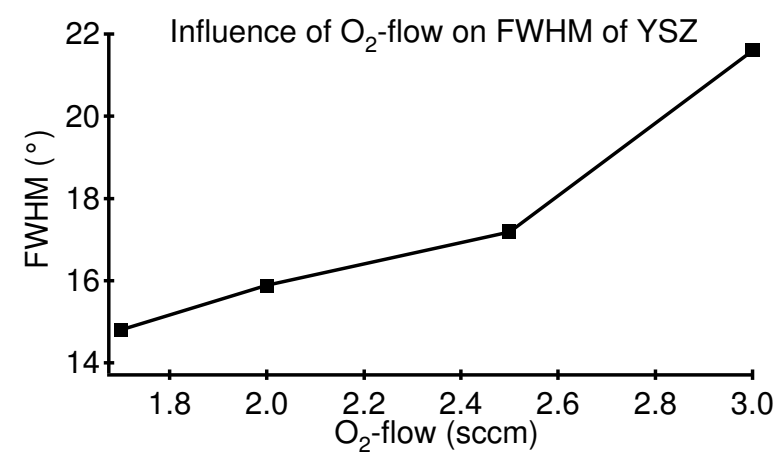

Figure VII.2.6: Influence of the $\mathrm{O}_{2}$-flow on the degree of in-plane alignment for YSZ. An improving inplane alignment with decreasing $\mathrm{O}_{2}$-flow is observed.

- Influence of the target usage:

As discussed in chapter III, all depositions are carried out by sputtering from a planar 5 $\mathrm{cm}$ diameter target. Due to the geometry of the used magnet set-up this results in the formation of an erosion profile, often called a race-track. Of course, the longer a target is used, the more pronounced that erosion profile. In case of $\mathrm{YSZ}$ deposition, a $\mathrm{Zr} / \mathrm{Y}$ target with a thickness of $4 \mathrm{~mm}$ was used. An experimental measured erosion profile of an almost completely used target is shown in figure VII.2.7.

The influence of the target usage on the degree of in-plane alignment has been investigated by depositing two YSZ films on complete identical conditions, but the first with a brand new target and the second with a completely used or "end of life" one. The depositions of $1.3 \mu \mathrm{m}$ thick YSZ films were carried out on a grounded polycrystalline stainless steel substrate, a target-substrate distance of $13 \mathrm{~cm}$, a pressure of $0.55 \mathrm{~Pa}$, a 
target-substrate angle of $55^{\circ}$ and an $\mathrm{Ar} / \mathrm{O}_{2}$ ratio of 58/2. The deposition with a new target resulted in a FWHM of $29.4^{\circ}$, while the deposition with the already completely used target results in a FWHM of $22.3^{\circ}$. Hence, it can be concluded that the more the target is used, or in other words the more the erosion profile is pronounced, the better the in-plane alignment of the films. Beside the improved in-plane alignment, an increasing target usage also results in a decreasing deposition rate. For the two upper mentioned depositions, the experimentally measured deposition rate $R_{d}$ decreased from $66.0 \mathrm{~nm} / \mathrm{min}$ to $39.3 \mathrm{~nm} / \mathrm{min}$ when changing from the new target to the completely used one.

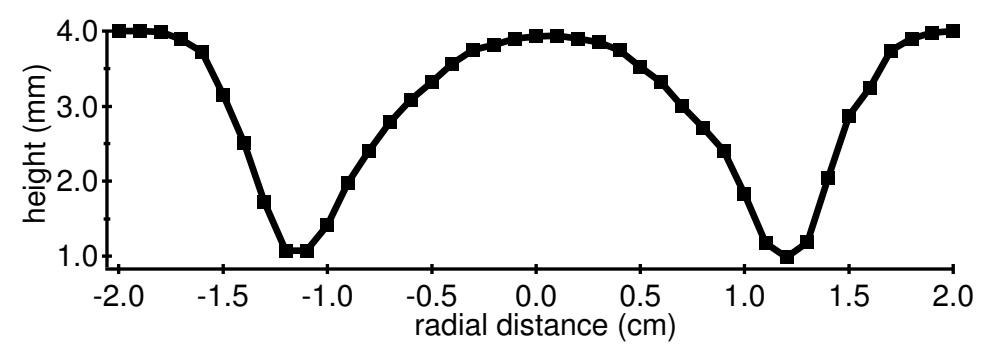

Figure VII.2.7: Experimental erosion profile of an almost completely used $4 \mathrm{~mm}$ thick $\mathrm{Zr} / \mathrm{Y}$ target.

On average, at least 40 YSZ thin films of 1 à1.5 $\mu \mathrm{m}$ could be deposited with one target. Hence, the influence of the target usage in a series of five depositions can be neglected in a first approximation since the difference between the first and fifth deposition should be less than $1^{\circ}$ in FWHM due to the target usage.

- Influence of the condition of the chamber walls:

YSZ is an electrical insulator, hence depositing YSZ on the substrate, and thus also on the chamber walls, causes these walls in the long run to become also insulating. As already discussed in chapter $\mathrm{V}$, depositing the chamber walls with an insulating material causes these walls to become electrically floating. This causes a rather drastic change in some plasma characteristics, like the plasma potential $\mathrm{V}_{\mathrm{p}}$ and floating potential $\mathrm{V}_{\mathrm{f}}$. Also the possible influence of that chamber wall coating with an insulating material on the degree of in-plane alignment has been investigated. Therefore, two YSZ thin films of $1.15 \mu \mathrm{m}$ were deposited on a grounded polycrystalline stainless steel substrate, at a pressure of 0.65 $\mathrm{Pa}$, an $\mathrm{Ar} / \mathrm{O}_{2}$ ratio of $75 / 3.75$, a target-substrate angle of $55^{\circ}$ and a target-substrate distance of $13 \mathrm{~cm}$. The first deposition was ignited in a complete "clean" deposition chamber. Hence, all chamber walls were well conducting at the start of the deposition. The second deposition was started in a deposition chamber of which the walls were already fully coated with insulating YSZ. The deposition in the situation of conducting chamber walls resulted in a FWHM of $33.9^{\circ}$ in the phi-direction, while in case of the deposition in a chamber with insulating walls resulted in a FWHM of only $24.2^{\circ}$ in the phi direction. Hence, it can be concluded that the condition of the chamber walls has a large influence on the degree of in-plane alignment. All former reported YSZ depositions were carried out in an already fully YSZ coated deposition chamber, and thus in the situation of insulating walls.

- Influence of the magnet configuration:

Finally, also the influence of the used magnet array in the magnetron was investigated. As discussed in chapter III, nearly all deposition were carried out with an unbalanced magnetron, in which the total magnet strength of the outer magnet is 9 times stronger than the total magnet strength of the inner magnet. Such a magnetron is called a $1 / 9$ unbalanced magnetron, and results an a high density plasma plume up to the substrate, as discussed in chapter III (section III.3). To investigate the influence of the degree of that unbalancing of the magnetron, also a deposition with a less unbalanced magnetron was carried out. This 
means that a magnetron in which the total strength of the outer magnet is only 3 times larger than the total strength of the inner magnet was used. Two YSZ depositions of 1.5 $\mu \mathrm{m}$ were carried out on a grounded polycrystalline stainless steel substrate, at a pressure of $0.55 \mathrm{~Pa}$, a target-substrate distance of $13 \mathrm{~cm}$, a target-substrate angle of $55^{\circ}$ and an $\mathrm{Ar} / \mathrm{O}_{2}$ ratio of $75 / 3.75$. The $\mathrm{YSZ}$ deposition with the $1 / 9$ unbalanced magnetron resulted in an in-plane alignment of $23.2^{\circ}$ in FWHM, while the deposition with a $1 / 3$ magnetron only resulted in a FWHM of $29.0^{\circ}$. Hence, the more the magnetron is unbalanced, the better the resulting in-plane alignment, at least for the deposition conditions under discussion.

After the investigation of all these parameters, the degree of in-plane alignment for TiN and YSZ could be optimized by choosing the right deposition conditions.

In case of YSZ, these optimized deposition conditions were a $+15 \mathrm{~V}$ biased substrate, a film thickness of $1.3 \mu \mathrm{m}$, a target-substrate distance of $9 \mathrm{~cm}$, a target-substrate angle of $65^{\circ}$, a pressure of $0.3 \mathrm{~Pa}$ and an $\mathrm{Ar} / \mathrm{O}_{2}$ ratio of $58 / 1.7 \mathrm{sccm}$ and resulted in an in-plane alignment of $14.8^{\circ}+/-0.7^{\circ}$.

In case of TiN, an in-plane alignment of $18.1^{\circ}+/-0.9^{\circ}$ in FWHM could be obtained for a deposition on $\mathrm{a}+15 \mathrm{~V}$ biased polycrystalline stainless steel substrate, a target-substrate distance of $9 \mathrm{~cm}$, an $\mathrm{Ar} / \mathrm{N}_{2}$ ratio of 55/5, a target-substrate angle of $60^{\circ}$, a pressure of $0.48 \mathrm{~Pa}$ and a film thickness of $1.7 \mu \mathrm{m}$.

\section{3: Discussion}

In order to understand why and how all these deposition parameters influence the degree of in-plane alignment, the proposed model in chapter VI should be reconsidered in detail. Two major assumptions or conditions had to be fulfilled to obtain an in-plane alignment.

First, it was shown that the resulting in-plane alignment was mainly caused by the $2 \mathrm{D}$ growth, i.e. the expansion of grains due to the incorporation of adparticles which first stroke on the bare substrate or on another grain and then diffused towards the expanding grain. As shown in figure VII.3.1, this imposes that all these adparticles are at least able to diffuse up to the edge of that grain.

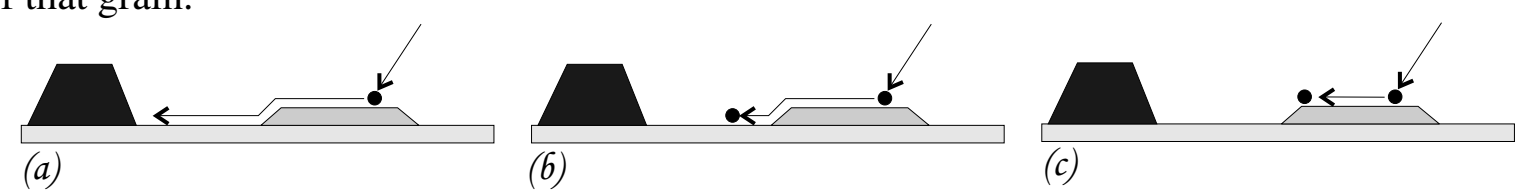

Figure VII.3.1: Schematic drawing in which it is shown that the completion of the 2D growth of the left grain imposes that the adparticles are able to reach the edge of that grain (a). In case that the net diffusion distance of these adparticles is not large enough (b) or in case that they can't cross the Erlich-Schwoebel barrier (c), these adparticles don't take part to the $2 \mathrm{D}$ growth of the left grain.

This means that the net diffusion distance, i.e. the distance between the place where the adparticles first hit the growing film and the final place where they become chemisorbed, must be large enough to obtain 2D growth and thus an in-plane aligned film. Also the overall mobility must be high enough to allow the diffusion from one grain to another grain, but can't be too high since re-structurative grain growth may not happen, i.e. zone T growth conditions. It can be concluded that the mobility of the adparticles is a first fundamental parameter influencing the resulting degree of in-plane alignment.

Second, the in-plane alignment was calculated to be a consequence of the in-plane growth rate anisotropy of the different grains due to the anisotropy in the amount of diffusing adparticles that these grains capture. This amount of diffusing adparticles captured by an YSZ grain was 
calculated as shown in figure VII.3.2. An analogous calculation was made for TiN grains, by treating triangular shaped instead of square shaped grains.

Important to note is that the diffusion direction of all adparticles was in a first approximation assumed to be along one single direction, i.e. the same direction for all adparticles. This assumption can only be valid if all incoming adparticles arrive on the growing film along the plane defined by the substrate normal and the target normal, as shown in figure VII.3.3.

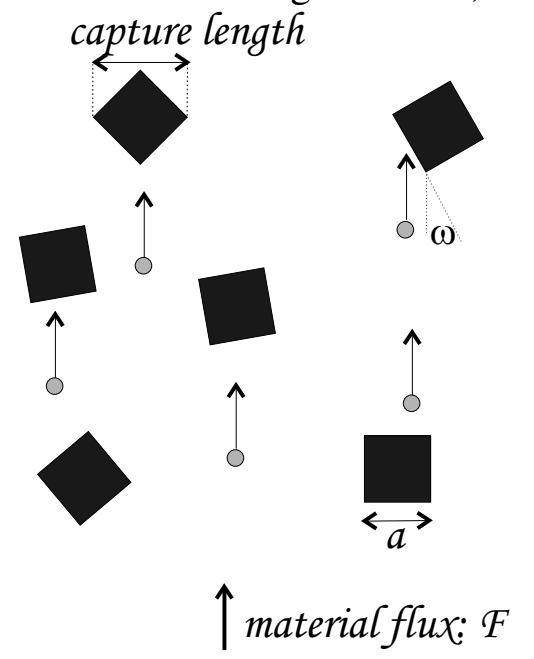

Figure VII.3.2: Schematic drawing of the 2D growth of square shaped grains. $\omega$ stands for the in-plane orientation. The arrow indicates the direction along it was assumed that all adparticles diffuse.

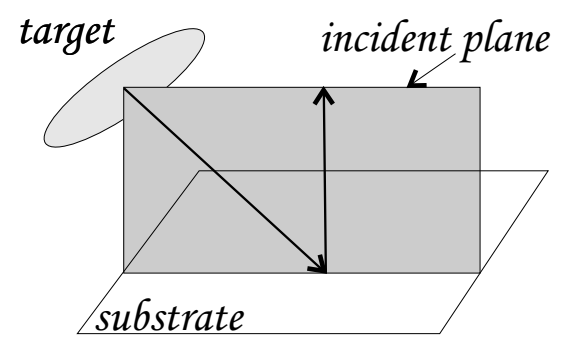

Figure VII.3.3: Schematic drawing of the plane defined by the substrate normal and the target normal. In the model presented in chapter VI, it was assumed that all adparticles arrive on the film parallel to that plane.

However, for most physical vapour deposition techniques, as e.g. planar magnetron sputtering, this is not the case. This because the particles are sputtered from a limited sized target and also had to pass the gas phase before they hit the substrate. During this transport through the gas phase, these sputtered particles can collide with other particles and hence can loose their direction. This causes the incoming material flux on the substrate not to be along one single direction, but having a certain angular spread.
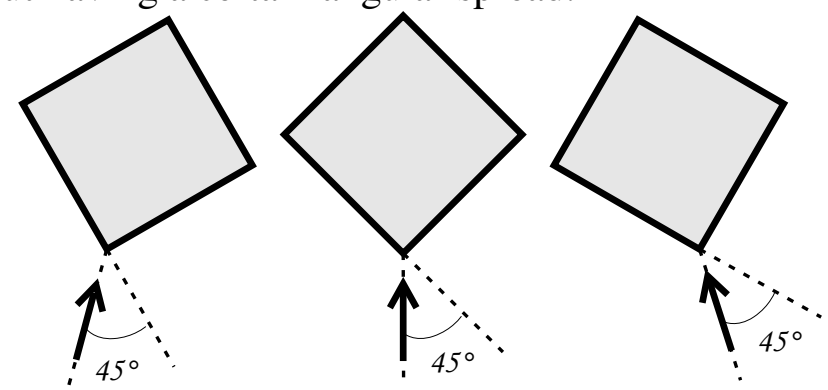

Figure VII.3.4: Influence on the degree of in-plane alignment of the angular spread on the incoming material flux. The arrow indicates the direction of the incoming material flux and the grey square shaped grains are all oriented along the right, i.e. $\omega=45^{\circ}$, in-plane orientation.

As shown in figure VII.3.4, this angular spread will impose a lower limit on the obtainable degree of in-plane alignment, because such an angular spread implies that several square 
shaped YSZ grains with different in-plane orientation will lie along the right direction, i.e. $\omega$ $=45^{\circ}$. In an analogous way, it can be shown that also in case of triangular shaped TiN grains, an angular spread on the incoming material flux will impose a lower limit on the obtainable in-plane alignment.

Hence, even in case that the net diffusion distance of the adparticles is large enough, and in case of an optimal adparticle mobility, no perfect in-plane alignment will develop since it is limited by the angular spread on the incoming material flux. It can be concluded that this angular spread is a second fundamental parameter influencing the degree of in-plane alignment.

It is clear that several deposition parameters like e.g. the target-substrate distance, pressure, target-substrate tilt angle will influence the angular spread on the incoming material flux and thus will influence the final degree of in-plane alignment.

Unfortunately, that angular spread on the incoming material flux is not easy to measure. Several groups have build up a system to measure that angular spread experimentally [2,3], but their measurements only have a resolution of some degrees. As seen from the experimentally measured FWHM of the in-plane alignment as a function of the deposition parameters (section VII.2) the in-plane alignment often only varies with $1^{\circ}$ à $2^{\circ}$ in FWHM. Hence, the angular spread of the incoming material flux should also be known at least with a certainty of $1^{\circ}$. Instead of building an expensive and complicated measuring system, a binary collision Monte Carlo simulation package has been written to simulate the incoming material flux.

\section{VII.4: Description of the Monte Carlo model}

Simulating the material flux towards the substrate needs the knowledge of the initial properties when the sputtered particles leave the target and the simulation of the transport through the gas phase. Therefore, the program is divided in two parts: sputtering from the target, i.e. simulation of the nascent flux and transport through the gas phase.

\section{VII.4.1: Nascent sputter flux:}

In order to know the nascent sputter flux, as well the position where a sputtered particle leaves the target, as its energy and direction should be known.

\section{- Position:}

As already discussed in chapter III, sputtering from a planar magnetron causes the formation of an erosion profile or a race-track in the target. A measured erosion profile of a completely used target sputtered from a 1/9 unbalanced magnetron is shown in figure VII.2.7, while a schematic drawing is seen in figure VII.4.1.

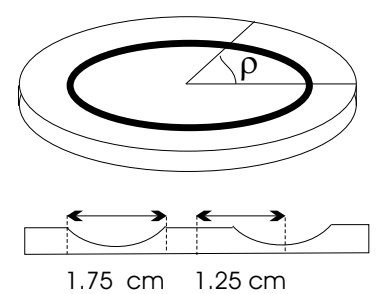

Figure VII.4.1: Sketch of the formed erosion profile or race-track.

This erosion profile is a direct representation of the distribution of the radial distance $\mathrm{R}$ from the centre of the target at which a particle is sputtered. For the simulation, this distribution of the radial distance $\mathrm{R}$ has been approximated by a Gauss distribution:

$$
P(R)=\frac{1}{\sigma \sqrt{2 \pi}} e^{\frac{-(R-\mu)^{2}}{2 \sigma^{2}}} \text { (eq.VII.4.1) }
$$


The radius of the experimental race-track is $1.25 \mathrm{~cm}$ (which is used for the mean $\mu$ of the gauss distribution) and the width of the race-track is $1.75 \mathrm{~cm}$ (from which the standard deviation is calculated: $3 \sigma=1.75 / 2)$. Hence $P(R)$ gives the probability that a particle is sputtered from the distance $\mathrm{R}$ from the centre of the target.

To know the exact position at which the sputtered particle leaves the target, also the radial angle $\rho$ should be known. This radial angle $\rho$ is random between 0 and $2 \pi$ since the race-track formation in a planar circular magnetron is radial symmetric.

\section{- Energy and direction:}

A lot of authors who also developed a model to simulate the sputtered flux used an energy independent nascent angular distribution according to the cosinus-law derived by Sigmund and the energy distribution according to the Thompson law [4-18]. As well as the cosinus-law derived by Sigmund as the energy distribution according to the Thompson law has been described in detail in chapter III. However, these distributions can only be valid in case that the energy of the incident ion on the target is high enough to cause a complete isotropic collision cascade, i.e. the linear cascade regime as defined in chapter III.

In case of magnetron sputtering, the energy of the incident ion on the target is around $400 \mathrm{eV}$ or even less, what causes the collision cascade to be not complete isotropic [19]. It follows that the angular distribution is not energy independent or that the angular and energy distribution of the sputtered particles are not separable [20-22].

Several binary collision Monte Carlo packages, e.g. ACAT [21], SRIM [23] and TRIM [24] has been written to simulate the energy and angular distribution of the sputtered particles independent from each other, avoiding the problems of the Sigmund-Thompson model. SRIM has been used to simulate the energy of $2 \times 10^{5}$ particles sputtered due to the impact of $400 \mathrm{eV}$ $\mathrm{Ar}^{+}$ions on the target. TRIM has been used to simulate the energy dependent angular distribution of the sputtered particles. The energy of the $\mathrm{Ar}^{+}$ions is again fixed at $400 \mathrm{eV}$. Using these TRIM results, angular distributions of sputtered particles with an energy in the intervals $0-8,8-20,20-40,40-60,60-80,80-100$ and $100-400 \mathrm{eV}$ has been fitted by $5^{\text {th }}$ order polynomials. In figure VII.4.2, the simulated and fitted angular distributions for $\mathrm{Zr}$ sputtered by $400 \mathrm{eV} \mathrm{Ar}^{+}$ions are shown. It is observed that the angular distribution strongly depends on the energy of the particles, what confirms that the Sigmund-Thompson distribution can't be used.

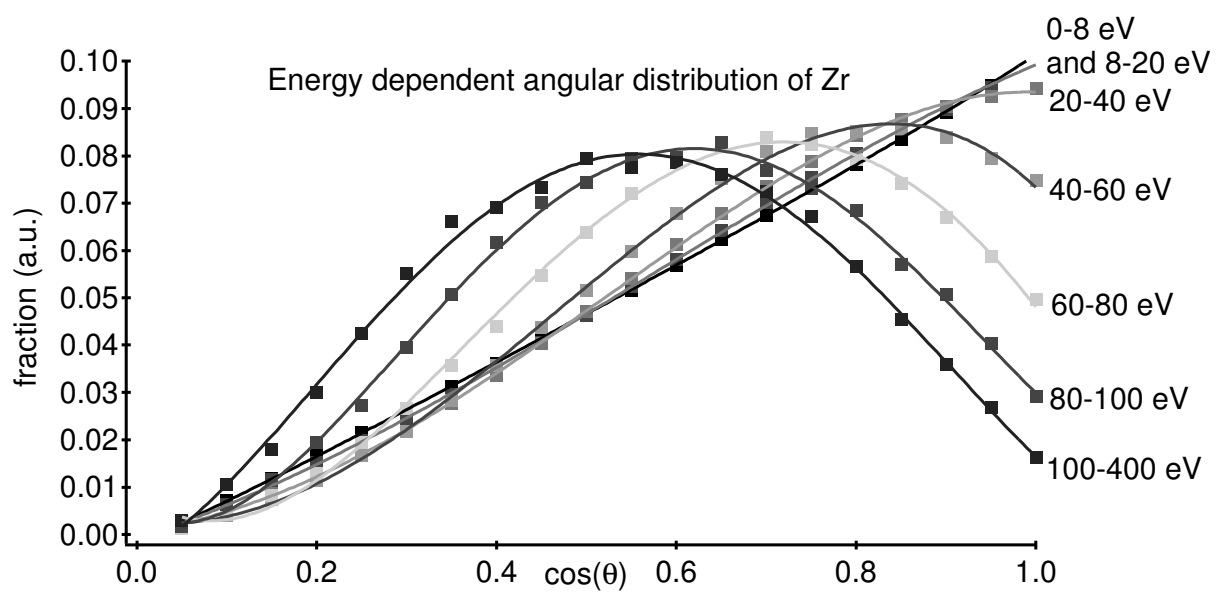

Figure VII.4.2: Simulated and fitted angular distribution function of $\mathrm{Zr}$ atoms, sputtered by $400 \mathrm{eV} \mathrm{Ar}^{+}$ions for several energy intervals.

Since the discharge voltage $\mathrm{V}_{\mathrm{d}}$, and thus also the energy of the impinging $\mathrm{Ar}^{+}$ions on the target, changes with changing deposition conditions, the influence of a variation in the $\mathrm{Ar}^{+}$ion 
energy on the energy and angular distribution should be checked. This has been done for $\mathrm{Ar}^{+}$ ions in the energy range of 300 to $600 \mathrm{eV}$. As seen in figure VII.4.3 and VII.4.4, no real variation in the energy and angular distribution was observed.

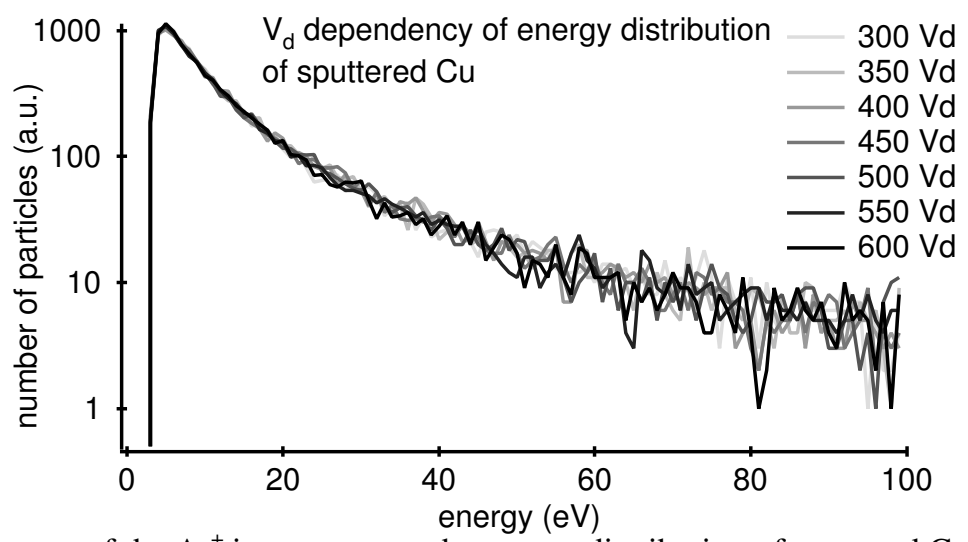

energie (Ev)

Figure VII.4.3: Influence of the $\mathrm{Ar}^{+}$ion energy on the energy distribution of sputtered $\mathrm{Cu}$ particles. No strong influence is observed.

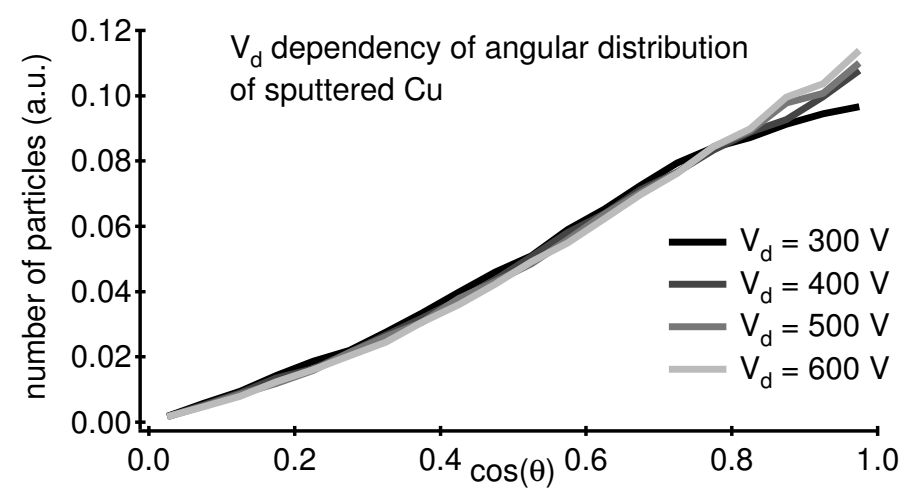

Figure VII.4.4: Influence of the $\mathrm{Ar}^{+}$ion energy on the angular distribution of sputtered $\mathrm{Cu}$ particles. No strong influence is observed.

Knowing the energy of the sputtered particles, the polar ejection angle $\theta$ of each particle was chosen according to the corresponding calculated angular distribution. The azimutal ejection angle $\phi$ is chosen random between 0 and $2 \pi$.

In this way, the initial place ( $R$ and $\rho$ ), the initial energy $E$ and the initial direction $(\theta$ and $\phi)$ of a sputtered particle was obtained. This has been done for $2 \times 10^{5}$ sputtered particles, what gives a complete set for the nascent sputter flux. When the simulation program is running, a random particle is chosen from this set and is launched from the target.

VII.4.2: Transport through the gas phase:

Once the sputtered particle is in the gas phase, collisions may occur. It is assumed that the sputtered particles will only collide with the argon atoms (and not with other sputtered particles). This assumption is justified because the concentration of sputtered particles $\left(\mathrm{n}_{\mathrm{s}}\right)$ is very small compared to the concentration of the background gas $\left(\mathrm{n}_{\mathrm{Ar}}\right)$ at the used conditions $\left(\mathrm{n}_{\mathrm{s}} / \mathrm{n}_{\mathrm{Ar}}\right.$ is $10^{-2}$ à $\left.10^{-3}\right)$. Secondly, it is assumed that the distribution of the background gas is constant in time and space, what ignores the pressure variations which may occur during sputtering at higher pressures and sputtering power due to gas heating [25,26]. Thirdly, we neglect charged particles (ions, electrons) and non-elastic interactions because ion and electron concentrations are small in comparison with the atom quantity (typical ionization degree is $10^{-3}$ à $10^{-4}$ [27]). Finally, for similar reasons only sputtered atoms were considered, and no multi-atomic particles [28]. 
To simulate the collisional transport through the gas phase, first the distance between two collisions, i.e. the free path of the particle should be known. This distance is related to the mean free path. If a collision occurs, the particle will loose some energy and will also change its direction. Hence, a new energy and new direction has to be calculated. This collisional transport should be followed up till the particle reaches the substrate, one of the chamber walls or get thermalized. As soon as a particle is considered to be thermalized, it was assumed that it will continue its trajectory by a random walk until it reaches the substrate or the chamber walls. The visual basic codes written to simulate this transport can be found at the end of this chapter as appendix.

\section{- Free path:}

The ejected metallic particles travel a distance $\lambda^{*}$ in a straight line between collisions with gas atoms. The length of the free path between collisions, $\lambda^{*}$, is determined stochastically from a Poisson distribution according to a standard Monte Carlo procedure: $\lambda^{*}=\lambda \ln (1 / 1-\mathrm{x})$ where $\mathrm{x}$ is a random number from the interval $(0,1)$ and $\lambda$ the mean free path of an atom.

From the kinetic gas theory [29] the mean free path $\lambda$ of an atom, with mass $M_{s}$, traveling through a gas with mass $M_{g}$ is

$$
\lambda^{-1}=4 \sqrt{2} \pi \frac{P_{s}}{k T} r_{s}^{2}+\pi \frac{P_{g}}{k T}\left(r_{s}+r_{g}\right)^{2}\left(1+\frac{M_{s}}{M_{g}}\right)^{1 / 2} \quad \text { (eq. VII.4.2) }
$$

with $P_{s}$ and $P_{g}$ the partial pressures and $r_{s}$ and $r_{g}$ the atom radius of the sputtered and gas atoms respectively. As mentioned earlier, the density of the sputtered atoms is much less than the density of the background gas, hence the first term in eq. VII.4.2 can be neglected.

Robinson [30] has shown that this mean free path is also energy dependent and an empirical fit showed that the mean free path $\lambda$ should be multiplied with a factor $E^{a}$ with $E$ the energy of the sputtered atoms in $\mathrm{eV}$ and the exponent $\mathrm{a}=0$ (for $\mathrm{E}<1 \mathrm{eV}$ ) or 0.29 (for $\mathrm{E}>1 \mathrm{eV}$ ). This results in an energy dependent mean free path as given in eq. VII.4.3.

$$
\lambda=E^{a} /\left(\pi \frac{P_{g}}{k T}\left(r_{s}+r_{g}\right)^{2}\left(1+\frac{M_{s}}{M_{g}}\right)^{1 / 2}\right)
$$

If the energy of the sputtered particle is known, the mean free path $\lambda$ can be calculated by eq.VII.4.3. Then, the actual free path is determined stochastically from the Poisson distribution $\lambda^{*}=\lambda \ln (1 / 1-\mathrm{x})$, with $\mathrm{x}$ in the interval $(0,1)$. Knowing the free path $\lambda$ and the direction of the particle, it is known where it collides with Ar.

Table VII.4.5 gives the used values for the atom radius and mass of the simulated materials and Ar. These materials were chosen, since they were used by the group during the research to the mechanism of biaxial alignment.

Table VII.4.5: Mass and radius of the used materials and Ar. The radius was the metallic radius.

\begin{tabular}{|l|l|l|}
\hline material & Mass (a.m.u.) & Radius (nm) \\
\hline $\mathrm{Ti}$ & 47.9000 & 0.147 \\
\hline $\mathrm{Zr}$ & 91.2200 & 0.160 \\
\hline $\mathrm{Y}$ & 88.9050 & 0.178 \\
\hline $\mathrm{Cu}$ & 63.5400 & 0.138 \\
\hline $\mathrm{Al}$ & 26.9815 & 0.143 \\
\hline $\mathrm{Ag}$ & 107.870 & 0.144 \\
\hline $\mathrm{Mg}$ & 24.3120 & 0.160 \\
\hline $\mathrm{Cr}$ & 51.9960 & 0.130 \\
\hline $\mathrm{Ar}$ & 39.9480 & 0.102 \\
\hline
\end{tabular}




\section{- Collision with Ar:}

If a sputtered particle collides with Ar, it will loose energy and change its direction, thus the scattering angle and the energy loss should be calculated. A schematic sketch of the collision and all used symbols are shown in figure VII.4.6.

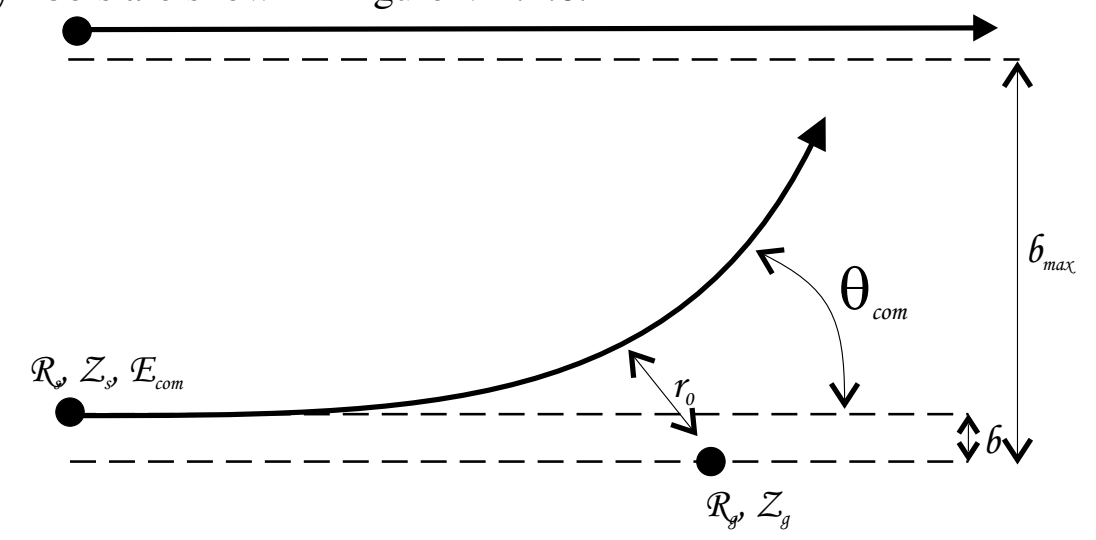

Figure VII.4.6: Schematic drawing of the collision of a sputtered particle (s) with an $\mathrm{Ar}(\mathrm{g})$. All used symbols are mentioned. The subscript "com" stands for the Centre of Mass reference system. The lower sputtered particle collides with Ar, while the upper does not.

An interaction potential between the sputtered particle and the Ar has to be taken into account. Here, a spherically symmetric atom-atom interaction potential was used. More specific, a screened Coulomb potential with the Molière screening function and the Firsov screening length [24] as given in eq. VII.4.4 and VII.4.5, with $Z_{s}$ and $Z_{g}$ the atomic number of the sputtered atom and the background gas respectively and $r$ the inter-atomic distance.

$$
\begin{aligned}
& V(r)=\frac{Z_{s} Z_{g} e^{2}}{r} g(r) \quad(\text { eq.VII.4.4) with } \\
& g(r)=0.35 e^{-0.3 \frac{r}{a_{s}}}+0.55 e^{-1.2 \frac{r}{a_{s}}}+0.1 e^{-6 \frac{r}{a_{s}}} \text { and } a_{s}=0.468 /\left(\sqrt{Z_{s}}+\sqrt{Z_{g}}\right)^{0.668} \quad \text { (eq. VII.4.5) }
\end{aligned}
$$

For such a spherically symmetric atom-atom interaction potential, the relative scattering angle in the centre of mass frame is given by

$$
2 \theta_{\text {com }}=\pi-2 b \int_{r_{0}}^{\infty} \frac{d r}{r^{2}\left\{1-\left[V(r) / E_{c o m}\right]-\left(b^{2} / r^{2}\right)\right\}^{1 / 2}} \quad \text { (eq.VII.4.6) }
$$

in which $r_{0}$ is implicitly determined by

$$
b^{2}=r_{0}^{2}\left(1-\frac{V\left(r_{0}\right)}{E_{c o m}}\right) \text { (eq.VII.4.7), }
$$

with $b$ the impact parameter between 0 and $b_{\max }, r$ the inter-atomic distance, $V(r)$ the interaction potential, $E_{c o m}$ the energy of the sputtered particle in the centre of mass frame and $r_{0}$ the minimal inter-atomic distance. The physical meaning of this impact parameter $b$ is drawn in figure VII.4.6. It seems logic, that the larger the impact parameter $b$, the weaker the interaction between the sputtered particle and the Ar. Hence, at high values of the impact parameter $b$, the sputtered particle is not anymore deflected by the Ar particle. As such, at high values of $b$, it can said that no collision happened. An arbitrary maximum value of $b$ should be chosen at which it is considered that the sputtered particle still collides with Ar. Here, the model uses a maximum value of $b\left(\right.$ i.e. $\left.b_{\max }\right)$ which is the smallest impact parameter at which the scattering angle $\theta_{\text {com }}$ is less than $0.286^{\circ}(0.005 \mathrm{rad})$. If the impact parameter $b$ is higher, the sputtered particle is considered to not collide with Ar.

Finally, the scattering angle $\theta_{\text {com }}$ gives the angle between the initial direction of the sputtered particle and the direction after collision, as shown in figure VII.4.7. Since the calculation of the scattering angle $\theta_{\text {com }}$ as a function of the energy $\mathrm{E}_{\mathrm{com}}$ and the impact parameter $b$ is rather 
intensive, a two-dimensional matrix of $b, E_{\text {com }}$ and $\theta_{\text {com }}$ has been calculated for all simulated materials and stored into separate files. Although the scattering angle $\theta_{\text {com }}$ is a continuous function of the energy $E_{c o m}$, it was calculated for some discrete values, i.e. $E_{c o m}$ is 0.03 , $0.04, \ldots, 0.99,1.00,1.1, \ldots 9.9,10,11, \ldots, 300 \mathrm{eV}$. This scattering angle $\theta_{\text {com }}$ as a function of the impact parameter $b$ for several energies $E_{c o m}$ is shown in figure VII.4.7. It is observed that this scattering angle decreases with increasing impact parameter $b$, and that this decrease is stronger the larger the energy $E_{c o m}$.

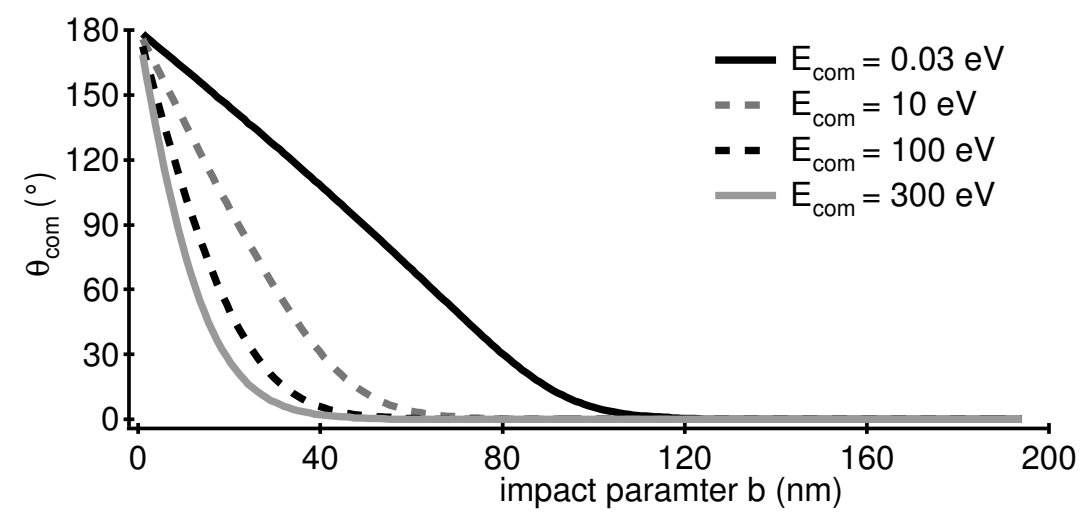

Figure VII.4.7: The scattering angle $\theta_{\text {com }}$ as a function of the impact parameter $b$ for several energies $\mathrm{E}_{\text {com }}$ during the collision of $\mathrm{Al}$ and thermal Ar.

- New direction:

Knowing the polar scattering angle $\theta_{c o m}$ in the centre of mass reference system, the polar scattering angle in the laboratory frame $\theta_{l a b}$ can be calculated by:

$$
\theta_{l a b}=\operatorname{Arctg}\left(\frac{\sin \theta_{c o m}}{\cos \theta_{c o m}+M_{s} / M_{g}}\right) \quad \text { eq. VII.4.8. }
$$

The azimuthal scattering angle $\phi_{l a b}$ is random between 0 and $2 \pi$ because the interaction potential is spherically symmetric.

To know the new direction of the sputtered particle, the scattering direction $\left(\theta_{l a b}\right.$ and $\left.\phi_{l a b}\right)$ should be added to the original direction $(\theta$ and $\phi)$ as schematically shown in figure VII.4.8 what finally results in the new direction of the sputtered particle $\left(\theta_{\text {new }}\right.$ and $\left.\phi_{\text {new }}\right)$.

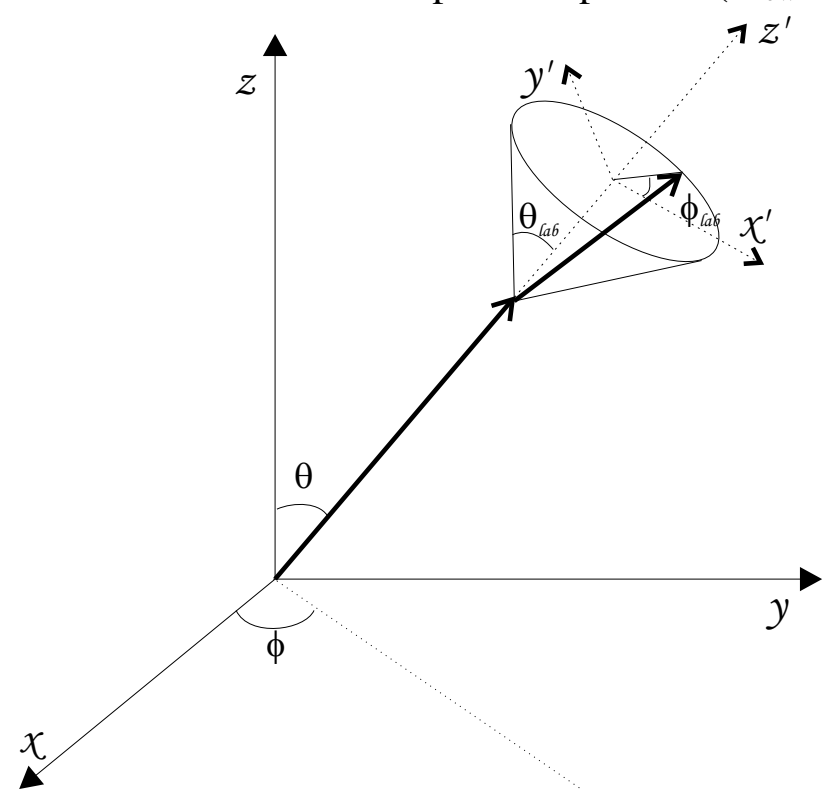

Figure VII.4.8: Definition of the original $(\theta$ and $\phi)$ and the additional direction $\left(\theta_{l a b}\right.$ and $\left.\phi_{l a b}\right)$ which has to be added in order to obtain the new direction. 
This addition of two directions is done by the following matrix multiplication:

$$
\left(\begin{array}{c}
\sin \left(\theta_{\text {new }}\right) \cos \left(\phi_{\text {new }}\right) \\
\sin \left(\theta_{\text {new }}\right) \sin \left(\phi_{\text {new }}\right) \\
\cos \left(\theta_{\text {new }}\right)
\end{array}\right)=\left(\begin{array}{ccc}
\cos (\theta) \cos (\phi) & -\sin (\phi) & \sin (\theta) \cos (\phi) \\
\cos (\theta) \sin (\phi) & \cos (\phi) & \sin (\theta) \sin (\phi) \\
-\sin (\theta) & 0 & \cos (\theta)
\end{array}\right) \times\left(\begin{array}{c}
\sin \left(\theta_{\text {lab }}\right) \cos \left(\phi_{\text {lab }}\right) \\
\sin \left(\theta_{\text {lab }}\right) \sin \left(\phi_{\text {lab }}\right) \\
\cos \left(\theta_{\text {lab }}\right)
\end{array}\right)
$$

In this way, the new direction in which the sputtered particle moves is known.

\section{- New energy:}

After collision with an Ar atom, the sputtered particle has lost energy, and the remaining energy is calculated by:

$$
E_{\text {labnew }}=E_{\text {lab }}\left(1-\frac{2\left(1-\cos \theta_{\text {com }}\right) M_{g} M_{s}}{\left(M_{s}+M_{g}\right)^{2}}\right) \quad \text { eq. VII.4.9 }
$$

After each collision, the new energy $E_{\text {com }}$ is compared with a chosen value $(0.03 \mathrm{eV})$. If it turns out that the particle has an energy lower than $0.03 \mathrm{eV}$, the particle is assumed to be thermalized. Then another simulation program is started, in which this particle continues its trajectory with a random walk until it hits the walls or the substrate. This means that the particle will collide every mean free path, after which its new direction is fully random.

VII.4.3: Analysis of incoming material flux:

The simulation volume is a box with dimensions $50 \mathrm{~cm} \times 50 \mathrm{~cm} \times \mathrm{d}(\mathrm{T}-\mathrm{S}) \mathrm{cm}$, in which $\mathrm{d}(\mathrm{T}-\mathrm{S})$ stands for the simulated target-substrate distance. This box geometry is shown in figure VII.4.9.

An atom leaves the target with an energy and direction as described in VII.4.1, after which it undergoes collisions (according to the description in VII.4.2). A sticking coefficient of one is assumed, thus the collisional transport is stopped when the atom reaches the walls or the substrate after which its properties (position, direction, energy and number of collisions) are stored in separate files. Next, another atom is launched from the target (until $5 \times 10^{5}$ atoms are sputtered).

The thickness profiles and all properties of the atoms arriving at the substrate are calculated from the stored data.

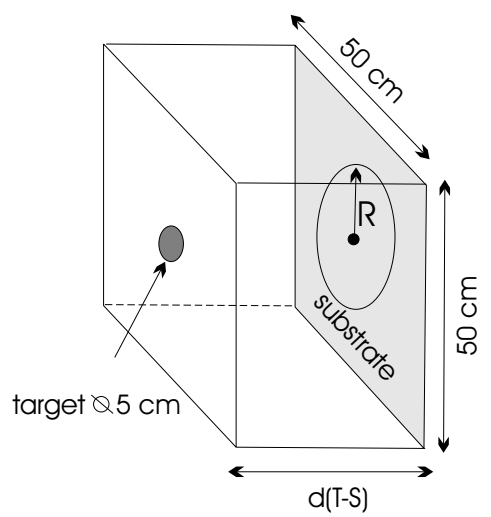

Figure VII.4.9: Sketch of the box in which the simulations were performed. A box with dimensions of 50x50xd(T-S) cm was used. All simulations were carried out for a $5 \mathrm{~cm}$ diameter target. $\mathrm{d}(\mathrm{T}-\mathrm{S})$ stand for the target-substrate distance, $\mathrm{R}$ stand for the radial distance from the centre of the substrate.

\section{VII.5: Validation of simulation code}

Of course, the written simulation program had to be checked. Since it is not trivial to measure the energy and the direction of the sputtered particles, the deposition profiles have been measured. If the experimental deposition profile corresponds to the simulated profile, it can be 
assumed that also the simulated energy and direction of the sputtered particles is correct, since both influence the transport and thus the deposition profile.

Secondly, it was also measured how the addition of a reactive gas influences the transport of the sputtered particles. This was necessary since all biaxially aligned thin films were deposited by reactive magnetron sputtering, i.e. in the presence of $\mathrm{Ar}$ and a reactive gas $\left(\mathrm{O}_{2}\right.$ or $\mathrm{N}_{2}$ ), while the above described model does not take into account the possible influence of the reactive gas on the nascent sputter flux and the collisional transport.

VII.5.1: Basic results and comparison of experimental and simulated deposition profiles: Thickness profiles of sputter deposited $\mathrm{Al}, \mathrm{Cu}$ and $\mathrm{Zr} / \mathrm{Y}(85 / 15$ weight $\%)$ have been measured experimentally for several pressures and distances. Therefore, a quartz crystal microbalance (Maxteck, Inc. Thickness monitor model TM-350) was mounted on a linear motion feedthrough. This allowed a vertical height variation of the quartz oscillator between -20 and $+20 \mathrm{~cm}$ with respect to the centre of the target (see figure VII.5.1).

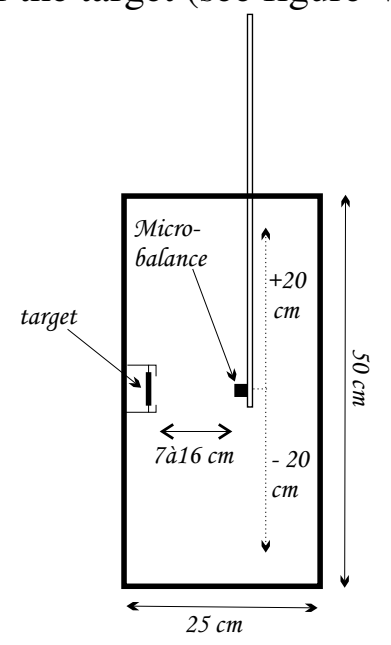

Figure VII.5.1: Experimental setup to measure the deposition profile.

The variation with pressure and target-substrate distance of the sputtered flux on the substrate for $\mathrm{Al}, \mathrm{Cu}$ and $\mathrm{Zr} / \mathrm{Y}(85 / 15$ weight $\%)$ has been simulated for $5 \times 10^{5}$ particles.

The flux of $\mathrm{Zr} / \mathrm{Y} 85 / 15$ has been calculated by simulating $4.2 \times 10^{5} \mathrm{Zr}$ particles, adding up the results for $0.8 \times 10^{5} \mathrm{Y}$ particles. Hence, the difference in atomic weight and sputter yield is taken into account. The results of these simulated thickness profiles, assuming a sticking coefficient of one and a constant density, together with the experimentally measured profiles are shown in figure VII.5.2, 5.3 and 5.4.

In case of the target-substrate variation, the filled symbols represent the experimental data and the open symbols the simulated data. However, in case of pressure variation, the symbols are not shown for clarity. Here, the solid lines correspond to the simulated data, while the dashed lines represent the experimental data.

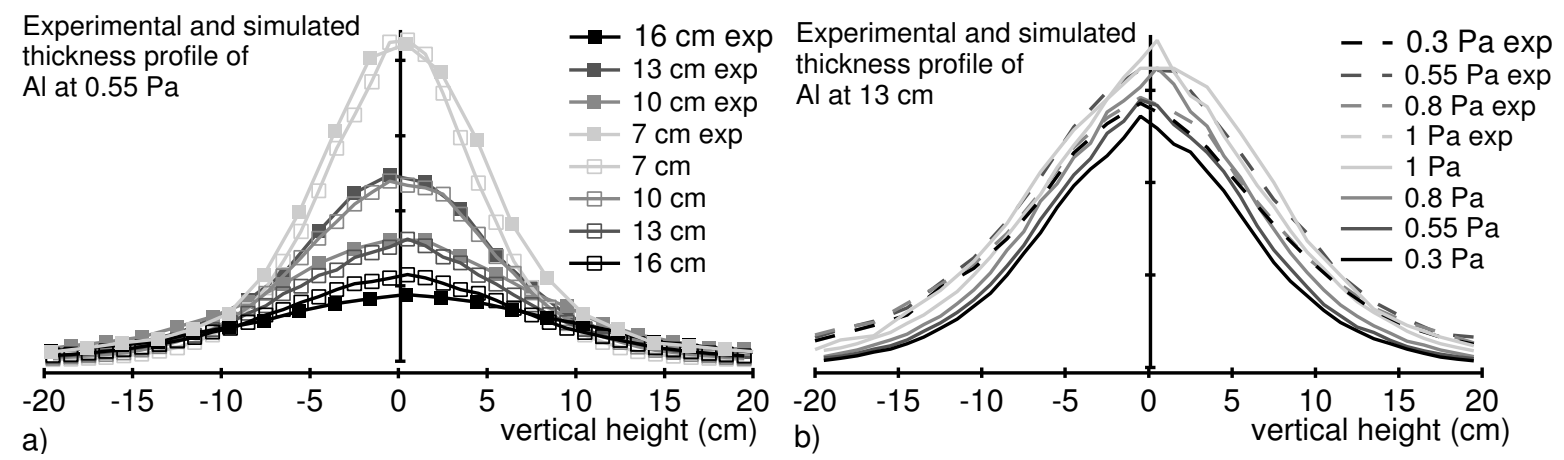

Figure VII.5.2: Simulated and experimental thickness profile of $\mathrm{Al}$ as a function of the target-substrate distance (a) and the pressure (b). 

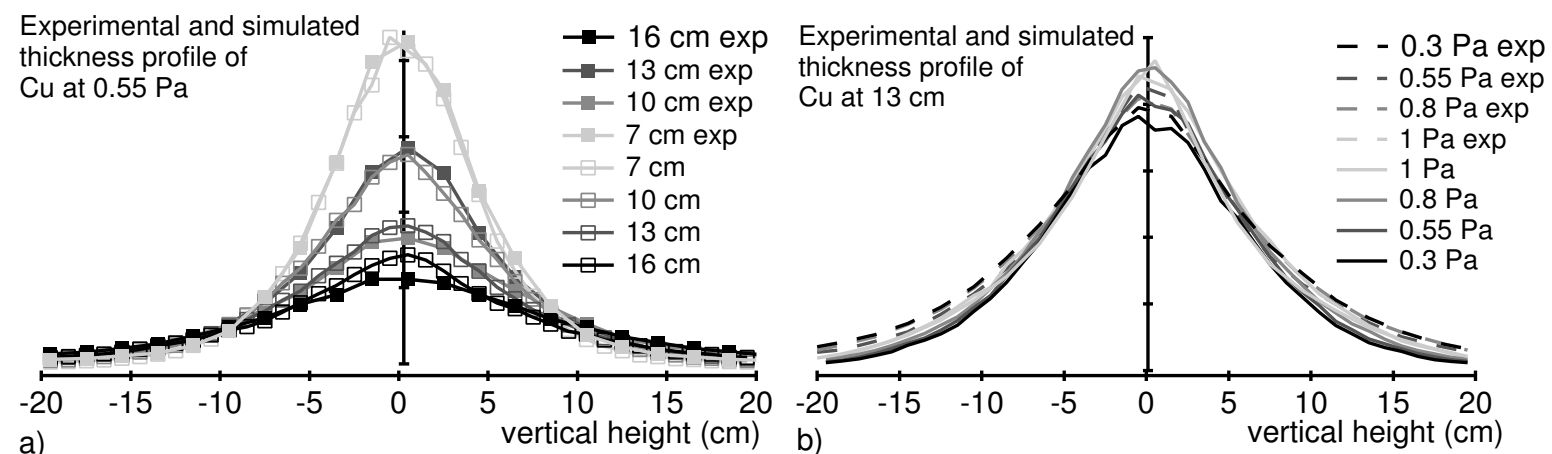

Figure VII.5.3: Simulated and experimental thickness profile of $\mathrm{Cu}$ as a function of the target-substrate distance (a) and the pressure (b).
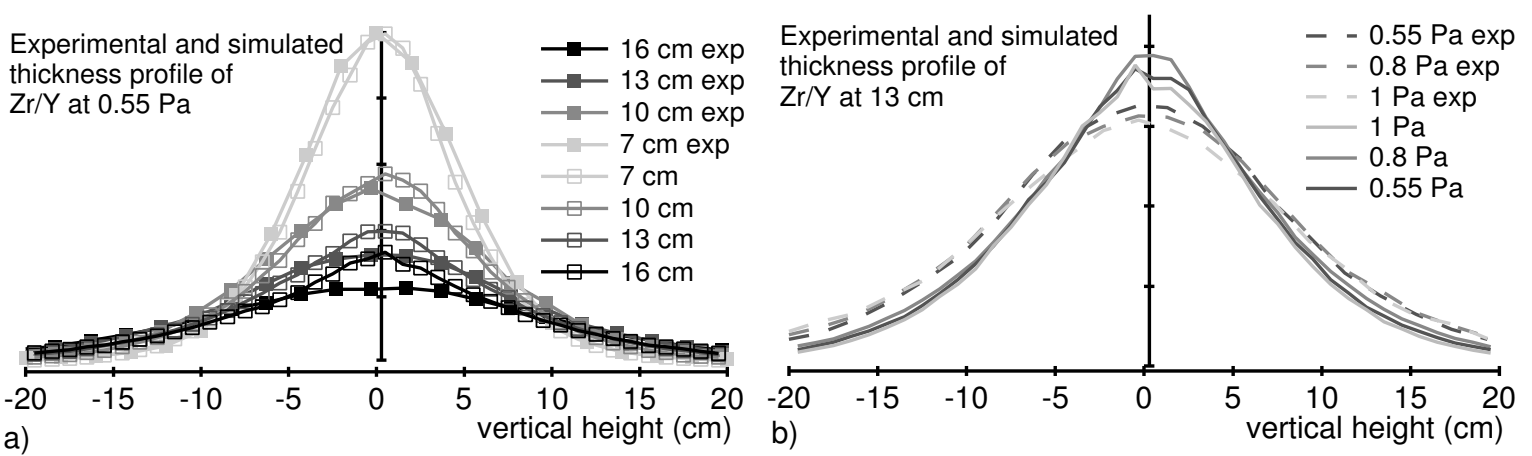

Figure VII.5.4: Simulated and experimental thickness profile of $\mathrm{Zr} / \mathrm{Y}$ as a function of the target-substrate distance (a) and the pressure (b).

For each material ( $\mathrm{Al}, \mathrm{Cu}$ or $\mathrm{Zr} / \mathrm{Y}$ ) one calibration factor has been introduced to be able to compare the experimental and simulated results. This because the experimental data represent a deposition rate (a thickness divided by the deposition time), while the simulations are time independent and only give a thickness profile. More precisely, the simulated data have been multiplied by a factor, in such a way that the simulated and experimental deposition rate at $0.55 \mathrm{~Pa}$, a target-substrate distance of $7 \mathrm{~cm}$ and at a vertical height of $0 \mathrm{~cm}$ are nearly the same. This calibration factor is only necessary to overcome the time independency of the simulation, and has not to be changed in function of the pressure, target-substrate distance or vertical height.

For all materials, the shape of the simulated profiles corresponds very well with the experimentally measured profiles at all pressures and target-substrate distances despite the very large substrate to target size ratio (the correlation coefficient is between 0.9 and 0.99 for e.g. $\mathrm{Cu}$ ). Only in case of $\mathrm{Zr} / \mathrm{Y}$ at larger target-substrate distances, the correspondence between experimental and simulated shape is slightly worse. It is also seen that the influence of the target-substrate distance on the experimental and simulated deposition rate is nearly similar for target-substrate distances in the range between 7 and $13 \mathrm{~cm}$ (the correlation coefficient is 0.98 à 0.99 in case of $\mathrm{Cu}$ ). For all materials the simulated deposition rate at $16 \mathrm{~cm}$ is slightly higher than the experimental deposition rate, but the difference is still acceptable (a correlation coefficient of 0.90 in case of $\mathrm{Cu}$ ). Also the decreasing deposition rate with increasing pressure is seen in the experimental as well as in the simulated deposition rates. Hence, it is concluded that the simulation program is able to reproduce the influence of the pressure and target-substrate distance on the sputtered atom transport for materials which are lighter $(\mathrm{Al})$, heavier $(\mathrm{Cu})$ and much heavier $(\mathrm{Zr}$ and $\mathrm{Y})$ than $\mathrm{Ar}$.

Not only the influence of the target-substrate distance and working pressure on the thickness profile can be simulated, but e.g. also the average energy of the arriving atoms. These 
influences on the average energy of atoms hitting the central square centimeter of the substrate are shown in figure VII.5.5. It is observed that the average energy decreases with target-substrate distance and with pressure, due to an increased number of collisions.

However, it is also seen that heavier atoms ( $\mathrm{Zr}$ and $\mathrm{Y}$ ) hit the substrate with higher energy than lighter atoms. This is merely due to a smaller scattering angle and a less effective energy transfer to the Ar atoms during a collision (see eq.VII.4.8 and VII.4.9).
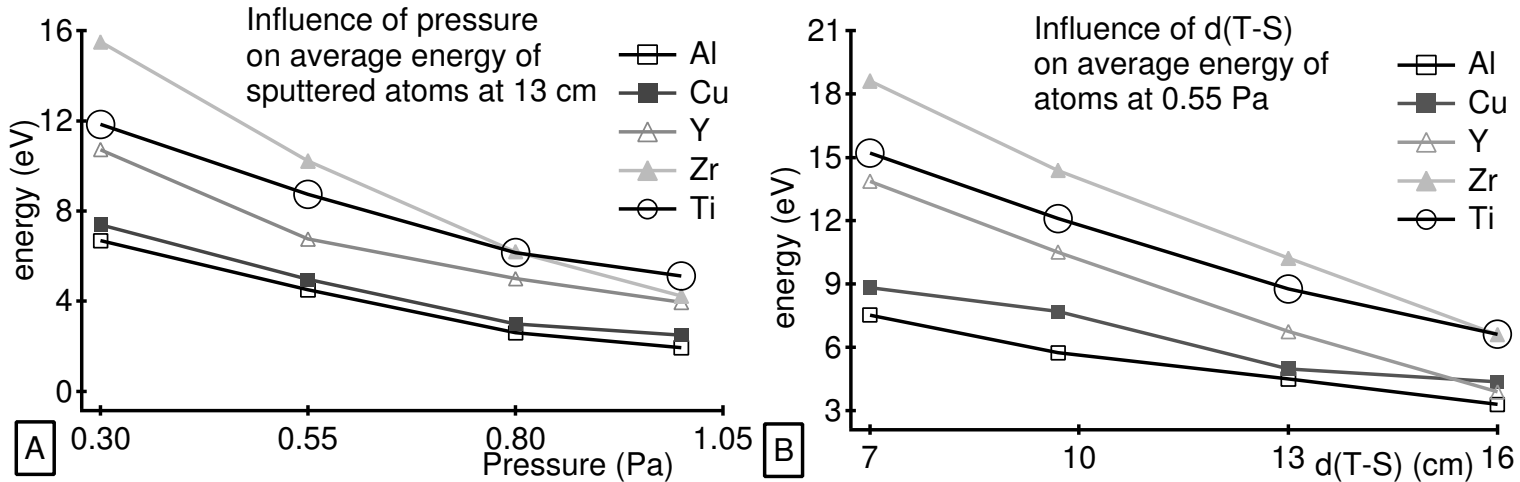

Figure VII.5.5: Influence of the pressure (a) and the target-substrate distance (b) on the average energy of the sputtered particles hitting the central square centimetre of the substrate. The results are shown for $\mathrm{Al}, \mathrm{Cu}, \mathrm{Zr}, \mathrm{Y}$ and Ti.

The average energy of the atoms may seem quite high when compared to the results of other authors [5,31], but it must be noticed that our initial energy distribution of the nascent sputter flux was not cut off at an arbitrary energy. Some atoms with a high energy $(100<\mathrm{E}<400$ $\mathrm{eV}$ ) are sufficient to obtain higher mean energy values.

Although the average energy of the sputtered particles couldn't be measured experimentally, these simulated values indicate that the sputtered particles have a rather high energy when impinging on the growing film. This causes an important contribution to the total energy flux towards the substrate during deposition, as discussed in chapter V (section V.5.3).

\section{VII.5.2: Deposition profile as a function of reactive gas flow:}

The described model (see section VII.4) simulates the nascent sputter flux and the transport of these sputtered particles through the gas phase, for pure metallic depositions. However, all TiN and YSZ films studied in this thesis are deposited by reactive magnetron sputtering. This means that also a reactive gas $\left(\mathrm{O}_{2}\right.$ or $\left.\mathrm{N}_{2}\right)$ was introduced in the chamber during the deposition. Hence the possible influence of the reactive gas should be taken into account.

As discussed in chapter III, at low reactive gas flow, most of the reactive gas will be gettered at the walls (and substrate) and will consequently have a minor influence on the nascent sputter flux or the transport of the metallic particles through the gas phase. This because nearly no reactive gas is present in the chamber, i.e. the partial pressure is nearly zero, and also because the target has not yet much reacted with the reactive gas. However, at higher reactive gas flow, not all the reactive gas will be gettered. This surplus of reactive gas will influence the transport of the particles (collisions with $\mathrm{O}_{2}, \mathrm{~N}_{2}, \ldots$ ) and will also influence the nascent flux because the target gets poisoned. The flow at which the target gets poisoned and the partial pressure of the reactive gas can be monitored by measuring the discharge voltage and the increase in pressure respectively.

In order to check this behaviour, the influence of the reactive gas on the thickness profiles has been investigated for a deposition with a $\mathrm{Zr} / \mathrm{Y}$ 85/15 target. These thickness profiles have been measured experimentally at $0.55 \mathrm{~Pa}$ and $13 \mathrm{~cm}$ with increasing $\mathrm{O}_{2}$-flow $(0,1,2,3$ and 4 $\mathrm{sccm})$. It is observed in figure VII.5.6, that the normalized deposition profile only changes at large $\mathrm{O}_{2}$-flow $(4 \mathrm{sccm})$. The corresponding variation in discharge voltage and pressure due to 
the $\mathrm{O}_{2}$-inlet is shown in figure VII.5.7. Again it is observed that the target only gets poisoned when the reactive flow exceeds $3 \mathrm{sccm}$.

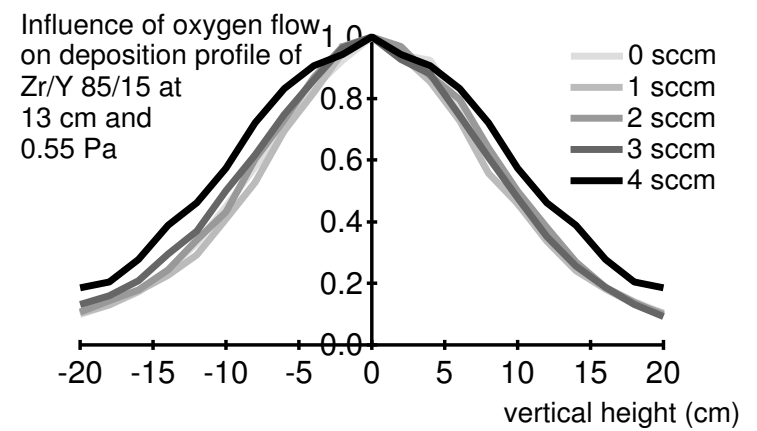

Figure VII.5.6: Normalized experimental thickness profiles of $\mathrm{Zr} / \mathrm{Y}$ 85/15 sputtered in $\mathrm{Ar} / \mathrm{O}_{2}$ mixture at $13 \mathrm{~cm}$ at $0.55 \mathrm{~Pa}$ for $0,1,2,3$ and $4 \mathrm{sccm} \mathrm{O}_{2}$. The profile only changes at $4 \mathrm{sccm}$.

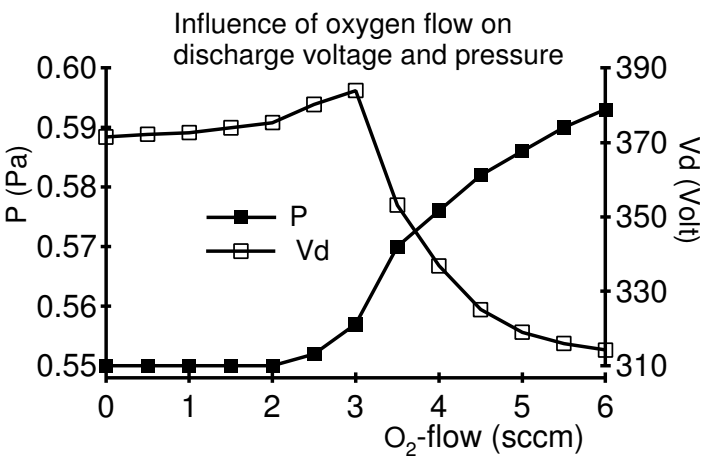

Figure VII.5.7: Variation of the discharge voltage $\mathrm{V}_{\mathrm{d}}$ and the pressure $\mathrm{P}$ due to the increasing $\mathrm{O}_{2}$-flow while sputtering a $\mathrm{Zr} / \mathrm{Y} 85 / 15$ target. The target gets poisoned for an $\mathrm{O}_{2}$-flow exceeding $3 \mathrm{sccm}$.

Hence, in a first approximation, it can be assumed that the nascent sputter flux and the transport of these sputtered particles are not influenced by a reactive gas flow as long as the deposition is not carried out in poisoned mode. Consequently, the described model can also be used to simulate the transport of sputtered particles in reactive magnetron sputtering (but not in poisoned mode). Fortunately, all biaxially aligned thin films were deposited in the nonpoisoned mode. As such, the described model can be used to simulate the influence of several deposition parameters on the angular spread of the material flux towards the substrate.

\section{VII.6. Correlation between in-plane alignment and the deposition conditions}

As concluded in the proposed mechanism for the in-plane alignment (see chapter VI), the resulting in-plane alignment for a deposition carried out in zone $\mathrm{T}$ conditions on a tilted substrate is understood to result from an overgrowth mechanism in which the grains with the largest growth rate overgrow the other grains.

This in-plane growth rate anisotropy was caused by

- the directional diffusion of the adparticles due to the conservation of their momentum when hitting the substrate

- the anisotropy of the capture length for diffusing adparticles as a function of the in-plane orientation due to the growth of the grains according to their kinetically determined habit.

This mechanism of growth rate anisotropy lasts till the out-of-plane alignment is finished, i.e. as long as the $2 \mathrm{D}$ growth can happen. 
Also shadowing can cause an in-plane growth rate anisotropy. This because the former 2D growth rate anisotropy causes the fastest growing grains also to be the tallest. These tallest grains will be the least shadowed and thus will also be favoured to overgrow the other grains.

Both growth rate anisotropies cause the development of an in-plane alignment in an evolutionary way. Therefore, it is logic that the degree of in-plane alignment improves with increasing film thickness, what confirms the experimental results of section VII.2 (figure VII.2.5).

It can be concluded that the degree of in-plane alignment improves with increasing film thickness since the in-plane alignment is caused by an evolutionary overgrowth mechanism. However, it should be mentioned that the XRD technique is not a surface technique, i.e. not the in-plane alignment of the real thin film surface is measured, but the whole film thickness is scanned by the X-rays. As such, the measured influence of the film thickness on the degree of in-plane alignment by XRD as shown in figure VII.2.5, does not reflect the real improvement of the in-plane alignment on the film surface. Therefore, one should make sure that the observed decrease of the FWHM of the XRD poles is not due to a decrease of the relative contribution to the diffraction peaks of the random in-plane oriented grains at the bottom of the film.

The influence of all other deposition parameters on the degree of in-plane alignment was investigated for thin films of the same thickness. As already discussed, the influence of these parameters should be understood as a variation of the mobility during the growth or as a variation in the angular spread on the incoming material flux. First the parameters influencing especially the mobility will be discussed. Then the parameters which also influence the angular spread on the incoming material flux are handled.

- Parameters influencing especially the mobility during the growth.

$\mathrm{Ar} / \mathrm{O}_{2}$ ratio:

Figure VII.2.6. indicates that the in-plane alignment of YSZ films improves when decreasing the $\mathrm{O}_{2}$-flow.

As earlier discussed, growing in zone $\mathrm{T}$ conditions is one of the main conditions to obtain a good in-plane alignment. It was extensively discussed in chapter $\mathrm{V}$ that increasing the $\mathrm{O}_{2}$-flow results in a transition from zone $\mathrm{T}$ conditions to a mixture of zone II and zone T (see V.4). As such, it could be concluded that the lower the $\mathrm{O}_{2-}$ flow during the deposition, the better the [001] out-of-plane alignment and the more the deposition was carried out in perfect zone T conditions. This explains also why the in-plane alignment improves with decreasing $\mathrm{O}_{2}$-flow.

\section{Magnet configuration:}

It was discussed in section V.2 that the in-plane alignment of an YSZ film deposited by an unbalanced magnetron is better than the in-plane alignment of YSZ deposited at similar conditions, but with a balanced magnetron. As already mentioned in chapter III, the use of an unbalanced magnetron causes a high density plasma in front of the substrate. As such, a high electron and ion energy flux towards the substrate sets in, compared to the fluxes corresponding with balanced magnetron sputtering. In this way, it is concluded that the overall mobility during the growth is higher in case of unbalanced magnetron sputtering, compared to balanced sputtering.

This shows that a relative high mobility is needed to obtain good in-plane aligned YSZ films. 
Target usage:

Similarly as with an unbalanced magnetron, the use of an already completely eroded and used target increases the mobility on the growing film, compared to the fluxes during the sputter deposition from a new target. This because the use of an eroded target lowers the metal flux towards the substrate and thus the deposition rate. Lowering the deposition rate and keeping the same electron and ion energy flux results in a higher mobility during the growth. Hence, the mobility during the YSZ deposition from an eroded and used target is higher than during the YSZ deposition from a new target. Again, this higher mobility explains the improved in-plane alignment for an YSZ films deposited from an eroded target, as discussed in section V.2.

\section{Substrate bias:}

As calculated in chapter V (section V.5) the mobility during the deposition can also be varied by applying a bias on the substrate. Applying a negative bias strongly decreases the electron energy flux towards the substrate (see figure V.5.11). As well as for TiN as for YSZ (see figure VII.2.1) a worsening in the in-plane alignment was observed when applying a negative bias. Again, this variation in-plane alignment is understood to be caused by a varied mobility during the growth. A better in-plane alignment is obtained for the situation of a higher electron energy flux towards the substrate, and thus a higher mobility on the growing film.

\section{Chamber walls:}

Also the improved in-plane alignment for a deposition carried out in a chamber with insulating chamber walls can be understood in terms of an increased mobility during the growth. In case of a deposition in a chamber with grounded and metallic chamber walls, the anode ring, the substrate and the chamber walls act as effective anode. However, in case of a deposition in a chamber with insulating walls, only the anode ring and the substrate act as effective anode. This means that the electron current and thus also the electron energy flux towards the substrate is higher in case of the deposition in a chamber with insulating walls, compared to the deposition in a chamber with grounded walls.

It can be concluded that the in-plane alignment of YSZ or TiN improves when carrying out the deposition in as good as possible zone $\mathrm{T}$ conditions (low $\mathrm{O}_{2}$-flow) and by increasing the mobility during the growth (unbalanced magnetron, eroded target, positive substrate bias or insulating chamber walls).

- Parameters also influencing the angular spread of the incoming material flux.

$>$ Target-substrate angle $\alpha$ :

Figure VII.2.2 shows that the in-plane alignment of YSZ and TiN films improves with increasing the target-substrate angle $\alpha$.

An increasing target-substrate angle not only increases the net diffusion distance of an adatom since the diffusion gets more directed when tilting the substrate, but also influences the angular spread on the incoming material flux. That angular spread on the incoming material flux could be calculated by simulating the nascent sputter flux and the collisional transport through the gas phase of the metallic particles (see VII.5). This angular spread as a function of the target-substrate angle has been simulated for the Ti flux towards a substrate at $13 \mathrm{~cm}$ and at a pressure of $0.58 \mathrm{~Pa}$. Also the angular spread for the $\mathrm{Zr} / \mathrm{Y}$ flux towards a substrate at $13 \mathrm{~cm}$ and a pressure of 0.425 has been 
simulated. These calculated angular spreads together with the experimentally observed in-plane alignments are shown in figure VII.6.1.
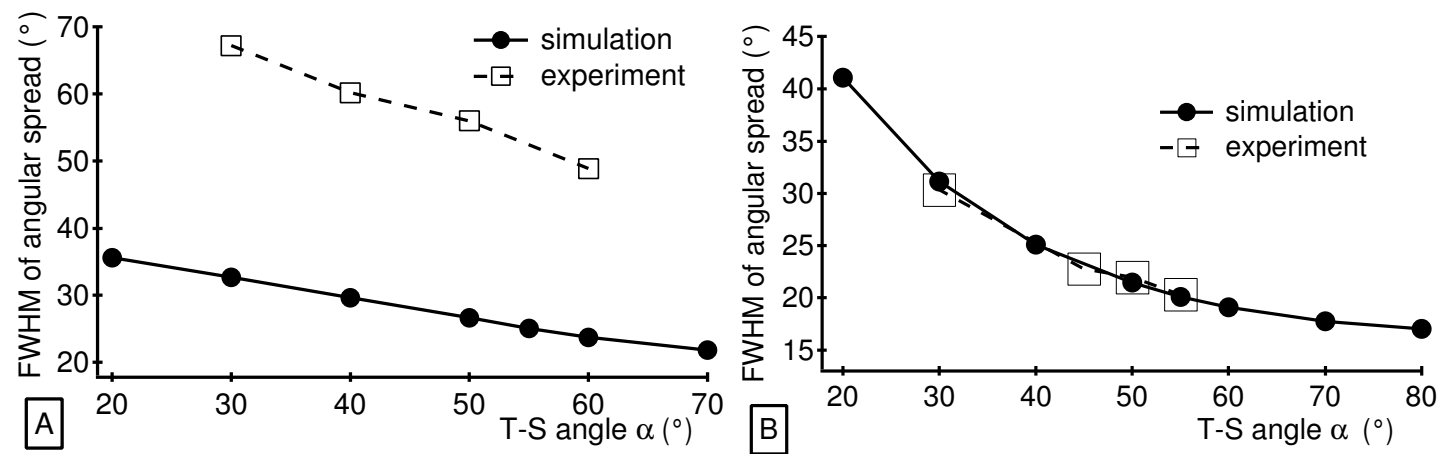

Figure VII.6.1: Influence of the target-substrate angle on the experimental FWHM of the in-plane alignment (open symbols) and the simulated angular spread (closed symbols) for TiN (a) at a pressure of $0.58 \mathrm{~Pa}$ and a target-substrate distance of $13 \mathrm{~cm}$ and for YSZ (b) at a pressure of $0.425 \mathrm{~Pa}$ and a target-substrate distance of $13 \mathrm{~cm}$.

It is observed that the calculated angular spread decreases with increasing targetsubstrate angle $\alpha$ for TiN as well as for YSZ. In case of TiN there is a large difference between the simulated and the experimental values, while for YSZ both experiment and simulation correspond very well. Since the calculated angular spread gives a lower limit for the obtainable in-plane alignment, it must be concluded that the YSZ depositions were carried out at optimal conditions, while in case of TiN the deposition conditions were not at all optimal (with respect to the mobility).

Because the experimentally measured FWHM and the calculated angular spread match nearly perfect in case of YSZ, it can be concluded that the improving in-plane alignment with increasing target-substrate angle $\alpha$ is completely caused by a decreasing angular spread on the material flux.

Target-substrate distance:

Also the influence of the target-substrate distance on the degree of in-plane alignment was investigated. As shown in figure VII.2.4, the in-plane alignment improves with decreasing target-substrate distance as well as for TiN as for YSZ. However, in case of YSZ there is an optimum around $9 \mathrm{~cm}$.

As measured in [32] the energy flux per deposited metallic particle decreases with decreasing target-substrate distance, because the deposition rate increases faster than the energy flux towards the substrate. Consequently, if the in-plane alignment would only depend on the mobility, the degree of in-plane alignment should get worse when lowering the target-substrate distance, because it was already shown that a high mobility is favoured to get a good in-plane aligned YSZ and TiN films.

However, the degree of in-plane alignment is also influenced by the angular spread of the incoming material flux, which also strongly depends on the target-substrate distance. This influence of the target-substrate distance on the angular spread and on the experimental degree of in-plane alignment is shown in figure VII.6.2 for TiN and YSZ.

It is observed that the angular spread of the incoming material flux continuously decreases with decreasing target-substrate distance in case of TiN. However, in case of YSZ, the angular spread on the $\mathrm{Zr} / \mathrm{Y}$ flux shows a minimum at $9 \mathrm{~cm}$, perfectly corresponding with the observed minimum in the degree of in-plane alignment. 
As such, it can be concluded that the tendency of the simulated angular spread as a function of the target-substrate distance completely agrees with the observed tendency in the degree of in-plane alignment, as well as for TiN as for YSZ.

Again, it is observed that the experimental values for TiN are much worse than the simulated ones, indicating that the deposition conditions were once more not optimal. Finally, it is also observed that the experimental FWHM values correspond very well with the simulated angular spread for YSZ, at least for a target-substrate distance of at least $9 \mathrm{~cm}$. In case of a very small target-substrate distance, i.e. $7 \mathrm{~cm}$, the adparticle mobility is probably too low [32], causing a worse in-plane alignment than the calculated angular spread.
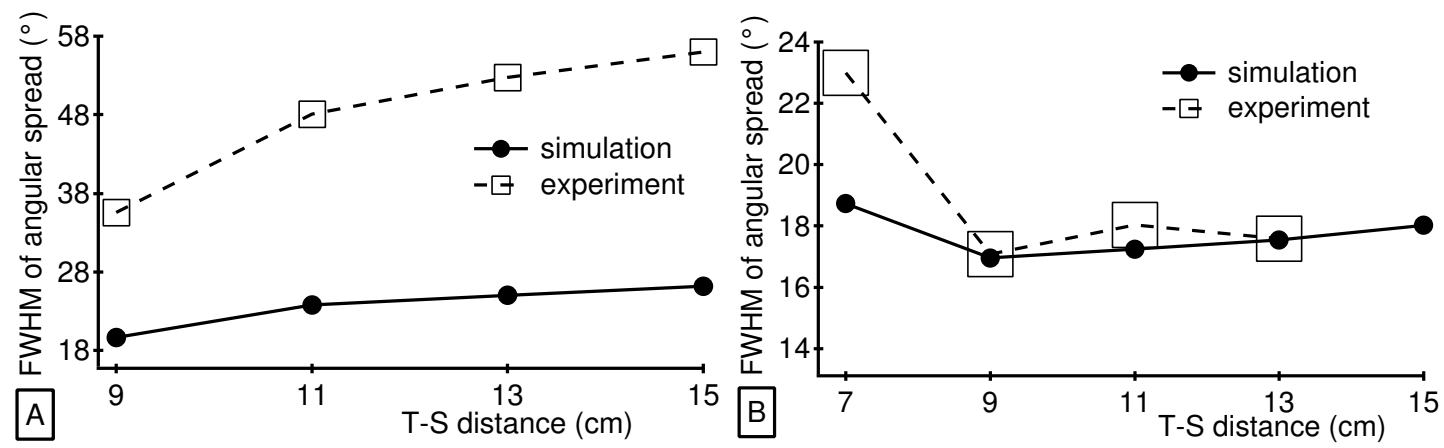

Figure VII.6.2: Influence of the target-substrate distance on the experimental FWHM of the in-plane alignment (open symbols) and the simulated angular spread (closed symbols) for TiN (a) at a pressure of $0.58 \mathrm{~Pa}$ and a target-substrate angle of $55^{\circ}$ and for YSZ (b) at a pressure of $0.425 \mathrm{~Pa}$ and a targetsubstrate angle of $55^{\circ}$.

\section{Pressure:}

Finally also the influence of the pressure could be understood in terms of the mobility and the angular spread on the incoming material flux. As shown in figure VII.2.3, the in-plane alignment of YSZ and TiN first improve with decreasing pressure, but then at too low pressures- the in-plane alignment get worse again.

It was measured in [32-35] that the energy flux per deposited particle, and thus also the mobility, decreases with decreasing pressure. Again, if the in-plane alignment would only depend on the mobility, the FWHM should get worse with decreasing pressure.
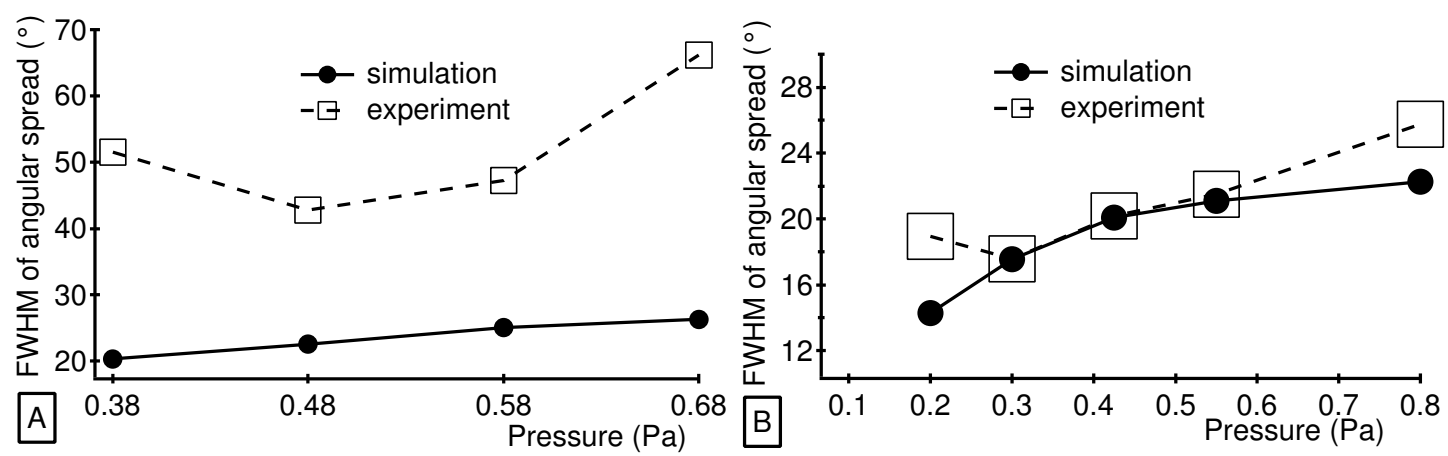

Figure VII.6.3: Influence of the pressure on the experimental FWHM of the in-plane alignment (open symbols) and the simulated angular spread (closed symbols) for TiN (a) at a target-substrate distance of $13 \mathrm{~cm}$ and a target-substrate angle of $55^{\circ}$ and for YSZ (b) at a target-substrate distance of $13 \mathrm{~cm}$ and a target-substrate angle of $55^{\circ}$.

Beside the mobility, also the angular spread is influenced by the pressure. As shown in figure VII.6.3, the angular spread continuously decreases with decreasing pressure. 
As such, it can be concluded that the in-plane alignment first improves with decreasing pressure due to a decreasing angular spread. But then get worse due to a too low mobility during the deposition [32-35].

Again, it is observed that the calculated angular spread is much better than the obtained FWHM for the TiN films, indicating that the deposition conditions still had to be optimized. For YSZ, the calculated values and the experimental values correspond very well in the region between 0.3 and $0.8 \mathrm{~Pa}$.

After optimizing all parameters like substrate bias, reactive gas flow, film thickness, ... an inplane alignment of $18.1^{\circ}+/-0.9^{\circ}$ in FWHM could be obtained for a TiN deposition on a $+15 \mathrm{~V}$ biased polycrystalline stainless steel substrate, a target-substrate distance of $9 \mathrm{~cm}$, an $\mathrm{Ar} / \mathrm{N}_{2}$ ratio of 55/5, a target-substrate angle of $60^{\circ}$, a pressure of $0.48 \mathrm{~Pa}$ and a film thickness of 1.7 $\mu \mathrm{m}$. The simulated angular spread of the incoming Ti-flux at these conditions (tilt angle of $60^{\circ}$, distance of $9 \mathrm{~cm}$ and pressure of $0.48 \mathrm{~Pa}$ ) is $18.2^{\circ}+/-0.5^{\circ}$ in FWHM, what exactly corresponds with the experimental observed in-plane alignment.

In case of YSZ, an in-plane alignment of $14.8^{\circ}+/-0.7^{\circ}$ could be obtained at the optimized deposition conditions: a $+15 \mathrm{~V}$ biased substrate, a film thickness of $1.3 \mu \mathrm{m}$, a target-substrate distance of $9 \mathrm{~cm}$, a target-substrate angle of $65^{\circ}$, a pressure of $0.3 \mathrm{~Pa}$ and an $\mathrm{Ar} / \mathrm{O}_{2}$ ratio of $58 / 1.7 \mathrm{sccm}$. Calculation of the angular $\mathrm{Zr} / \mathrm{Y}$ spread at these conditions resulted in a spread of $15.4^{\circ}+/-0.5^{\circ}$, what again corresponds well with the experimental results.

\section{VII.7. Conclusions}

Although it was observed that a lot of deposition parameters such as substrate bias, targetsubstrate distance, angle, pressure, thickness, reactive gas flow, target usage, magnet configuration, condition of the chamber walls influence the degree of in-plane alignment, their influence could be reduced to two fundamental parameters, i.e. the mobility during the growth and the angular spread of the incoming material flux.

It was shown that the adparticle mobility should be high enough to allow the full completion of the 2D growth, i.e. the adparticle must be able to reach the edge of a "good" out-of-plane oriented grain. However, that adparticle mobility can't be too high, since the deposition has to be carried out in perfect zone T conditions, i.e. no re-structurative grain growth is allowed. Beside that mobility, it was shown that the angular spread of the incoming material flux imposes a lower limit on the obtainable degree of in-plane alignment. That angular spread has been calculated by simulating the nascent sputter flux and the transport through the gas phase of the sputtered particles by a binary collision Monte Carlo code. As such, a quantitative value of that lower limit was obtained.

Optimizing all deposition parameters for TiN and YSZ, it was shown that the obtained experimental degree of in-plane alignment corresponds very well with the calculated lower limit, i.e. the angular spread.

To be able to completely understand the developed in-plane alignment, also a quantitative value for the adparticle mobility as a function of the used deposition parameters should be available. However, this is not straightforward and should be the scope of further basic research.

\section{References}

1: G. De Winter: Ph.D thesis: "Studie van de sputter depositie van Yttria gestabiliseerd zirconia bufferlagen voor coated conductors" UGent 2004.

2: C. Eisenmenger-Sittner, R. Beyerknecht, A. Bergauer, W. Bauer, G. Betz: J. Vac. Sci. Technol. A 13(5) (1995) p.2435-2443

3: G.M. Turner, S.M. Rossnagel, J.J. Cuomo, J. Appl. Phys. 75(7) (1994) p.3611-3618 
4: W.D. Westwood: J. Vac. Sci. Technol. 15(1) (1978) p.1-9

5: T. Motohiro, Y. Taga: Surface Science Letters 134 (1) (1983) L494-L499

6:G.M. Turner, I.S. Falconer, B.W.James, D.R. McKenzie: J. Appl. Phys. 65(9) (1989) p.3671-3679

7: V. Abhilash, R. Balu, S. Balaji, S. Sentil Nathan, S. Mohan: Computational Material Science 30 (2004) p.523-529

8: K. Maçak, P. Macak, U. Helmersson: Computer Physics Communications 120 (1999) p. $238-254$

9: S. Sentil Nathan, G. Mohan Rao, S. Mohan: J. Appl. Phys. 84(1) (1998) p.564-570

10:Y. Taga, R. Takahasi: Surface Science 386 (1997) p.231-240

11: A. Kersch, W. Morokoff, Chr. Werner: J. Appl. Phys. 75(4) (1994) p.2278-2284

12: C. Eisenmenger-Sittner, R. Beyerknecht, A. Bergauer: J. Vac. Sci. Technol. A13(5) (1995) p.2435-2443

13: J.C.S. Kools: J. Appl. Phys, 74(10) (1993) p.6401-6406

14:G.M. Turner, I.S. Falconer, B.W. James, D.R. McKenzie: J. Vac. Sci. Technol. A10 (3) (1992) p.455-461

15: K. Deenamma Vargheese, G. Mohan Rao: J. Appl. Phys. 87(10) (2000) p.7544-7550

16: P.K. Petrov, V.A. Volpyas, R.A. Chakalov: Vacuum 52 (1999) p.427-434

17:V.V. Serikov, K. Nanbu: J. Vac. Sci. Technol. A14(6) (1996) p.3108-3123

18: T. Smy, L. Tan, S.S. Winterton, S.K. Dew, M.J. Brett: J. Vac. Sci. Technol. A15(6) (1997) p.2847-2853

19: S.M. Rossnagel, J. Vac. Technol. A 21(5) (2003) S74-S87

20: A.M. Myers, J.R. Doyle, J.R. Abelson, D.N. Ruzic: J. Vac. Sci. Technol. A 9(3) (1991) p.614-618

21: Y. Yamamura, M. Ishida: J. Vac. Sci. Technol. A(13)1 (1995) p.101-112

22: A.M. Myers, J.R. Doyle, D.N. Ruzic: J. Appl. Phys. 72(7) (1992) p.3064-3071

23: Stopping and Range of Ions in Matter: www.srim.org

24: W. Eckstein in "Computer simulation of ion-solid interactions" published by Springer-

Verlag New York Berlin Heidelberg, May 1991 ISBN: 0-687-19057-0

25: S.M. Rossnagel: IEEE T. Appl. Plasma Sci. 18(6) (1990) p.878-882

26: A. Palermo, H. Rudolph, F.H.P.M. Habraken: Appl. Phys. Lett. (87) (2005) p.071501

27: B. Chapman in "Glow Discharge Processes" published by John Wiley \& sons ISBN: 0 $47107828 \mathrm{X}$

28: D.E. Harrison, C.B. Delaplain: J. Appl. Phys. 47(6) (1976) p.2252-2259

29: E.W. McDaniel in "Collision Phenomena in Ionized Gases" published by Wiley New York (1964) ASIN: B0006BLWZA

30: R.S. Robinson: J. Vac. Sci. Technol. A16(2) (1979) p.185- 188

31: K. Meyer, I.K. Schuller, C.M.M. Falco : J. Appl. Phys. 52(9) (1981) p.5803-5805

32: S.D. Ekpe, S.K. Dew: J. Vac. Sci. Technol. A 22(4) (2004) p.1420-1424

33: T.P. Drüsedau, T. Bock, T.M. John, F. Klabunde, W. Eckstein: J. Vac. Sci. Technol. A 17(5) (1999) p.2896-2905

34: S. D. Ekpe, S.K. Dew: J. Vac. Sci. Technol. A 21(2) (2003) p.476-483

35: S. D. Ekpe, S.K. Dew: J. Vac. Sci. Technol. A 20(6) (2002) p.1877-1885 


\section{Appendix:}

Code Transport of non-thermalized particles:

Option Explicit

Public A As Single

Public B As Single

Public C As Single

Public D As Single

Public E As Single

Public F As Double

Public G As Double

Public H As Double

Public P As Single

Public R As Single

Public T As Single

Public een As Double

Public twee As Double

Public drie As Double

Public vier As Double

Public x As Double

Public y As Double

Public z As Double

Public afstand As Double

Public aantalbots As Single

Public aantaltherm As Single

Public aantaleind As Single

Public aantalwand As Single

Public bestand As Single

Public bmax As Single

Public buffer As String

Public Elab As Double

Public Ecom As Single

Public Ecom1 As Single

Public Ecom2 As Single

Public Etherm As Single

Public Even As Single

Public extraR As Single

Public halt As Single

Public keuze As Single

Public landa As Double

Public Ndeeltjes As Single

Public Phivoor As Double

Public Phiextra As Double

Public Phisamen As Double

Public rij As Single

Public rij2 As Single

Public teller As Single

Public teller_r As Single

Public teller_k As Single

Public teller_1 As Single

Public thetavoor As Double

Public thetaextra As Double

Public thetasamen As Double

Public thetacom As Double

Public druk As Single

Public massa As Single

Public straal As Single

Public mverh As Single

Public test As Double

Public test1 As Double

Public test2 As Double
Public test3 As Single

Public aantaltotaal As Single

Public racetrack As Single

Public TSafstand As Single

Public v_xy As Double

Public v_xyz As Double

Public v_x As Double

Public v_y As Double

Public v_z As Double

Public costhetavoor As Double

Public sinthetavoor As Double

Public cosphivoor As Double

Public sinphivoor As Double

Private Const csttotaalaantalmax $=2000000$

Private Const tijdstap $=0.0000001$

Private Const conpi $=3.14159265358979$

Private matrixtherm(csttotaalaantalmax, 6) As Single

Private matrixeind(csttotaalaantalmax, 6) As Single

Private matrixwand(csttotaalaantalmax, 6) As Single

Private matrixthetacom $(477,200)$ As Single

Private matrixthetalab(3136) As Single

Private startersset $(200000,1)$ As Double

Private matrixstartplaats(600) As Single

Private Sub Cmdstart_Click()

Randomize

'reading the file where thetacoms were calculated

bestand $=$ FreeFile

buffer = "D:\mijn documenten $\backslash$ Stijn $\backslash$ sim richting atomen $\backslash$ thetacom

berekenen/matricestotaal lthetacomTi.csv"

Open buffer For Input As bestand

For teller_r $=0$ To 477

For teller_k $=0$ To 200

Input \#bestand, matrixthetacom(teller_r, teller_k)

Next teller_k

Input \#bestand, buffer

Next teller_r

Close bestand

'reading the file where thetalab was calculated bestand $=$ FreeFile

buffer = "D:|mijn documenten $\backslash$ Stijn $\backslash$ sim richting

atomen $\backslash$ thetalab berekenen $\backslash$ berekende

thetalabmatrices $\backslash$ thetalabTi.dat"

Open buffer For Input As bestand

For teller_r $=0$ To 3136

Input \#bestand, matrixthetalab(teller_r)

Next teller_r

Close bestand

'reading the file where start place $\mathrm{R}$ was calculated

bestand $=$ FreeFile

buffer = "D: \mijn documenten $\backslash$ Stijn $\backslash$ sim richting

atomenlstartersset

berekenen|startersplaats \startplaats2.dat"

Open buffer For Input As bestand

For teller_r $=0$ To 600

Input \#bestand, matrixstartplaats(teller_r)

Next teller_r 
Close bestand

'reading the file where start set was calculated bestand $=$ FreeFile

buffer = "D: \mijn documenten $\backslash$ Stijn $\backslash$ sim richting atomenlstartersset

berekenen|starterssets\starterssetTi.csv"

Open buffer For Input As bestand

For teller_r $=0$ To 200000

For teller_k $=0$ To 1

Input \#bestand, startersset(teller_r, teller_k)

Next teller_k

Input \#bestand, buffer

Next teller_r

Close bestand

'writing initializing values in text box

gegevens. Text $=$ txtdruk \& $" \mathrm{~Pa} / " \&$

txtstraalracetrack \& " cm/" \& txtmassa \& " a.m.e./"

\& txtstraal \& "angstr./" \& txtTSafstand \& " cm/" \& txtaantaltotaalmax \& "maximaal aantal deeltjes"

'counter of particles set on 0

druk $=$ Val(txtdruk)

straal $=$ Val (txtstraal $)$

massa $=$ Val (txtmassa $)$

aantaltotaal $=\mathrm{Val}($ txtaantaltotaalmax $)$

racetrack $=\mathrm{Val}($ txtstraalracetrack $)$

TSafstand $=$ Val $($ txtTSafstand $)$

Ndeeltjes $=-1$

aantaltherm $=0$

aantalwand $=0$

aantaleind $=0$

redeposition $=0$

'this is value where particles are treated to be

thermalized

Etherm $=0.03$

'let it run until the number of particles is equal to aantaltotaalmax

While Ndeeltjes < (aantaltotaal - 1)

'choose a random sputtered particle from start set

keuze $=\operatorname{Int}($ Rnd $* 200001)$

thetavoor $=$ startersset $($ keuze, 1$)$

Randomize

$\mathrm{F}=\left(\mathrm{Rnd}^{\wedge} 1.05\right)$

test $=\operatorname{Atn}(\mathrm{F} / \operatorname{Sqr}(-\mathrm{F} * \mathrm{~F}+1))$

If test $<=$ thetavoor Then

Phiextra $=0$

thetaextra $=0$

Elab $=$ startersset $($ keuze, 0$)$

'count the number of particles and stop loop if necessary

Randomize

Phivoor $=\left(\operatorname{Rnd}^{\wedge} 1.05\right) *$ conpi $* 2$

teller_l $=0$

Randomize

$\mathrm{D}=\mathrm{Rnd}^{\wedge} 1.05$

Do

teller_ $1=$ teller_ $1+1$

Loop Until matrixstartplaats(teller_l) $>=$ D

extraR $=(($ teller_l $* 0.01)-3)$

$\mathrm{R}=$ racetrack + extraR
Randomize

$\mathrm{E}=\left(\mathrm{Rnd}^{\wedge} 1.05\right) * 2 *$ conpi

$\mathrm{x}=\mathrm{R} * \operatorname{Cos}(\mathrm{E})$

$\mathrm{y}=\mathrm{R} * \operatorname{Sin}(\mathrm{E})$

$\mathrm{z}=0$

$\mathrm{v} \_\mathrm{x}=\operatorname{Sin}($ thetavoor $) * \operatorname{Cos}($ Phivoor $)$

$\mathrm{v} \_\mathrm{y}=\operatorname{Sin}($ Phivoor $) * \operatorname{Sin}($ thetavoor $)$

$\mathrm{v} \_\mathrm{z}=\operatorname{Cos}($ thetavoor $)$

$\mathrm{v} \_\mathrm{xy}=\operatorname{Sqr}\left(\mathrm{v} \_\mathrm{x} * \mathrm{v} \_\mathrm{x}+\mathrm{v} \_\mathrm{y} * \mathrm{v} \_\mathrm{y}\right)$

$\mathrm{v} \_x y z=\operatorname{Sqr}\left(\mathrm{v} \_z * v \_z+\mathrm{v} \_x y * v \_x y\right)$

costhetavoor $=\mathrm{v} \_\mathrm{z} / \mathrm{v} \_\mathrm{xyz}$

sinthetavoor $=\mathrm{v} \_\mathrm{xy} / \mathrm{v} \_\mathrm{xyz}$

Ndeeltjes $=$ Ndeeltjes +1

aantalbots $=0$

halt $=0$

'all start values of the Nth particle are determined

'let the particle run and collide untill halt $=1$

Do While halt $=0$

Randomize

'calculate Ecom

Ecom $=($ Elab $* 39.948 /$ massa $) /(1+39.948 /$

massa)

$\mathrm{C}=$ tijdstap

'let the particle run

'first calculate the distance that the particle runs in one timestep

afstand $=100 *\left(\left(\left(2 *\right.\right.\right.$ Elab $\left.* 1.602 * 10^{\wedge}(-19)\right)$

$\left.\left./\left(\operatorname{massa} * 1.66 * 10^{\wedge}(-27)\right)\right)^{\wedge} 0.5\right) * \mathrm{C}$

'procedure in which we store the thermalized

particles

If Ecom < Etherm Then

If $v \_z>=1$ Then

thetasamen $=0$

ElseIf $\mathrm{v} \_\mathrm{z}<=-1$ Then

thetasamen $=$ conpi

Else

thetasamen $=\operatorname{Atn}\left(-\mathrm{v} \_\mathrm{z} / \operatorname{Sqr}\left(-\mathrm{v} \_\mathrm{z} * \mathrm{v} \_\mathrm{z}+\right.\right.$

1)) $+2 * \operatorname{Atn}(1)$

End If

vier $=\operatorname{Sin}($ thetasamen $)$

If vier $=0$ Then

Phisamen $=0$

ElseIf ( $v \_x /$ vier $)>=1$ Then

Phisamen $=0$

ElseIf (v_x / vier) $<=-1$ Then

Phisamen $=$ conpi

Else

Phisamen $=$ Atn $\left(-\left(v_{-}\right.\right.$x $/$vier $) / \operatorname{Sqr}\left(-\left(v_{-}\right.\right.$x $/$

vier $) *\left(v_{-} \mathrm{x} /\right.$ vier $\left.\left.)+1\right)\right)+2 * \operatorname{Atn}(1)$

End If

If vier $=0$ Then

Phisamen $=0$

ElseIf (v_y / vier) < 0 Then

Phisamen $=-$ Phisamen

End If

matrixtherm $($ aantaltherm, 0$)=x$

matrixtherm $($ aantaltherm, 1$)=y$

matrixtherm(aantaltherm, 2$)=\mathrm{z}$

matrixtherm (aantaltherm, 3) $=$ thetasamen

matrixtherm $($ aantaltherm, 4$)=$ Phisamen 
matrixtherm(aantaltherm, 5) = Elab matrixtherm(aantaltherm, 6) = aantalbots aantaltherm $=$ aantaltherm +1 halt $=1$

'procedure in which we store the particles which reached the substrate

ElseIf $\mathrm{z}>=$ TSafstand Then

If $\mathrm{v} \_\mathrm{z}>=1$ Then

thetasamen $=0$

ElseIf $\mathrm{v} \_\mathrm{z}<=-1$ Then

thetasamen $=$ conpi

Else

thetasamen $=$ Atn $\left(-\mathrm{v} \_\mathrm{z} / \operatorname{Sqr}\left(-\mathrm{v} \_\mathrm{z} * \mathrm{v} \_\mathrm{z}\right.\right.$

$+1))+2 * \operatorname{Atn}(1)$

End If

vier $=\operatorname{Sin}($ thetasamen $)$

If vier $=0$ Then

Phisamen $=0$

ElseIf (v_x / vier) $>=1$ Then

Phisamen $=0$

ElseIf (v_x / vier) $<=-1$ Then

Phisamen $=$ conpi

Else

Phisamen $=\operatorname{Atn}\left(-\left(\mathrm{v} \_\mathrm{x} /\right.\right.$ vier $) / \operatorname{Sqr}(-$ $\left(\mathrm{v} \_\mathrm{x} /\right.$ vier $\left.\left.) *\left(\mathrm{v} \_\mathrm{x} / \mathrm{vier}\right)+1\right)\right)+2 *$

$\operatorname{Atn}(1)$

End If

If vier $=0$ Then

Phisamen $=0$

ElseIf (v_y / vier) $<0$ Then

Phisamen $=-$ Phisamen

End If

matrixeind (aantaleind, 0$)=\mathrm{x}$

matrixeind (aantaleind, 1$)=y$

matrixeind(aantaleind, 2 ) $=\mathrm{z}$

matrixeind(aantaleind, 3$)=$ thetasamen

matrixeind (aantaleind, 4$)=$ Phisamen

matrixeind (aantaleind, 5) $=$ Elab

matrixeind (aantaleind, 6 ) $=$ aantalbots

aantaleind $=$ aantaleind +1

halt $=1$

'procedure to store the particles which reach the chamber walls

ElseIf $\mathrm{z}<0$ Or $\operatorname{Abs}(\mathrm{x})>25 \operatorname{Or} \operatorname{Abs}(\mathrm{y})>25$

Then

matrixwand (aantalwand, 0$)=x$

matrixwand (aantalwand, 1$)=y$

matrixwand (aantalwand, 2$)=\mathrm{z}$

matrixwand(aantalwand, 3$)=0$

matrixwand (aantalwand, 4$)=0$

matrixwand (aantalwand, 5) $=$ Elab

matrixwand(aantalwand, 6 ) $=$ aantalbots

aantalwand $=$ aantalwand +1

halt $=1$

Else

'check if the particles collide or not and thus first calculate the mean free path Select Case Elab

Case Is $<=1$

$$
\begin{aligned}
& \text { landa }=0.13178 /(\text { druk } *((1+(\text { massa } / \\
& \left.\left.39.948))^{\wedge} 0.5\right) *\left((\text { straal }+0.102)^{\wedge} 2\right)\right) \\
& \text { Case Is }>1 \\
& \text { landa }=0.13178 *\left(\text { Elab }^{\wedge} 0.29\right) /(\text { druk } * \\
& \left((1+(\text { massa } / 39.948))^{\wedge} 0.5\right) *((\text { straal }+ \\
& \left.\left.0.102)^{\wedge} 2\right)\right)
\end{aligned}
$$

End Select

$\mathrm{P}=1$ - Exp(-afstand / landa)

If $\mathrm{P}>\left(\mathrm{Rnd}^{\wedge} 1.05\right)$ Then

'if bots $=1$ the particle collides: re-calculate $E$ and angle

aantalbots $=$ aantalbots +1

'select the thetalab corresponding with this

Ecom

Select Case Ecom

Case Is $<1$

Ecom $1=(\operatorname{Int}(E \operatorname{com} * 100) / 100)$

Ecom $2=(\operatorname{Int}($ Ecom $* 1000) / 1000)$

If $($ Ecom $2-$ Ecom 1$)<0.005$ Then

$\mathrm{rij}=((\mathrm{Ecom} 1-0.03) * 100)$

Else

rij $=(($ Ecom $1-0.03) * 100)+1$

End If

Case Is $<10$

Ecom $1=(\operatorname{Int}($ Ecom $* 10) / 10)$

Ecom $2=(\operatorname{Int}($ Ecom $* 100) / 100)$

If $($ Ecom $2-$ Ecom 1$)<0.05$ Then

rij $=(((E c o m 1-1) * 10)+97)$

Else

rij $=((($ Ecom1 -1$) * 10)+97)+1$

End If

Case Is $<300$

Ecom1 $=\operatorname{Int}($ Ecom $)$

Ecom $2=(\operatorname{Int}($ Ecom $* 10) / 10)$

If $($ Ecom2 - Ecom 1$)<0.5$ Then

$\mathrm{rij}=(($ Ecom $1-10)+187)$

Else

$\mathrm{rij}=(($ Ecom $1-10)+187)+1$

End If

Case Is $>=300$

Ecom1 $=300$

rij $=477$

End Select

'choose $b$, thus first read bmax

teller = matrixthetacom(rij, 200)

Randomize

Do

$\mathrm{B}=\operatorname{Int}\left((\right.$ teller +1$\left.) *\left(\left(\operatorname{Rnd}^{\wedge} 1.05\right)^{\wedge} 0.5\right)\right)$

'read the matrixnumber corresponding with

$\mathrm{b}$ and Ecom

Randomize

Select Case B

Case Is $=0$

thetacom $=$ conpi

Case Is $>0$

thetacom $=$ matrixthetacom $($ rij, $(B-1))$

End Select

$\mathrm{G}=$ Rnd $^{\wedge} 1.05$

If thetacom < (conpi / 2) Then

$\mathrm{H}=$ thetacom 


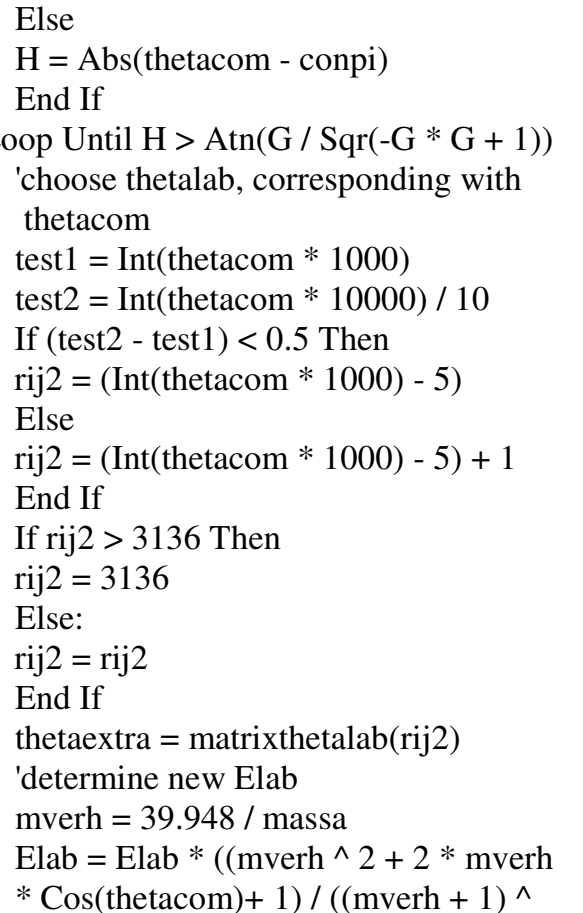

2))

Randomize

Phiextra $=$ Rnd $*$ conpi $* 2$

'let the particle run in the new direction

$\mathrm{x}=\mathrm{x}+$ afstand $* \mathrm{v} \_\mathrm{x}$

$\mathrm{y}=\mathrm{y}+$ afstand $*$ v_y

$\mathrm{z}=\mathrm{z}+$ afstand $* \mathrm{v} \_\mathrm{z}$

$\mathrm{v} \_\mathrm{xy}=\operatorname{Sqr}\left(\mathrm{v} \_\mathrm{x} * \mathrm{v} \_\mathrm{x}+\mathrm{v} \_\mathrm{y} * \mathrm{v} \_\mathrm{y}\right)$

$\mathrm{v} \_x y z=\operatorname{Sqr}\left(\mathrm{v} \_z * \mathrm{v} \_z+\mathrm{v} \_x y * v \_x y\right)$

costhetavoor $=\mathrm{v} \_z / \mathrm{v} \_\mathrm{xyz}$

sinthetavoor $=v \_x y / v \_x y z$

If sinthetavoor $=0$ Then

cosphivoor $=1$

sinphivoor $=0$

Else

cosphivoor $=\mathrm{v} \_\mathrm{x} /$ sinthetavoor

sinphivoor $=\mathrm{v} \_\mathrm{y} /$ sinthetavoor

End If

$\mathrm{v} \_\mathrm{x}=\cos$ thetavoor $* \operatorname{cosphivoor} *$

$\operatorname{Sin}($ thetaextra) $* \operatorname{Cos}($ Phiextra $)-$ sinphivoor $*$

$\operatorname{Sin}($ thetaextra $) * \operatorname{Sin}($ Phiextra $)+\sin$ thetavoor $*$

cosphivoor $* \operatorname{Cos}($ thetaextra)

v_y $=\cos$ thetavoor $* \operatorname{sinphivoor} *$

$\operatorname{Sin}($ thetaextra $) * \operatorname{Cos}($ Phiextra $)+\operatorname{cosphivoor} *$

$\operatorname{Sin}($ thetaextra $) * \operatorname{Sin}($ Phiextra $)+$ sinphivoor $*$

sinthetavoor $* \operatorname{Cos}($ thetaextra $)$

$\mathrm{V} \_\mathrm{z}=\cos$ thetavoor $* \operatorname{Cos}($ thetaextra $)$ -

sinthetavoor $* \operatorname{Sin}($ thetaextra $) * \operatorname{Cos}($ Phiextra $)$

Else

'just let the particle run

$\mathrm{x}=\mathrm{x}+$ afstand $* \mathrm{v} \_\mathrm{x}$

$\mathrm{y}=\mathrm{y}+$ afstand $*$ v_y

$\mathrm{z}=\mathrm{z}+$ afstand $* \mathrm{v} \_\mathrm{z}$

End If

' this was end of procdure for one collision End If

' this was end of loop to follow a particle one timestep

Loop

'this is end of collideloop because particle reached the walls, substrate or get thermalized

End If

'this is endif of control sinhoek $<$ rnd

Wend

'this is end of loop because aantaltotaalmax particle is reached

'the three procedure to store the particles in the right

files.

bestand $=$ FreeFile

Open txtmateriaal \& "_" \& txtlabel \& "_" \& txtdruk \&

"_" \& txtTSafstand \& "_" \& "therm" \& ".csv" For

Output As bestand

buffer $=$ " "

For teller_r $=0$ To $($ aantaltherm - 1)

For teller_k $=0$ To 6 buffer $=$ buffer $\&$ matrixtherm(teller_r, teller_k) \& ","

Next teller_k

Print \#bestand, buffer

buffer $="$ "

Next teller_r

Close bestand

bestand $=$ FreeFile

Open txtmateriaal \& "_" \& txtlabel \& "_" \& txtdruk \&

"_" \& txtTSafstand \& "_" \& "wand" \& ".csv" For

Output As bestand

buffer = " "

For teller_r $=0$ To (aantalwand - 1)

For teller_k $=0$ To 6

buffer $=$ buffer $\&$ matrixwand(teller_r, teller_k) $\&$ ","

Next teller_k

Print \#bestand, buffer

buffer $="$ "

Next teller_r

Close bestand

bestand $=$ FreeFile

Open txtmateriaal \& "_" \& txtlabel \& "_" \& txtdruk \&

"_" \& txtTSafstand \& "_" \& "eind" \& ".csv" For Output

As bestand

buffer $="$ "

For teller_r $=0$ To (aantaleind - 1)

For teller_k $=0$ To 6 buffer $=$ buffer $\&$ matrixeind(teller_r, teller_k) $\&$ ","

Next teller_k

Print \#bestand, buffer

buffer $=$ " "

Next teller_r

Close bestand

txtaantaleind.Text $=$ aantaleind

txtaantalwand.Text $=$ aantalwand

txtaantaltherm. Text $=$ aantaltherm

bestand $=$ FreeFile

Open txtmateriaal \& "_" \& txtlabel \& "_" \& txtdruk \& "_" \& txtTSafstand \& "_" \& "geg" \& ".txt" For Output As bestand 


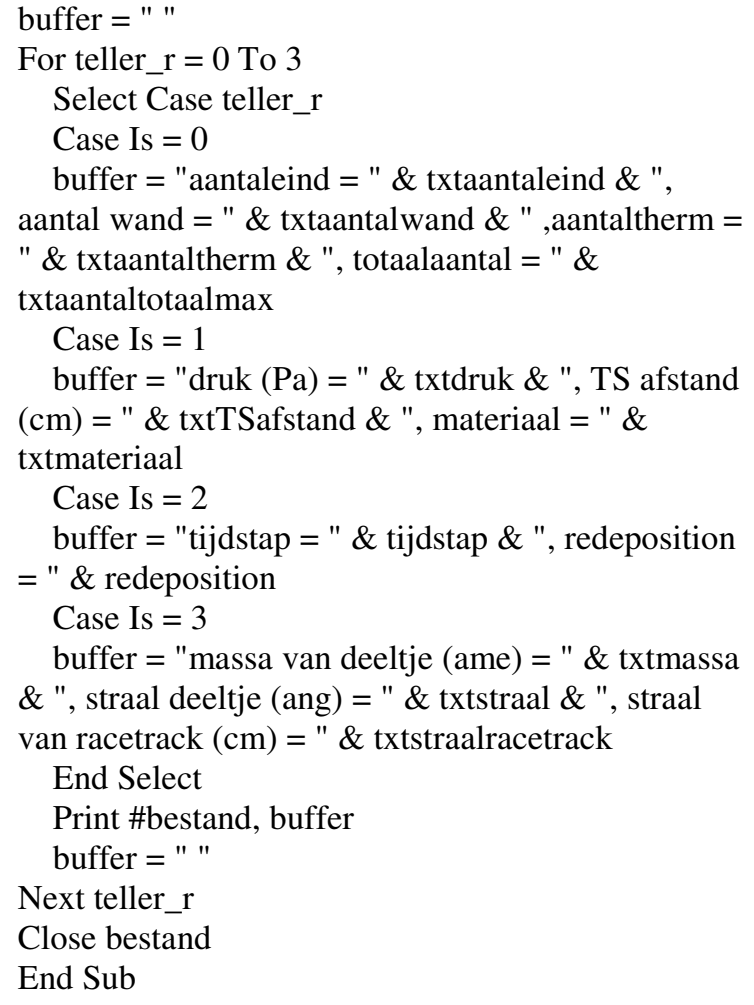

Code Transport of thermalized particles: Option Explicit

Public N As Double

Public bestand As Single

Public buffer As String

Public Ndeeltjes As Single

Public aantaleind As Single

Public aantalwand As Single

Public teller_r As Double

Public teller_k As Double

Public x As Double

Public y As Double

Public z As Double

Public theta As Double

Public phi As Double

Public halt As Integer

Public afstand As Single

Public landa As Double

Public P As Single

Public A As Single

Public B As Double

Public druk As Single

Public massa As Single

Public straal As Single

Public bmax As Single

Public teller As Single

Private Const conpi $=3.14159265358979$

Private matrixtherm $(1000000,6)$ As Single

Private matrixeind $(1000000,6)$ As Single

Private matrixwand(1000000, 6) As Single

Private Sub Command1_Click()

Randomize

$\mathrm{N}=$ Val(txtaantaldeeltjes)

'read file where thermalized particles were stored bestand $=$ FreeFile

buffer = "D:\mijn documenten $\backslash$ Stijn $\backslash$ sim richting

atomen $\mid \sin \backslash T i \_24 d e c \_0.68 \_13$ _therm.csv"

Open buffer For Input As bestand

For teller_r $=0$ To $(\mathrm{N}-1)$

For teller_k $=0$ To 6

Input \#bestand, matrixtherm(teller_r, teller_k)

Next teller_k

Input \#bestand, buffer

Next teller_r

Close bestand

'write initializing values in textbox

gegevens. Text $=$ txtdruk \& $" \mathrm{~Pa} / " \&$ txtmassa \& "

a.m.e./" \& txtafstand \& " cm/" \& txtaantaldeeltjes \& " aantal deeltjes"

'determine energy where particles are thermalized

'set counter on zero

aantalwand $=0$

aantaleind $=0$

Ndeeltjes $=-1$

druk $=$ Val(txtdruk)

massa $=$ Val(txtmassa)

straal $=$ Val $($ txtstraal $)$

'bmax $=5 *($ straal +0.0501$) * 110 / 20$

'landa $=13.178 /($ bmax $*$ bmax $*$ druk $)$

landa $=0.13178 /\left(\operatorname{druk} *((1+(\operatorname{massa} / 39.948)))^{\wedge}\right.$

$\left.0.5 *\left((\text { straal }+0.102)^{\wedge} 2\right)\right)$

afstand $=$ landa $/ 50$

$\mathrm{P}=1-\operatorname{Exp}(-1 / 50)$

'let run until number of particles is aantaltotaalmax

While Ndeeltjes < $(\mathrm{N}-1)$

Ndeeltjes $=$ Ndeeltjes +1

$\mathrm{x}=$ matrixtherm(Ndeeltjes, 0$)$

$\mathrm{y}=$ matrixtherm(Ndeeltjes, 1$)$

$\mathrm{z}=$ matrixtherm(Ndeeltjes, 2)

theta $=$ matrixtherm $($ Ndeeltjes, 3$)$

phi $=$ matrixtherm $($ Ndeeltjes, 4$)$

halt $=0$

Do While halt $=0$

Randomize

$\mathrm{A}=\mathrm{Rnd}^{\wedge} 1.05$

Select Case P

Case Is $>$ A

Randomize

'Do

theta $=$ Rnd $*$ conpi

'B = Rnd ^ ${ }^{\wedge} 1.05$

'Loop Until theta $>$ Atn(B / Sqr(-B * B +

1))

$$
\begin{aligned}
& \text { phi }=\text { Rnd } * 2 * \text { conpi } \\
& \mathrm{z}=\mathrm{z}+\text { afstand } * \text { Cos (theta) } \\
& \mathrm{x}=\mathrm{x}+\text { afstand } * \operatorname{Sin}(\text { theta }) * \operatorname{Cos}(\text { phi }) \\
& \mathrm{y}=\mathrm{y}+\text { afstand } * \operatorname{Sin}(\text { theta }) * \operatorname{Sin}(\text { phi }) \\
& \text { Case Is }<=\mathrm{A} \\
& \mathrm{z}=\mathrm{z}+\text { afstand } * \operatorname{Cos}(\text { theta }) \\
& \mathrm{x}=\mathrm{x}+\text { afstand } * \operatorname{Sin}(\text { theta }) * \operatorname{Cos}(\mathrm{phi}) \\
& \mathrm{y}=\mathrm{y}+\text { afstand } * \operatorname{Sin}(\text { theta }) * \operatorname{Sin}(\mathrm{phi})
\end{aligned}
$$

End Select

'look if particle is at substrate

If $\mathrm{z}>=\mathrm{Val}$ (txtafstand) Then 
matrixeind $($ aantaleind, 0$)=x$

matrixeind (aantaleind, 1$)=y$

matrixeind(aantaleind, 2$)=\mathrm{z}$

matrixeind (aantaleind, 3$)=$ theta

matrixeind (aantaleind, 4$)=$ phi

matrixeind (aantaleind, 5) $=0$

matrixeind (aantaleind, 6$)=0$

aantaleind $=$ aantaleind +1

halt $=1$

'look if particle is at walls

ElseIf $\mathrm{z}<0$ Or $\operatorname{Abs}(\mathrm{x})>25$ Or $\operatorname{Abs}(\mathrm{y})>$

25

Then

matrixwand (aantalwand, 0$)=x$

matrixwand (aantalwand, 1$)=y$

matrixwand(aantalwand, 2$)=\mathrm{z}$

matrixwand(aantalwand, 3 ) $=$ theta

matrixwand (aantalwand, 4) $=$ phi

matrixwand(aantaleind, 5) $=0$

matrixwand(aantaleind, 6$)=0$

aantalwand $=$ aantalwand +1

halt $=1$

End If

Loop

'this was end of loop because particle is at wall

\section{Wend}

'this is en of kloop because number of particles exeeded aantatotaalmax

bestand $=$ FreeFile

Open txtmateriaal \& "_" \& txtnaam \& "_" \& txtdruk \& "_" \& txtafstand \& "_" \& "thermeind" \& ".csv"

For Output As bestand

buffer $=$ " "

For teller_r $=0$ To (aantaleind -1$)$

For teller_k $=0$ To 6

buffer $=$ buffer $\&$ matrixeind(teller_r, teller_k) \& ","

Next teller_k

Print \#bestand, buffer buffer $="$ "

Next teller_r

Close bestand

bestand $=$ FreeFile

Open txtmateriaal \& "_" \& txtnaam \& "_" \& txtdruk \&

"_" \& txtafstand \& "_" \& "thermwand" \& ".csv" For

Output As bestand

buffer $="$ "

For teller_r $=0$ To (aantalwand - 1)

For teller_k $=0$ To 6 buffer $=$ buffer $\&$ matrixwand(teller_r, teller_k) \& ","

Next teller_k

Print \#bestand, buffer

buffer $=$ " "

Next teller_r

Close bestand

bestand $=$ FreeFile

Open txtmateriaal \& "_" \& txtnaam \& "_" \& txtdruk \&

"_" \& txtafstand \& "_" \& "thermplaats" \& ".txt" For

Output As bestand

buffer = " "

For teller_r $=0$ To 2

Select Case teller_r

Case Is $=0$

buffer $=$ "aantaleind $=$ " \& aantaleind $\&$ ", aantal

wand $=$ " \& aantalwand

Case Is $=1$

buffer $=$ "druk $(\mathrm{Pa})=$ " \& txtdruk \& ", TS afstand

$(\mathrm{cm})=" \&$ txtafstand \& $"$, materiaal $="$ \& txtmateriaal

Case Is $=2$

buffer $=$ "massa van deeltje (ame) $=$ " \& txtmassa $\&$

", straal deeltje $($ ang $)=" \&$ txtstraal

End Select

Print \#bestand, buffer

buffer $=$ " "

Next teller_r

Close bestand

End Sub 


\section{Chapter VIII: Other materials.}

\section{1: Introduction}

The mechanism of biaxial alignment in TiN and YSZ films deposited by reactive magnetron sputtering on a tilted substrate has been discussed in the previous chapters. Therefore, first the development of the out-of-plane alignment (chapter V), then the development of the inplane alignment (chapter VI) and finally the influence of several deposition parameters on the degree of in-plane alignment (chapter VII) has been discussed.

Although the proposed models were developed for TiN and YSZ, they should also be applicable for other materials. Therefore, the deposition technique as described in chapter III has been used to deposit other materials. For some of these materials $(\mathrm{Cr}, \mathrm{MgO}$ and Indium Tin Oxide) a biaxial alignment could be obtained, while for others ( $\mathrm{Al}, \mathrm{Cu}$ and $\mathrm{Ag}$ ) not. The results of the depositions of these materials and the correlation with the presented models will be briefly discussed in this chapter.

\section{2: Correlation between presented models and experimental observations for other materials}

\section{2.1: Indium Tin Oxide, ITO}

$\mathrm{In}_{2} \mathrm{O}_{3}$ has a C-rare earth structure [1]. The unit cell of such a C-rare earth structure is a combination of eight oxygen deficient unit cells of a fluorite structure in an appropriate manner. Doping such a C-rare earth $\operatorname{In}_{2} \mathrm{O}_{3}$ with Sn causes the replacement of some In atoms by $\mathrm{Sn}$ atoms and some additional $\mathrm{O}$ vacancies, but with retaining the $\mathrm{C}$-rare earth structure. Here, Indium Tin Oxide (ITO) films are deposited by sputtering a suboxide $\left(\operatorname{In}_{2} \mathrm{O}_{3}\right):\left(\mathrm{SnO}_{2}\right)$ 90:10\% target in an $\mathrm{Ar} / \mathrm{O}_{2}$ mixture.

As discussed in chapter V, also the deposited YSZ films have a fluorite structure in which some $\mathrm{Zr}$ atoms are replaced by $\mathrm{Y}$ atoms and with some $\mathrm{O}$ vacancies. For the calculation of the number of nearest neighbours offered by the low-index planes, a perfect fluorite structure was assumed (see table V.6.6).

Neglecting in a first approximation again the oxygen vacancies in the structure of the ITO films, the calculated number of nearest neighbours offered between the incoming adparticles and the growing low-index planes should be the same for YSZ and ITO since both materials consist of a fluorite-like structure.

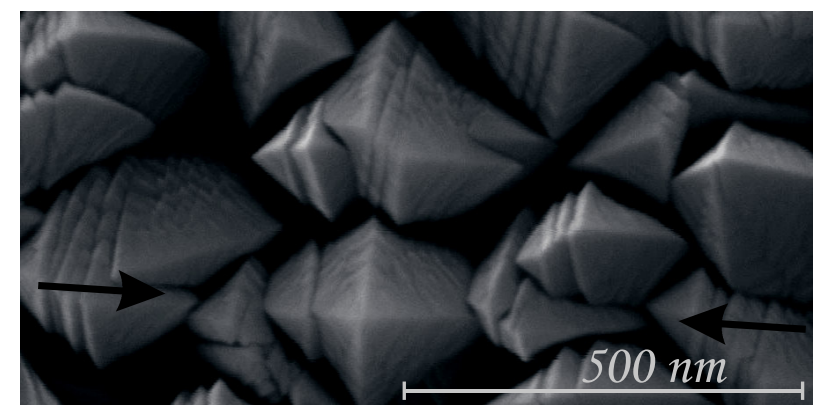

Figure VIII.2.1: SEM plan view of an ITO film deposited on grounded Hastelloy, at a target-substrate angle of $55^{\circ}$ and a target-substrate distance of $13 \mathrm{~cm}$. Well faceted, rectangular shaped column tops are observed. All grains align with a corner or ridge towards the orthogonal projection of the material flux, indicated by the black arrows.

Figure VIII.2.1 shows a SEM plan view of an ITO film deposited on a Hastelloy substrate. The substrate was $55^{\circ}$ tilted with respect to the material flux. The orthogonal projection of the incoming material flux is indicated by the black arrow. Well faceted columns are observed. It 
is also observed that these grains have a rectangular shape, as seen in plan view. These rectangular shaped grains all point with a corner towards the orthogonal projection of the material flux. Comparing these results, with the SEM results of an YSZ film deposited at zone T conditions (see fig. VI.2.9) indicate that the same microstructure is observed.

The ITO film has a nearly perfect [100] out-of-plane alignment, since the [400] peak is by far the most intensive one in the $\theta / 2 \theta$ spectrum, as shown in figure VIII.2.2. That corresponds again with the observations of YSZ, because also the YSZ films deposited in zone T conditions have a clear [100] out-of-plane orientation.

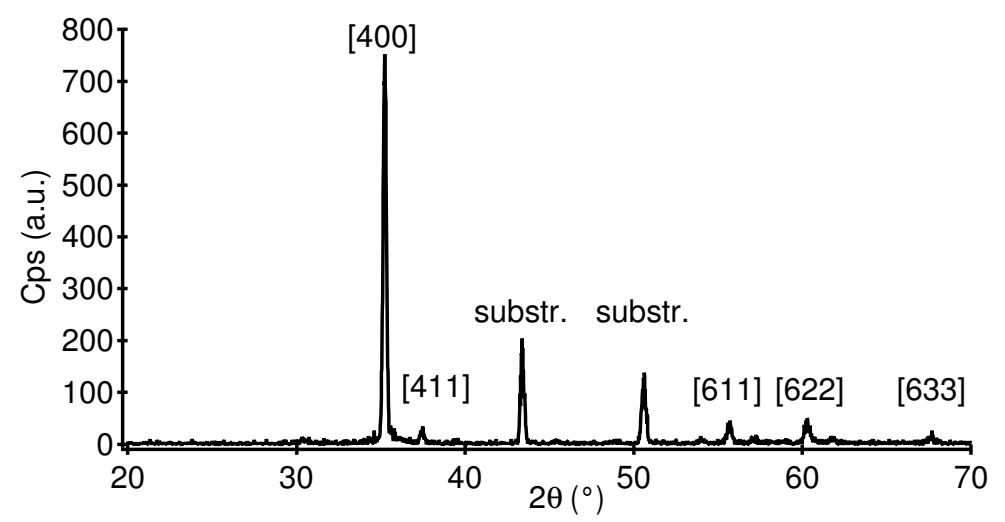

Figure VIII.2.2: XRD $\theta / 2 \theta$ spectrum of the same ITO film as in figure VIII.2.1. A clear [100] out-of-plane orientation is observed.

Finally, the in-plane alignment has been investigated by taking a $\{111\}$ pole figure, as shown in figure VIII.2.3. It is observed that the pole figure consists of 4 peaks, indicating the that film is in-plane oriented. The FWHM of the poles was $27.8^{\circ}$ in the phi direction. It is also observed that the poles are $45^{\circ}$ rotated in the phi-direction with respect to the orthogonal projection of the material flux, which is indicated by the black arrow. Again, this is in accordance with the YSZ observations, since also the $\{111\}$ pole figures of the YSZ films have four clear diffraction peaks, rotated by $45^{\circ}$ with respect to the orthogonal projection of the material flux (see fig. VI.2.8).

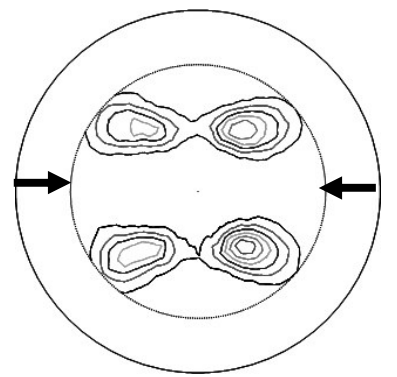

Figure VIII.2.3: A $\{111\}$ pole figure of the ITO film. The arrow indicates the orthogonal projection of the material flux. Four clear diffraction spots are observed, indicating a good in-plane alignment.

As discussed in chapter V, the [100] out-of-plane alignment and the $\{111\}$ crystal habit of the YSZ films deposited in zone T conditions was understood since the (100) plane has the highest geometric growth rate and the (111) plane the lowest, in the situation of metallic adatoms and molecular reactive gas (see table V.6.6). Although the plasma composition during the ITO deposition was not measured, it can be assumed that also for this deposition the metallic adparticles are adatoms and that the reactive gas is molecular. Since both materials have a fluorite-like structure, it is logic that both have the same out-of-plane alignment and the same crystal habit.

Due to the same crystal habit, it is also evident that both materials have the same in-plane alignment. 
It can be concluded that the proposed mechanism of biaxial alignment is also valid for indium tin oxide. Moreover, the proposed growth mechanism will be valid for all materials with a fluorite-like structure.

\section{2.2: Magnesium oxide, $\mathrm{MgO}$}

The growth of biaxially aligned $\mathrm{MgO}$ has been extensively investigated by my colleague Pieter Ghekiere. Since the results of these $\mathrm{MgO}$ depositions will be discussed in detail in his future thesis, only the basic results will be discussed here. More detailed results can also be found in some of his publications [2-4].

Just like TiN, $\mathrm{MgO}$ has a rocksalt structure. As such, since the proposed mechanism of biaxial alignment suggests the same growth for all materials of the same structure, $\mathrm{MgO}$ should give similar results as TiN.

Indeed, the biaxially aligned $\mathrm{MgO}$ thin films obtained by sputtering a $\mathrm{Mg}$ target in an $\mathrm{Ar} / \mathrm{O}_{2}$ mixture, have a [111] out-of-plane alignment and a zone T microstructure with $\{100\}$ faceted column tops. Also the observed in-plane alignment was similar to the in-plane alignment of TiN (see fig. VI.2.4) because the $\{100\}$ pole figures show three clear diffraction spots of which one pole points in the direction of the orthogonal projection of the material flux. Also the faceted column tops of $\mathrm{MgO}$ showed a clear microstructural in-plane alignment, again similar to the observed microstructure of biaxially aligned TiN films (see fig. VI.2.2).

Pieter Ghekiere reported also the influence of some deposition parameters on the degree of inplane alignment of these $\mathrm{MgO}$ thin films [2].

- An improving degree of in-plane alignment with increasing target-substrate angle was observed. The same influence of an increasing target-substrate angle on the degree of inplane alignment for TiN and YSZ films has been discussed in chapter VII (fig. VII.2.2). This improvement in in-plane alignment with increasing target-substrate angle was explained by a decreasing angular spread on the incoming material flux (fig. VII.6.1).

- Also an improvement in in-plane alignment with decreasing target-substrate distance was reported. The same influence was observed for the TiN depositions (see VII.2.4), which was again explained by a decreasing angular spread on the incoming material flux (fig. VII.6.2).

- An optimum in the in-plane alignment with decreasing pressure was observed for the $\mathrm{MgO}$ depositions, as well as for the TiN and YSZ depositions (fig. VII.2.3). That optimum was explained in fig. VII.6.3 by first a decreasing angular spread on the material flux with decreasing pressure (i.e. better in-plane alignment), followed by a too strong decrease of the mobility at too low pressures (i.e. worse in-plane alignment).

- Also an influence of the substrate bias on the degree of in-plane alignment of the $\mathrm{MgO}$ films was reported. As discussed in chapter VII (VII.6) this is due to a change in the mobility.

- Finally also the influence of an increasing film thickness on the in-plane alignment was investigated. First, an improving in-plane alignment with increasing film thickness was reported, similar to the observations for TiN and YSZ (fig.VII.2.5). This is in accordance with the suggested overgrowth mechanism.

However, Pieter Ghekiere also observed that the in-plane alignment get worse at too high film thicknesses. This was explained by an increasing surface temperature, and thus mobility, with increasing deposition time due to the long-time exposure of the growing film to the plasma-surface interaction. This increased mobility resulted in a zone II growth and thus in the disappearance of the in-plane alignment [4]. 
It can be concluded that the resulting out-of-plane and in-plane alignment of biaxially aligned $\mathrm{MgO}$ films are similar to these of TiN and are thus in accordance with the proposed growth mechanism. Also the influence of several deposition parameters was understood.

\section{2.3: Chromium, $\mathrm{Cr}$}

Also biaxially aligned $\mathrm{Cr}$ could be obtained by sputter deposition on a tilted substrate [5]. Since again all results are obtained by Pieter Ghekiere, and thus will be discussed in detail in his future thesis, only the basic results will be compared to the proposed mechanism of biaxial alignment.

Pieter observed that a biaxially aligned $\mathrm{Cr}$ film has a clear zone $\mathrm{T}$ structure, in which all the column tops are nicely faceted by $\{110\}$ planes. A clear preferential [100] out-of-plane alignment was observed. Taking a $\{110\}$ pole figure resulted in four diffraction spots, indicating an in-plane alignment. It was observed that the spots are also $45^{\circ}$ rotated in the phidirection with respect to the orthogonal projection of the material flux. SEM figures indicated that the grains are square shaped as seen in plan view, and also that all these square shaped grains point with a corner towards the orthogonal projection of the material flux.

$\mathrm{Cr}$ has a bcc structure. Therefore, the number of nearest neighbours offered between the incoming adparticles and the growing plane has to be calculated. Fortunately, there is only one situation, i.e. Cr adatoms, since no reactive gas is present during the deposition. Table VIII.2.4 gives that number of nearest neighbours for the three low-index planes. It is observed that the (110) plane offers the lowest number of nearest neighbours and thus will have the lowest crystallographic growth rate. This results in $\{110\}$ faceted grains. The growth according to this $\{110\}$ faceted crystal habit makes the [100] direction to have the largest geometric growth rate. As such, the observed $\{110\}$ crystal habit and the [100] out-of-plane alignment are in accordance with the proposed growth mechanism.

Table VIII.2.4: Number of nearest neighbours offered to a $\mathrm{Cr}$ adatom for the three low-index planes of a bcc structure.

\begin{tabular}{|l|l|l|c|}
\hline Low-index plane & $(100)$ & $(110)$ & $(111)$ \\
\hline $\begin{array}{l}\text { Number of nearest } \\
\text { neighbours }\end{array}$ & 4 & 2 & $\begin{array}{c}2.5 \\
(4 \text { or } 1)\end{array}$ \\
\hline
\end{tabular}

Also the observed microstructure and crystallographic in-plane alignment correspond to the proposed growth mechanism, since it was already calculated for YSZ that square shaped grains oriented with their corner towards the orthogonal projection of the material flux will overgrow all other grains (chapter VI, VI.3).

\section{2.4: Aluminium, copper and silver}

Thin films of $\mathrm{Al}, \mathrm{Cu}$ and $\mathrm{Ag}$ have been deposited by magnetron sputtering. However, although a large variation in target-substrate distance $(9-17 \mathrm{~cm})$, pressure $(0.55-1.7 \mathrm{~Pa})$, substrate temperature $(173 \mathrm{~K}-300 \mathrm{~K})$, discharge current $(0.5-1 \mathrm{~A})$ or film thickness $(0.8-8 \mu \mathrm{m})$ all films exhibit a more or less random out-of-plane orientation.

As described in chapter II, this random out-of-plane orientation of crystalline films indicates that the depositions were carried out in zone Ic or in zone III conditions. Figure VIII.2.5 shows a typical SEM plan view of the deposited fcc metal films. It is clearly observed that the grains (or columns) have a diameter of at least a few $\mu \mathrm{m}$, are not faceted and exhibit a rather blurred structure. Since the grains (or columns) also have a diameter which is much larger than their height, it can be concluded that the depositions were carried out in zone III conditions. 
It should be remarked that the obtained zone III structure is a possible result of the extreme sensitivity of pure metal films to impurities. Even a negligible amount of impurities can cause a zone III structure (see chapter II).

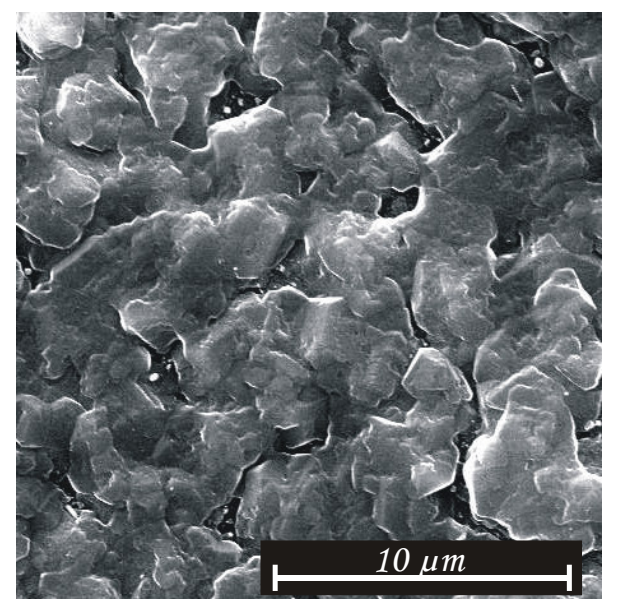

Figure VIII.2.5:Typical SEM plan view of a sputter deposited fcc metal film. Here an Al film, deposited at a target-substrate distance of $13 \mathrm{~cm}$, a pressure of $0.55 \mathrm{~Pa}$, discharge current of $0.9 \mathrm{~A}$ and on a non-tilted Ti foil substrate is shown.

Despite the possible influence of impurities, this growth in zone III conditions can be understood, because the barriers for surface or bulk diffusion in a fcc metal are much smaller than these for other classes of materials (bcc, rocksalt or fluorite structure). Typical values of these surface and bulk diffusion barriers are given in table VIII.2.6.

Table VIII.2.6: Typical values for the surface and bulk diffusion activation energies in fcc, bcc, rocksalt and fluorite structures [6].

\begin{tabular}{|l|l|l|l|l|}
\hline & fcc & bcc & rocksalt & fluorite \\
\hline Surface diffusion $(\mathrm{kJ} / \mathrm{mol})$ & $100-200$ & $300-580$ & $320-460$ & $450-625$ \\
\hline Buk diffusion $(\mathrm{kJ} / \mathrm{mol})$ & $60-170$ & $190-280$ & $200-250$ & $300-375$ \\
\hline
\end{tabular}

Due to these low activation energies for surface and bulk diffusion, the mobility on the growing film is much higher compared to other structures, allowing the re-structurative grain growth already at moderate temperatures. This was also already mentioned by Thompson et al. $[7,8]$, by comparing the critical homologous temperatures at which a transition between two zones occurs for several classes of materials.

It can be concluded that the experimental results of the deposited fcc metal films are not in contradiction with the proposed growth mechanisms, but neither provide additional evidence that these are right.

\section{3: Conclusions}

It is shown that the proposed models for the out-of-plane alignment and in-plane alignment are also able to explain the resulting biaxial alignment in $\mathrm{MgO}, \mathrm{Cr}$ and ITO films deposited with magnetron sputtering on a tilted substrate. Unfortunately, the validity of the proposed models for the fcc metals $\mathrm{Cu}, \mathrm{Al}$ and $\mathrm{Ag}$ could not be checked since no preferential orientation could be obtained in these films. 


\section{References:}

1: F.S. Galasso in "Structure and properties of inorganic solids" published by Pergamon press ISBN: 0080068731

2: P. Ghekiere, S. Mahieu, G. De Winter, R. De Gryse and D. Depla: J. Cryst. Growth 271 (2004) p.462-468

3: P. Ghekiere, S. Mahieu, G. De Winter, R. De Gryse and D. Depla: Thin Solid Films 493 (2005) p.129-134

4: P. Ghekiere, S. Mahieu, G. De Winter, R. De Gryse, D. Depla and O.I. Lebedev: Solid State Phenomena 105 (2005) p.433-438

5: P. Ghekiere, S. Mahieu, R. De Gryse and D. Depla "Structure evolution of the biaxial alignment in sputter-deposited $\mathrm{MgO}$ and Cr" accepted for publication in Thin Solid Films and presented at ICTF13, Stockholm, 2005

6: H.J. Frost and M.F. Ashby "Deformation Mechanism maps, the plasticity and creeps of metals and ceramics" at http://engineering.dartmouth.edu/ defmech/

7: C.V. Thompson: Ann. Rev. Mater. Sci. 30 (2000) p.159-190

8: C.V. Thompson and R. Carel: J. Mech. Phys. Solids 44(5) (1996) p.657-673 


\section{Chapter IX: Conclusions.}

Already in 1997, it was observed in the research group that reactive magnetron sputter deposition on a tilted random oriented polycrystalline or amorphous substrate could result in biaxially aligned thin films [1]. Such biaxially aligned thin films are polycrystalline films in which the grains not only share a crystallographic axis perpendicular to the substrate, but are also aligned along a reference direction parallel to the substrate.

In this thesis, the mechanism responsible for the development of that biaxial alignment has been described.

\section{Microstructure:}

Before discussing the crystallographic alignment, the thin film growth in general was discussed. This thin film growth could be seen as a sequence of the substeps: adsorption, diffusion and desorption, nucleation, crystal growth, re-structurative grain growth and further film growth.

The resulting microstructure of a thin film has been discussed as a function of the elementary process of diffusion and the fundamental phenomena of structure formation, i.e. nucleation, crystal growth and re-structurative grain growth. Depending on the occurrence of these phenomena of structure formation, the resulting structure could be categorized in one of the zones of the proposed structure zone model (SZM) as shown in table II.5.

Discussing that SZM, it was concluded that the films deposited at the conditions of only two zones, i.e. zone $\mathrm{T}$ and II, exhibit a preferential crystallographic out-of-plane alignment related to the deposition process.

At zone T conditions, the mobility during the deposition is high enough to complete the crystal growth, but too low to allow re-structurative grain growth by ripening, cluster migration or grain boundary diffusion. Therefore, the thin film growth is kinetically limited as the film is not able to reach its thermodynamic most favourable situation. Since diffusing adparticles are able to diffuse from one grain to another, neighbouring grains will compete with each other. That will result in a competitive growth, in which the grains oriented with the geometric fastest growing direction perpendicular to the substrate will overgrow all other grains. This results in a columnar structure, in which the columns are all nicely faceted and are all oriented with the fastest growing direction perpendicular to the substrate.

At zone II conditions, the mobility is high enough to allow re-structurative grain growth causing the growth to be thermodynamically driven. At these conditions, the total film is able to aim for its thermodynamic most stable situation and thus to aim for the lowest surface energy. This results in a columnar structure in which all the columns are oriented with the crystallographic plane of lowest surface energy parallel to the substrate.

\section{Out-of-plane alignment:}

It could be concluded that one should know the geometric fastest growing direction and the plane of lowest surface energy to completely understand the resulting preferential out-ofplane orientation in zone $\mathrm{T}$ and zone II respectively.

That plane of lowest surface energy is easily found in literature for most materials. However, the anisotropy in growth rate of the several crystallographic planes had to be discussed in more detail.

It was argued that the crystallographic growth rate of a crystallographic plane depends on the sticking coefficient and on the mobility of the adparticles on that plane. Both parameters were shown to depend on the number of nearest neighbours offered between the incoming 
adparticle and the growing plane. For both parameters, the crystallographic growth rate was reasoned to increase with increasing number of offered nearest neighbours.

Of course, that number of nearest neighbours offered between the incoming adparticle and the growing plane depends on the state of the adparticles and on the termination of the growing plane. Since the termination of the growing plane mainly depends on the state of the reactive gas (atomic or molecular), that number of nearest neighbours had to be calculated in the situation of metallic adatoms or metallic admolecules, and in the situation of atomic or molecular reactive gas.

Finally, it was argued that the crystallographic plane offering the lowest number of nearest neighbours will form the resulting facets of the specific crystal habit. This growth according to the kinetically determined crystal habit causes an anisotropy in geometric growth rate. The grains oriented with the geometric fastest growing direction perpendicular to the substrate will overgrow all other grains.

That proposed growth mechanism has been discussed in detail for TiN and YSZ, but could also be applied to understand the resulting microstructure and preferential out-of-plane orientation of ITO, MgO [2-4] and $\mathrm{Cr}$ [5] films. Carrying out mass-spectroscopy to know the plasma composition showed that all experimental results were in accordance with the proposed growth mechanism.

\section{In-plane alignment:}

Finally, also the development of the in-plane alignment when carrying out the deposition on a tilted substrate was discussed.

Since it was observed that all biaxially aligned thin films were deposited at zone T conditions, the film growth in zone $\mathrm{T}$ had to be examined in more detail.

It was discussed that carrying out the deposition in zone $\mathrm{T}$, causes the grains to expand by $2 \mathrm{D}$ and $3 \mathrm{D}$ growth. 3D growth was described as the expansion of the grains due to the incorporation of adparticles directly from the gas phase, while 2D growth was described as the expansion due to the capture of adparticles which first hitted the bare substrate or another grain and then by diffusion get incorporated in the expanding grain.

By carrying out specific two-stepped depositions, it could be shown that the in-plane alignment is mainly caused by the $2 \mathrm{D}$ growth.

Then a model, explaining the in-plane alignment for depositions in zone $\mathrm{T}$ conditions on a tilted substrate, assuming a 2D growth was proposed.

Carrying out the deposition on a tilted substrate causes the diffusion of the adparticles to be directed due to the conservation of their momentum along the orthogonal projection of the incoming direction. Assuming in a first approximation that all adparticles diffuse along one single direction, the amount of adparticles captured by a faceted grain as a function of its inplane orientation could be calculated. This calculation indicated that grains oriented along a specific in-plane orientation will capture more diffusing adparticles, thus will grow faster and consequently will be able to overgrow all other grains, causing the in-plane alignment.

In case of square shaped grains, as seen in plan view, it was calculated that the grains pointing with a corner towards the orthogonal projection of the incoming material flux will expand the fastest and thus will overgrow all other grains. This result was in accordance with the experimental observations of YSZ, ITO and Cr [5].

However, in case of triangular shaped grains as seen in plan view ( $\mathrm{TiN}$ and $\mathrm{MgO}$ [2-4]), it was calculated that the grains with a corner towards the orthogonal projection of the incoming material flux should have the same capture length and thus the same growth rate as the grains 
oriented with an edge towards the incoming material flux. Although a suggestion is given, further research should be carried out to know why the grains oriented with an edge towards the orthogonal projection of the incoming material flux survive instead of the grains with a corner towards the incoming material flux.

Beside the development of the in-plane alignment, it was also observed that the degree of inplane alignment, which is expressed by the FWHM of the diffraction peak in a pole figure, is strongly influenced by several deposition parameters.

It was shown that the degree of in-plane alignment is influenced by two fundamental parameters: the mobility during the growth and the angular spread on the incoming material flux.

The mobility during the growth influences the degree of in-plane alignment, since the in-plane alignment improves, the more the deposition was carried out in zone T conditions. Decreasing the mobility too much, results in an incomplete $2 \mathrm{D}$ growth and thus in a worse in-plane alignment.

An angular spread on the incoming material flux was shown to cause the same angular spread on the "right" in-plane orientation. Calculating the angular spread on the incoming material flux, by a binary collision Monte Carlo simulation program, provided a quantitative value for the best obtainable in-plane alignment.

It was shown that the influence of the target-substrate distance, target-substrate angle, pressure, conditions of the chamber walls, substrate bias, $\mathrm{Ar} / \mathrm{O}_{2}$ ratio, target usage and magnet configuration on the degree of in-plane alignment of the YSZ or TiN films could be understood as a variation of the mobility or of the angular spread on the incoming material flux.

Finally, it can be concluded that the development of the biaxial alignment in thin films deposited by reactive magnetron sputtering on a tilted substrate, is understood if one:

- calculates the energy flux towards the substrate, to know the mobility at the growing films and thus the zone at which the deposition was carried out.

- measures the plasma composition by energy resolved mass spectroscopy to know in which situation (metallic adatoms or admolecules and atomic or molecular reactive gas) of zone $\mathrm{T}$ the deposition was carried out.

- simulates the collisional transport of the sputtered particles towards the substrate to know the angular spread on the incoming material flux, and thus to know an optimal degree of in-plane alignment.

Further research to the energy flux towards the substrate as a function of the used deposition conditions should be carried out, to have a quantitative value related to the adparticle mobility. 


\section{References:}

1: J. Denul: Ph.D thesis: "Sputterdepositie van $\mathrm{ZrO}_{2}: \mathrm{Y}_{2} \mathrm{O}_{3}$ bufferlagen en $\mathrm{YBa}_{2} \mathrm{Cu}_{3} \mathrm{O}_{7-\mathrm{x}}$ toplagen: invloed van de depositieparameters op de groei en de eigenschappen" UGent 1997 1998.

2: P. Ghekiere, S. Mahieu, G. De Winter, R. De Gryse and D. Depla: J. Cryst. Growth 271 (2004) p.462-468

3: P. Ghekiere, S. Mahieu, G. De Winter, R. De Gryse and D. Depla: Thin Solid Films 493 (2005) p.129-134

4: P. Ghekiere, S. Mahieu, G. De Winter, R. De Gryse, D. Depla and O.I. Lebedev: Solid State Phenomena 105 (2005) p.433-438

5: P. Ghekiere, S. Mahieu, R. De Gryse and D. Depla "Structure evolution of the biaxial alignment in sputter-deposited $\mathrm{MgO}$ and Cr" accepted for publication in Thin Solid Films and presented at ICTF13, Stockholm, 2005 


\section{Publication List:}

A1 Publications:

1: G. De Winter, S. Mahieu, I. De Roeck, R. De Gryse, J. Denul: IEEE T. Appl. Supercond. 13(2) (2003) p.2567-2570 "Unbalanced magnetron sputtering of biaxially aligned Yttria Stabilized Zirconia and Indium Tin Oxide"

2: S. Mahieu, G. De Winter, D. Depla, R. De Gryse and J. Denul: Surf. Coat. Technol. 187 (2004) p.122-130 "A model for the development of biaxial alignment in yttria stabilized zirconia layers, deposited by unbalanced magnetron sputtering."

3: P. Ghekiere, S. Mahieu, G. De Winter, R. De Gryse, D. Depla: J. Cryst. Growth 271 (2004) p.462-468 "Influence of the deposition parameters on the biaxial alignment of $\mathrm{MgO}$ grown by unbalanced magnetron sputtering."

4: S. Mahieu, P. Ghekiere, G. De Winter, S. Heirwegh, D. Depla and R. De Gryse O.I. Lebedev and G. Van Tendeloo: J. Cryst. Growth. 279(2005) p.100-109

"Mechanism of preferential orientation in sputter deposited titanium nitride and yttria stabilized zirconia layers. "

5: S. Mahieu, P. Ghekiere, G. De Winter, D. Depla, R. De Gryse, O.I. Lebedev and G. Van Tendeloo: Thin Solid Films 484 (2005) p.18-25 "Influence of the $\mathrm{Ar} / \mathrm{O}_{2}$ ratio on the growth and biaxial alignment of yttria stabilized zirconia layers during reactive unbalanced magnetron sputtering."

6: S. Mahieu, P. Ghekiere, G. De Winter, R. De Gryse, D. Depla, O. I. Lebedev and G. Van Tendeloo: Solid State Phenomena 105 (2005) p.447-452 "Biaxially aligned Yttria Stabilized Zirconia and Titanium Nitride layers deposited by unbalanced magnetron sputtering."

7: P. Ghekiere, S. Mahieu, G. De Winter, R. De Gryse, D. Depla: Thin Solid Films 493 (2005) p.129-134 "Scanning electron microscopy study of the growth mechanism of biaxially aligned magnesium oxide layers grown by unbalanced magnetron sputtering."

8: P. Ghekiere, S. Mahieu, G. De Winter, R. De Gryse, D. Depla and O.I. Lebedev: Solid State Phenomena 105 (2005) p.433-438 "Growth mechanism of biaxially aligned $\mathrm{MgO}$ deposited by unbalanced magnetron sputtering."

9: S. Mahieu, P. Ghekiere, G. De Winter, R. De Gryse, D. Depla, G. Van Tendeloo and O.I. Lebedev: Surf. Coat. Technol. 200 (8) (2006) p.2764-2768 "Biaxially aligned Titanium Nitride thin films deposited by reactive unbalanced magnetron sputtering." 10: S. Mahieu, G. Buyle, D. Depla, S. Heirwegh, P. Ghekiere and R. De Gryse, Nucl. Instr. Meth. B 243 (2) (2006) p.313-319 “ Monte Carlo simulation of the transport of atoms in DC magnetron sputtering."

11: S. Mahieu, P. Ghekiere, D. Depla, R. De Gryse, O.I. Lebedev and G. Van Tendeloo: J. Cryst. Growth 290 (2006) p.272-279 "Mechanism of in-plane alignment in magnetron sputtered biaxially aligned yttria-stabilized zirconia."

12: S. Mahieu, G. Buyle, P. Ghekiere, S. Heirwegh, R. De Gryse and D. Depla: accepted for publication in Thin Solid Films "Mechanism of biaxial alignment in thin films, deposited by magnetron sputtering."

13: P. Ghekiere, S. Mahieu, R. De Gryse and D. Depla: accepted for publication in Thin Solid Films "Structure evolution of the biaxial alignment in sputter deposited Mgo and Cr".

14: Review article in Thin Solid Films S. Mahieu, P. Ghekiere, D. Depla and R. De Gryse: "Biaxial alignment in sputter deposited thin films" submitted in April 2006. 
Other publications with reader committee:

1: S. Mahieu, G. De Winter, R. De Gryse, J. Denul: Institute of Physics Conference Series Number 181 (2004) ISBN 0-7503-0981 4 p.1469-1474 "Deposition of biaxially aligned YSZ layers by dual unbalanced magnetron sputtering"

Publications in journals without a reader committee:

1: S. Mahieu, G. De Winter, P. Ghekiere, D. Depla, R. De Gryse, J. Denul: Belvac 20(1) (2004) p.6-16 "Biaxially aligned YSZ layers deposited by unbalanced magnetron sputtering: a model."

2: S. Mahieu, G. Buyle, D. Depla, S. Heirwegh, P. Ghekiere and R. De Gryse: Belvac 21 (2) (2005) p.11-20 "Transport of DC sputtered atoms: a Monte Carlo simulation." 3: P. Ghekiere, S. Mahieu, G. De Winter, R. De Gryse, D. Depla: Belvac 21 (1) (2005) P.5-16 "Growth and optimization of biaxially aligned $\mathrm{MgO}$ deposited by unbalanced magnetron sputtering."

Abstracts of lectures on international conferences or symposia:

1: S. Mahieu, P. Ghekiere, G. De Winter, O.I. Lebedev, D. Depla, R. De Gryse:

"Mechanism of preferential orientation in thin films deposited by unbalanced magnetron sputtering." "Reactive Sputter processes and related Phenomena III" 9$10 / 12 / 2004$ in Ghent

2: S. Mahieu, P. Ghekiere, S. Heirwegh, R. De Gryse and D. Depla: "Mechanism of biaxial alignment in thin films, deposited by magnetron sputtering." "13th International Conference on Thin films: ICTF13" 20-23/06/2005 in Stockholm (Sweden)

3: P. Ghekiere, S. Mahieu, R. De Gryse and D. Depla: "Structure evolution of the biaxial alignment in sputter deposited $\mathrm{MgO}$ and Cr." "13th International Conference on Thin films: ICTF13" 20-23/06/2005 in Stockholm (Sweden)

4: invited S. Mahieu, P. Ghekiere, R. De Gryse and D. Depla: "Mechanism of biaxial alignment in thin films." "Reactive sputter deposition" 8-9/12/2004 in Delft (the Netherlands)

5: P. Ghekiere, S. Mahieu, D. Depla, R. De Gryse, O.I. Lebedev and G. Van Tendeloo: "Impurity induced texture change in chromium." "Reactive sputter deposition" 89/12/2005 in Delft (the Netherlands)

Abstracts of poster presentations on international conferences or symposia:

1: G. De Winter, S. Mahieu, I. De Roeck, R. De Gryse, J. Denul: Applied Superconductivity Conference 4-9/9/2002 Boston (United States) "Unbalanced magnetron sputter deposition of biaxially aligned YSZ and ITO films."

2: S. Mahieu, G. De Winter, R. De Gryse, J. Denul: 6th European Conference on Applied Superconductivity EUCAS 14-18/09/2003 in Sorrento (Italy) "Deposition of biaxially aligned YSZ by dual unbalanced magnetron sputtering."

3: S. Mahieu, G. De Winter, P. Ghekiere, R. De Gryse and D. Depla: 3th Nouvelles Tendances en Procédés Magnetron et Arc pour le depot de Couches Minces 2425/11/2003 "Investigation of the mechanism of biaxial alignment in thin films deposited by unbalanced magnetron sputtering."

4: S. Mahieu, P. Ghekiere, G. De Winter, R. De Gryse and D. Depla: International Vacuum Conference 28/06/2004-2/07/2004 in Venice (Italy) "Reactive sputter deposition of biaxially aligned TiN by unbalanced magnetron sputtering." 
5: P. Ghekiere, S. Mahieu, G. De Winter, R. De Gryse and D. Depla: International Vacuum Conference 28/06/2004-2/07/2004 in Venice (Italy) "Mechanism of biaxially aligned $\mathrm{MgO}$ grown by unbalanced magnetron sputtering."

6: S. Mahieu, P. Ghekiere, G. De Winter, R. De Gryse, D. Depla and O.I. Lebedev: $2^{\text {de }}$ International conference on Texture and Anisotropy of Polycrystals ITAP 7-9/07/2004 in Metz (France) " Biaxially aligned YSZ and TiN layers deposited by unbalanced magnetron sputtering."

7: P. Ghekiere, S. Mahieu, G. De Winter, O.I. Lebedev, R. De Gryse and D. Depla: $2^{\text {de }}$ International conference on Texture and Anisotropy of Polycrystals ITAP 7-9/07/2004 in Metz (France) "Growth mechanism of biaxially aligned $\mathrm{MgO}$ deposited by unbalanced magnetron sputtering."

8: S.Heirwegh, S.Mahieu, P.Ghekiere, D.Depla, R.De Gryse: Reactive Sputter Deposition 8-9/12/2005 in Delft (the Netherlands) "Reactive sputter deposition of aluminium oxide: influence of target erosion" 